Supplementary Materials for

\title{
Total Synthesis of Tagetitoxin
}

Chi He, Hang Chu, Thomas P. Stratton, David Kossler, Kelly J. Eberle, Dillon T. Flood, and Phil S. Baran*

Department of Chemistry, The Scripps Research Institute, 10550 North Torrey Pines Road, La Jolla, California, 92037, United States

Correspondence to: pbaran@scripps.edu

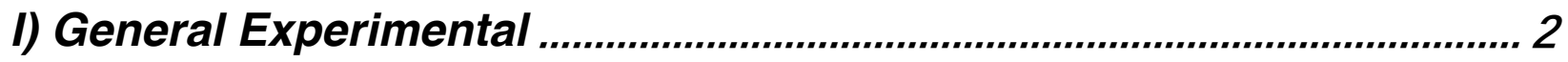

II) Summary of First-Generation Synthesis Optimization.......................... 3

III) Summary of Second-Generation Synthesis Optimization ................ 11

IV) Summary of Third-Generation Synthesis Optimization..................... 23

V) Experimental Procedures and Characterization Data ......................... 29

VI) Direct RNA Polymerase Inhibition Assay .......................................... 53

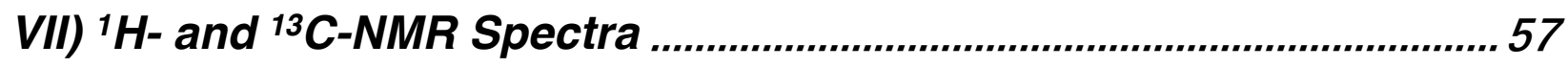

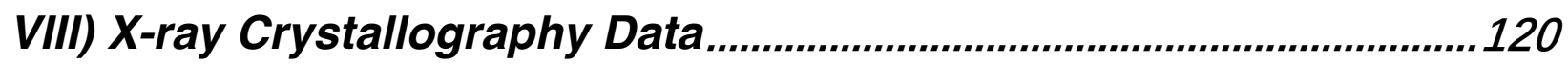

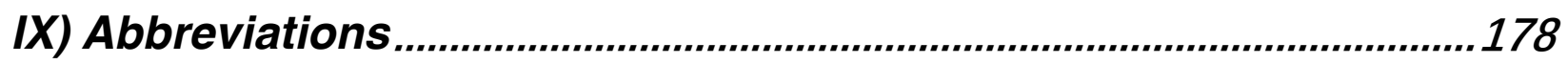

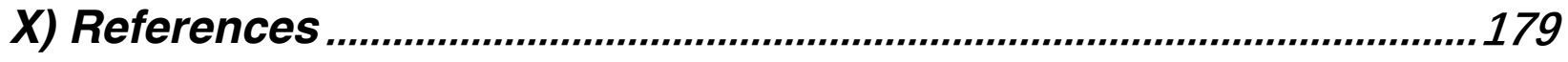




\section{I) General Experimental}

All reactions were carried out under an inert argon atmosphere with dry solvents under anhydrous conditions unless otherwise stated. Dry acetonitrile (MeCN), dichloromethane (DCM), diethyl ether ( $\left.\mathrm{Et}_{2} \mathrm{O}\right)$, tetrahydrofuran (THF), toluene (PhMe), dimethylformamide (DMF), benzene, and triethylamine $\left(\mathrm{Et}_{3} \mathrm{~N}\right)$ were obtained by passing the previously degassed solvents through activated alumina columns. Reagents were purchased at the highest commercial quality and used without further purification, unless otherwise stated. Yields refer to chromatographically and spectroscopically ( ${ }^{1} \mathrm{H}$ NMR) homogeneous material, unless otherwise stated. Reactions were monitored by thin layer chromatography (TLC) carried out on $0.25 \mathrm{~mm}$ E. Merck silica plates (60F254), using UV light as the visualizing agent and/or phosphomolybdic acid and heat as a developing agent. Flash silica gel chromatography was performed using E. Merck silica gel (60, particle size $0.043-0.063 \mathrm{~mm}$ ). NMR spectra were recorded on Bruker DRX-600 and AMX-400 instruments and were calibrated using residual undeuterated solvent as an internal reference (chloroform- $d$ : ${ }^{1} \mathrm{H}$ NMR $\delta=7.26 \mathrm{ppm},{ }^{13} \mathrm{C}$ NMR $\delta=77.16 \mathrm{ppm}$ ). The following abbreviations were used to explain NMR peak multiplicities: $s=$ singlet, $d=$ doublet, $t=$ triplet, $q=$ quartet, $m=$ multiplet, br = broad. High-resolution mass spectra (HRMS) were recorded on an Agilent LC/MSD TOF mass spectrometer by electrospray ionization time-of-flight (ESI-TOF) reflectron experiments. Melting points (M. P.) were recorded on an Electrothermal Mel-Temp digital melting point apparatus. 


\section{II) Summary of First-Generation Synthesis Optimization}

First-generation route: the construction of trans-[6,5]-bicycle

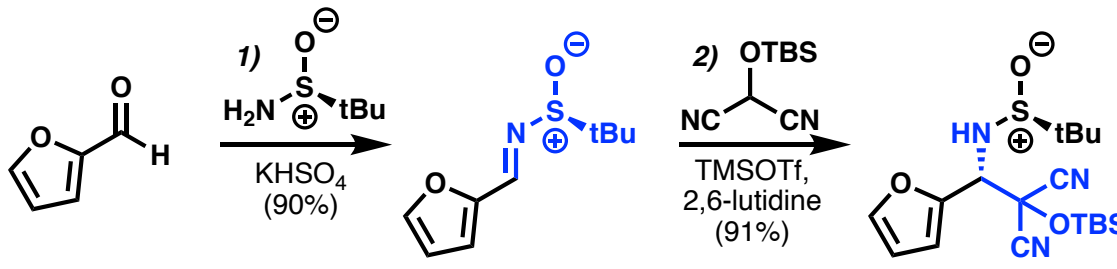<smiles>CCOC(=O)[C@H](N[Sb]([O-])(CC)OCC)c1ccco1</smiles>

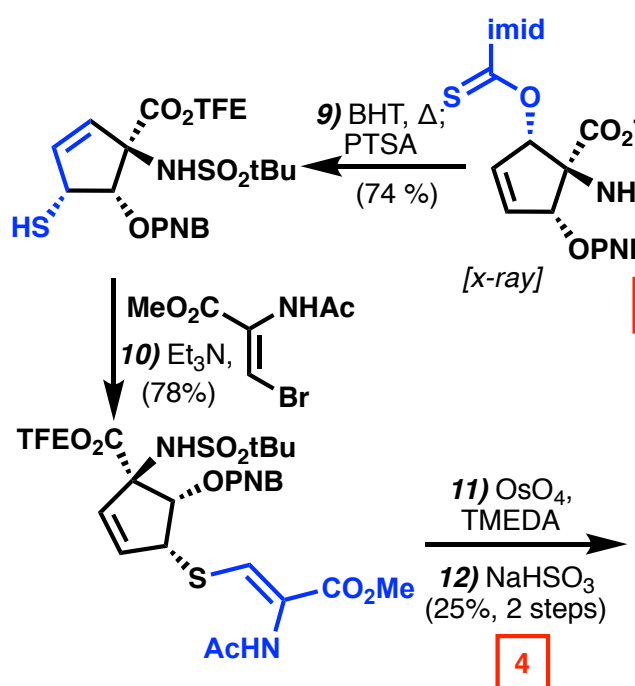

3

7) $\mathrm{NaBH}_{4}$, $\mathrm{CeCl}_{3} \cdot 7 \mathrm{H}_{2} \mathrm{O}$
$(50 \%, 5: 1$ d.r. $)$ (B) $\mathrm{ThioCDI}_{(88 \%)}$

$$
3 \text { [x-ray] }
$$
OPNB

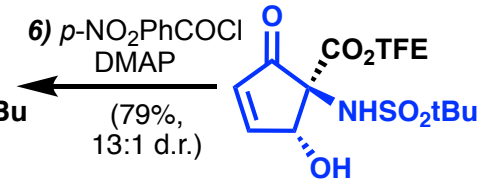
\begin{tabular}{|c|c|c} 
4) ${ }^{1} \mathrm{O}_{2} ;$ & $\begin{array}{c}\text { 5) } \mathrm{RuCl}_{3} \text { (cat.), } \\
\mathrm{NalO}_{4} \\
\mathrm{DMS}^{2}, \mathrm{SiO}_{2} \\
(55 \%, 1.2: 1 \text { d.r. })\end{array}$ \\
$(50 \% 8: 1$ d.r. $)$
\end{tabular}

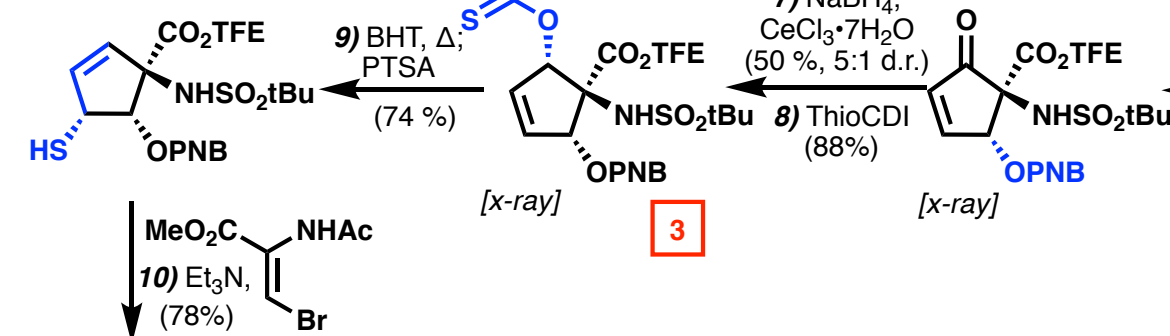

6) $p-\mathrm{NO}_{2} \mathrm{PhCOCl}$ 13:1 d.r.)

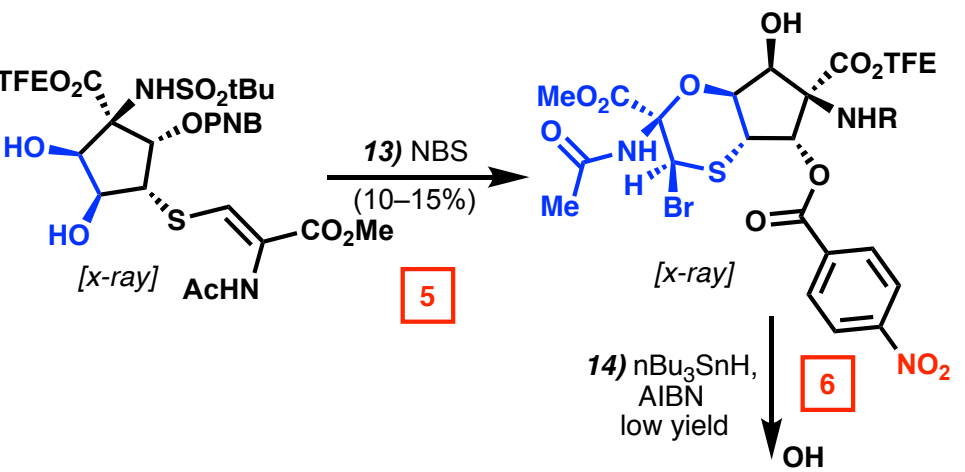

\section{Reaction Optimization}

1 - Amino-ester synthesis (p. SI-4)
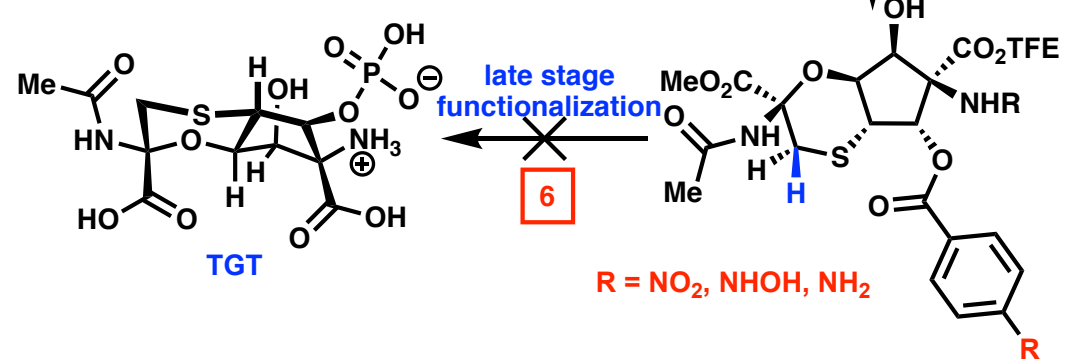

2 - Oxidative rearrangement (p. SI-5)

3 - Previous strategies towards fully-substituted cyclopentane (p. SI-7)

4 - Construction of side chain and dihydroxylation; full optimization in 2nd generation route (p. SI-13)

5 - Construction of trans-[6,5]-1,4-oxathiane, full optimization in 2nd generation route (p. SI-16)

6 - Debromination and other efforts (p. SI-9)

Key challenges:

2 - Erosion of ee in oxidative furan rearrangement (p. SI-6)

6 - FG incompatability (PNB group); other endgame efforts (p. SI-10)

Scheme S1. First-generation route to trans-[6,5]-bicycle 
Original conditions:<smiles>CC(C)(C)[Sb]([O-])/N=C/c1ccco1</smiles>

Optimization:

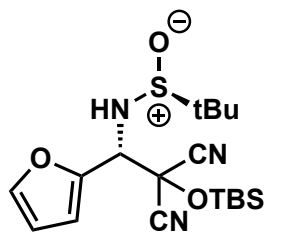

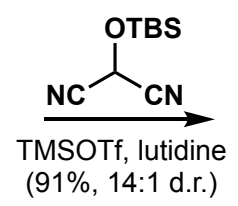

(91\%, 14:1 d.r.)<smiles>CC(C)(C)O[Sb]([O-])N[C@H](c1ccco1)C(C)(C#N)C(C)(C)C</smiles>

CN

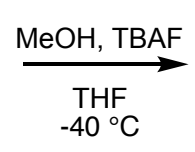<smiles>CCCCCCN[C@@H](C(=O)OC)c1ccco1</smiles>

(31\%)<smiles>CC(C)(C)[Sb]([O-])/N=C/c1ccco1</smiles>

(55\%)<smiles>CC(C)(C)OC(=O)[C@H](N[Sb]([O-])OC(C)(C)C)c1ccco1</smiles>

$R=$ TFE,$>60 \%$ yield

\begin{tabular}{|cc|}
\hline ROH: & Results: \\
TFE & Clean conversion, no sulfinimine \\
TCE & Partial conversion, messy, sulfinimine \\
BnOH & Partial conversion, messy \\
PMB-OH & Decaboxylation + PMB-OH \\
iPrOH & Slow, messy \\
tBuOH & Unstable product \\
\hline
\end{tabular}

Scheme S2. Amino-ester synthesis 
2 - Oxidative rearrangement

1) Optimization of reductant in rearrangement:

- Original condition:

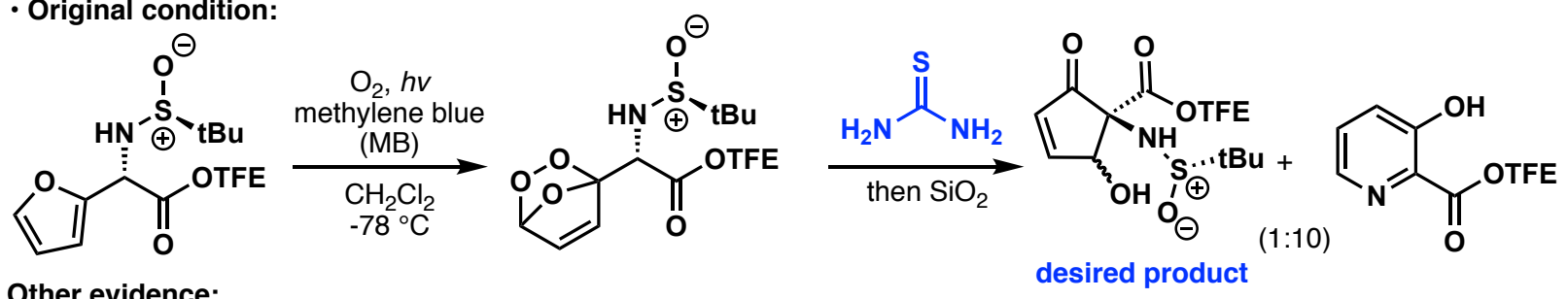

Other evidence: desired product

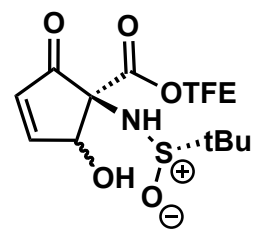

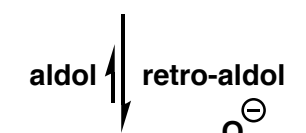<smiles>CC(C)(C)OC(=O)C(N[Si]([O-])C(C)(C)C)=C(O)/C=C\C=O</smiles>

- Modification:

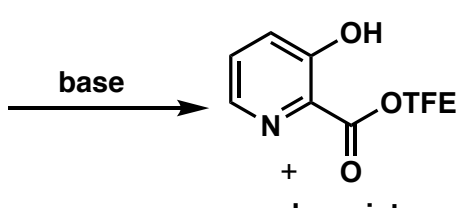
complex mixture<smiles>COC(=O)/C(N[Si](=O)OC(C)(C)C)=C(O)\C=C/C=O</smiles><smiles>CCOC(=O)c1ncccc1O</smiles>

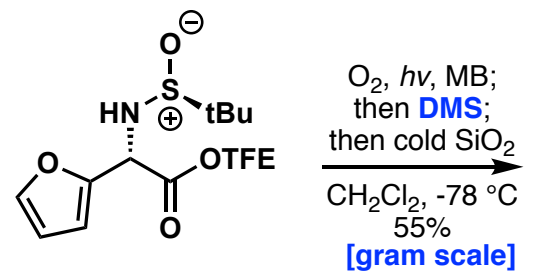<smiles>COC(=O)[C@@]1(N[Sb]([O-])(O)[C+](C)(C)C)C(=O)C=C[C@H]1O</smiles><smiles>[14CH3]</smiles>

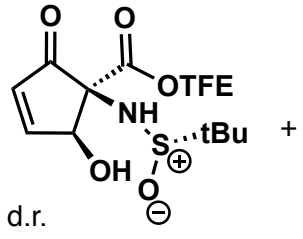<smiles>CC(C)OC(=O)c1ncccc1O</smiles>

Scheme S3. Optimization of reductant in oxidative rearrangement 
2) Details regarding erosion of ee in oxidative furan rearrangement:

- Early observation: Racemization occurred between oxidative rearrangement and alcohol acylation step

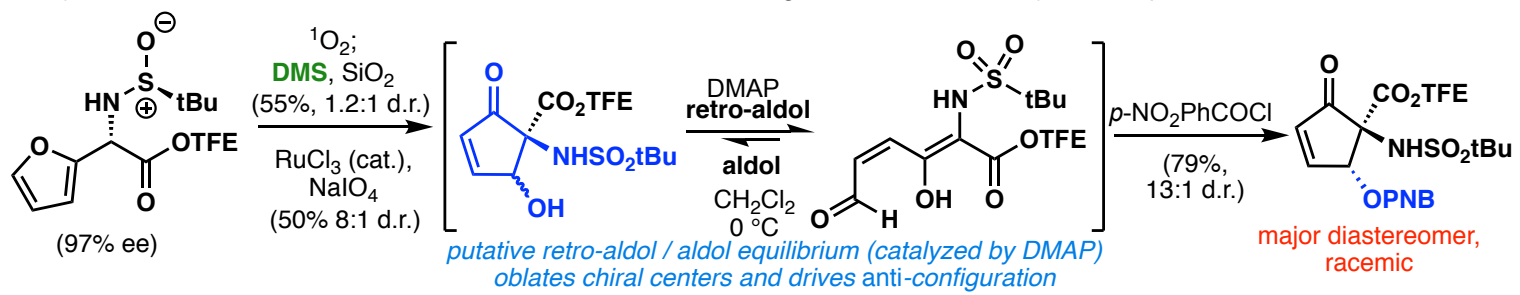

- Can acylation of alcohol immediately after oxidative rearrangement shut down retro-aldol and result in enantiopure product?<smiles>CC(C)(C)OC(=O)[C@H](N[SH](=O)[O-])c1ccco1</smiles>

$(97 \%$ ee) a) ${ }^{1} \mathrm{O}_{2}$; $\stackrel{\mathrm{DMS}, \mathrm{SiO}_{2}}{\longrightarrow}$ b) $\mathrm{PCNBCl}$ DMAP<smiles>C[C@H]1C=CC(=O)[C@]1(N[Si](=O)OC(C)(C)C)OC(F)F</smiles>
OPCNB

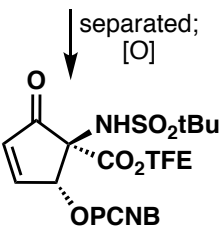

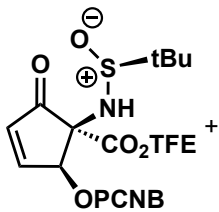

4 diastereomers (theoretical) (16.7:11:3.3:1 d.r.) separable, but difficult

- Furan SM is over $97 \%$ ee. - PCNB protection and oxidation steps were determined to not cause erosion of enantiopurity (confirmed by control experiments).

Erosion of enantiopurity observed $(70 \%$ ee)

Hypothesis: S-chiral center of Ellman chiral auxiliary partially racemized during photo ${ }^{1} \mathrm{O}_{2}$ step.<smiles>CCOC(=O)[C@H](N[SH]([O-])OCC)c1ccco1</smiles>

$(97 \%$ ee)

a) ${ }^{1} \mathrm{O}_{2}$, additive,

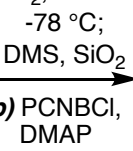

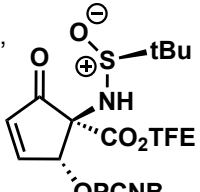
OPCNB

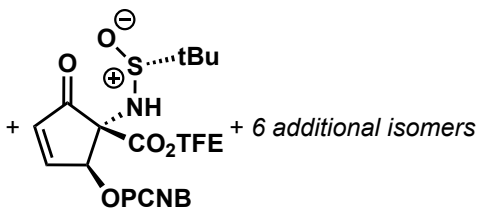

two major enantiomers

- Two major enantiomers (85:15); 70\% ee was observed.

- Significant improvement $(\mathbf{9 5 : 5}, \mathbf{9 0} \%$ ee) was observed with BHT (0.5 eq) as an additive in photo ${ }^{1} \mathrm{O}_{2}$ reaction. However, isolation of the 2 desired enantiomers from the resulting isomeric mixture (8 isomers) proved difficult.

- The two major enantiomers existed as a viscous oil and thus recrystallization was not a viable purification strategy (see picture below) - Alternate methods to generate ${ }^{1} \mathrm{O}_{2}$ (in absense of light) proved unsuccessful.

scale (50 mol\% BHT) ee

- $30 \mathrm{mg} \quad 90 \%$

. $700 \mathrm{mg} \quad 86 \%$

\begin{tabular}{l}
$\cdot 1.5 \mathrm{~g} \quad 83 \%$ \\
\hline
\end{tabular}

Dark ${ }^{1} \mathrm{O}_{2}$ methods Results

- $\mathrm{O}_{3}, \mathrm{Et}_{3} \mathrm{SiH}, 2$ equiv no reaction

- $\mathrm{H}_{2} \mathrm{O}_{2}, \mathrm{NaClO}$ no reaction

- $\mathrm{H}_{2} \mathrm{O}_{2}, \mathrm{Na}_{2} \mathrm{MoO}_{4} \cdot 2 \mathrm{H}_{2} \mathrm{O}$ ethylene glycol $\mathrm{MeOH}$ $\mathrm{CH}_{3} \mathrm{CN}$ excess decomposition

ester exchanged ester exchanged no reaction

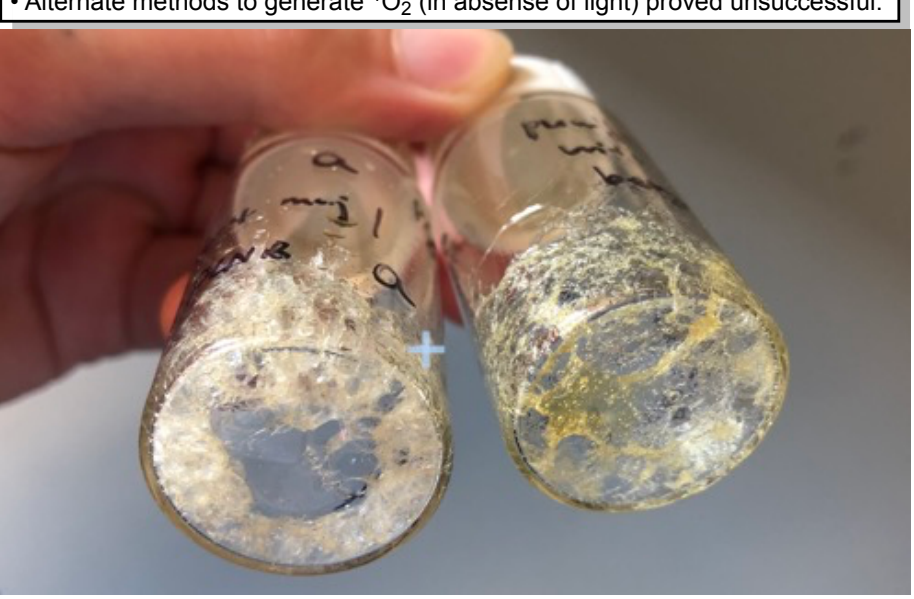

${ }^{*}$ Chiral auxiliary approach abandoned in favor of chiral $P(V)$ resolution approach following this observation (see 3rd-generation route)
*Absence of optical rotation data and configuration of natural isolate further supported this strategic reassessment

Scheme S4. Details regarding erosion of ee in oxidative rearrangement 


\section{Previous Strategies Towards Fully-functionalized Cyclopentane}

1) Epoxide opening strategy

First-generation strategy

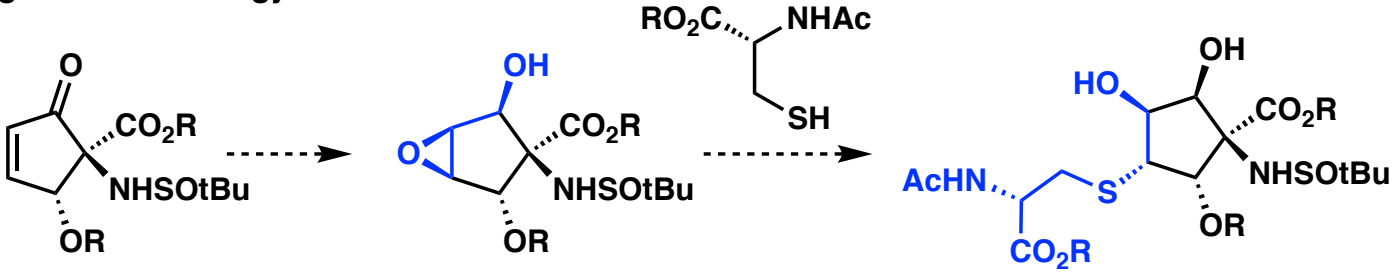

\section{Enone epoxidation}

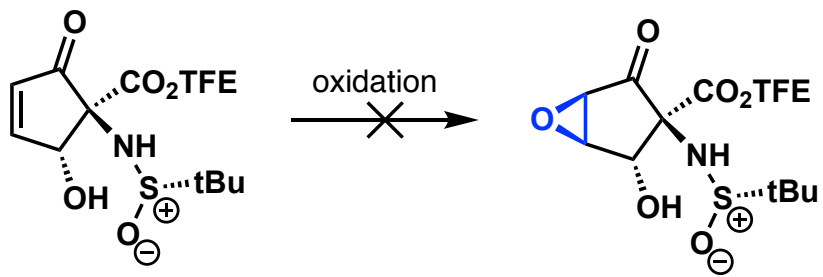

\begin{tabular}{ll}
\hline \multicolumn{1}{c}{ Conditions } & \multicolumn{1}{c}{ Results } \\
\hline $\mathrm{H}_{2} \mathrm{O}_{2}, \mathrm{NaOH}$ & complex mixture, ester hydrolysis \\
$\mathrm{mCPBA}, \mathrm{CH}_{2} \mathrm{Cl}_{2},-10^{\circ} \mathrm{C}$ & no reaction \\
$\mathrm{mCPBA}, \mathrm{CH}_{2} \mathrm{Cl}_{2}, \mathrm{rt}$ & {$[\mathrm{O}$ to sulfonamide only } \\
$\mathrm{mCPBA}, \mathrm{CH}_{2} \mathrm{Cl}_{2}, 40^{\circ} \mathrm{C}$ & {$[\mathrm{O}]$ to sulfonamide only } \\
$\mathrm{VO}(\mathrm{acac})_{2}, \mathrm{TBHP}^{\mathrm{PhH}} / \mathrm{CH}_{2} \mathrm{Cl}_{2},-10^{\circ} \mathrm{C}$ & {$[\mathrm{O}]$ to sulfonamide only (slow) } \\
$\mathrm{VO}(\mathrm{acac})_{2}, \mathrm{TBHP}, \mathrm{PhH}, 0^{\circ} \mathrm{C}$ & {$[\mathrm{O}$ to sulfonamide only } \\
$\mathrm{VO}(\mathrm{acac})_{2}, \mathrm{TBHP}, \mathrm{PhH}, \mathrm{rt}$ & {$[\mathrm{O}$ ] to sulfonamide only } \\
$\mathrm{Ti}(\mathrm{OiPr})_{4}, \mathrm{TBHP}, \mathrm{DET},-10^{\circ} \mathrm{C}$ & no reaction \\
$\mathrm{Ti}(\mathrm{OiPr})_{4}, \mathrm{TBHP}, \mathrm{DET}, \mathrm{rt}$ & {$[\mathrm{T}$ to sulfonamide only } \\
$\mathrm{DMDO}$, acetone & {$[\mathrm{O}$ to sulfonamide only } \\
$\mathrm{OsO}_{4}, \mathrm{tBuOH} / \mathrm{H}_{2} \mathrm{O}$ & {$[\mathrm{O}]$ to sulfonamide only } \\
\hline
\end{tabular}

\section{Epoxidation of allylic alcohol}

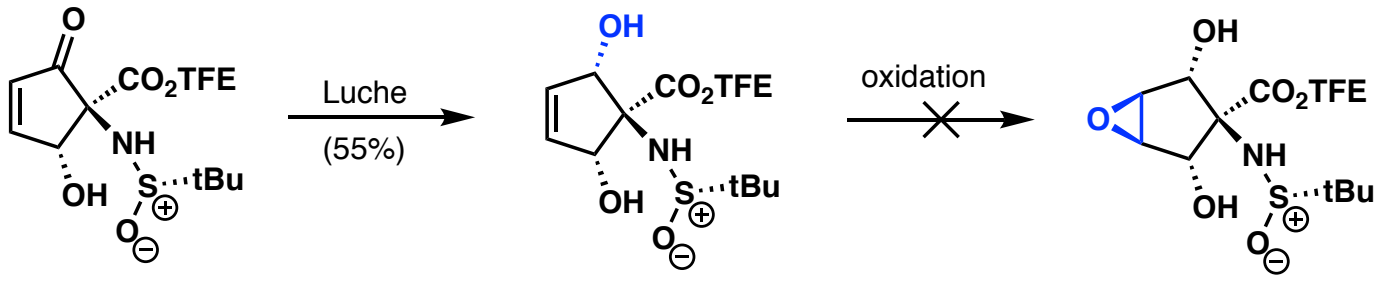

\begin{tabular}{|c|c|}
\hline Conditions & Results \\
\hline $\begin{array}{l}\mathrm{H}_{2} \mathrm{O}_{2}, \mathrm{NaOH} \\
\text { mCPBA, } \mathrm{CH}_{2} \mathrm{Cl}_{2},-10^{\circ} \mathrm{C} \\
\text { mCPBA, } \mathrm{CH}_{2} \mathrm{Cl}_{2}, \mathrm{rt} \\
\text { mCPBA, } \mathrm{CH}_{2} \mathrm{Cl}_{2}, 40^{\circ} \mathrm{C} \\
\text { VO(acac })_{2}, \mathrm{TBHP}^{\mathrm{P}} \mathrm{PhH} / \mathrm{CH}_{2} \mathrm{Cl}_{2},-10^{\circ} \mathrm{C} \\
\mathrm{VO}(\mathrm{acac})_{2}, \mathrm{TBHP}, \mathrm{PhH}, 0^{\circ} \mathrm{C} \\
\mathrm{VO}(\mathrm{acac})_{2}, \mathrm{TBHP}, \mathrm{PhH}, \mathrm{rt} \\
\mathrm{Ti}(\mathrm{OiPr})_{4}, \mathrm{TBHP}, \mathrm{DET},-10^{\circ} \mathrm{C} \\
\mathrm{Ti}(\mathrm{OiPr})_{4}, \mathrm{TBHP}, \mathrm{DET}, \mathrm{rt} \\
\text { DMDO, acetone } \\
\mathrm{OsO}_{4}, \mathrm{tBuOH} / \mathrm{H}_{2} \mathrm{O}\end{array}$ & $\begin{array}{l}\text { complex mixture, ester hydrolysis } \\
\text { no reaction } \\
\text { [O] to sulfonamide only } \\
\text { [O] to sulfonamide only } \\
\text { [O] to sulfonamide only (slow) } \\
\text { [O] to sulfonamide only } \\
\text { [O] to sulfonamide; some [O] to enone } \\
\text { no reaction } \\
\text { [O] to sulfonamide only } \\
{[\mathrm{O}] \text { to sulfonamide only }} \\
\text { [O] to sulfonamide only }\end{array}$ \\
\hline
\end{tabular}

Scheme S5. Epoxide-opening strategy towards cyclopentane functionalization 
2) Double displacement strategy Second-generation strategy

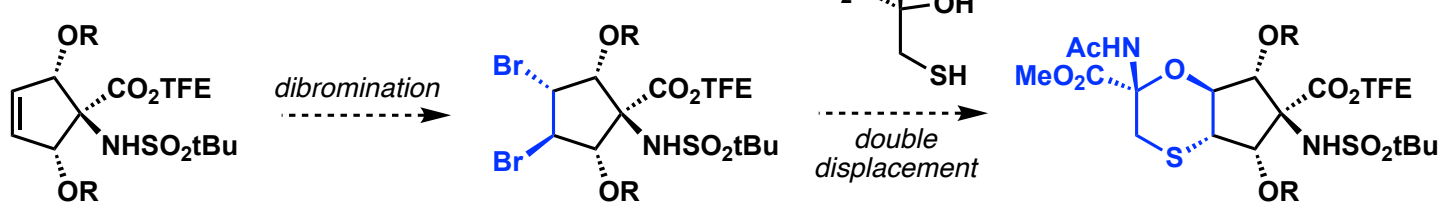

Dibromination
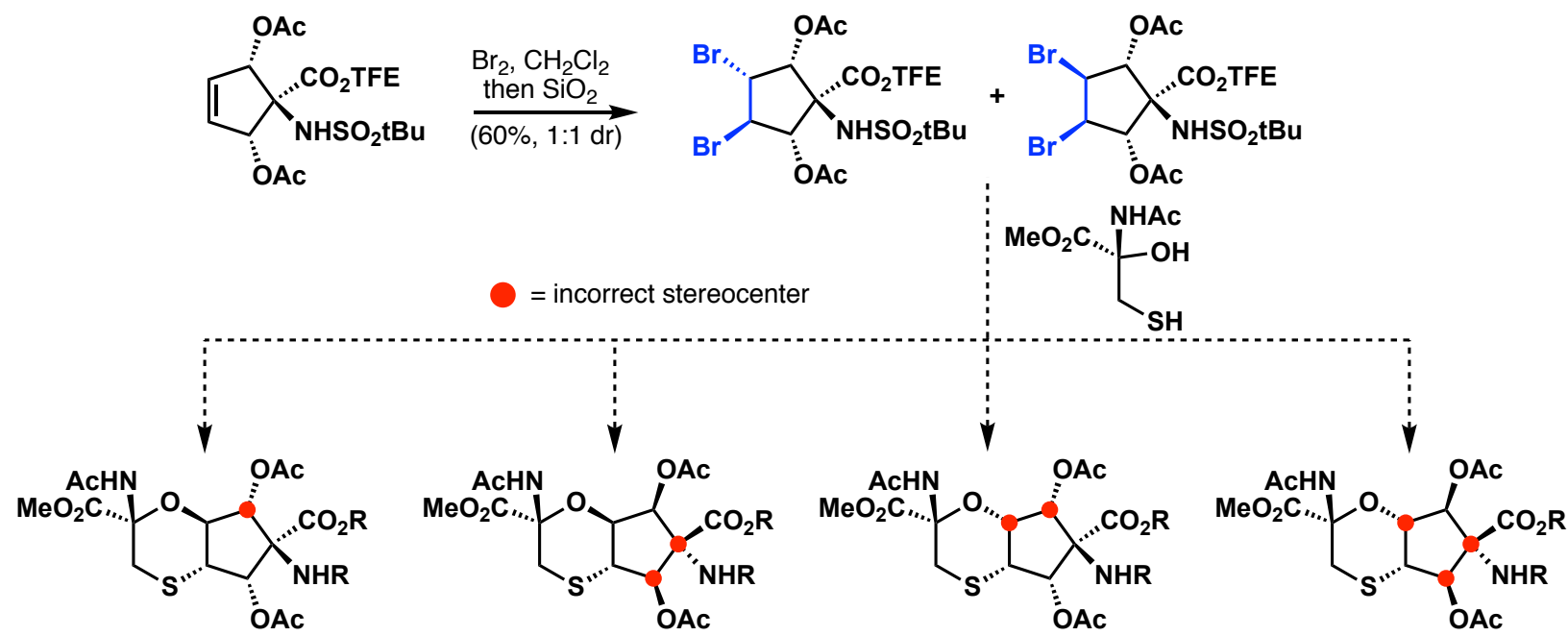

Double displacement would result in a mixture of diastereomers (for example as above) none with all correct stereochemistry. Thus, this approach was quickly abandoned.

Scheme S6. Double displacement strategy towards cyclopentane functionalization

3) Hetero-Michael addition strategy

Third-generation strategy

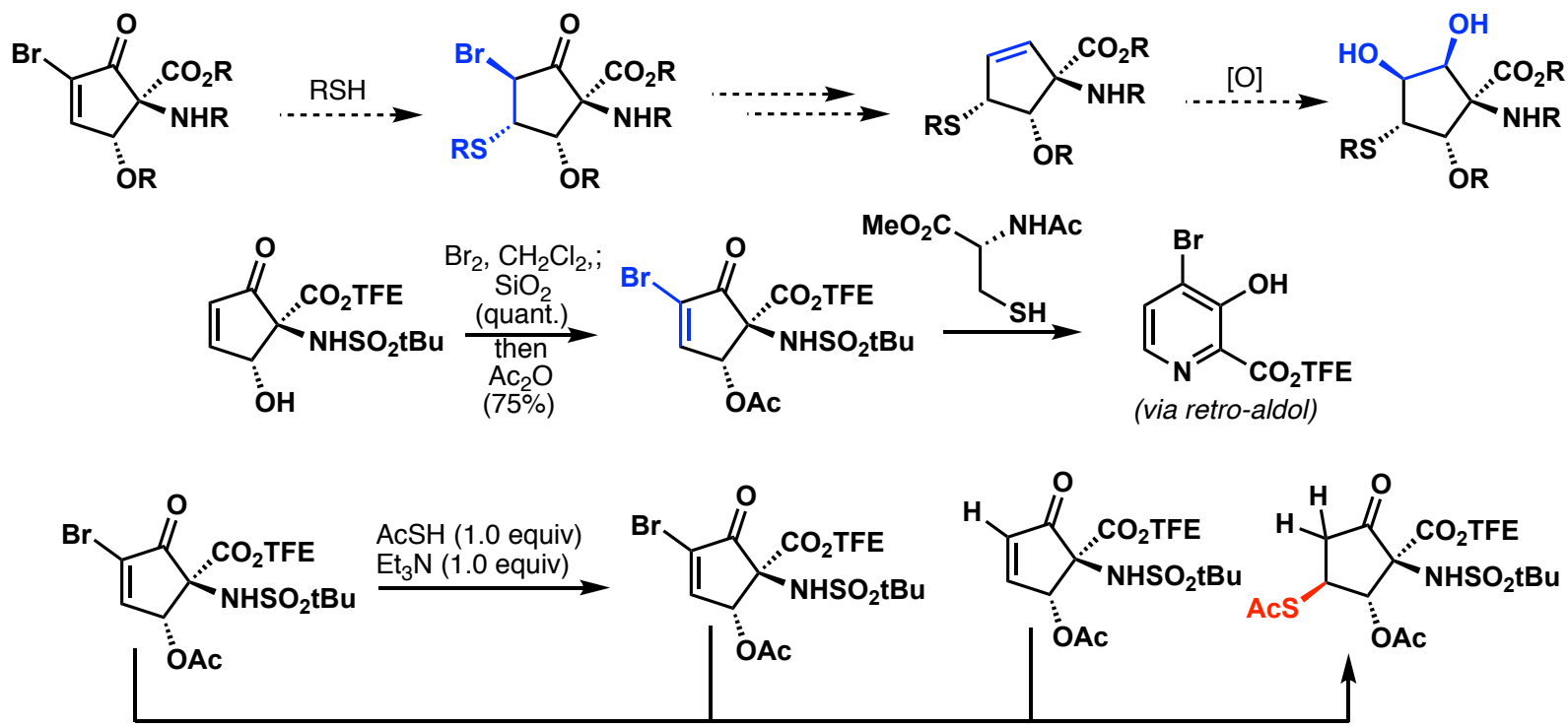

$\mathrm{AcSH}$ (10 equiv.), $\mathrm{Et}_{3} \mathrm{~N}$ (2.0 equiv.) (quant.)

NOE experiments confirmed incorrect sulfur stereochemistry, thus rendering this route untenable

Scheme S7. Hetero-Michael addition strategy towards cyclopentane functionalization 


\section{Debromination and Other Efforts}

a) Direct debromination

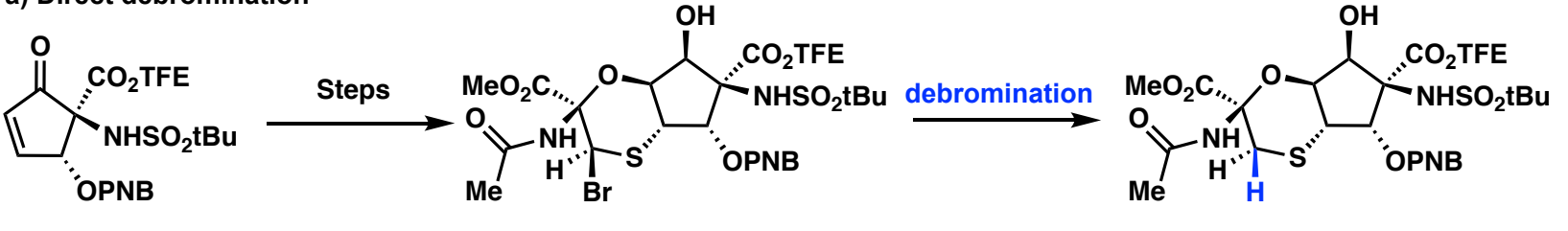

\begin{tabular}{|c|c|}
\hline Debromination Conditions & Results \\
\hline $\begin{array}{l}\text { - } \mathrm{Bu}_{3} \mathrm{SnH}, \mathrm{AIBN}, 110^{\circ} \mathrm{C}, 5 \mathrm{~h} \\
\text { - } \mathrm{Bu}_{3} \mathrm{SnH}, \mathrm{Et}_{3} \mathrm{~B} / \mathrm{O}_{2},-78^{\circ} \mathrm{C}, 2 \mathrm{~h} \\
\text { - } \mathrm{AIBN}, \mathrm{PhSiH}_{3}\left(\mathrm{as} \text { solvent), } 110^{\circ} \mathrm{C}, 1 \mathrm{~h}\right. \\
\text { - } \mathrm{AIBN},(\mathrm{TMS})_{3} \mathrm{SiH}, 80^{\circ} \mathrm{C}, 10 \mathrm{~min} \\
\text { - } \mathrm{Bu} \mathrm{SnH}_{3} \mathrm{SnH} \text { toluene, } 110^{\circ} \mathrm{C}, 1 \mathrm{~h} \\
\text { - toluene, } 110^{\circ} \mathrm{C} \text { (control experiment), } 3 \mathrm{~h} \\
\text { - } \mathrm{Bu}_{3} \mathrm{SnH} \& \mathrm{AIBN} \text { (slow addition over } 1.5 \mathrm{~h} \text { ), } \\
\text { benzene, } 85^{\circ} \mathrm{C}\end{array}$ & $\begin{array}{l}\text { very messy } \\
\text { decomposition } \\
\text { messy } \\
\text { decomposition } \\
\text { messy } \\
\text { no reaction (SM is heat stable) } \\
\text { desired product detected by HNMR (very low yield), } \\
\text { no SM remained }\end{array}$ \\
\hline 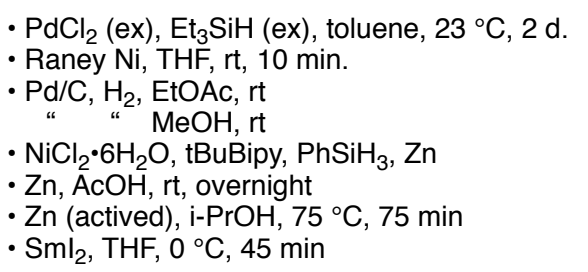 & $\begin{array}{l}\mathrm{Cl} \text { replacement } \\
\text { messy } \\
\text { no reaction } \\
\text { no reaction in } 30 \mathrm{~min} \text {; decomposed overnight } \\
\text { no reaction } \\
\text { messy } \\
\text { no reaction, became messy after longer heating } \\
\text { decomposition }\end{array}$ \\
\hline 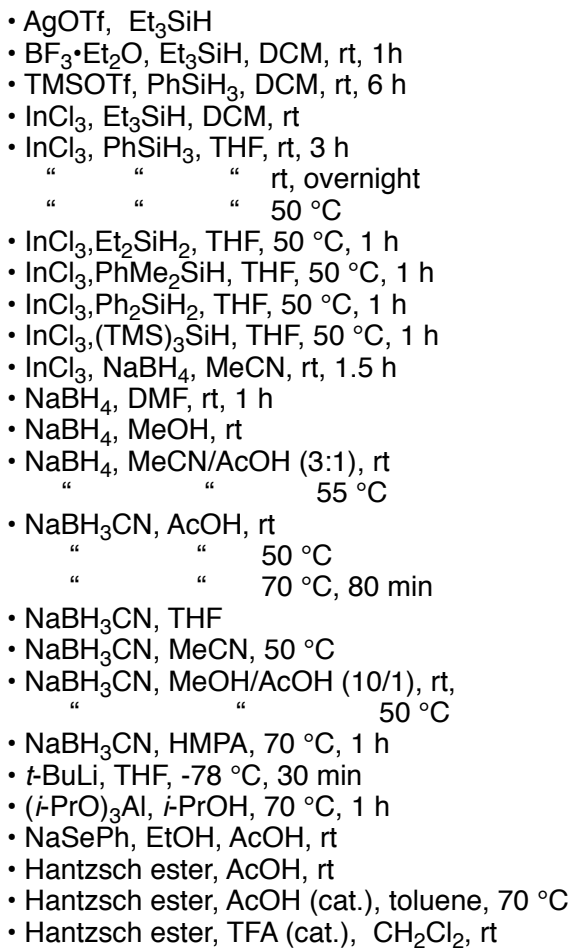 & $\begin{array}{l}\text { fast reaction, decomposition } \\
\text { almost only SM, trace of desired MS; became messy overnight } \\
\text { unknown product, } 50 \% \text { convertion } \\
\text { no reaction } \\
\text { SM as major, small amount of desired MS } \\
\text { messy } \\
\text { messy } \\
\text { messy } \\
\text { messy } \\
\text { messy } \\
\text { messy } \\
\text { messy } \\
\text { messy } \\
\text { decomposition } \\
\text { almost no reaction, trace of desired MS in LCMS } \\
\text { decomposition } \\
\text { no reaction } \\
\text { trace of desired MS in LCMS } \\
\text { trace of SM, more desired MS, but mostly decomposition } \\
\text { no reaction at rt, became messy after heating at } 50^{\circ} \mathrm{C} \\
\text { unknown product } \\
\text { almost no reaction } \\
\text { unknown product } \\
\text { decomposition } \\
\text { decomposition } \\
\text { SM + unknown product } \\
\text { decomposition } \\
\text { no reaction } \\
\text { no reaction } \\
\text { no reaction }\end{array}$ \\
\hline
\end{tabular}

Table S1. Conditions for direct debromination 
b) Protection of the secondary alcohol imparted stability

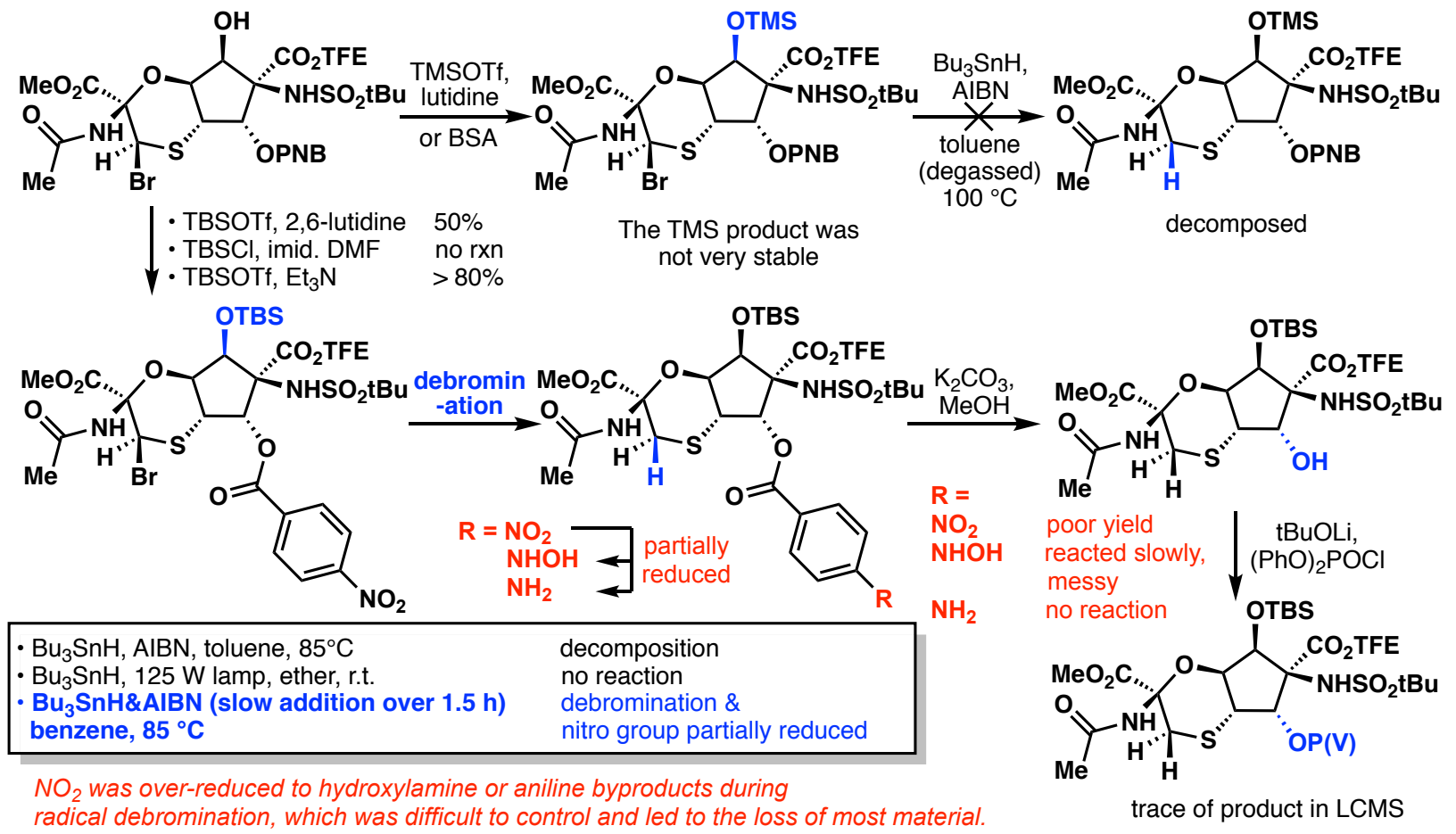

c) Other efforts
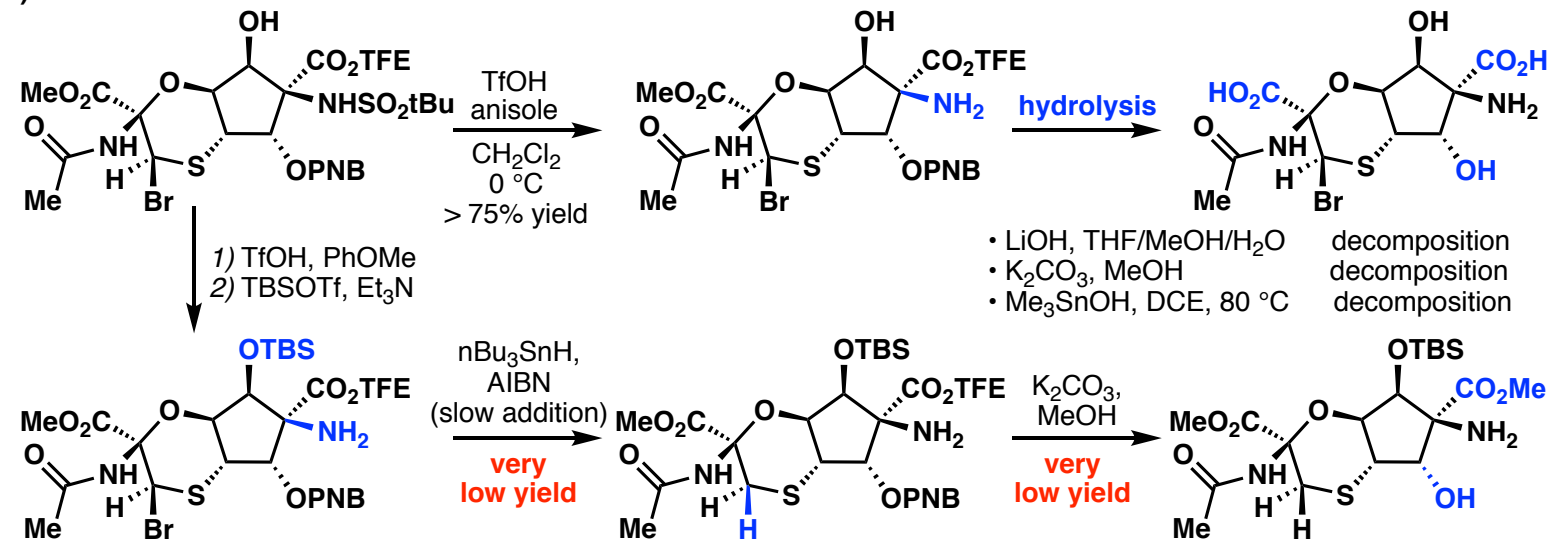

Low efficiency and difficulties in debromination and PNB deprotection necessitated changing the alcohol protecting group.

Scheme S8. Debromination and other efforts 


\section{III) Summary of Second-Generation Synthesis Optimization}

Second-generation route:

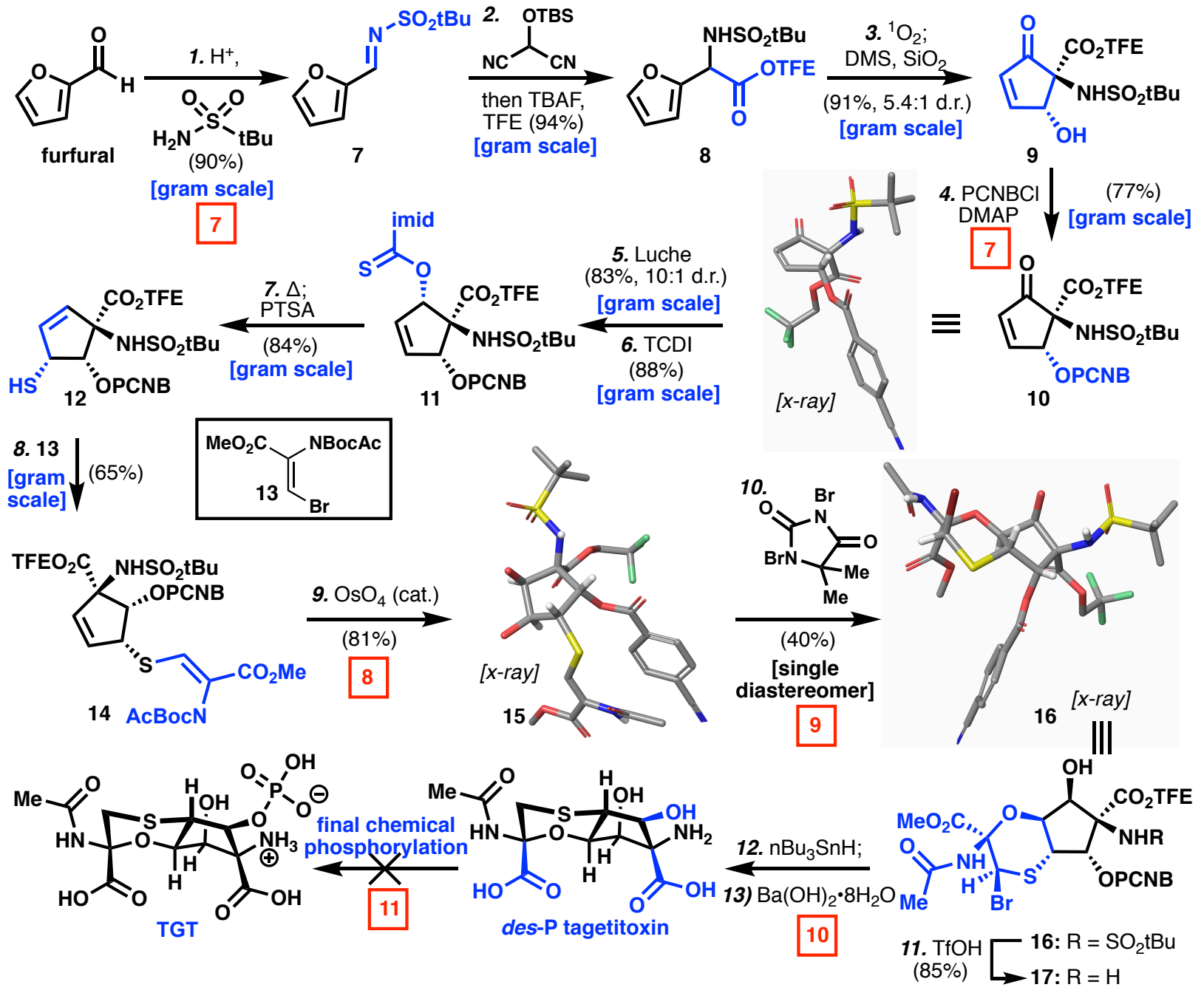

Reaction Optimization:

7 - Advantages of using sulfonamide and $p$-CN benzoyl (PCNB) protecting group (p. SI-12)

8 - Dihydroxylation (p. SI-13)

9 - Construction of trans-[6,5]-1,4-oxathiane heterocycle (p. SI-16)

10 - Global hydrolysis (p. SI-21)

11 - Unsuccessful selective phosphorylation (p. SI-22)

Key Challenges:

11 - The solubility problem of des-P tagetitoxin.

Scheme S9. Second-generation route to des-P tagetitoxin 
7 - Advantages of using sulfonamide and $p$-CN benzoyl (PCNB) protecting group

Advantages of using sulfonamide:
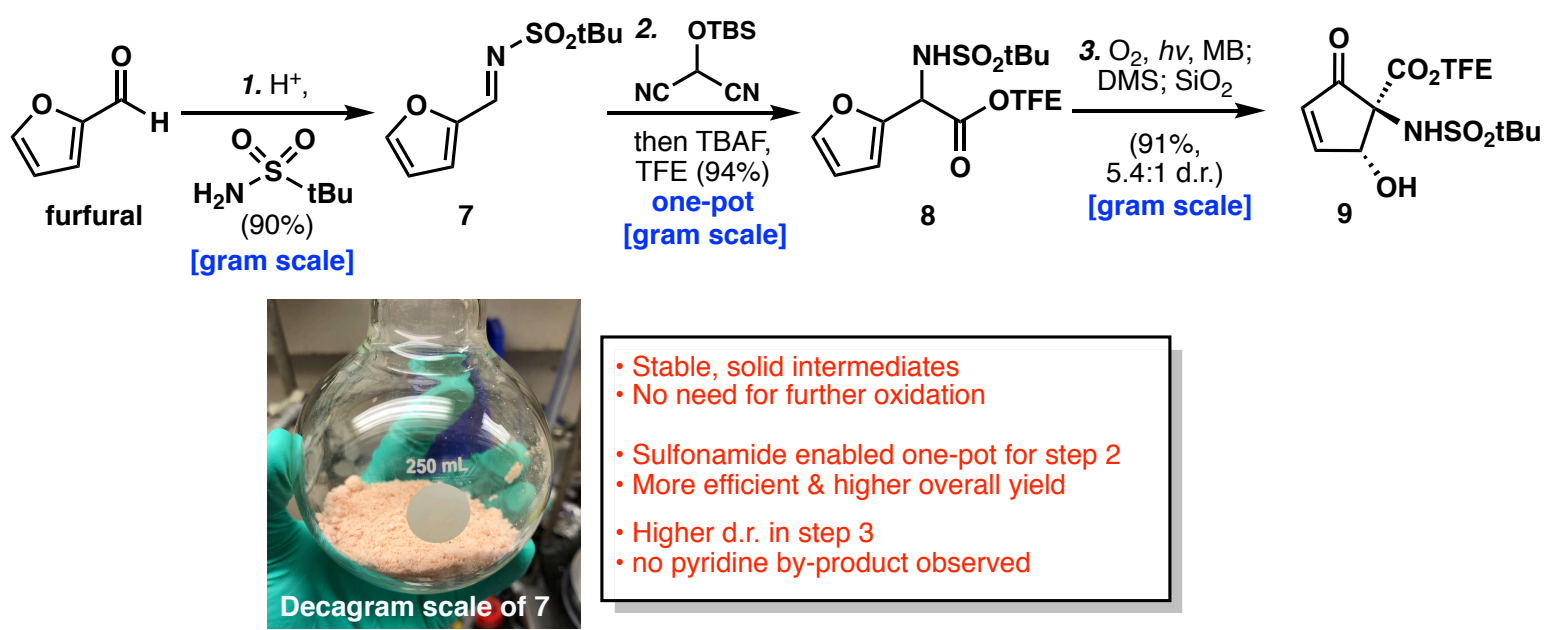

- Stable, solid intermediates

- No need for further oxidation

- Sulfonamide enabled one-pot for step 2

- More efficient \& higher overall yield

- Higher d.r. in step 3

- no pyridine by-product observed

Advantages of using $p-C N$ benzoyl (PCNB) protecting group:

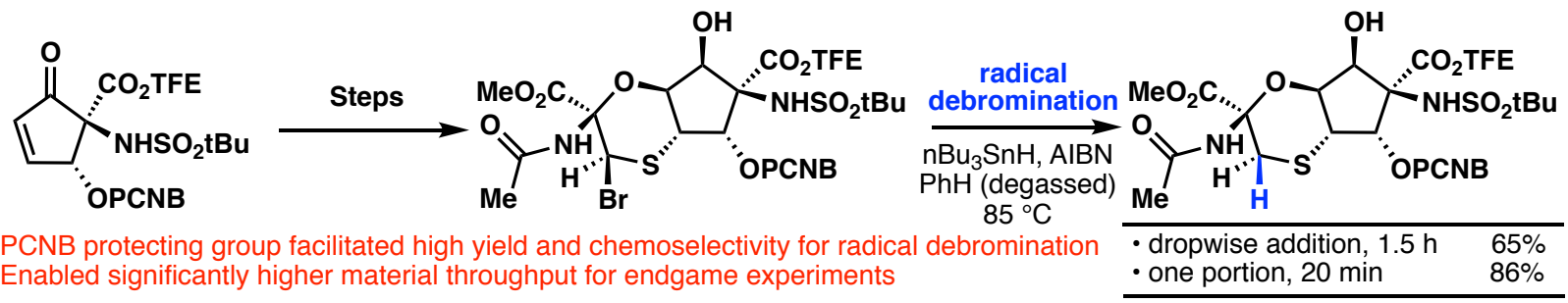

Scheme S10. Advantages of using sulfonamide and PCNB protecting group 
8 - Dihydroxylation

a) Dihydroxylation after thio-[3,3]-rearrangement

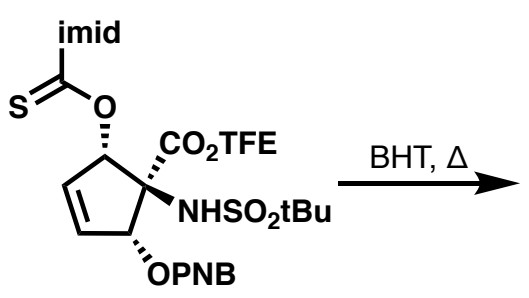

\section{Conditions}

- $\mathrm{OsO}_{4}$ (cat.)

- $\mathrm{OsO}_{4}$, pyridine, $\mathrm{CH}_{2} \mathrm{Cl}_{2}$, rt

- $\mathrm{OsO}_{4}$ (cat.), NMO, DMAP, tBuO acetone/tBuOH/ $/ \mathrm{H}_{2} \mathrm{O}, 45^{\circ} \mathrm{C}$<smiles>CCO[Nb][C@]1(C(=O)OCC)C=C[C@@H](SC(=O)CC)[C@@H]1O[Pb]</smiles>

Results

no reaction slowly decomposed decomposition

enone $\mathbf{A}$

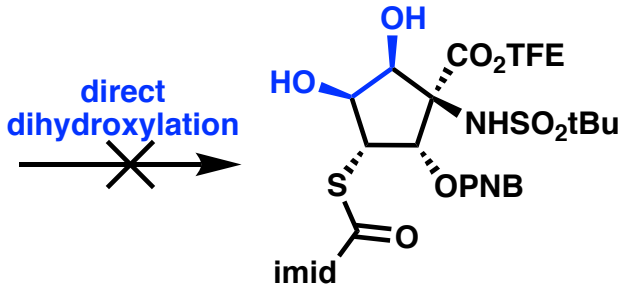

imid

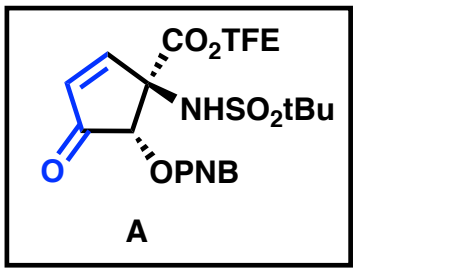

Stoichiometric osmylation; osmate cleavage optimization

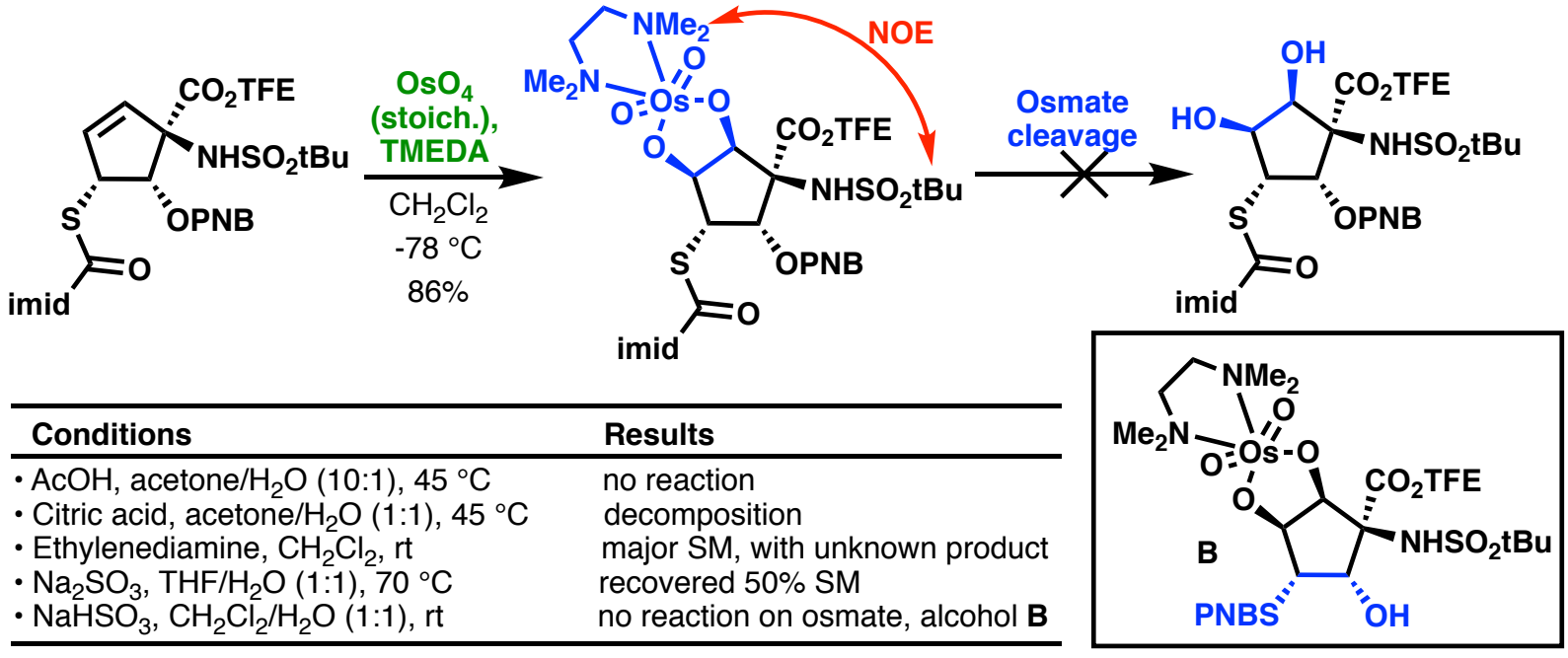

Scheme S11. Dihydroxylation efforts on product of $[3,3]$-sigmatropic rearrangement 
b) Dihydroxylation after sidechain installation

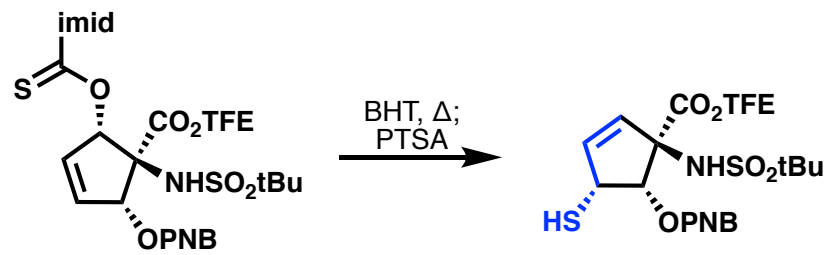

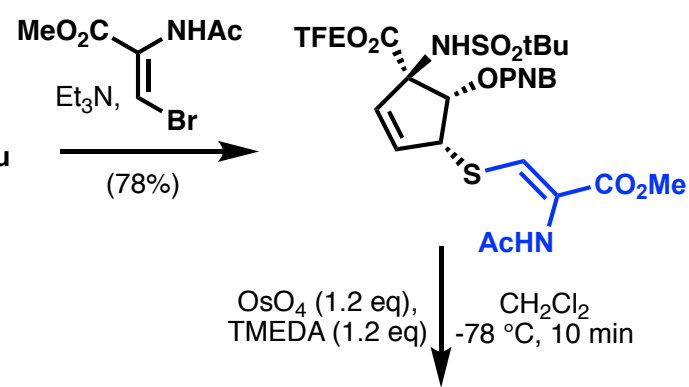

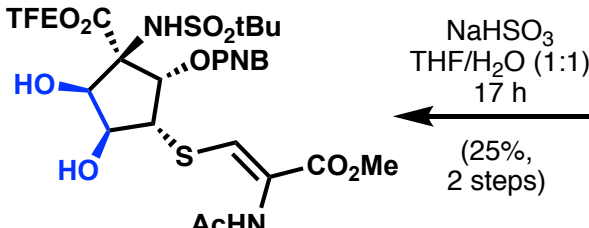

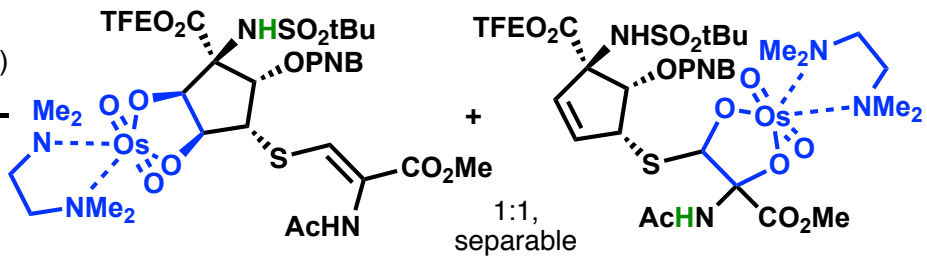

- No olefin selectivity

- Low yield

- Not scalable (50 mg max)

$\mathrm{N}$-Boc analog of amino acid sidechain engendered complete dihydroxylation chemoselectivity.

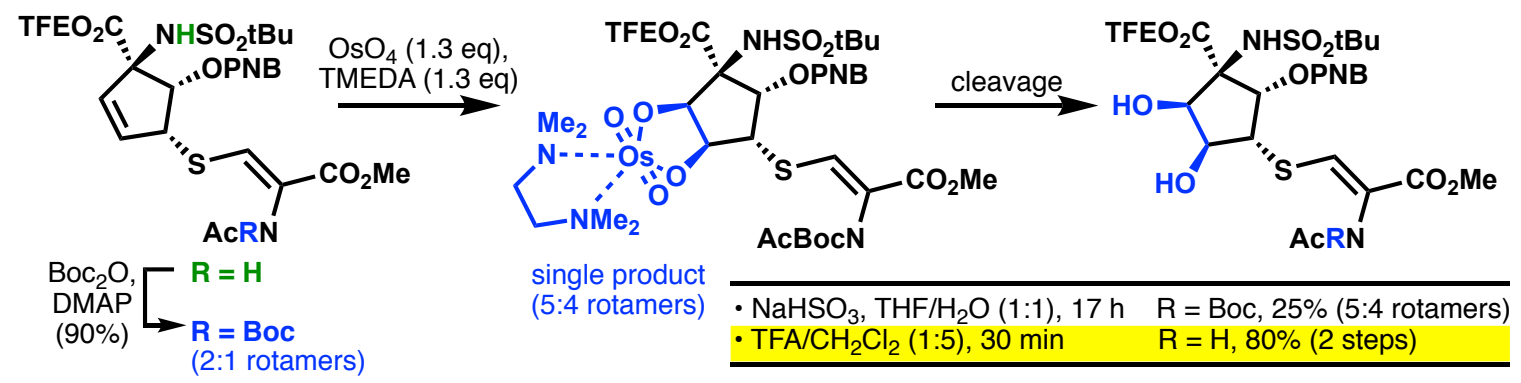

Directly obtained desired intermediate with Boc protected vinyl bromide:

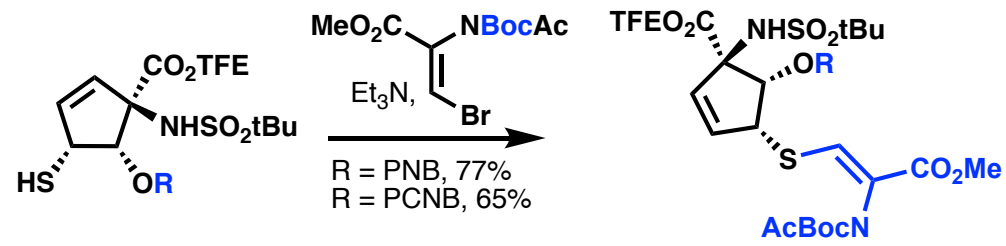

c) Catalytic dihydroxylation:

Attempts at using SM without Boc on sidechain
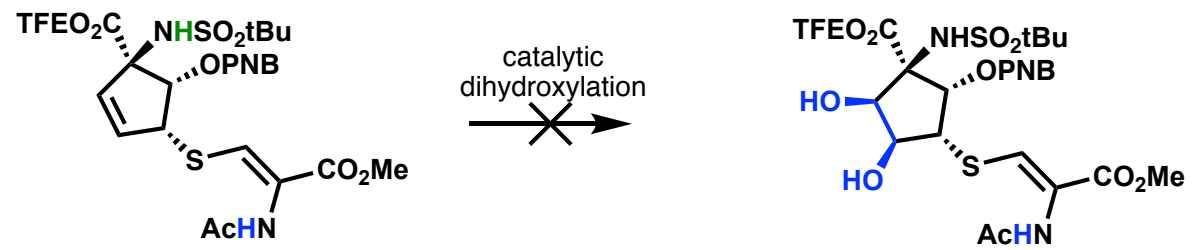

- $\mathrm{OsO}_{4}$ (0.1 equiv), $\mathrm{NMO}$, citric acid, tBuOH/acetone $/ \mathrm{H}_{2} \mathrm{O}(1: 1: 1), 35^{\circ} \mathrm{C}$

- $\mathrm{OsO}_{4}$ (0.1 equiv), $\mathrm{NMO}$, citric acid, tBuOH/acetone/ $\mathrm{H}_{2} \mathrm{O}(1: 1: 1)$, rt

very messy

very messy

Scheme S12. Dihydroxylation optimization details 
Attempts at using SM with Boc on sidechain
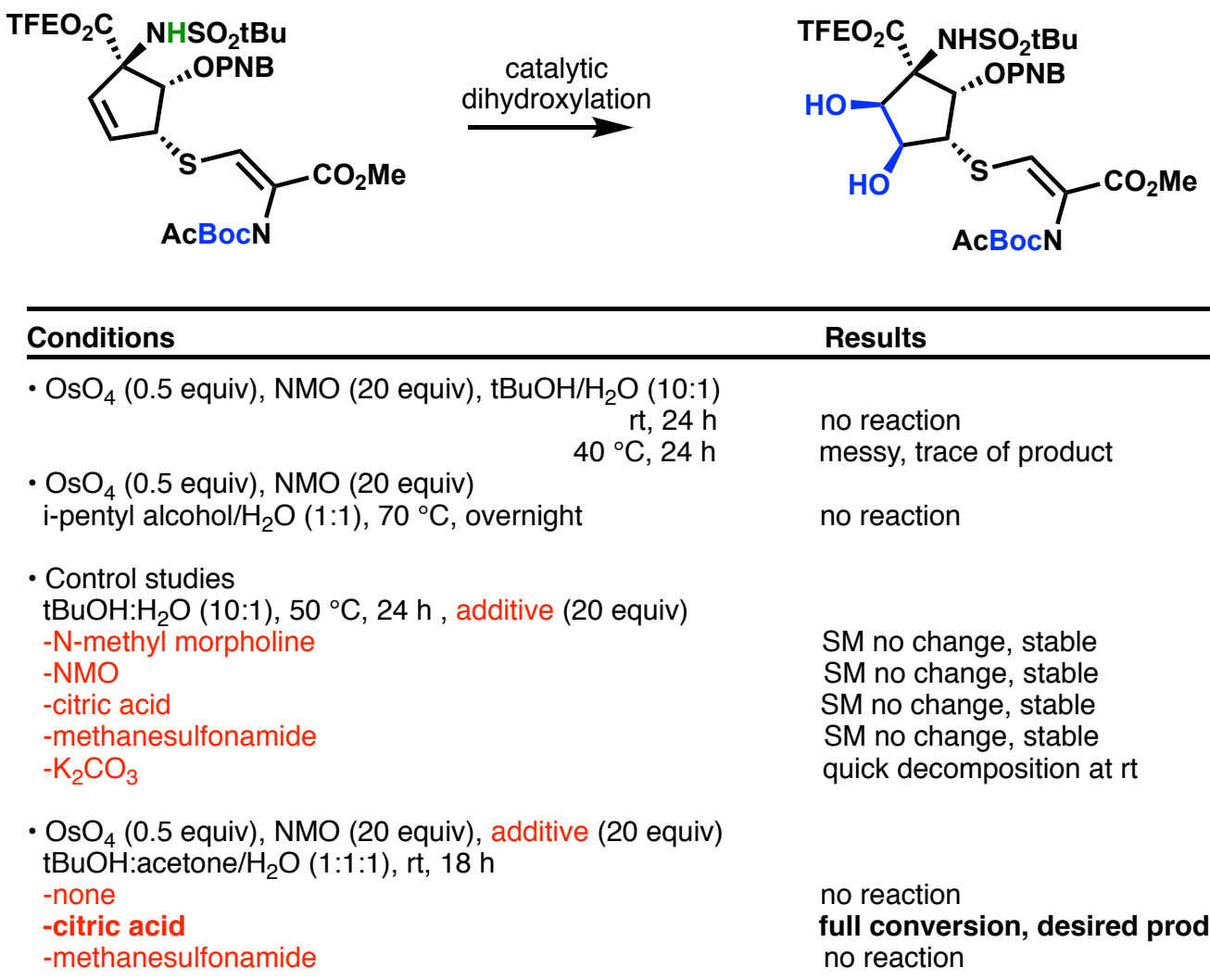

Results

no reaction

messy, trace of product

no reaction

SM no change, stable SM no change, stable SM no change, stable SM no change, stable quick decomposition at it

no reaction

full conversion, desired product in LCMS no reaction

- $\mathrm{OsO}_{4}$ (X equiv), NMO (20 equiv), citric acid (20 equiv) $\mathrm{tBuOH}$ :acetone/ $\mathrm{H}_{2} \mathrm{O}(1: 1: 1)$, rt

-0.4 equiv, $24 \mathrm{~h}$

$97 \%$ product (by LCMS)

-0.3 equiv, $24 \mathrm{~h}$

$40 \mathrm{~h}$

$85 \%$ product (by LCMS)

$97 \%$ product (by LCMS)

$85 \%$ product (by LCMS)

-0.2 equiv, $24 \mathrm{~h}$

$40 \mathrm{~h}$

-0.1 equiv, $24 \mathrm{~h}$

$97 \%$ product (by LCMS)

$61 \%$ product (by LCMS)

$40 \mathrm{~h}$

$81 \%$ product (by LCMS)

4 days

$\mathbf{9 7 \%}$ product (by LCMS)

- $\mathrm{OsO}_{4}$ (0.1 equiv), NMO (10 equiv), citric acid (10 equiv)

tBuOH:acetone/ $\mathrm{H}_{2} \mathrm{O}(1: 1: 1), 35^{\circ} \mathrm{C}, 14 \mathrm{~h}, 25 \mathrm{mg}$ scale

$80 \%$ isolated yield

Combined with acid work up
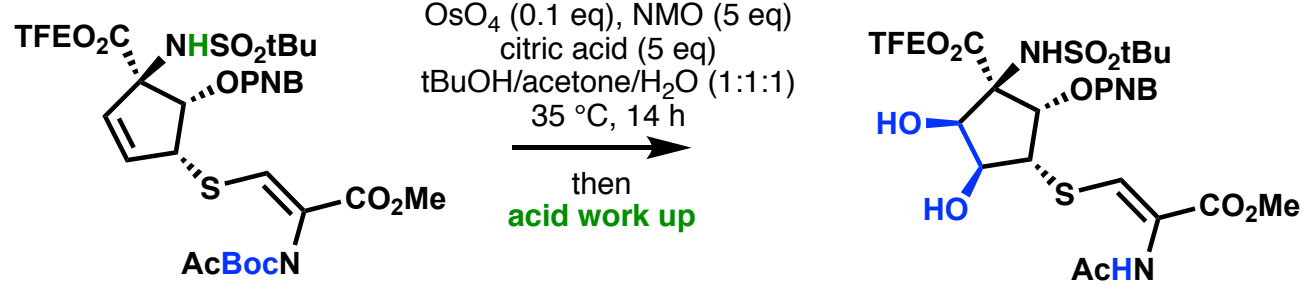

- $80 \% \mathrm{AcOH}, 48 \mathrm{~h}$

no reaction

-xs TFA, $24 \mathrm{~h}$

- Solvent qty. TFA, $24 \mathrm{~h}$

no reaction

- Solvent qty. conc. $\mathrm{HCl}, 30 \mathrm{~min}$

full conversion

$200 \mathrm{mg}$ scale, $81 \%$ yield

Scheme S13. Catalytic dihydroxylation details 


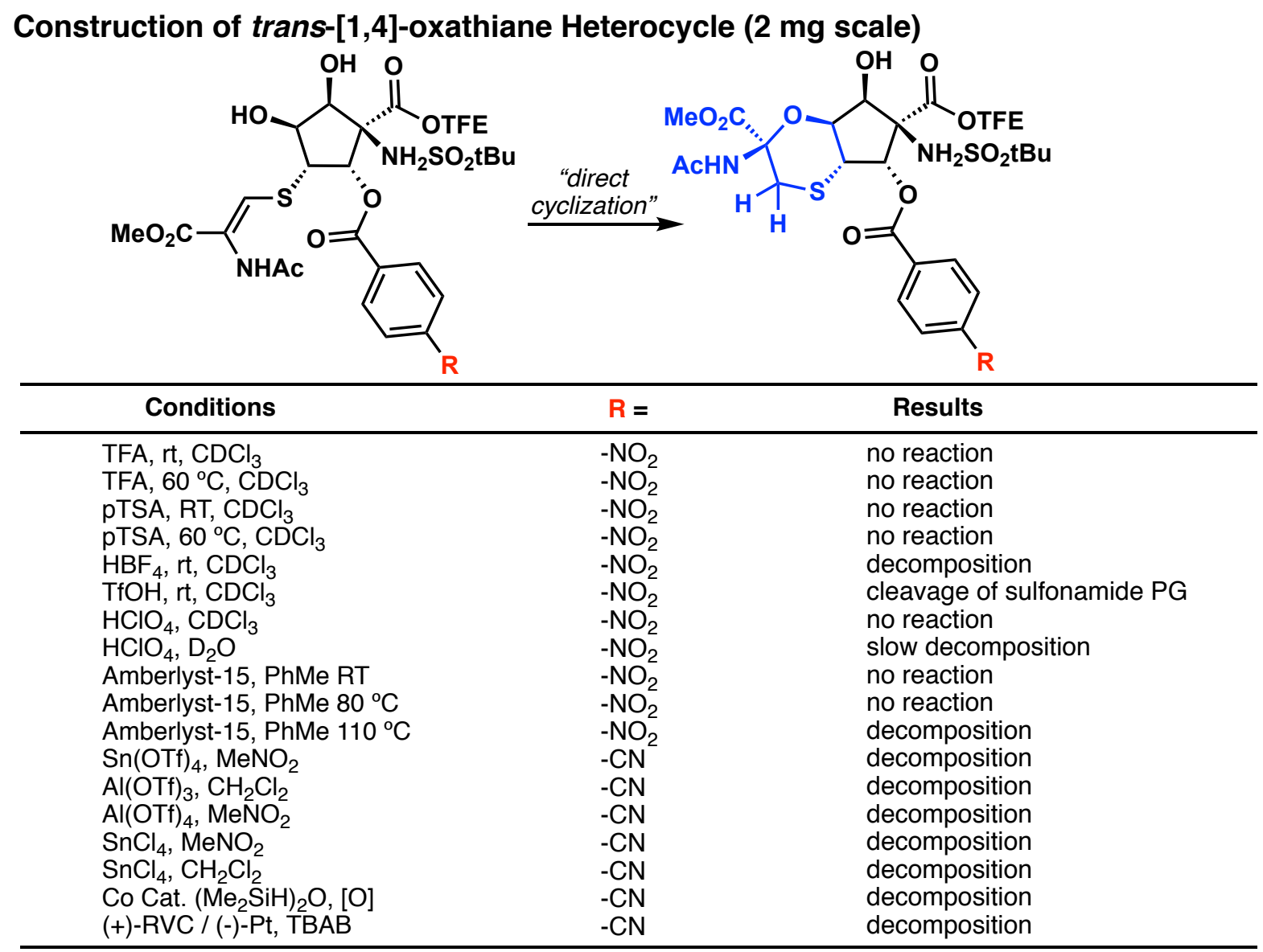

Table S2. Attempts at direct cyclization towards trans-[6,5]-1,4-oxathiane 


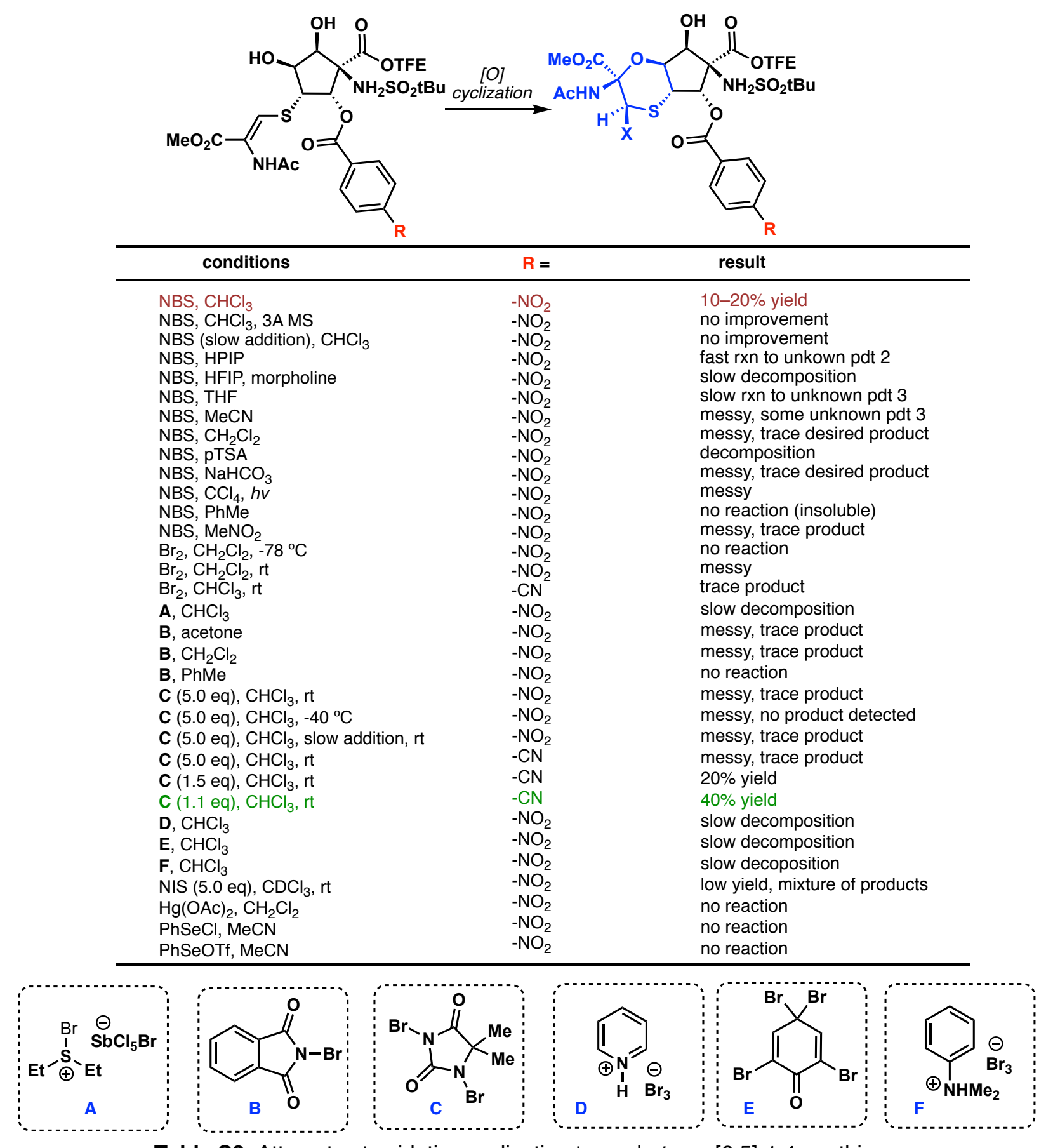

Table S3. Attempts at oxidative cyclization towards trans-[6,5]-1,4-oxathiane 


\section{Further optimization of bromoetherification}

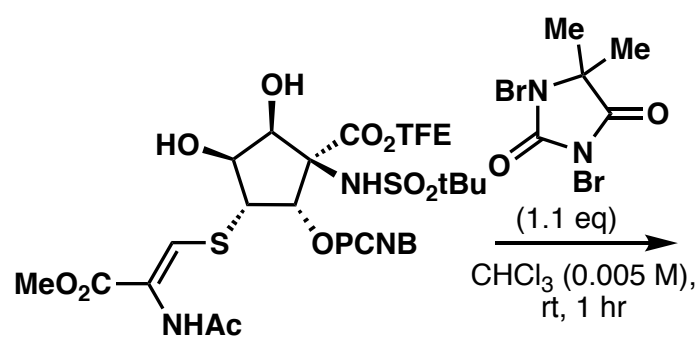

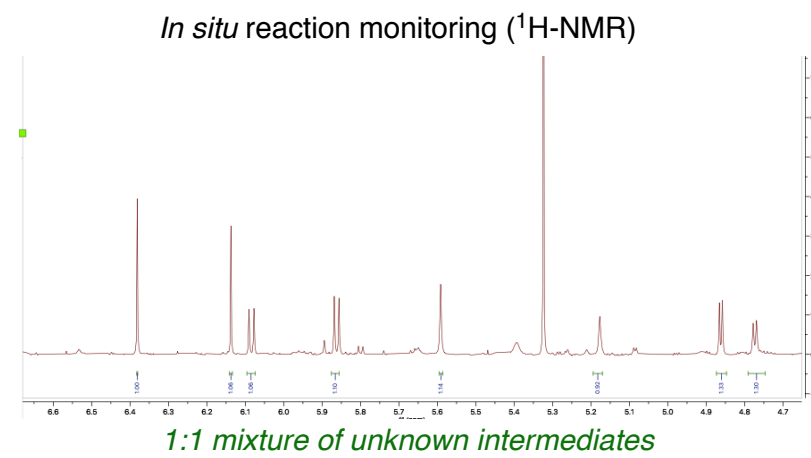

Initial hypotheses regarding identity of intermediates:

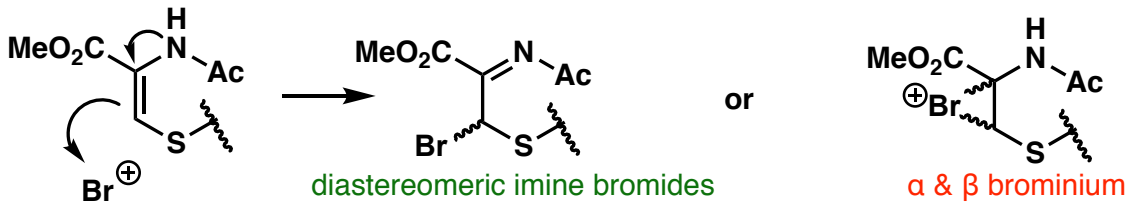

Evidence 1: Lack of reactivity when $\mathrm{N}$-substituents present suggests non-bonding nitrogen electrons play a role

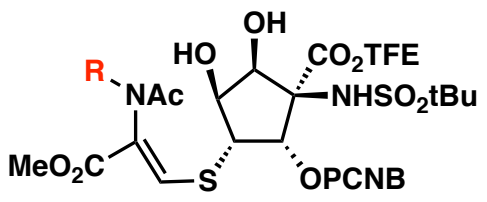

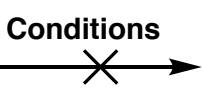

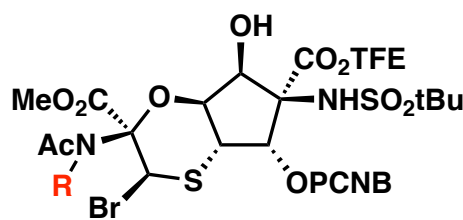

Table S5.

\begin{tabular}{|c|c|c|}
\hline Conditions & $\mathbf{R}=$ & Results \\
\hline 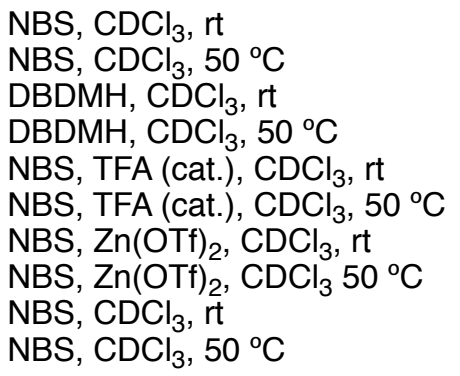 & $\begin{array}{l}-B o c \\
-B o c \\
-B o c \\
-B o c \\
-B o c \\
-B o c \\
-B o c \\
-B o c \\
-M e \\
-M e\end{array}$ & $\begin{array}{l}\text { no reaction } \\
\text { no reaction } \\
\text { no reaction } \\
\text { no reaction } \\
\text { no reaction } \\
\text { no reaction } \\
\text { no reaction } \\
\text { no reaction } \\
\text { no reaction } \\
\text { no reaction } \\
\text { no reaction }\end{array}$ \\
\hline
\end{tabular}

Nitrogen plays a crucial role in this transformation

Evidence 2: Regardless of exact mechanism, intermediate is chemically-similar to desired product
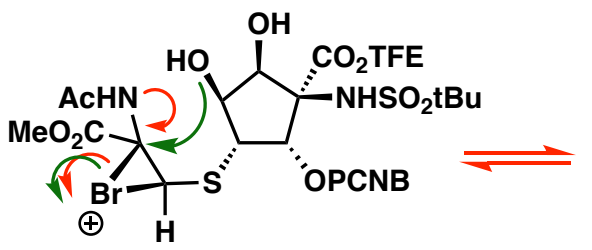

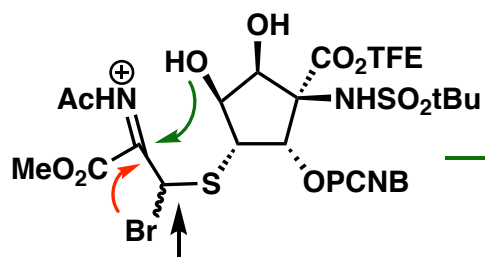

${ }^{13} \mathrm{C}$ ठ 50.84, $50.11 \mathrm{ppm}$

(from HC/HSQC study)

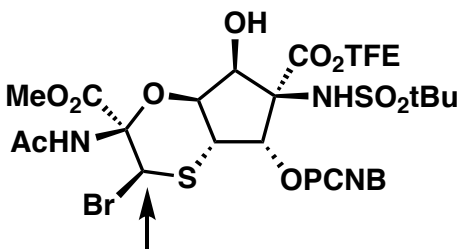

${ }^{13} \mathrm{C} \delta 56.47 \mathrm{ppm}$

Evidence 3: No reports of stable bromoniums adjacent to heteroatoms ${ }^{[2]}$

Conclusion: diastereomeric imine bromides likely to be the observed intermediates

Scheme S14. Experiments towards understanding reactive intermediates 
Attempted pre-installation of bromide, then simultaneous acetonide deprotection, protonation, ring closure

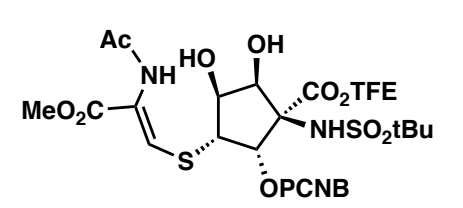

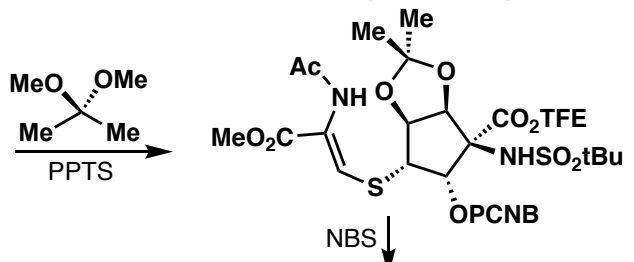

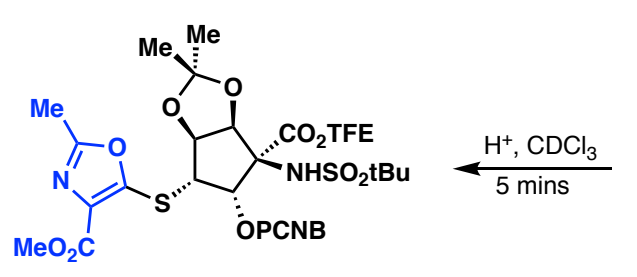

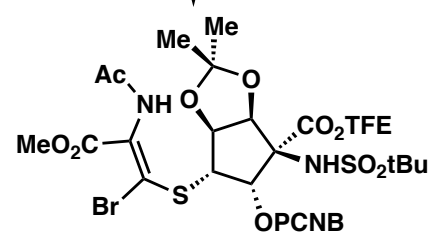

Conclusions:

Observed intermediate is highly reactive (as evidenced by rapid oxazole formation) Longer reaction times (a function of concentration and temperature) are deleterious to reaction efficiency

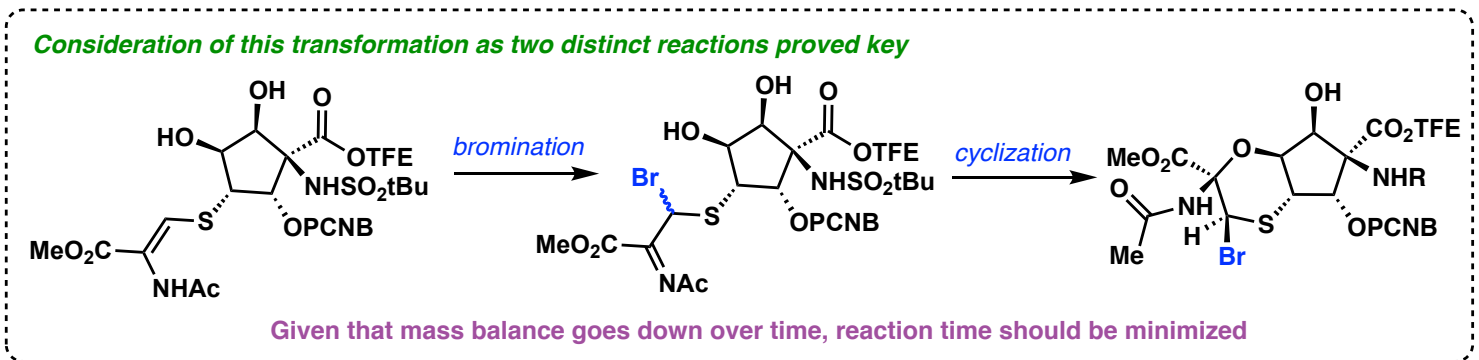

More variables for bromination...

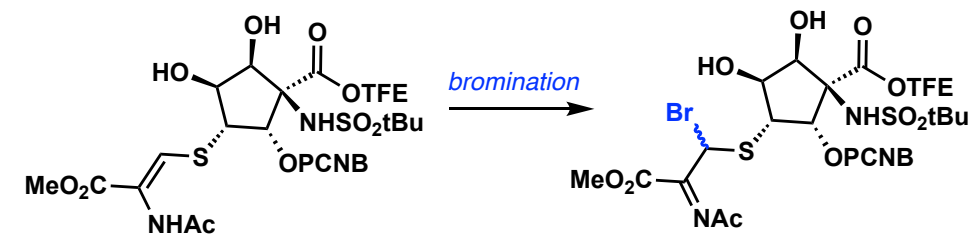

Concentration effects ( $6 \mathrm{mg}$ scale) on bromination \& subsequent stability / yield

$\begin{array}{lll}\text { Concentration } & \text { Reaction time } & \text { Product Yield (*see cyclization optimization) } \\ 0.0015 \mathrm{M} & 60 \text { mins } & 25-35 \% \\ 0.015 \mathrm{M} & 15 \mathrm{mins} & 30-35 \% \\ 0.5 \mathrm{M} & 3 \mathrm{mins} & 40-45 \%\end{array}$

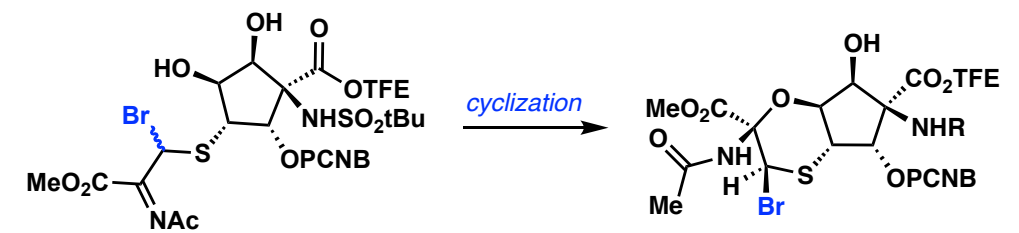

$$
\begin{array}{ll}
0.5 \mathrm{M}, 30 \mathrm{mins}, 50^{\circ} \mathrm{C} & 20-35 \% \text { (messy) } \\
0.5 \mathrm{M}, 1 \mathrm{~min}, 100^{\circ} \mathrm{C} & 20-35 \% \text { (messy) } \\
0.015 \mathrm{M}, 30 \mathrm{mins}, 50{ }^{\circ} \mathrm{C} & 45 \% \\
0.015 \mathrm{M}, 1 \mathrm{~min}, 100^{\circ} \mathrm{C} & 40 \% \\
0.0015 \mathrm{M}, 30 \mathrm{~min}, 50^{\circ} \mathrm{C} & \text { minimal product (messy) }
\end{array}
$$

Control of concentration / lifetime of unstable intermediate(s) rendered this reaction scalable

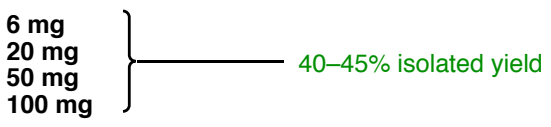

Scheme S15. Improving scalability / robustness of bromoetherification 
Working mechanistic hypothesis for stereochemical outcome:

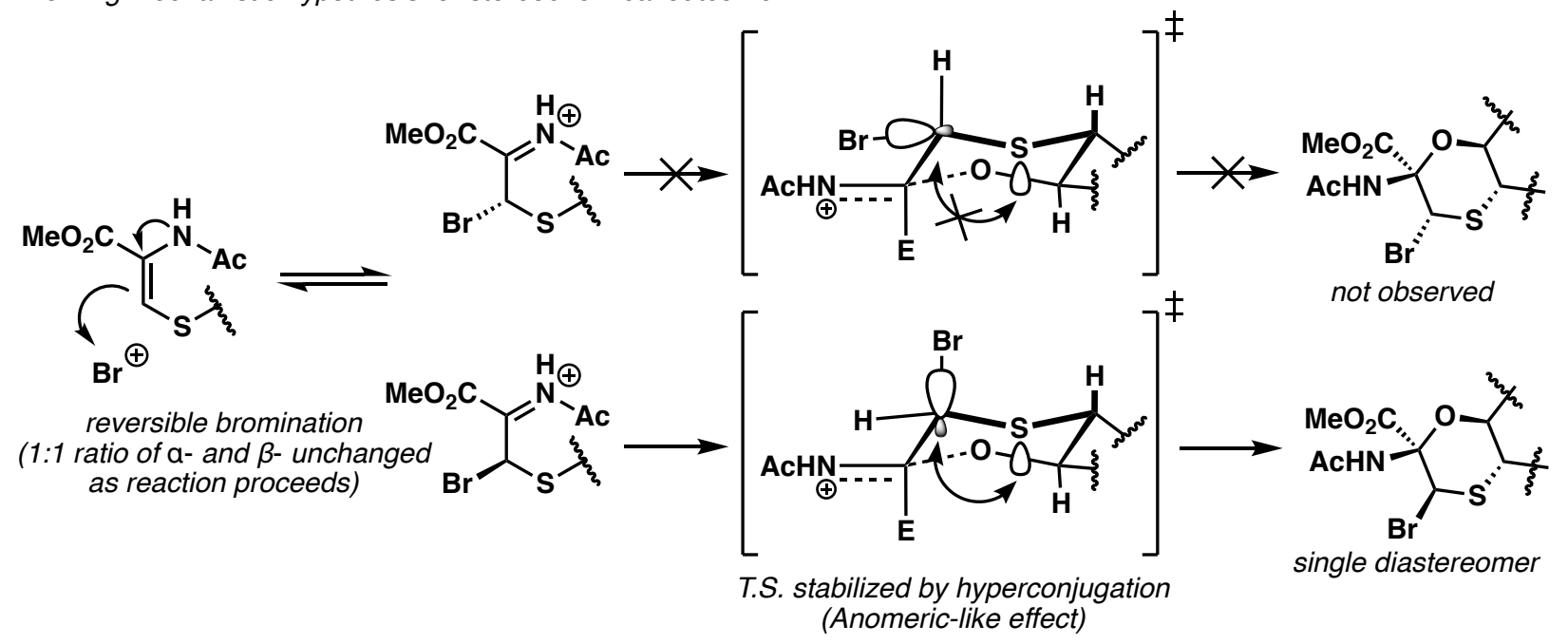

Scheme S16. Current mechanistic hypothesis / stereochemical model 
10 - Global hydrolysis

Synthesis of des-P tagetitoxin (selective phosphorylation as last step)
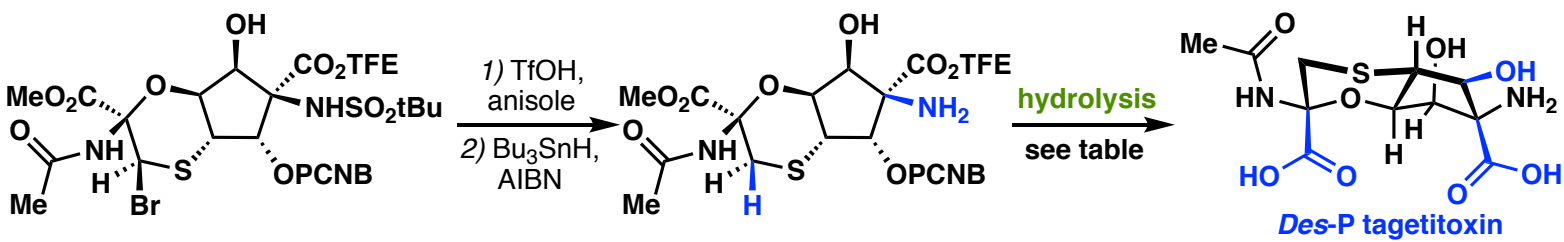

Des-P tagetitoxin

\begin{tabular}{|c|c|}
\hline Hydrolysis ( $0.2 \mathrm{mg}$ scale): & Results \\
\hline 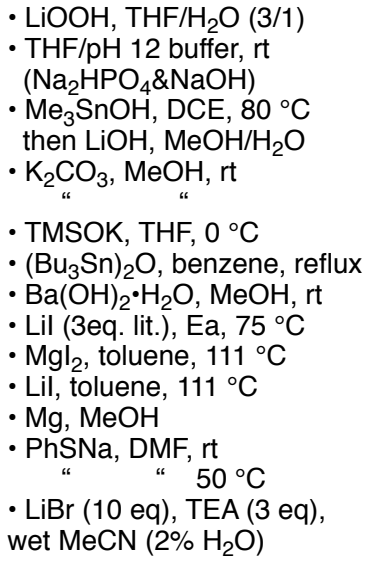 & $\begin{array}{l}\text { decomposition } \\
\text { decomposition } \\
\text { only TFE ester hydrolyzed } \\
\text { decomposition } \\
10 \text { min, TFE ester exchanged first, PCNB deprotected; } \\
25 \text { min, messy } \\
\text { decomposition } \\
\text { no reaction } \\
\text { decomposition } \\
\text { TFE and methyl esters hydrolyzed, low yield } \\
\text { decomposition } \\
\text { decomposition } \\
\text { decomposition } \\
\text { no reaction } \\
\text { no reaction } \\
\text { decomposition }\end{array}$ \\
\hline - $\mathrm{Ba}(\mathrm{OH})_{2} \cdot 8 \mathrm{H}_{2} \mathrm{O}, \mathrm{D}_{2} \mathrm{O}, \mathrm{rt}$ & $\begin{array}{l}\text { 1.5h, fully hydrolyzed, des-P tagetitoxin, } \\
\text { H-NMR shift and } J_{2-2} \text {, and } J_{6-7} \text { are same as NP in lit.!! }\end{array}$ \\
\hline
\end{tabular}

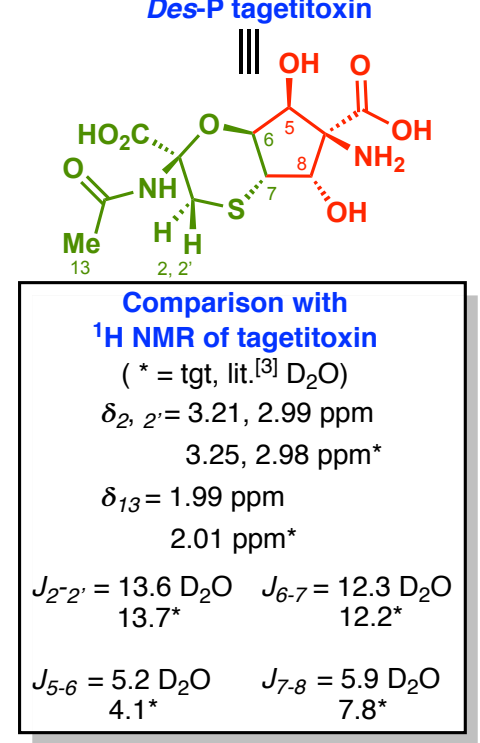

Easy work up procedure after fully hydrolyzed with $\mathrm{Ba}(\mathrm{OH})_{2}$ :

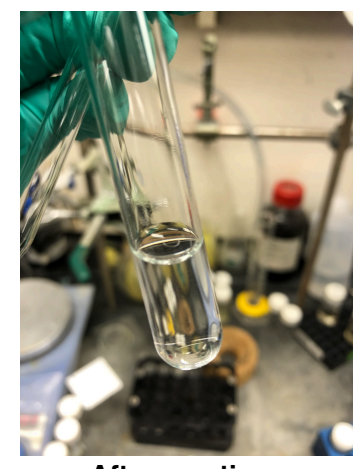

After reaction:

$\mathrm{Ba}(\mathrm{OH})_{2}\left(0.1 \mathrm{M}\right.$ in $\mathrm{D}_{2} \mathrm{O}, 10$ eq. $\mathrm{D}_{2} \mathrm{O}, \mathrm{rt}, 50 \mathrm{~min}$

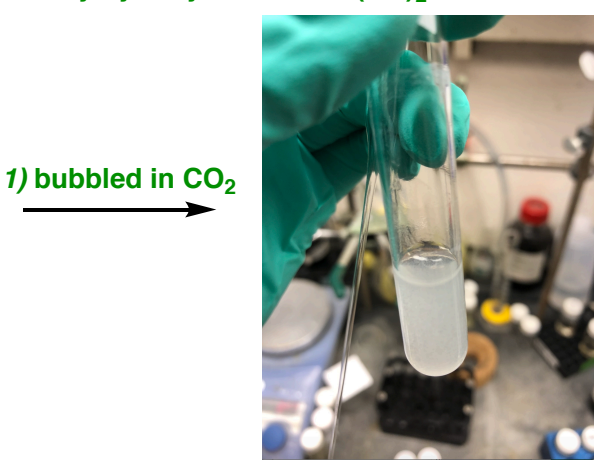

White $\mathrm{BaCO}_{3}$ precipitated out
2) filtered \& lyophilized

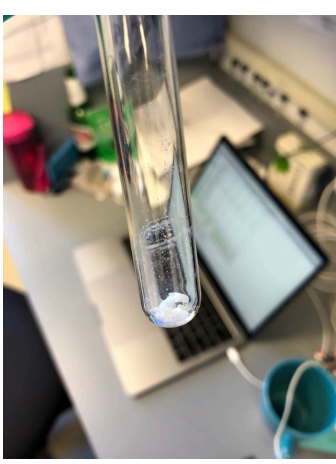

Des-P tagetitoxin white and clean powder

Scheme S17. Synthesis of des-P tagetitoxin 
c) Final chemical phosphorylation

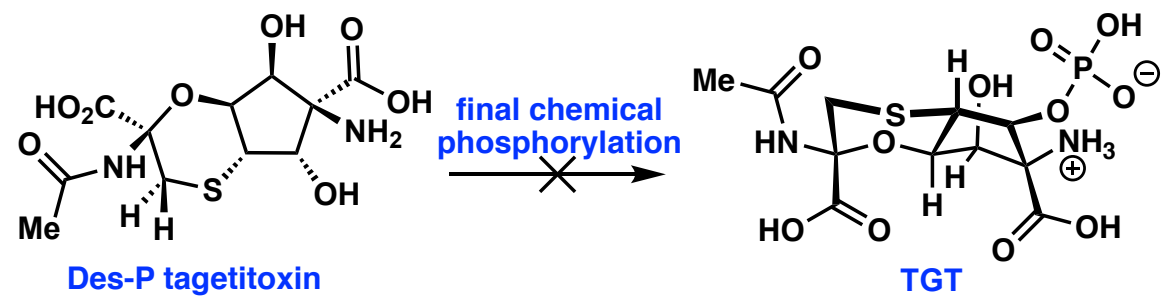

- $\mathrm{SM}$ is insoluble in $\mathrm{MeCN}$, acetone, $\mathrm{CH}_{2} \mathrm{Cl}_{2}$, $\mathrm{MeOH}, \mathrm{DMF}, \mathrm{THF}$, ethyl acetate...milky suspension soluble in DMSO and pyridine.

- ${ }^{1} \mathrm{H}$ NMR in $\mathrm{D}_{2} \mathrm{O}$ is good, but not clear in DMSO-d $\mathrm{d}_{6}$ or pyridine- $\mathrm{d}_{5}$.

\begin{tabular}{|c|c|}
\hline Conditions & Results \\
\hline $\begin{aligned} & \text { - } \mathrm{Pr}_{2} \mathrm{NP}(\mathrm{OBn})_{2} \text {, tetrazole, MeCN, rt } \\
& \text { - } \mathrm{Pr}_{2} \mathrm{NP}(\mathrm{OBn})_{2} \text {, tetrazole, MeCN/pyridine (1:1), rt } \\
& \text { - } \mathrm{Bu}_{4} \mathrm{~N}^{+} \mathrm{H}_{2} \mathrm{PO}_{4}^{-}, \mathrm{Cl}_{3} \mathrm{CCN}, \mathrm{MeCN}, \mathrm{rt} \\
& \text { - } \mathrm{Bu}_{4} \mathrm{~N}^{+} \mathrm{H}_{2} \mathrm{PO}_{4}^{-}, \mathrm{Cl}_{3} \mathrm{CCN}, \mathrm{DMSO}, \mathrm{rt} \\
& \text { - } \mathrm{Bu}_{4} \mathrm{~N}^{+} \mathrm{H}_{2} \mathrm{PO}_{4}^{-}, \mathrm{Cl}_{3} \mathrm{CCN}, \text { pyridine, rt } \\
& \text { - } \text { sodium } 3-(\text { trimethylsilyl)-1- propanesulfonate, } \\
& \text { } \mathrm{HOP}(\mathrm{O})\left(\mathrm{NH}_{2}\right)_{2}, \mathrm{PBS} \text { buffer(pH 6-7), rt } \\
& \text { - } \mathrm{POCl}{ }_{3}, \text { pyridine, rt } \\
& \text { - } \mathrm{PO}(\mathrm{OPh})_{2} \mathrm{Cl}, \text { pyridine, rt }\end{aligned}$ & $\begin{array}{l}\text { no reaction } \\
\text { no reaction } \\
\text { no reaction } \\
\text { inconclusive } \\
\text { no reaction } \\
\text { no reaction } \\
\text { decomposition } \\
\text { no reaction }\end{array}$ \\
\hline
\end{tabular}

Lack of solubility in organic solvents rendered des-P tagetitoxin a very difficult intermediate to handle, which led us to installation of $P(V)$ before global deprotection.

Scheme S18. Direct phosphorylation on des-P tagetitoxin 


\section{IV) Summary of Third-Generation Synthesis Optimization}

Third-generation modification: achieved phosphorylation at an earlier stage

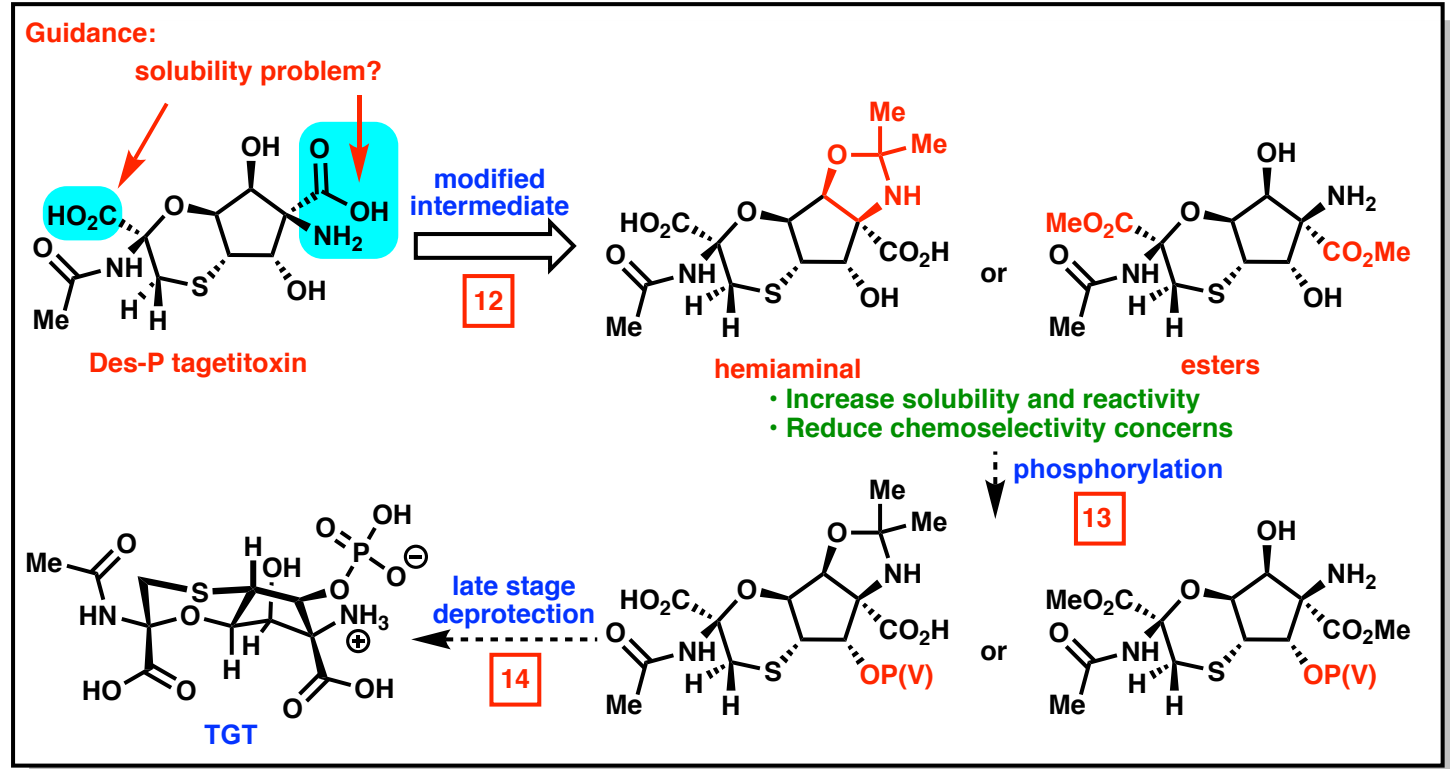

12 - Modification of phosphorylation precursor

- Hemiaminal protection

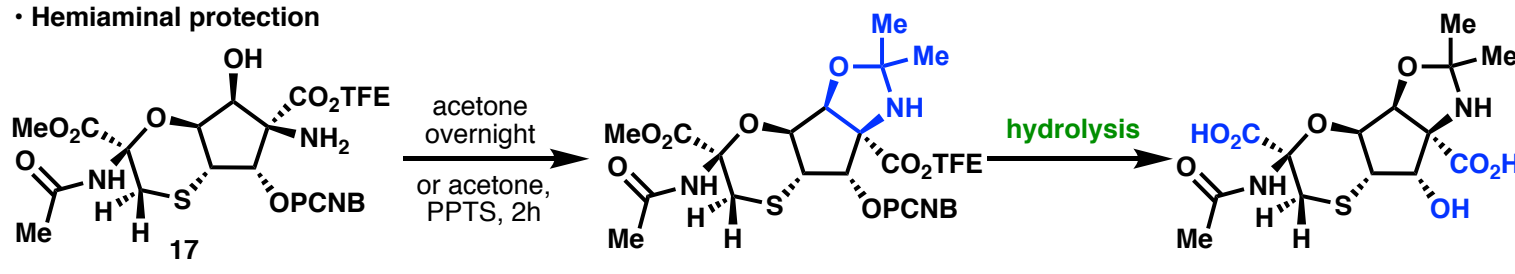

\begin{tabular}{ll}
\hline Hydrolysis conditions & Results \\
\hline$\cdot \mathrm{MeOH}-\mathrm{d}_{4}, \mathrm{Ba}(\mathrm{OH})_{2}(0.1 \mathrm{M} \mathrm{MeOD})$ & decomposition \\
$\cdot-\mathrm{MeOH}-\mathrm{d}_{4}, \mathrm{Ba}(\mathrm{OH})_{2}\left(0.1 \mathrm{M} \mathrm{H}_{2} \mathrm{O}\right)$ & messy, minor desired product \\
$\cdot \mathrm{CD}_{3} \mathrm{CN}, \mathrm{Ba}(\mathrm{OH})_{2}\left(0.1 \mathrm{M} \mathrm{H}_{2} \mathrm{O}\right)$ & no reaction, $\mathrm{Ba}(\mathrm{OH})_{2}$ insoluble \\
$\cdot-\mathrm{Ba}(\mathrm{OH})_{2}\left(0.1 \mathrm{M} \mathrm{D}_{2} \mathrm{O}\right)$, & \\
$\mathrm{D}_{2} \mathrm{O} / \mathrm{CH}_{3} \mathrm{CN}(10: 1), 70 \mathrm{~min}$ & good, clean crude HNMR \\
\hline
\end{tabular}

- Esters

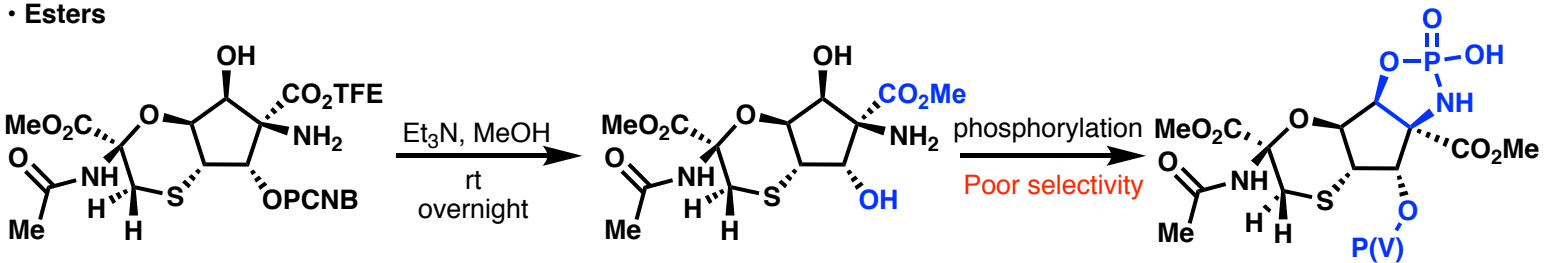

- Hemiaminal \& Esters

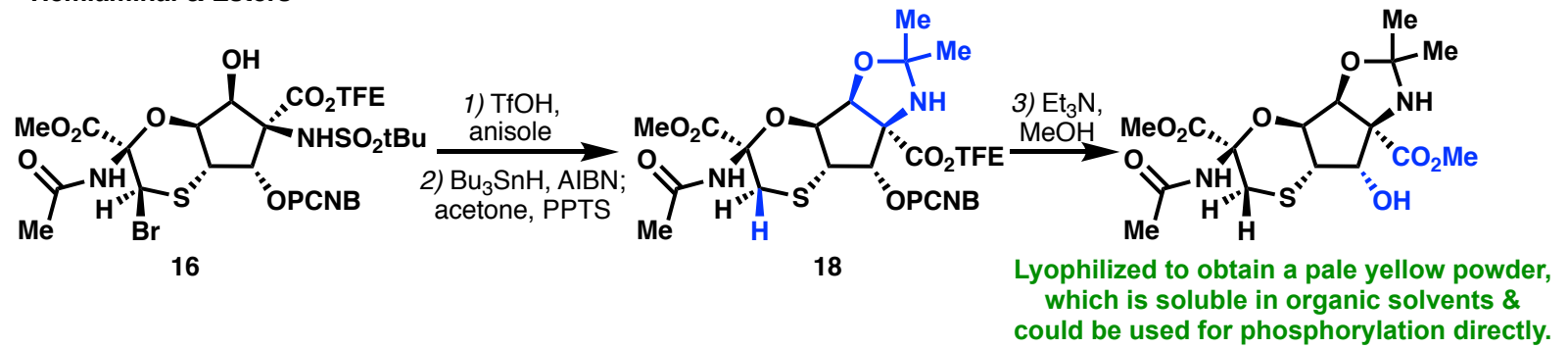

Scheme S19. Construction of phosphorylation product 
13 - Optimization of phosphorylation

a) $\mathrm{P}(\mathrm{III})$ to $\mathrm{P}(\mathrm{V})$ strategy: Oxidation was difficult to control. $S$ tends to get over oxidized.

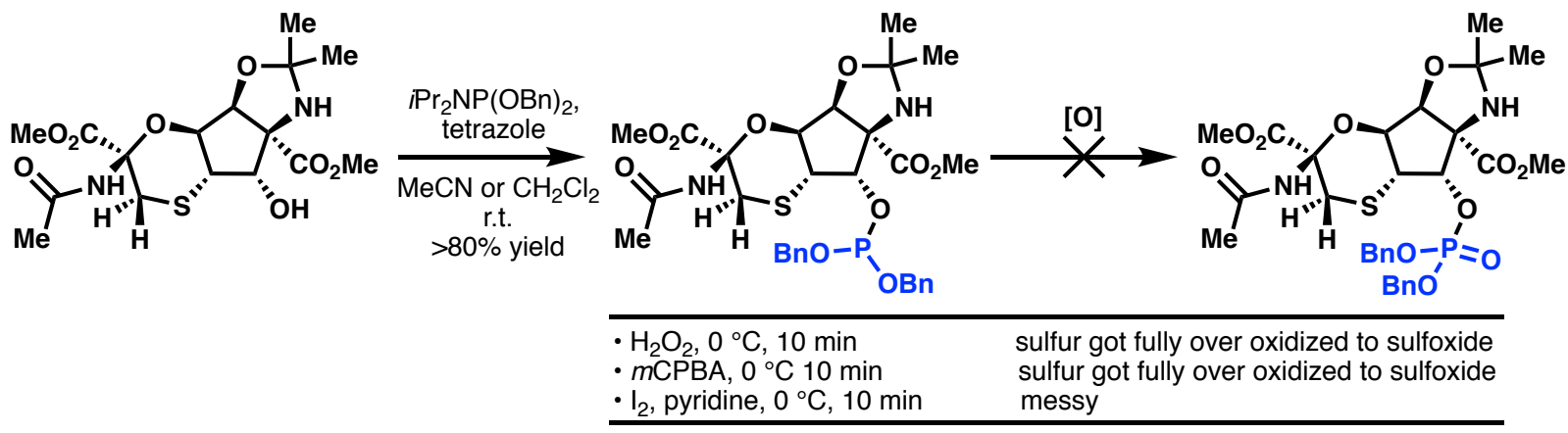

b) Conventional $\mathrm{P}(\mathrm{V})$ strategy: conditions were either too harsh or invalid.

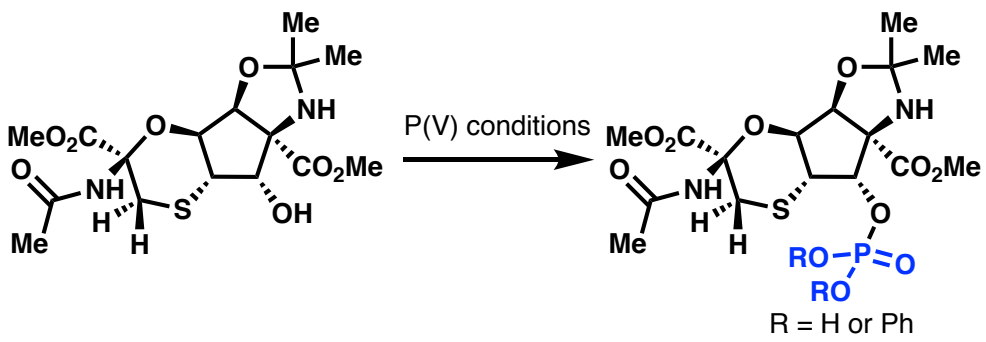

- $\mathrm{POCl}_{3}, \mathrm{Et}_{3} \mathrm{~N}, \mathrm{THF}, 0^{\circ} \mathrm{C} ; \mathrm{H}_{2} \mathrm{O}$

- $\mathrm{Bu}_{4} \mathrm{~N}^{+} \mathrm{H}_{2} \mathrm{PO}_{4}^{-}, \mathrm{Cl}_{3} \mathrm{CCN}, \mathrm{CH}_{3} \mathrm{CN}, \mathrm{rt}$

- $(\mathrm{PhO})_{2} \mathrm{POCl}, \mathrm{Pyr}$., $\mathrm{CH}_{2} \mathrm{Cl}_{2}$, rt

decomposition decomposition

- (PhO) ${ }_{2} \mathrm{POCl}$, tBuOLi, THF, -78 ${ }^{\circ} \mathrm{C}$ - rt no reaction

full conversion of SM, trace of product

- $(\mathrm{PhO})_{2} \mathrm{POCl}, \mathrm{Et}_{3} \mathrm{~N}, \mathrm{TiCl}_{4}$ (cat.), THF, rt no reaction

c) Final phosphorylation with (+)- $\Psi$ :

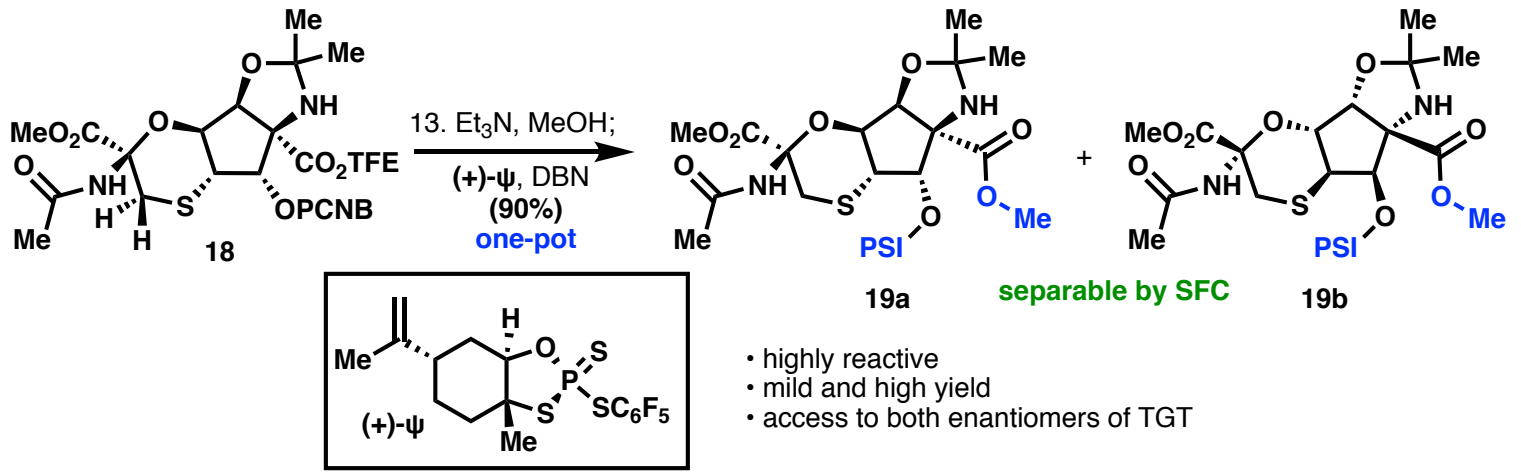

Scheme S20. Optimization of phosphorylation 
14 - Optimization of final deprotection
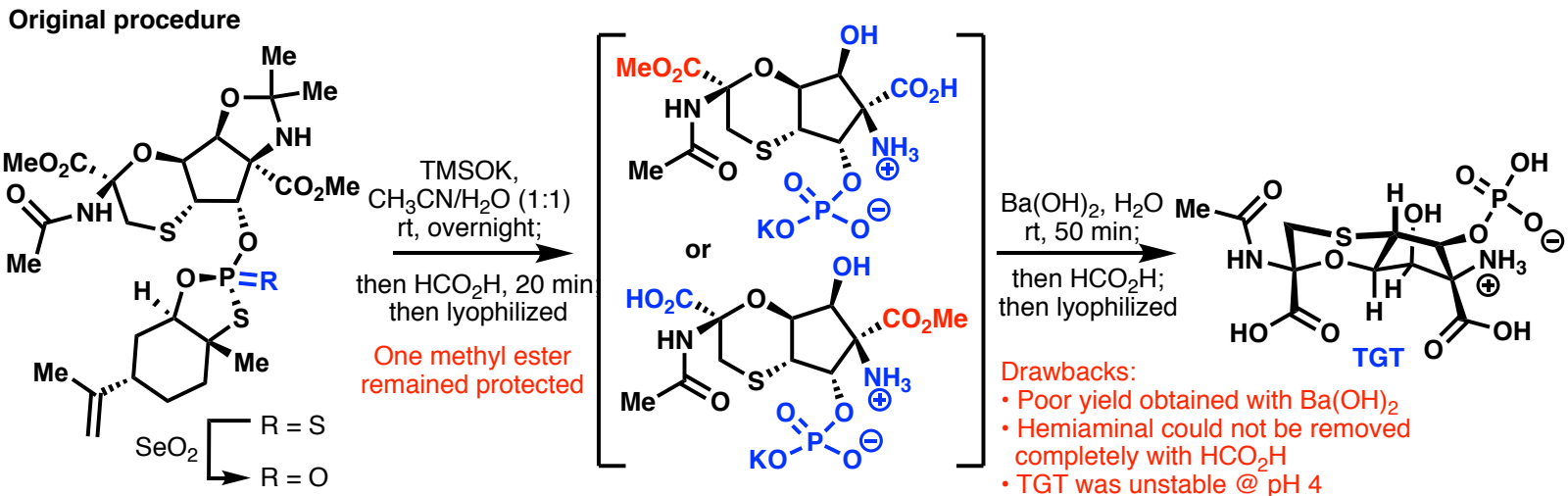

Optimized procedure

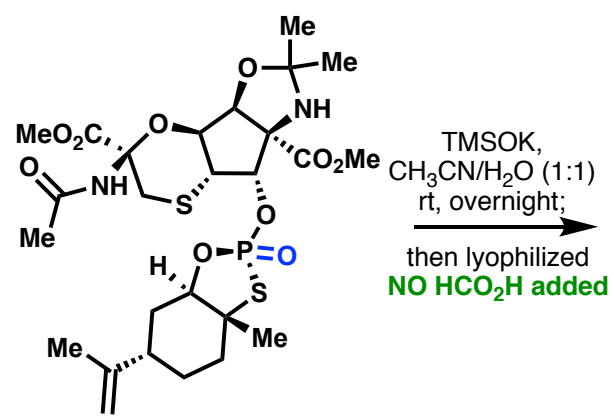

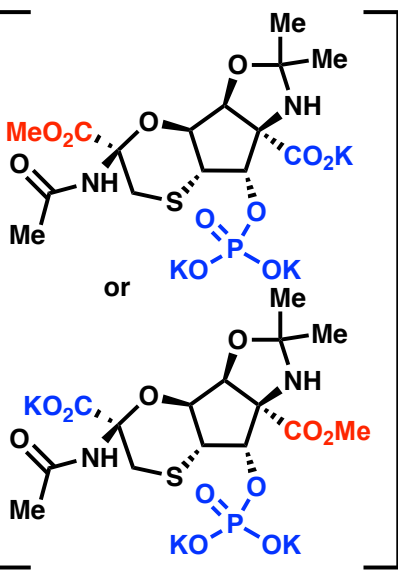

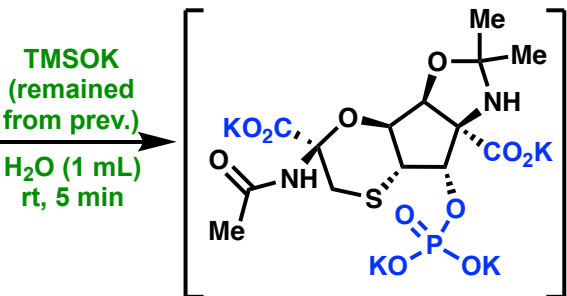

$\mathrm{MeONH}_{2} \cdot \mathrm{HCl}, 30 \mathrm{~min}$ then $\mathrm{NaHCO}_{3}$ (solid) then DEAE purification

- The second ester was hydrolized by changing solvent to pure $\mathrm{H}_{2} \mathrm{O}$

- Hemiaminal was removed quickly and thoroughly with $\mathrm{MeONH}_{2}$

-TGT was stable @ pH 8 (adjusted with solid $\mathrm{NaHCO}_{3}$ )

- High yield Easily handled in one-pot

Scheme S21. Optimization of final deprotection 


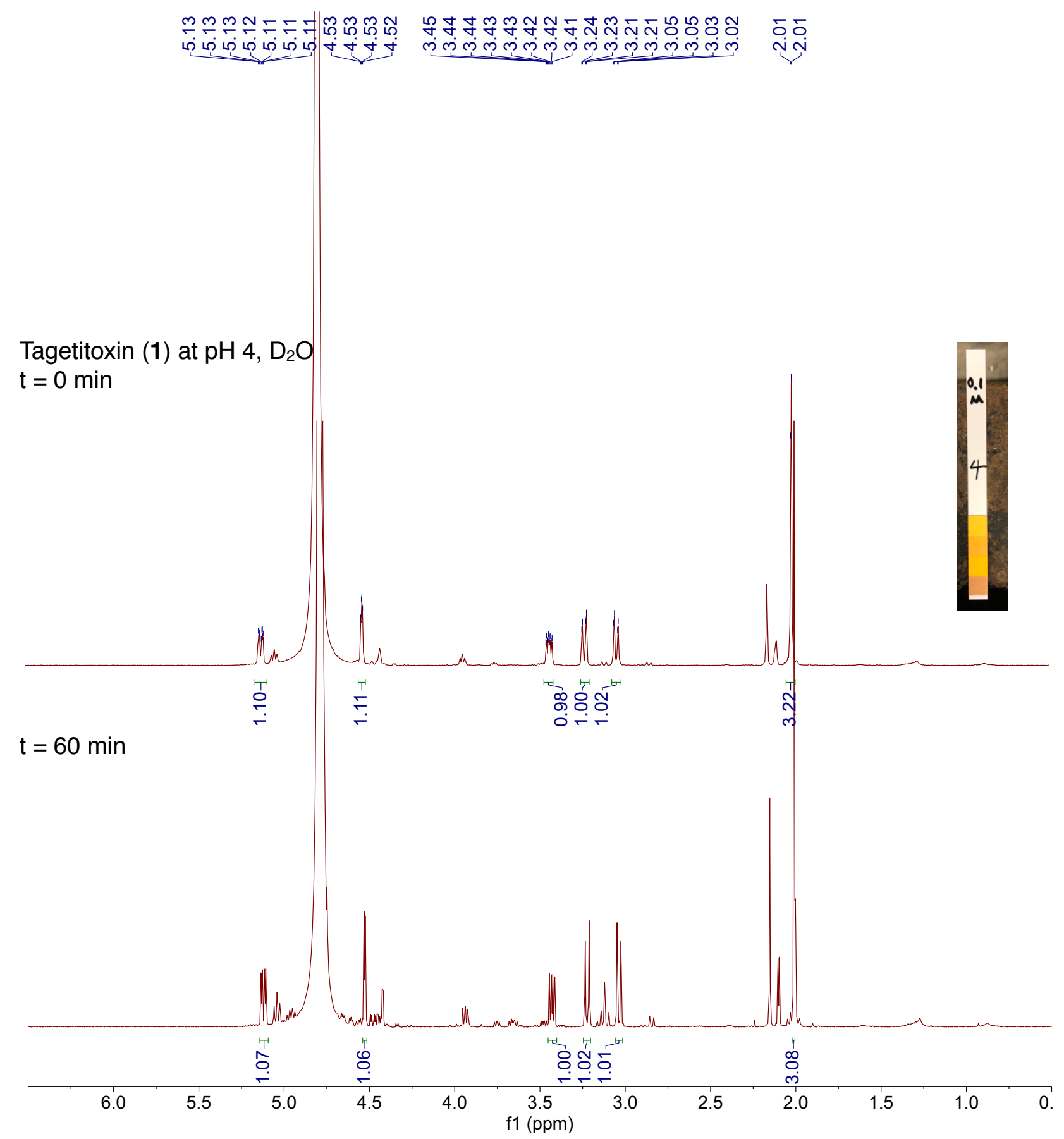

Figure S1. Stacked ${ }^{1} \mathrm{H}-\mathrm{NMR}$ spectra demonstrating instability of $\mathbf{1}$ to mildly acidic conditions 


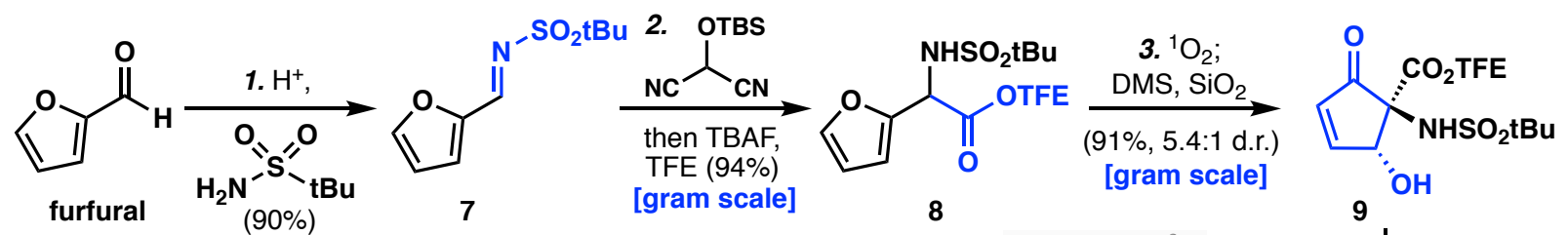
[gram scale]

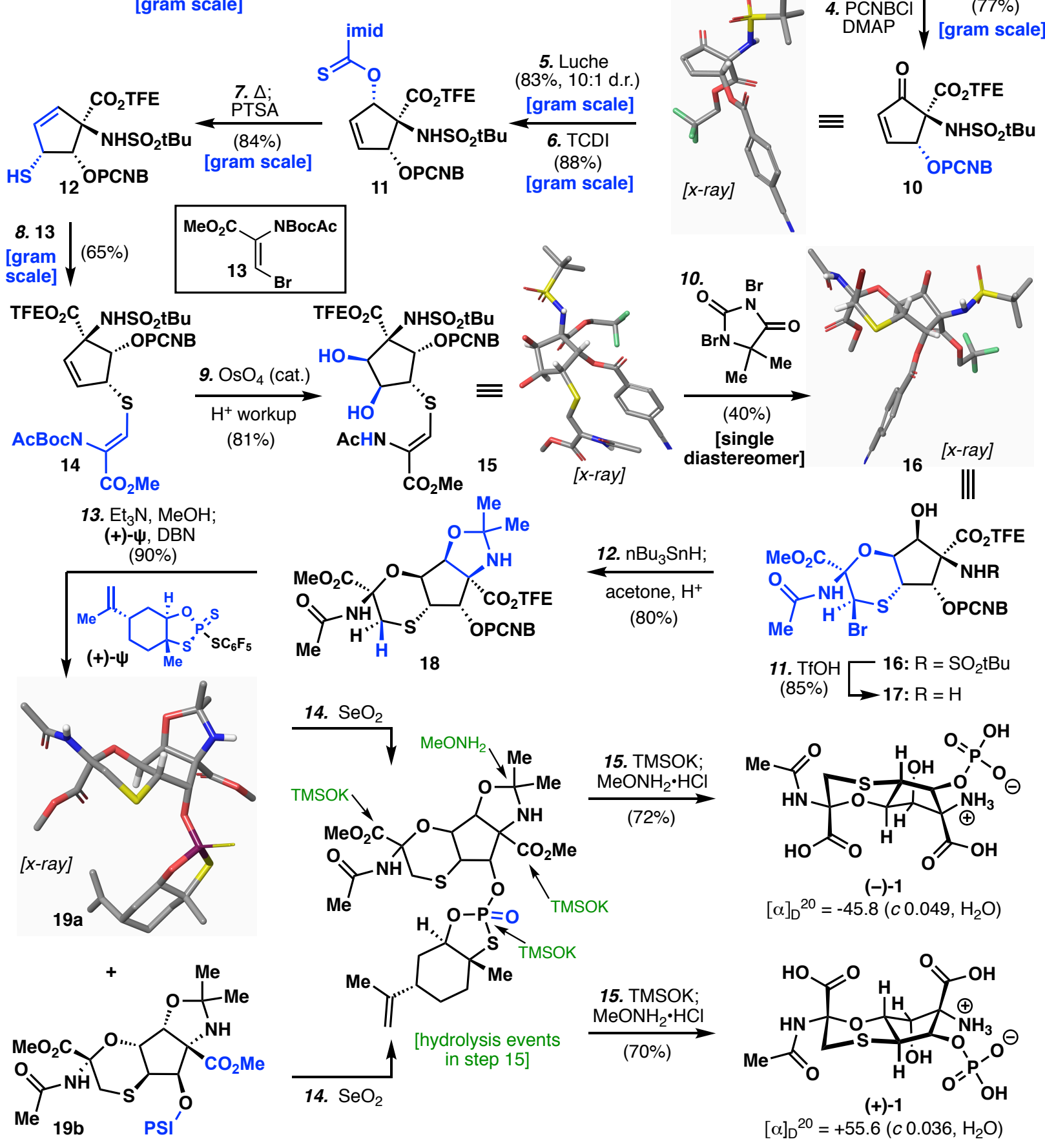

${ }^{a}$ Reagents and conditions: (1) tert-butylsulfonamide ( $\left.0.67 \mathrm{eq}\right)$, Amberlyst $15(10 \mathrm{mg} / \mathrm{mmol})$, toluene, reflux, $48 \mathrm{~h}$ ( $90 \%$ yield). (2) MAC reagent (1.2 eq), $\mathrm{Et}_{3} \mathrm{~N}(1.5 \mathrm{eq})$, toluene, rt, $20 \mathrm{~min}$; $\operatorname{TFE}(10 \mathrm{eq}), \operatorname{TBAF}(1.5 \mathrm{eq}),-45^{\circ} \mathrm{C}, 30 \mathrm{~min}(94 \%$ yield). (3) methylene blue (cat.), dry $\mathrm{O}_{2}$, sunlight lamp ( $\left.150 \mathrm{~W}\right), \mathrm{CH}_{2} \mathrm{Cl}_{2},-78^{\circ} \mathrm{C}, 2 \mathrm{~h}$; DMS (5 eq), $\mathrm{SiO}_{2}$, (91\% yield, 5.4:1 d.r.). (4) PCNBCl (1.2 eq), DMAP (1.2 eq), $\mathrm{CH}_{2} \mathrm{Cl}_{2},-78^{\circ} \mathrm{C}, 30 \mathrm{~min}$ ( $77 \%$ yield, 5.4:1 d.r.). (5) $\mathrm{CeCl}_{3} \cdot 7 \mathrm{H}_{2} \mathrm{O}(6 \mathrm{eq}), \mathrm{NaBH}_{4}(6 \mathrm{eq}), \mathrm{MeOH},-60{ }^{\circ} \mathrm{C}, 30 \mathrm{~min}(83 \%$ yield, 10:1 d.r.). (6) TCDI ( 2 eq), $\mathrm{CH}_{3} \mathrm{CN}$, rt, overnight ( $88 \%$ yield). (7) $\mathrm{BHT}\left(0.05\right.$ eq), toluene, $115^{\circ} \mathrm{C}, 22 \mathrm{~h}$; PTSA (2.2 eq), acetone $/ \mathrm{H}_{2} \mathrm{O}$ (1:1), rt, $2 \mathrm{~h}$ (84\% yield). (8) 13 (1.2 eq), $\mathrm{Et}_{3} \mathrm{~N}$ (2 eq), $\mathrm{CH}_{3} \mathrm{CN}$, rt, $30 \mathrm{~min}$ (65\% yield). (9) $\mathrm{OsO}_{4}(0.1 \mathrm{eq}), \mathrm{NMO}(5 \mathrm{eq})$, 
citric acid (5 eq), $\mathrm{tBuOH} /$ acetone $/ \mathrm{H}_{2} \mathrm{O}(1: 1: 1), 35^{\circ} \mathrm{C}, 36 \mathrm{~h}$; conc. $\mathrm{HCl}, \mathrm{rt}, 1 \mathrm{~h}$ (81\% yield). (10) DBDMH (1.25 eq), AcOH (1.25 eq), $\mathrm{CHCl}_{3}(0.05 \mathrm{M}), \mathrm{rt}, 5 \mathrm{~min} ; 50{ }^{\circ} \mathrm{C}(0.015 \mathrm{M}), 30 \mathrm{~min}(40 \%$ yield $) . \quad$ (11) anisole $(50 \mathrm{eq}), \mathrm{TfOH}(10 \mathrm{eq}), \mathrm{CH}_{2} \mathrm{Cl}_{2}, 0{ }^{\circ} \mathrm{C}, 20 \mathrm{~min}$ (85\% yield). (12) $\mathrm{nBu}_{3} \mathrm{SnH}(5 \mathrm{eq})$, $\mathrm{AIBN}(0.1 \mathrm{eq})$, benzene, $85^{\circ} \mathrm{C}, 40 \mathrm{~min}$; PPTS (0.1 eq), acetone, rt, $3 \mathrm{~h}(80 \%$ yield). (13) Et $3 \mathrm{~N}$ (100 eq), $\mathrm{CH}_{3} \mathrm{OH}$, rt, overnight; (+)- $\Psi$ (2 eq), DBN (3 eq), $\mathrm{CH}_{3} \mathrm{CN}, \mathrm{rt}, 20$ min (90\% yield, 1:1 d.r.). (14) $\mathrm{SeO}_{2}(15 \mathrm{eq}), \mathrm{CH}_{3} \mathrm{CN} 0$ ${ }^{\circ} \mathrm{C}, 3$ min. (15) TMSOK (5 eq), $\mathrm{CH}_{3} \mathrm{CN} / \mathrm{H}_{2} \mathrm{O}(1: 1)$, rt, overnight; $\mathrm{MeONH}_{2} \bullet \mathrm{HCl}$ (5 eq), $\mathrm{H}_{2} \mathrm{O}$, rt, 30 min (72\% yield for (-)-1, 70\% yield for $(+)-1)$. MAC reagent=2-(tert-butyldimethylsilanyloxy)malononitrile; $\mathrm{rt}=$ room temperature; TFE $=2,2,2$-trifluoroethanol; TBAF $=$ tetra- $n$-butylammonium fluoride; DMS $=$ dimethyl sulfide; $\mathrm{PCNBCl}=p$-cyanobenzoyl chloride; DMAP $=4$ dimethylaminopyridine; TCDI = 1,1'-thiocarbonyldiimidazole; $\mathrm{BHT}=$ dibutylhydroxytoluene; PTSA $=p$-toluenesulfonic acid; $\mathrm{NMO}=N$-methylmorpholine $N$-oxide; $\mathrm{DBDMH}=1,3$-dibromo-5,5-dimethylhydantoin; $\mathrm{AcOH}=$ acetic acid $\mathrm{TfOH}=$ trifluoromethanesulfonic acid; AIBN = 2,2'-azobis(2-methylpropionitrile); PPTS = pyridinium $p$-toluenesulfonate; DBN = 1,5diazabicyclo(4.3.0)non-5-ene; TMSOK = potassium trimethylsilanolate.

Scheme S22. The overview of final route 


\section{V) Experimental Procedures and Characterization Data}

\section{Compound 7}<smiles>CCCOS(=O)(=N/C=C/c1ccco1)OC(C)(C)C</smiles>

Experimental: To a solution of tert-butanesulfonamide $(6.85 \mathrm{~g}, 50 \mathrm{mmol})$ and furfural $(6.2 \mathrm{~mL}, 75$ mmol, 1.5 equiv) in toluene ( $150 \mathrm{~mL})$ was added Amberlyst 15 (500 mg, $10 \mathrm{mg} / \mathrm{mmol})$. A DeanStark apparatus was affixed and the mixture was heated to reflux under argon atmosphere for 48 $\mathrm{h}$. The mixture was filtered through a pad of Celite and rinsed forward with EtOAc. The filtrate was concentrated via rotary evaporation. The residue was purified via column chromatography $\left(\mathrm{SiO}_{2}\right.$, EtOAc/hexanes $=1: 4)$ to give product 7 as pale yellow solid $(9.64 \mathrm{~g}, 45 \mathrm{mmol})$ in $90 \%$ yield. Spectroscopic data matched reported literature data. ${ }^{[4]}$

Physical State: pale yellow solid;

TLC: $R_{f}=0.4($ EtOAc/hexanes = 1:2);

${ }^{1} \mathrm{H}$ NMR $\left(600 \mathrm{MHz}, \mathrm{CDCl}_{3}\right) \delta 8.78(\mathrm{~s}, 1 \mathrm{H}), 7.78(\mathrm{dd}, J=1.7,0.8 \mathrm{~Hz}, 1 \mathrm{H}), 7.35(\mathrm{dd}, J=3.7,0.8 \mathrm{~Hz}$, $1 \mathrm{H}), 6.67$ (dd, J=3.6, 1.7 Hz, 1H), $1.48(\mathrm{~s}, 9 \mathrm{H})$;

${ }^{13} \mathrm{C} \mathrm{NMR}\left(150 \mathrm{MHz}, \mathrm{CDCl}_{3}\right) \delta 158.4,149.7,149.5,124.4,113.8,58.6,24.2(3 \mathrm{C}) ;$

HRMS (m/z): calculated for $\mathrm{C}_{9} \mathrm{H}_{13} \mathrm{NO}_{3} \mathrm{~S}[\mathrm{M}+\mathrm{H}]+216.0689$, found 216.0693 .

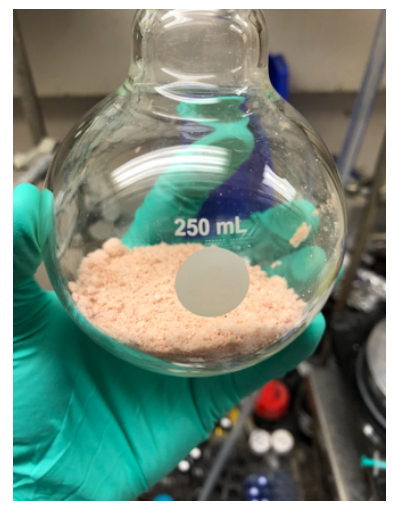

over $30 \mathrm{~g}$ prepared 


\section{Compound 8}<smiles>CCOC(=O)C(Nc1ccco1)C(=O)OCC</smiles>

Experimental: To a solution of $7(5.30 \mathrm{~g}, 24.6 \mathrm{mmol})$ and MAC reagent [5] $(5.80 \mathrm{~g}, 29.6 \mathrm{mmol}$, 1.2 equiv) in toluene $(150 \mathrm{~mL}, 0.16 \mathrm{M})$ was added $\mathrm{Et}_{3} \mathrm{~N}\left(5.11 \mathrm{~mL}, 37.0 \mathrm{mmol}, 1.5\right.$ equiv) at $0^{\circ} \mathrm{C}$. The reaction mixture was stirred at $\mathrm{rt}$ for $20 \mathrm{~min}$. The reaction was then cooled to $-45^{\circ} \mathrm{C}$ and TFE (18.0 mL, $246 \mathrm{mmol}, 10$ equiv) was added in one portion. TBAF (1.0 M in THF, $37.0 \mathrm{~mL}, 37.0$ mmol, 1.5 equiv) was added dropwise. The mixture was stirred at $-45^{\circ} \mathrm{C}$ for 30 min, then quenched with $\mathrm{NH}_{4} \mathrm{Cl}$ (sat. aq. $200 \mathrm{~mL}$ ) and extracted with EtOAc (200 mL x 3). The combined organic layers were washed with brine $(300 \mathrm{~mL})$, dried over $\mathrm{Na}_{2} \mathrm{SO}_{4}$, then concentrated via rotary evaporation to give a pale yellow oil. The crude product was purified via column chromatography $\left(\mathrm{SiO}_{2}\right.$, EtOAc/hexanes = 1:4) to give product $8(7.97 \mathrm{~g}, 23.2 \mathrm{mmol})$ as pale yellow solid in $94 \%$ yield.

NOTE: The aqueous waste contains toxic cyanide ion. It should be treated with excess bleach.

Physical State: pale yellow solid;

Melting Point: $76-78^{\circ} \mathrm{C}$;

TLC: $R_{f}=0.6($ EtOAc/hexanes = 1:2);

1H NMR $\left(600 \mathrm{MHz}, \mathrm{CDCl}_{3}\right) \delta 7.40(\mathrm{dd}, J=1.8,0.9 \mathrm{~Hz}, 1 \mathrm{H}), 6.39(\mathrm{~d}, J=3.4 \mathrm{~Hz}, 1 \mathrm{H}), 6.37$ (dd, $J$ $=3.3,1.9 \mathrm{~Hz}, 1 \mathrm{H}), 5.37(\mathrm{~d}, J=9.4 \mathrm{~Hz}, 1 \mathrm{H}), 5.11(\mathrm{~d}, J=9.4 \mathrm{~Hz}, 1 \mathrm{H}), 4.64-4.57(\mathrm{~m}, 1 \mathrm{H}), 4.57-$ $4.50(\mathrm{~m}, 1 \mathrm{H}), 1.36(\mathrm{~s}, 9 \mathrm{H})$;

${ }^{13} \mathrm{C} \mathrm{NMR}\left(150 \mathrm{MHz}, \mathrm{CDCl}_{3}\right) \delta 168.1,147.6,143.6,122.5\left(\mathrm{q}, J_{C-F}=277.5 \mathrm{~Hz}\right), 111.0,109.5,61.6$ (q, $\left.J_{C-F}=37.3 \mathrm{~Hz}\right), 60.6,54.5,24.0(3 \mathrm{C})$;

${ }^{19}$ F NMR (376 MHz, $\left.\mathrm{CDCl}_{3}\right) \delta-74.0$;

HRMS (m/z): calculated for $\mathrm{C}_{12} \mathrm{H}_{16} \mathrm{~F}_{3} \mathrm{NO}_{5} \mathrm{~S}[\mathrm{M}+\mathrm{H}]^{+} 344.0774$, found 344.0782 . 


\section{Compound 9 and 9'}<smiles>CCOC(=O)NC1(COC(=O)OCC)C(=O)C=C[C@H]1O</smiles>

9<smiles>CCOC(=O)NC1(OC(=O)OCC)C(=O)C=CC1O</smiles>

9'

d.r. 5.4:1 mixture

Experimental: To a solution of $8(4.2 \mathrm{~g}, 12.2 \mathrm{mmol})$ in $\mathrm{CH}_{2} \mathrm{Cl}_{2}(100 \mathrm{~mL}, 0.12 \mathrm{M})$ was added methylene blue $(2 \mathrm{mg})$. The mixture was stirred at $\mathrm{rt}$ for $5 \mathrm{~min}$ to allow for full dissolution of methylene blue, then cooled to $-78{ }^{\circ} \mathrm{C}$. The transparent blue reaction mixture was sparged continuously with dry $\mathrm{O}_{2}$ and irradiated under a sunlight lamp (150 W). Upon completion, (approximately $2 \mathrm{~h}$, monitored by TLC), $\mathrm{Me}_{2} \mathrm{~S}(4.45 \mathrm{ml}, 61.0 \mathrm{mmol}, 5.0$ equiv) was added to quench the reaction. After $10 \mathrm{~min}$, silica gel (approximately $20 \mathrm{~g}$ ) was added at $-78^{\circ} \mathrm{C}$. The mixture was warmed to rt, then the solvent was removed via rotary evaporation. The residual dry powder was flushed with EtOAc $(200 \mathrm{~mL})$ and the resulting solution was concentrated via rotary evaporation to give a brown oil. The crude product was purified via column chromatography $\left(\mathrm{SiO}_{2}\right.$, EtOAc/hexanes $=1: 5)$ to give a mixture of two inseparable diastereomers 9 and $\mathbf{9}^{\prime}$ (3.99 g, 11.1 $\mathrm{mmol}$, dr 5.4:1) as white foam in $91 \%$ yield.

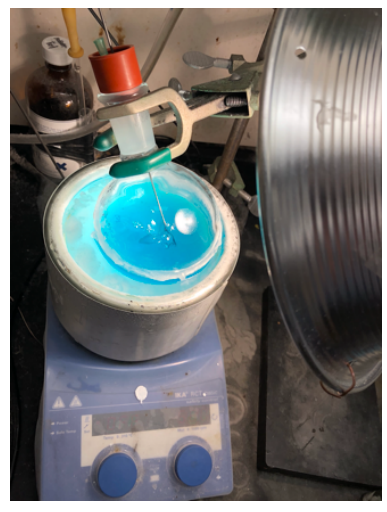

a) singlet ${ }^{1} \mathrm{O}_{2}$ reaction

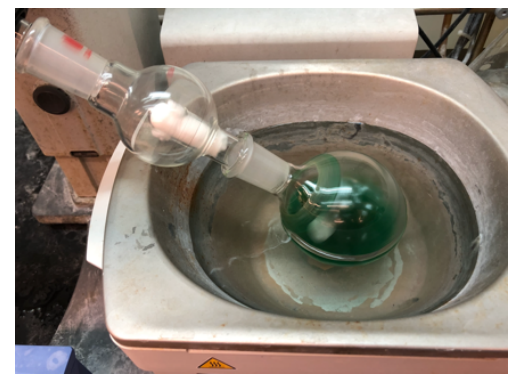

b) removing solvent

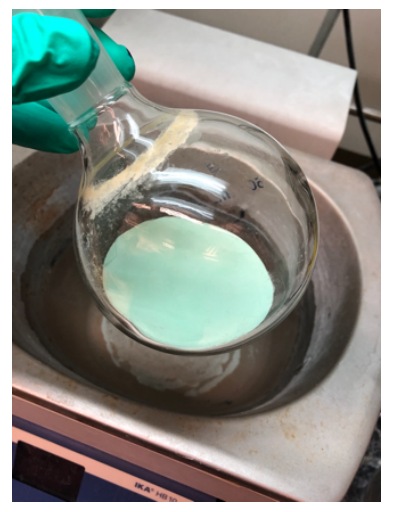

c) dried residue

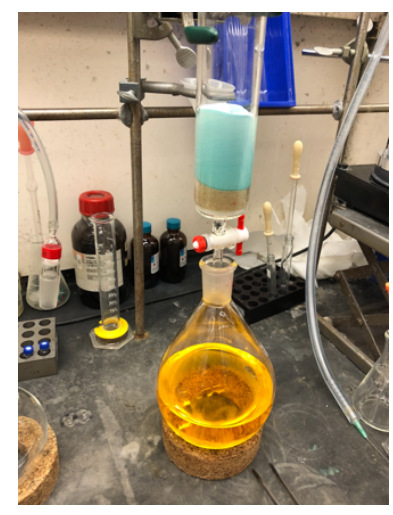

d) flushed with EtOAc 
Physical State: white foam;

TLC: $R_{f}=0.5($ EtOAc/hexanes = 1:2);

${ }^{1}$ H NMR $\left(600 \mathrm{MHz}, \mathrm{CDCl}_{3}\right) \delta 7.74(\mathrm{dd}, J=6.1,2.9 \mathrm{~Hz}, 0.18 \mathrm{H}), 7.69(\mathrm{dd}, J=6.0,2.1 \mathrm{~Hz}, 1 \mathrm{H}), 6.44$ (dd, $J=6.0,1.9 \mathrm{~Hz}, 1 \mathrm{H}), 6.41(\mathrm{~d}, J=6.1 \mathrm{~Hz}, 0.18 \mathrm{H}), 5.27(\mathrm{q}, J=2.3 \mathrm{~Hz}, 1 \mathrm{H}), 5.22(\mathrm{dd}, J=8.3$, $2.9 \mathrm{~Hz}, 0.18 \mathrm{H}), 5.17(\mathrm{~s}, 0.18 \mathrm{H}), 5.09(\mathrm{~s}, 1 \mathrm{H}), 4.69-4.60(\mathrm{~m}, 0.18 \mathrm{H}), 4.57-4.46(\mathrm{~m}, 2 \mathrm{H}), 4.43-$ $4.36(\mathrm{~m}, 0.18 \mathrm{H}), 3.69(\mathrm{~d}, J=5.6 \mathrm{~Hz}, 1 \mathrm{H}), 3.61(\mathrm{~d}, J=8.6 \mathrm{~Hz}, 0.18 \mathrm{H}), 1.47(\mathrm{~s}, 1.62 \mathrm{H}), 1.44(\mathrm{~s}$, $9 \mathrm{H})$;

${ }^{13} \mathrm{C}$ NMR $\left(150 \mathrm{MHz}, \mathrm{CDCl}_{3}\right) \delta 196.1,195.8,166.3,165.6,162.3,161.4,133.1,132.7,122.4$ (q, $\left.J_{C-F}=277.4 \mathrm{~Hz}\right), 78.6,75.5,74.7,71.2,62.0\left(\mathrm{q}, J_{C-F}=37.4 \mathrm{~Hz}\right), 61.9\left(\mathrm{q}, J_{C-F}=37.5 \mathrm{~Hz}\right), 61.6$, 61.0, $24.4(3 \mathrm{C}), 24.3(3 \mathrm{C})$;

Note: The $\mathrm{CF}_{3}$ carbon of the minor diastereomer could not be found.

${ }^{19} \mathrm{~F} \mathrm{NMR}\left(376 \mathrm{MHz}, \mathrm{CDCl}_{3}\right) \delta-73.84$ (overlapped);

HRMS (m/z): calculated for $\mathrm{C}_{12} \mathrm{H}_{16} \mathrm{~F}_{3} \mathrm{NO}_{6} \mathrm{~S}[\mathrm{M}+\mathrm{Na}]^{+}$382.0543, found 382.0541. 


\section{Compound 10 and 10'}

Experimental: To a solution of 4-Cyanobenzoyl chloride ( $2.37 \mathrm{~g}, 14.4 \mathrm{mmol}, 1.2$ equiv) in $\mathrm{CH}_{2} \mathrm{Cl}_{2}$ $(20 \mathrm{~mL})$ was added DMAP $\left(1.75 \mathrm{~g}, 14.4 \mathrm{mmol}, 1.2\right.$ equiv) at $0{ }^{\circ} \mathrm{C}$. The mixture was warmed to rt and stirred for $2 \mathrm{~min}$. The mixture was then added to a solution (precooled to $-78^{\circ} \mathrm{C}$ ) of alcohol 9 and 9' $(4.3 \mathrm{~g}, 12.0 \mathrm{mmol})$ in $\mathrm{CH}_{2} \mathrm{Cl}_{2}(50 \mathrm{~mL})$, dropwise. After $30 \mathrm{~min}$ at $-78{ }^{\circ} \mathrm{C}$, the reaction was quenched with $\mathrm{NaHCO}_{3}$ (sat. aq., $100 \mathrm{~mL}$ ). The mixture was extracted with EtOAc (100 mL x 3), dried over $\mathrm{Na}_{2} \mathrm{SO}_{4}$, then concentrated via rotary evaporation. The crude product was purified via column chromatography $\left(\mathrm{SiO}_{2}\right.$, EtOAc:hexanes $\left.=1: 3\right)$ to give product $10(3.80 \mathrm{~g}, 7.78 \mathrm{mmol})$ as white solid and 10' (0.70 g, $1.44 \mathrm{mmol}$ ) as white foam in $77 \%$ yield.

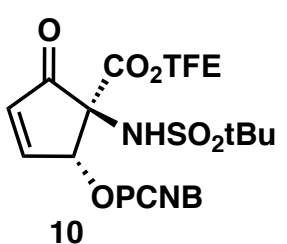

Physical State: white solid;

Melting Point: $182-184^{\circ} \mathrm{C}$;

TLC: $R_{f}=0.4(E t O A c / h e x a n e s=1: 2)$;

${ }^{1} \mathrm{H} \mathrm{NMR}\left(600 \mathrm{MHz}, \mathrm{CDCl}_{3}\right) \delta 8.05(\mathrm{~d}, J=8.6 \mathrm{~Hz}, 2 \mathrm{H}), 7.76(\mathrm{~d}, J=8.5 \mathrm{~Hz}, 2 \mathrm{H})$, $7.64(\mathrm{dd}, J=6.2,2.2 \mathrm{~Hz}, 1 \mathrm{H}), 6.63(\mathrm{dd}, J=6.2,1.6 \mathrm{~Hz}, 1 \mathrm{H}), 6.53(\mathrm{t}, J=1.9$ $\mathrm{Hz}, 1 \mathrm{H}), 5.24(\mathrm{~s}, 1 \mathrm{H}), 4.66-4.58(\mathrm{~m}, 1 \mathrm{H}), 4.44-4.35(\mathrm{~m}, 1 \mathrm{H}), 1.44(\mathrm{~s}, 9 \mathrm{H})$;

${ }^{13} \mathrm{C}$ NMR $\left(150 \mathrm{MHz}, \mathrm{CDCl}_{3}\right) \delta 193.9,164.5,164.2,155.9,135.7,132.5$ (2C), 132.2, 130.4 (2C), $122.2\left(q, J_{C-F}=277.3 \mathrm{~Hz}\right), 117.7,117.5,80.6,72.1,62.2\left(q, J_{C-F}=37.7 \mathrm{~Hz}\right), 61.2,24.1(3 C)$;

${ }^{19} \mathrm{~F}$ NMR $\left(376 \mathrm{MHz}, \mathrm{CDCl}_{3}\right) \delta-73.7$;

HRMS (m/z): calculated for $\mathrm{C}_{20} \mathrm{H}_{19} \mathrm{~F}_{3} \mathrm{~N}_{2} \mathrm{O}_{7} \mathrm{~S}[\mathrm{M}+\mathrm{Na}]^{+} 511.0757$, found 511.0758 .

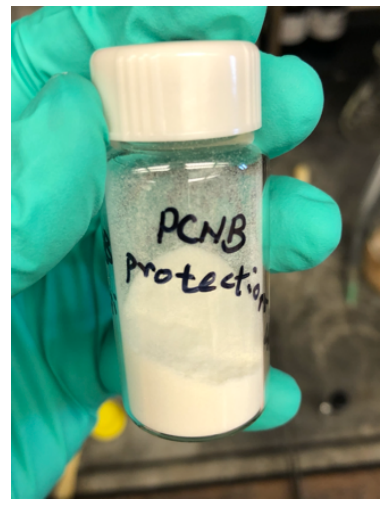

$5 \mathrm{~g}$ 


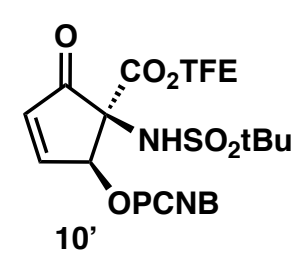

Physical State: white foam;

TLC: $R_{f}=0.45(E t O A c /$ hexanes $=1: 2)$;

${ }^{1} \mathrm{H}$ NMR $(600 \mathrm{MHz}$, Chloroform- $d$ ) $\delta 8.24(\mathrm{~d}, J=8.5 \mathrm{~Hz}, 2 \mathrm{H}), 7.76(\mathrm{~d}, J=8.5$ $\mathrm{Hz}, 2 \mathrm{H}), 7.73(\mathrm{dd}, J=6.1,2.6 \mathrm{~Hz}, 1 \mathrm{H}), 6.68(\mathrm{dd}, J=6.1,1.8 \mathrm{~Hz}, 1 \mathrm{H}), 5.97(\mathrm{dd}$, $J=2.6,1.8 \mathrm{~Hz}, 1 \mathrm{H}), 4.80-4.73(\mathrm{~m}, 1 \mathrm{H}), 4.63(\mathrm{~s}, 1 \mathrm{H}), 4.55-4.48(\mathrm{~m}, 1 \mathrm{H}), 1.36(\mathrm{~s}, 9 \mathrm{H})$;

${ }^{13} \mathrm{C}$ NMR $\left(150 \mathrm{MHz}, \mathrm{CDCl}_{3}\right) \delta 194.7,166.2,165.8,155.9,136.6,132.5,132.4(2 \mathrm{C}), 131.1$ (2C), $122.3\left(\mathrm{q}, J_{C-F}=277.4 \mathrm{~Hz}\right), 118.0,117.3,76.6,69.5,62.3\left(\mathrm{q}, J_{C-F}=37.5 \mathrm{~Hz}\right), 61.5,24.4(3 \mathrm{C})$;

19F NMR $\left(376 \mathrm{MHz}, \mathrm{CDCl}_{3}\right) \delta-73.9$;

HRMS (m/z): calculated for $\mathrm{C}_{20} \mathrm{H}_{19} \mathrm{~F}_{3} \mathrm{~N}_{2} \mathrm{O}_{7} \mathrm{~S}[\mathrm{M}+\mathrm{Na}]^{+} 511.0757$, found 511.0765 . 


\section{Compound s1 and s1'}

Experimental: $10(2.69 \mathrm{~g}, 5.51 \mathrm{mmol})$ and $\mathrm{CeCl}_{3} \cdot 7 \mathrm{H}_{2} \mathrm{O}(12.3 \mathrm{~g}, 33.1 \mathrm{mmol}, 6.0$ equiv) were dissolved in $\mathrm{MeOH}(350 \mathrm{~mL}, 0.016 \mathrm{M})$ then cooled to $-60^{\circ} \mathrm{C} . \mathrm{NaBH}_{4}(1.26 \mathrm{~g}, 33.1 \mathrm{mmol}, 6.0$ equiv) was added portionwise. After $30 \mathrm{~min}$ at $-60^{\circ} \mathrm{C}$, the reaction mixture was quenched with acetone $(20 \mathrm{~mL})$ and stirred for $20 \mathrm{~min}$. Silica gel $(30 \mathrm{~g})$ was added to the mixture, then the solvent was removed via rotary evaporation. The residual dry powder was directly dry-loaded and purified via column chromatography $\left(\mathrm{SiO}_{2}\right.$, acetone: $\left.\mathrm{CH}_{2} \mathrm{Cl}_{2}=1: 30\right)$ to give product $\mathbf{s} 1(2.02 \mathrm{~g}, 4.13 \mathrm{mmol})$ in $75 \%$ yield and s1' $(0.2 \mathrm{~g}, 0.41 \mathrm{mmol})$ in $7.5 \%$ yield.

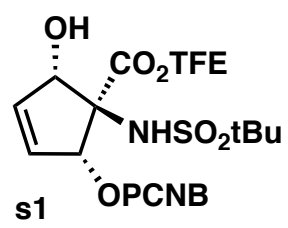

'H NMR $\left(600 \mathrm{MHz}, \mathrm{CDCl}_{3}\right) \delta 8.09(\mathrm{~d}, J=8.1 \mathrm{~Hz}, 2 \mathrm{H}), 7.75(\mathrm{~d}, J=8.2 \mathrm{~Hz}, 2 \mathrm{H}), 6.20-6.16(\mathrm{~m}$, $1 \mathrm{H}), 6.07(\mathrm{~s}, 1 \mathrm{H}), 5.99-5.95(\mathrm{~m}, 1 \mathrm{H}), 5.93(\mathrm{~d}, J=1.8 \mathrm{~Hz}, 1 \mathrm{H}), 5.29-5.26(\mathrm{~m}, 1 \mathrm{H}), 4.66-4.59$ $(\mathrm{m}, 1 \mathrm{H}), 4.38-4.30(\mathrm{~m}, 1 \mathrm{H}), 3.73(\mathrm{~d}, J=5.0 \mathrm{~Hz}, 1 \mathrm{H}), 1.51(\mathrm{~s}, 9 \mathrm{H})$;

${ }^{13} \mathrm{C}$ NMR (150 MHz, $\left.\mathrm{CDCl}_{3}\right) \delta 167.1,166.0,136.8,132.5(2 \mathrm{C}), 132.1,130.6(2 \mathrm{C}), 127.6,122.7$ (q, $\left.J_{C-F}=277.3 \mathrm{~Hz}\right), 117.8,117.6,86.8,81.6,80.8,61.41\left(\mathrm{q}, J_{C-F}=37.1 \mathrm{~Hz}\right), 60.8,24.4(3 C)$;

19F NMR (376 MHz, $\left.\mathrm{CDCl}_{3}\right) \delta-73.5$

HRMS (m/z): calculated for $\mathrm{C}_{20} \mathrm{H}_{21} \mathrm{~F}_{3} \mathrm{~N}_{2} \mathrm{O}_{7} \mathrm{~S}[\mathrm{M}+\mathrm{H}]+491.1094$, found 491.1096. 


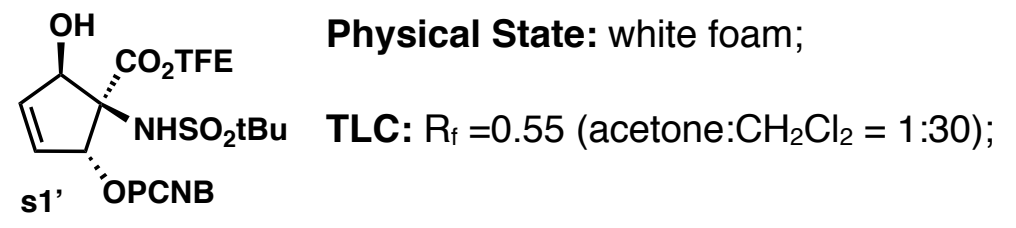

${ }^{1} \mathrm{H}$ NMR $\left(600 \mathrm{MHz}, \mathrm{CDCl}_{3}\right) \delta 8.08(\mathrm{~d}, J=8.5 \mathrm{~Hz}, 2 \mathrm{H}), 7.75(\mathrm{~d}, J=8.5 \mathrm{~Hz}, 2 \mathrm{H}), 6.39$ (ddd, $J=6.1$, 2.8, $1.8 \mathrm{~Hz}, 1 \mathrm{H}), 6.28(\mathrm{~d}, J=1.5 \mathrm{~Hz}, 1 \mathrm{H}), 6.00(\mathrm{dd}, J=6.1,1.7 \mathrm{~Hz}, 1 \mathrm{H}), 5.74(\mathrm{~s}, 1 \mathrm{H}), 5.38(\mathrm{~s}, 1 \mathrm{H})$, $4.50-4.43(\mathrm{~m}, 1 \mathrm{H}), 4.43-4.36(\mathrm{~m}, 1 \mathrm{H}), 3.46(\mathrm{~s}, 1 \mathrm{H}), 1.50(\mathrm{~s}, 9 \mathrm{H})$;

${ }^{13} \mathrm{C} \mathrm{NMR}\left(150 \mathrm{MHz}, \mathrm{CDCl}_{3}\right) \delta 167.3,165.5,137.3,132.7,132.4(2 \mathrm{C}), 130.5(3 \mathrm{C}), 122.6\left(\mathrm{q}, J_{C-F}=\right.$ $277.4 \mathrm{~Hz}), 117.9,117.3,84.4,77.6,72.9,61.5\left(\mathrm{q}, J_{C-F}=37.2 \mathrm{~Hz}\right), 60.9,24.2(3 \mathrm{C})$;

${ }^{19}$ F NMR (376 MHz, $\left.\mathrm{CDCl}_{3}\right) \delta-73.5$

HRMS (m/z): calculated for $\mathrm{C}_{20} \mathrm{H}_{21} \mathrm{~F}_{3} \mathrm{~N}_{2} \mathrm{O}_{7} \mathrm{~S}[\mathrm{M}+\mathrm{Na}]+513.0914$, found 513.0919. 


\section{Compound 11}

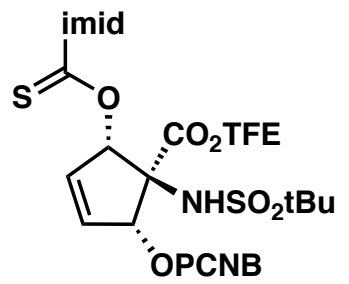

Experimental: To a solution of allylic alcohol s1 $(2.27 \mathrm{~g}, 4.63 \mathrm{mmol})$ in $\mathrm{MeCN}(46 \mathrm{~mL}, 0.1 \mathrm{M})$ was added TCDI (1.65 g, $9.26 \mathrm{mmol}, 2$ equiv) under argon. The reaction was stirred at it overnight. The reaction mixture was directly loaded onto a pad of silica gel and washed with EtOAc/hexanes $(4: 1,300 \mathrm{~mL})$ to remove polar impurities. The filtrate was concentrated via rotary evaporation and the residue was purified via column chromatography $\left(\mathrm{SiO}_{2}\right.$, EtOAc/hexanes =1:4 - 1:2) to give product 11 as colorless foam $(2.44 \mathrm{~g}, 4.08 \mathrm{mmol})$ in $88 \%$ yield.

Physical State: colorless foam;

TLC: $R_{f}=0.4($ EtOAc/hexanes = 1:1);

${ }^{1} \mathrm{H}$ NMR $\left(600 \mathrm{MHz}, \mathrm{CDCl}_{3}\right) \delta 8.18(\mathrm{~s}, 1 \mathrm{H}), 8.06(\mathrm{~d}, J=8.5 \mathrm{~Hz}, 2 \mathrm{H}), 7.76(\mathrm{~d}, J=8.5 \mathrm{~Hz}, 2 \mathrm{H}), 7.45$ $(\mathrm{s}, 1 \mathrm{H}), 7.02-6.97(\mathrm{~m}, 1 \mathrm{H}), 6.76(\mathrm{~d}, J=1.6 \mathrm{~Hz}, 1 \mathrm{H}), 6.38(\mathrm{dt}, J=6.1,1.7 \mathrm{~Hz}, 1 \mathrm{H}), 6.37(\mathrm{~s}, 1 \mathrm{H})$, $6.29(\mathrm{q}, J=1.7 \mathrm{~Hz}, 1 \mathrm{H}), 6.27(\mathrm{dt}, J=6.2,1.7 \mathrm{~Hz}, 1 \mathrm{H}), 4.73-4.66(\mathrm{~m}, 1 \mathrm{H}), 4.59-4.52(\mathrm{~m}, 1 \mathrm{H})$, $1.43(\mathrm{~s}, 9 \mathrm{H})$;

${ }^{13} \mathrm{C} \mathrm{NMR}\left(150 \mathrm{MHz}, \mathrm{CDCl}_{3}\right) \delta 182.8,166.2,164.9,136.8,132.6(3 \mathrm{C}), 132.3,131.8,131.1,130.4$ $(2 \mathrm{C}), 122.4\left(\mathrm{q}, J_{C-F}=277.3 \mathrm{~Hz}\right), 118.4,117.8,117.6,89.2,83.6,76.7,62.2\left(q, J_{C-F}=37.4 \mathrm{~Hz}\right)$, $60.9,24.2(3 \mathrm{C})$;

${ }^{19}$ F NMR (376 MHz, $\left.\mathrm{CDCl}_{3}\right) \delta$-73.3;

HRMS (m/z): calculated for $\mathrm{C}_{24} \mathrm{H}_{23} \mathrm{~F}_{3} \mathrm{~N}_{4} \mathrm{O}_{7} \mathrm{~S}_{2}[\mathrm{M}+\mathrm{H}]^{+}$601.1033, found 601.1038. 


\section{Compound 12}

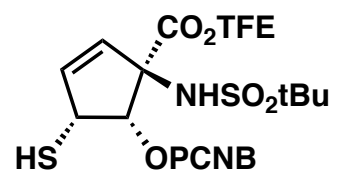

Experimental: $11(1.67 \mathrm{~g}, 2.78 \mathrm{mmol})$ was azeotroped with toluene $(50 \mathrm{~mL} \times 2)$ via rotary evaporation twice to dry the sample. BHT (31 mg, $0.14 \mathrm{mmol}, 0.05$ equiv) and toluene $(140 \mathrm{~mL}$, $0.02 \mathrm{M}$ ) were added under argon atmosphere. The mixture was heated at $115^{\circ} \mathrm{C}$ for $22 \mathrm{~h}$, then cooled to rt. The solvent was removed via rotary evaporation. The pale yellow residue was redissolved in acetone/ $\mathrm{H}_{2} \mathrm{O}(1: 1,140 \mathrm{~mL}, 0.02 \mathrm{M})$ and PTSA (1.16 g, $6.12 \mathrm{mmol}, 2.2$ equiv) was added. After $2 \mathrm{~h}$, the reaction was quenched with $\mathrm{NaHCO}_{3}$ (sat. aq., $100 \mathrm{~mL}$ ) and extracted with $\mathrm{CH}_{2} \mathrm{Cl}_{2}$ (100 mL x 3). The combined organic layers were washed with brine $(200 \mathrm{~mL})$, dried over $\mathrm{Na}_{2} \mathrm{SO}_{4}$, and concentrated via rotary evaporation. The crude product was purified via column chromatography $\left(\mathrm{SiO}_{2}\right.$, EtOAc/hexanes = 1:3) to give product $12(1.18 \mathrm{~g}, 2.34 \mathrm{mmol})$ as pale yellow foam in $84 \%$ yield.

Physical State: pale yellow foam;

TLC: $R_{f}=0.7($ EtOAc/hexanes = 1:1);

${ }^{1}$ H NMR $\left(600 \mathrm{MHz}, \mathrm{CDCl}_{3}\right) \delta 8.17(\mathrm{~d}, J=8.0 \mathrm{~Hz}, 2 \mathrm{H}), 7.77(\mathrm{~d}, J=8.1 \mathrm{~Hz}, 2 \mathrm{H}), 6.30$ (dd, $J=6.2$, $2.7 \mathrm{~Hz}, 1 \mathrm{H}), 6.13(\mathrm{~d}, J=6.2 \mathrm{~Hz}, 1 \mathrm{H}), 5.78(\mathrm{~s}, 1 \mathrm{H}), 5.59(\mathrm{~d}, J=7.3 \mathrm{~Hz}, 1 \mathrm{H}), 4.61-4.41(\mathrm{~m}, 2 \mathrm{H})$, $4.26(\mathrm{t}, J=9.3 \mathrm{~Hz}, 1 \mathrm{H}), 1.81(\mathrm{~d}, J=11.1 \mathrm{~Hz}, 1 \mathrm{H}), 1.40(\mathrm{~s}, 9 \mathrm{H})$;

${ }^{13} \mathrm{C}$ NMR (150 MHz, $\left.\mathrm{CDCl}_{3}\right) \delta 169.4,165.1,137.7,132.5(2 \mathrm{C}), 132.4,130.6(2 \mathrm{C}), 128.9,122.6$ (q, $\left.J_{C-F}=277.4 \mathrm{~Hz}\right), 117.8,117.6,80.4,74.5,62.0\left(\mathrm{q}, J_{C-F}=37.1 \mathrm{~Hz}\right), 60.2,45.4,24.3(3 \mathrm{C})$;

19F NMR (376 MHz, $\left.\mathrm{CDCl}_{3}\right) \delta-73.3 ;$

HRMS (m/z): calculated for $\mathrm{C}_{20} \mathrm{H}_{21} \mathrm{~F}_{3} \mathrm{~N}_{2} \mathrm{O}_{6} \mathrm{~S}_{2}[\mathrm{M}+\mathrm{Na}]^{+} 529.0685$, found 529.0684 . 


\section{Compound 13}<smiles>CC(=O)OC(C)=O</smiles>

Experimental: Methyl 2-acetamidoacrylate $(3.44 \mathrm{~g}, 24.06 \mathrm{mmol})$ was taken up in $\mathrm{CH}_{2} \mathrm{Cl}_{2}(120$ $\mathrm{mL}$ ) and cooled to $-78{ }^{\circ} \mathrm{C}$. Bromine (1.35 mL, 1.1 equiv) was then added dropwise and stirred at the same temperature for 15 minutes. To the yellow-orange solution was then added dropwise triethylamine (3.38 $\mathrm{mL}, 1.2$ equiv), which rendered the solution colorless. After 15 additional minutes stirring at $-78{ }^{\circ} \mathrm{C}$, the reaction was quenched with excess saturated aqueous sodium bicarbonate. Extraction was performed with $\mathrm{CH}_{2} \mathrm{Cl}_{2}(3 \times 250 \mathrm{~mL})$ and the organic portions were then washed with brine and dried over $\mathrm{Na}_{2} \mathrm{SO}_{4}$. After concentration under reduced pressure, the crude mixture was purified using silica gel column chromatography (EtOAc/Hexanes $=1: 1$ ) to yield the intermediate vinyl bromide $(5.21 \mathrm{~g}, 23.58 \mathrm{mmol})$ as a white solid in $98 \%$ yield. The spectral data matched previous reports. ${ }^{6]}$

The resulting white solid $(5.21 \mathrm{~g}, 23.58 \mathrm{mmol})$ was taken up in acetonitrile $(236 \mathrm{~mL})$ and cooled to $0{ }^{\circ} \mathrm{C}$. ditert-butyl dicarbonate $(6.5 \mathrm{~mL}, 1.2$ equiv) and DMAP (287 mg, $10 \mathrm{~mol} \%)$ were added sequentially and stirred at room temperature for 30 mins. The reaction was then diluted with EtOAc $(500 \mathrm{~mL})$ and quenched with saturated aqueous $\mathrm{NH}_{4} \mathrm{Cl}$ at $0{ }^{\circ} \mathrm{C}$. Extraction was performed with EtOAc ( $3 \times 250 \mathrm{~mL})$ and the pooled organic fractions were sequentially dried with brine and $\mathrm{Na}_{2} \mathrm{SO}_{4}$. The crude reaction mixture was concentrated under reduced pressure and purified by silica gel column chromatography (EtOAc/Hexanes $=1: 3)$ to give $13(6.43 \mathrm{~g}, 20.02 \mathrm{mmol})$ as a colorless oil in $85 \%$ yield.

Physical State: colorless oil;

TLC: $R_{f}=0.75($ EtOAc/Hexanes = 1:1);

1H NMR (600 MHz, CDCl $)$ $) 7.72(\mathrm{~s}, 1 \mathrm{H}), 3.79(\mathrm{~s}, 3 \mathrm{H}), 2.57(\mathrm{~s}, 3 \mathrm{H}), 1.45(\mathrm{~d}, J=1.3 \mathrm{~Hz}, 9 \mathrm{H})$;

${ }^{13} \mathrm{C}$ NMR $\left(150 \mathrm{MHz}, \mathrm{CDCl}_{3}\right) \delta 171.5,161.9,150.4,134.3,124.0,84.2,52.9,27.9$ (3C), 25.9;

HRMS (m/z): calculated for $\mathrm{C}_{11} \mathrm{H}_{16} \mathrm{BrNO}_{5}[\mathrm{M}+\mathrm{Na}]^{+} 344.0104$, found 344.0109 . 


\section{Compound 14}

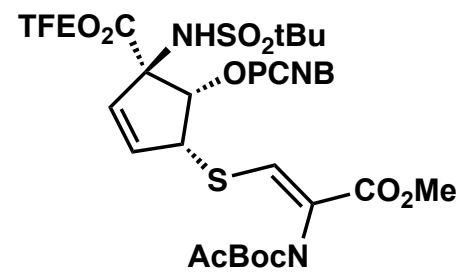

Experimental: To a mixture of vinyl bromide $13\left(898 \mathrm{mg}, 2.80 \mathrm{mmol}, 1.2\right.$ equiv) and $\mathrm{Et}_{3} \mathrm{~N}(0.64$ $\mathrm{ml}, 4.66 \mathrm{mmol}, 2.0$ equiv) in MeCN (6 mL) was added thiol 12 (1.18 g, $2.33 \mathrm{mmol})$ in MeCN (15 $\mathrm{mL}$ ), dropwise. The mixture was stirred at rt for $30 \mathrm{~min}$. Silica gel $(10 \mathrm{~g})$ was added and the solvent was removed via rotary evaporation. The residual dry powder was directly dry-loaded and purified via column chromatography $\left(\mathrm{SiO}_{2}, \mathrm{EtOAc} /\right.$ hexanes $\left.=1: 2\right)$ to give product $14(1.13 \mathrm{~g}, 1.51 \mathrm{mmol})$ in $65 \%$ yield.

Physical State: white foam;

TLC: $R_{f}=0.5($ EtOAc/hexanes = 1:1);

${ }^{1} \mathrm{H}$ NMR $\left(600 \mathrm{MHz}, \mathrm{CDCl}_{3}\right)(2: 1$ rotamers $) \delta 8.21(\mathrm{~d}, J=8.5 \mathrm{~Hz}, 2 \mathrm{H}), 8.14(\mathrm{~d}, J=8.3 \mathrm{~Hz}, 1 \mathrm{H})$, $7.78(\mathrm{~d}, J=8.4 \mathrm{~Hz}, 2 \mathrm{H}), 7.75(\mathrm{~d}, J=8.5 \mathrm{~Hz}, 1 \mathrm{H}), 7.70(\mathrm{~s}, 0.5 \mathrm{H}), 7.62(\mathrm{~s}, 1 \mathrm{H}), 6.34(\mathrm{dd}, J=6.2$, $1.4 \mathrm{~Hz}, 1 \mathrm{H}), 6.32(\mathrm{dd}, J=6.3,1.8 \mathrm{~Hz}, 0.5 \mathrm{H}), 6.24-6.21(\mathrm{~m}, 1.5 \mathrm{H}), 6.17(\mathrm{~s}, 1 \mathrm{H}), 5.88(\mathrm{~s}, 0.5 \mathrm{H})$, $5.74(\mathrm{~d}, J=7.1 \mathrm{~Hz}, 0.5 \mathrm{H}), 5.72(\mathrm{~d}, J=6.9 \mathrm{~Hz}, 1 \mathrm{H}), 4.64-4.59(\mathrm{~m}, 1.5 \mathrm{H}), 4.50-4.44(\mathrm{~m}, 1.5 \mathrm{H})$, $4.44-4.38(\mathrm{~m}, 2 \mathrm{H}), 3.70(\mathrm{~s}, 1.5 \mathrm{H}), 3.66(\mathrm{~s}, 3 \mathrm{H}), 2.53(\mathrm{~s}, 3 \mathrm{H}), 2.49(\mathrm{~s}, 1.5 \mathrm{H}), 1.47(\mathrm{~s}, 9 \mathrm{H}), 1.41(\mathrm{~s}$, $9 \mathrm{H}), 1.41(\mathrm{~s}, 4.5 \mathrm{H}), 1.40(\mathrm{~s}, 4.5 \mathrm{H})$;

${ }^{13} \mathrm{C}$ NMR $\left(150 \mathrm{MHz}, \mathrm{CDCl}_{3}\right)(2: 1$ rotamers) $\delta 171.5,171.2,168.4,168.3,165.9,165.2,162.6$, 162.4, 150.9 (2C), 142.6, 141.8, 133.6, 133.5, 133.0, 132.7 (2C), 132.6 (2C), 132.0, 131.7, 131.4 (4C), 131.0, 124.5, 124.2, $122.6\left(\mathrm{~d}, J_{C-F}=277.6 \mathrm{~Hz}\right), 122.5\left(\mathrm{~d}, J_{C-F}=277.7 \mathrm{~Hz}\right), 118.0,117.9$, 117.7, 117.6, 84.2, 84.0, 80.4 (2C), 74.6, 74.0, $62.0\left(\mathrm{~d}, J_{C-F}=37.2 \mathrm{~Hz}\right), 61.8\left(\mathrm{~d}, J_{C-F}=37.3 \mathrm{~Hz}\right)$, $60.3,60.2,54.1,53.7,52.5(2 \mathrm{C}), 28.0(3 \mathrm{C}), 27.9(3 \mathrm{C}), 26.2,26.1,24.3(6 \mathrm{C})$;

${ }^{19} \mathrm{~F}$ NMR (376 MHz, $\left.\mathrm{CDCl}_{3}\right) \delta-73.1,-73.2$;

HRMS (m/z): calculated for $\mathrm{C}_{31} \mathrm{H}_{36} \mathrm{~F}_{3} \mathrm{~N}_{3} \mathrm{O}_{11} \mathrm{~S}_{2}[\mathrm{M}+\mathrm{Na}]^{+} 770.1636$, found 770.1639 . 


\section{Compound 15}

\section{Experimental:}

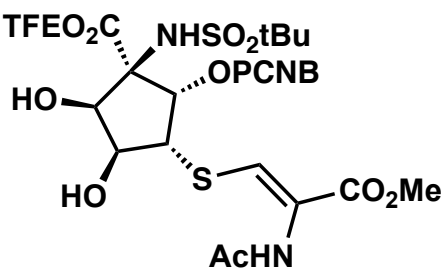

To a solution of 14 (200 mg, $0.27 \mathrm{mmol})$ in acetone/water/tBuOH $(1: 1: 1,15 \mathrm{~mL})$ was added NMO (157 mg, $1.34 \mathrm{mmol}, 5$ equiv), citric acid ( $257 \mathrm{mg}, 1.34 \mathrm{mmol}, 5$ equiv), and $\mathrm{OsO}_{4}(0.1$ equiv, added as a $2.5 \mathrm{wt} \%$ solution in $\mathrm{tBuOH})$. The reaction was heated to $35^{\circ} \mathrm{C}$ and stirred for $36 \mathrm{~h}$. Upon cooling to $\mathrm{rt}$, concentrated $\mathrm{HCl}(5 \mathrm{~mL})$ was added and the mixture was stirred for 30 minutes. $\mathrm{Na}_{2} \mathrm{~S}_{2} \mathrm{O}_{3}$ (sat. aq., $30 \mathrm{~mL}$ ) was added to quench, then $\mathrm{NaHCO}_{3}$ (sat. aq., $50 \mathrm{~mL}$ ) was added to adjust $\mathrm{pH}$ to neutral. Reaction mixture was extracted with EtOAc (100 mL x 3). Combined organic layers were washed with brine $(100 \mathrm{~mL})$, dried over $\mathrm{Na}_{2} \mathrm{SO}_{4}$, and purified by preparative HPLC. The compound was dried by lyophilizer to obtain diol 15 (148 mg, $0.22 \mathrm{mmol}$ ) in $81 \%$ yield.

Physical State: white powder;

Melting Point: $113-115^{\circ} \mathrm{C}$;

TLC: $\mathrm{R}_{\mathrm{f}}=0.5$ (acetone $\left./ \mathrm{CH}_{2} \mathrm{Cl}_{2}=1: 2\right)$;

${ }^{1} \mathrm{H}$ NMR $\left(600 \mathrm{MHz}, \mathrm{CDCl}_{3}\right) \delta 8.07(\mathrm{~d}, J=8.1 \mathrm{~Hz}, 2 \mathrm{H}), 7.73(\mathrm{~d}, J=8.4 \mathrm{~Hz}, 2 \mathrm{H}), 7.48(\mathrm{~s}, 1 \mathrm{H}), 7.04$ (s, 1H), $6.00(\mathrm{~d}, J=7.9 \mathrm{~Hz}, 1 \mathrm{H}), 5.65(\mathrm{~s}, 1 \mathrm{H}), 4.74(\mathrm{~d}, J=5.0 \mathrm{~Hz}, 1 \mathrm{H}), 4.63-4.59(\mathrm{~m}, 1 \mathrm{H}), 4.57$ - $4.49(\mathrm{~m}, 1 \mathrm{H}), 4.41-4.33(\mathrm{~m}, 1 \mathrm{H}), 3.99(\mathrm{t}, J=7.4 \mathrm{~Hz}, 1 \mathrm{H}), 3.70(\mathrm{~s}, 3 \mathrm{H}), 2.04(\mathrm{~s}, 3 \mathrm{H}), 1.44(\mathrm{~s}$, $9 \mathrm{H})$;

${ }^{13} \mathrm{C}$ NMR $\left(150 \mathrm{MHz}, \mathrm{CDCl}_{3}\right) \delta 168.5,167.7,164.0,163.4,136.8,132.5,132.4(2 \mathrm{C}), 130.6$ (2C), $122.6\left(\mathrm{~d}, J_{C-F}=277.2 \mathrm{~Hz}\right), 122.0,117.9,117.2,76.6,74.2,71.6,61.8\left(\mathrm{q}, J_{C-F}=36.8 \mathrm{~Hz}\right), 60.9$, $54.2,52.8,24.1(3 \mathrm{C}), 23.2$;

Note: One carbon was missing in ${ }^{13} \mathrm{C} N M R$. The $X$-ray crystallography assigned the structure.

${ }^{19} \mathrm{~F}$ NMR (376 MHz, $\left.\mathrm{CDCl}_{3}\right) \delta$-73.2;

HRMS (m/z): calculated for $\mathrm{C}_{26} \mathrm{H}_{30} \mathrm{~F}_{3} \mathrm{~N}_{3} \mathrm{O}_{11} \mathrm{~S}_{2}[\mathrm{M}+\mathrm{H}]^{+}$682.1347, found 682.1365. 


\section{Compound 16}

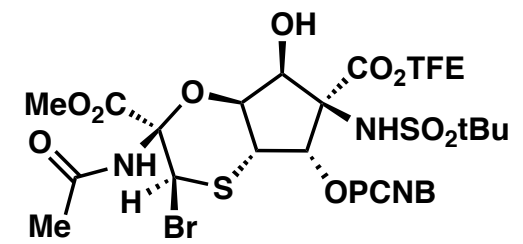

Experimental: Diol 15 (100 mg, $0.147 \mathrm{mmol})$ was taken up in $\mathrm{CHCl}_{3}(3 \mathrm{~mL}, 0.05 \mathrm{M})$ with acetic acid (10.5 $\mu \mathrm{L}, 1.25$ equiv). 5,5,-dimethyl-dibromohydantoin (53 mg, 1.25 equiv) was then added at once and stirred at rt for 5 mins. The resulting orange solution was then diluted with additional $\mathrm{CHCl}_{3}(10 \mathrm{~mL}$ total, $0.015 \mathrm{M})$ and heated at $50^{\circ} \mathrm{C}$ for 30 mins. Excess electrophilic bromide was then quenched with 1,3,5-trimethoxybenzene (74 mg, 3 equiv) and the resulting pale yellow mixture was concentrated under reduced pressure. The crude material was purified by column chromatography (1:7 EtOAc:Hexanes; then 1:10 acetone: $\mathrm{CH}_{2} \mathrm{Cl}_{2}$ ) to give product 16 (45 mg, $0.059 \mathrm{mmol}$ ) as a white solid in $40 \%$ yield.

Physical State: white solid;

Melting Point: $165-166^{\circ} \mathrm{C}$;

TLC: $\mathrm{R}_{\mathrm{f}}=0.5$ (Acetone $\left./ \mathrm{CH}_{2} \mathrm{Cl}_{2}=1: 3\right)$;

1H NMR $\left(600 \mathrm{MHz}, \mathrm{CDCl}_{3}\right) \delta 7.99(\mathrm{~d}, J=8.4 \mathrm{~Hz}, 2 \mathrm{H}), 7.76(\mathrm{~d}, J=8.4 \mathrm{~Hz}, 2 \mathrm{H}), 6.45(\mathrm{~s}, 1 \mathrm{H}), 5.85$ $(\mathrm{s}, 1 \mathrm{H}), 5.82(\mathrm{~d}, J=6.8 \mathrm{~Hz}, 1 \mathrm{H}), 5.27(\mathrm{dd}, J=12.1,4.2 \mathrm{~Hz}, 1 \mathrm{H}), 5.18(\mathrm{~s}, 1 \mathrm{H}), 5.05(\mathrm{~d}, J=4.2 \mathrm{~Hz}$, $1 \mathrm{H}), 4.42-4.35(\mathrm{~m}, 1 \mathrm{H}), 4.32-4.25(\mathrm{~m}, 1 \mathrm{H}), 4.22(\mathrm{dd}, J=12.0,6.8 \mathrm{~Hz}, 1 \mathrm{H}), 3.84(\mathrm{~s}, 3 \mathrm{H}), 3.84$ (s, 1H, overlapped), $2.04(\mathrm{~s}, 3 \mathrm{H}), 1.50(\mathrm{~s}, 9 \mathrm{H})$;

${ }^{13} \mathrm{C} \mathrm{NMR}\left(150 \mathrm{MHz}, \mathrm{CDCl}_{3}\right) \delta$ 169.2, 166.5, 165.3, 163.2, 132.6 (2C), 132.0, 130.4 (2C), 122.5 $\left(q, J_{C-F}=277.1 \mathrm{~Hz}\right), 117.7(2 \mathrm{C}), 84.7,78.6,76.9,74.0,73.3,61.9\left(\mathrm{q}, J_{C-F}=37.2 \mathrm{~Hz}\right), 61.4,56.5$, 53.8, 38.9, $24.2(3 \mathrm{C}), 23.5$;

${ }^{19}$ F NMR (376 MHz, $\left.\mathrm{CDCl}_{3}\right) \delta-73.1$;

HRMS (m/z): calculated for $\mathrm{C}_{26} \mathrm{H}_{29} \mathrm{BrF}_{3} \mathrm{~N}_{3} \mathrm{O}_{11} \mathrm{~S}_{2}[\mathrm{M}+\mathrm{H}]^{+} 760.0452$, found 760.0446 . 


\section{Compound 17}

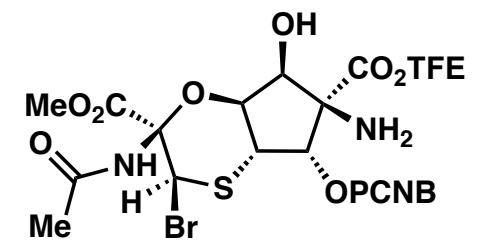

Experimental: To a solution of 16 (47 mg, $0.062 \mathrm{mmol})$ in $\mathrm{CH}_{2} \mathrm{Cl}_{2}(5 \mathrm{~mL})$ was added anisole $(0.33$ $\mathrm{mL}, 3.09 \mathrm{mmol}, 50$ equiv) under argon atmosphere. The mixture was cooled to $0{ }^{\circ} \mathrm{C}$ prior to addition of $\mathrm{TfOH}\left(54 \mu \mathrm{L}, 0.62 \mathrm{mmol}, 10\right.$ equiv). The reaction was stirred at $0{ }^{\circ} \mathrm{C}$ for $20 \mathrm{~min}$, over the course of which the reaction mixture became pink. $\mathrm{NaHCO}_{3}$ (sat. aq., $10 \mathrm{~mL}$ ) was added and the mixture was extracted with $\mathrm{CH}_{2} \mathrm{Cl}_{2}(15 \mathrm{~mL} \times 3)$. The organic layers were washed with brine $(30 \mathrm{~mL})$ and dried over $\mathrm{Na}_{2} \mathrm{SO}_{4}$. The solvent was removed via rotary evaporation. The residue was purified via column chromatography $\left(\mathrm{SiO}_{2}\right.$, acetone/ $\left.\mathrm{CH}_{2} \mathrm{Cl}_{2}=1: 6\right)$ to give product 17 (33.5 $\mathrm{mg}, 0.052 \mathrm{mmol}$ ) in $85 \%$ yield.

Physical State: white foam;

TLC: $\mathrm{R}_{\mathrm{f}}=0.4$ (acetone $\left./ \mathrm{CH}_{2} \mathrm{Cl}_{2}=1: 3\right)$;

${ }^{1}$ H NMR $\left(600 \mathrm{MHz}, \mathrm{CDCl}_{3}\right) \delta 7.99(\mathrm{~d}, J=8.4 \mathrm{~Hz}, 2 \mathrm{H}), 7.75(\mathrm{~d}, J=8.5 \mathrm{~Hz}, 2 \mathrm{H}), 6.52(\mathrm{~s}, 1 \mathrm{H}), 5.80$ $(\mathrm{s}, 1 \mathrm{H}), 5.36(\mathrm{~d}, J=5.6 \mathrm{~Hz}, 1 \mathrm{H}), 5.21$ (ddd, $J=12.1,5.0,1.3 \mathrm{~Hz}, 1 \mathrm{H}), 4.76(\mathrm{~d}, J=5.0 \mathrm{~Hz}, 1 \mathrm{H})$, $4.40-4.31(\mathrm{~m}, 2 \mathrm{H}), 4.12$ (dd, J=12.0, $5.6 \mathrm{~Hz}, 1 \mathrm{H}), 3.81(\mathrm{~s}, 3 \mathrm{H}), 2.04(\mathrm{~s}, 3 \mathrm{H})$;

${ }^{13} \mathrm{C}$ NMR $\left(150 \mathrm{MHz}, \mathrm{CDCl}_{3}\right) \delta 169.6,169.3,165.5,163.8,132.6$ (2C), 132.1, 130.3 (2C), 122.5 $\left(q, J_{C-F}=277.6 \mathrm{~Hz}\right), 117.7,117.6,84.7,80.7,79.0,72.0,68.9,61.6\left(q, J_{C-F}=37.1 \mathrm{~Hz}\right), 56.7,53.7$, 38.8, 23.5;

${ }^{19}$ F NMR (376 MHz, $\left.\mathrm{CDCl}_{3}\right) \delta-73.7$

HRMS (m/z): calculated for $\mathrm{C}_{22} \mathrm{H}_{21} \mathrm{BrF}_{3} \mathrm{~N}_{3} \mathrm{O}_{9} \mathrm{~S}[\mathrm{M}+\mathrm{H}]+640.0207$, found 640.0222. 


\section{Compound 18}

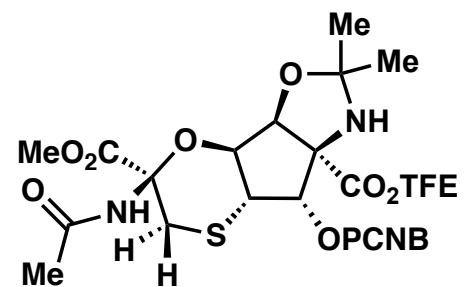

Experimental: To a solution of $17(32.7 \mathrm{mg}, 0.051 \mathrm{mmol})$ in benzene $(3 \mathrm{~mL})$ were added $\mathrm{nBu}_{3} \mathrm{SnH}(69 \mu \mathrm{L}, 0.26 \mathrm{mmol}, 5$ equiv) and AIBN (0.8 mg, $5.1 \mu \mathrm{mol}, 0.1$ equiv) under argon atmosphere. The mixture was sparged with argon for $5 \mathrm{~min}$ then heated to $85^{\circ} \mathrm{C}$. After $30 \mathrm{~min}$, the reaction was cooled to $\mathrm{rt}$ and the solvent was removed via rotary evaporation. The residue was re-dissolved in acetone $(2 \mathrm{~mL})$ and PPTS (1.3 $\mathrm{mg}, 0.1$ equiv) was added. The mixture was stirred at rt for $3 \mathrm{~h}$. $\mathrm{NaHCO}_{3}$ (sat. aq., $10 \mathrm{~mL}$ ) was added and the mixture was extracted with $\mathrm{CH}_{2} \mathrm{Cl}_{2}(15 \mathrm{~mL} \times 3)$. The organic layers were washed with brine $(20 \mathrm{~mL})$ and dried over $\mathrm{Na}_{2} \mathrm{SO}_{4}$. The solvent was removed via rotary evaporation. The residue was purified via column chromatography $\left(\mathrm{SiO}_{2}\right.$, acetone $\left./ \mathrm{CH}_{2} \mathrm{Cl}_{2}=1: 7\right)$ to give product $18(24.6 \mathrm{mg}, 0.041 \mathrm{mmol})$ in $80 \%$ yield.

Physical State: white foam;

TLC: $\mathrm{R}_{\mathrm{f}}=0.3$ (acetone $/ \mathrm{CH}_{2} \mathrm{Cl}_{2}=1: 3$ );

'H NMR (600 MHz, $\left.\left(\mathrm{CD}_{3}\right)_{2} \mathrm{CO}\right) \delta 8.07(\mathrm{~d}, J=8.5 \mathrm{~Hz}, 2 \mathrm{H}), 8.04(\mathrm{~s}, 1 \mathrm{H}), 8.00(\mathrm{~d}, J=8.5 \mathrm{~Hz}, 2 \mathrm{H})$, $5.52(\mathrm{~d}, J=4.1 \mathrm{~Hz}, 1 \mathrm{H}), 5.08-5.00(\mathrm{~m}, 2 \mathrm{H}), 4.70-4.62(\mathrm{~m}, 2 \mathrm{H}), 3.72(\mathrm{~s}, 1 \mathrm{H}), 3.66(\mathrm{~s}, 3 \mathrm{H}), 3.56$ (dd, $J=11.5,4.1 \mathrm{~Hz}, 1 \mathrm{H}), 3.10(\mathrm{~d}, J=13.6 \mathrm{~Hz}, 1 \mathrm{H}), 2.98(\mathrm{~d}, J=13.7 \mathrm{~Hz}, 1 \mathrm{H}), 1.89(\mathrm{~s}, 3 \mathrm{H}), 1.49$ $(\mathrm{s}, 3 \mathrm{H}), 1.20(\mathrm{~s}, 3 \mathrm{H})$;

${ }^{13} \mathrm{C}$ NMR $\left(150 \mathrm{MHz},\left(\mathrm{CD}_{3}\right)_{2} \mathrm{CO}\right) \delta 170.8,169.3,168.6,164.2,133.6(3 \mathrm{C}), 131.0(2 \mathrm{C}), 124.1$ (d, $\left.J_{C-F}=277.1 \mathrm{~Hz}\right), 118.5,117.8,98.0,83.4,81.0,77.9,77.2,61.5\left(\mathrm{q}, J_{C-F}=36.3 \mathrm{~Hz}\right), 52.6,41.9$, 33.4, 27.0, 26.0, 22.8;

Note: One carbon was missing in ${ }^{13} \mathrm{C} N M R$. X-ray crystallography of the next intermediate confirmed the structure.

${ }^{19}$ F NMR (376 MHz, Acetone) $\delta$-74.5;

HRMS (m/z): calculated for $\mathrm{C}_{25} \mathrm{H}_{26} \mathrm{~F}_{3} \mathrm{~N}_{3} \mathrm{O}_{9} \mathrm{~S}[\mathrm{M}+\mathrm{H}]+602.1415$, found 602.1434 . 


\section{Compound 19a and 19b}

Experimental: To a solution of $18(24 \mathrm{mg}, 0.04 \mathrm{mmol})$ in $\mathrm{MeOH}(3 \mathrm{~mL})$ was added $\mathrm{Et}_{3} \mathrm{~N}(0.28$ $\mathrm{mL}, 2.0 \mathrm{mmol}, 50$ equiv). The mixture was stirred at it overnight. Solvent was removed via rotary evaporation, then the residue was re-dissolved in $\mathrm{MeCN}(3 \mathrm{~mL})$. (+)-PSI reagent (35.7 mg, 0.08 mmol, 2 equiv) then DBN ( $14.9 \mu \mathrm{L}, 0.12 \mathrm{mmol}, 3$ equiv) were added to the reaction. The reaction was stirred at $\mathrm{rt}$ for $20 \mathrm{~min}$, then quenched with $\mathrm{NH}_{4} \mathrm{Cl}$ (sat. aq., $15 \mathrm{~mL}$ ). The mixture was extracted with $\mathrm{CH}_{2} \mathrm{Cl}_{2}(15 \mathrm{~mL} \times 4)$. The organic layers were washed with brine $(30 \mathrm{~mL})$ and dried over $\mathrm{Na}_{2} \mathrm{SO}_{4}$. The solvent was removed via rotary evaporation. The result 1:1 diastereomers were purified by SFC to give product $19 \mathrm{a}(11.7 \mathrm{mg}, 0.018 \mathrm{mmol})$ and $19 \mathrm{~b}(11.5 \mathrm{mg}, 0.018 \mathrm{mmol})$ in $90 \%$ yield.

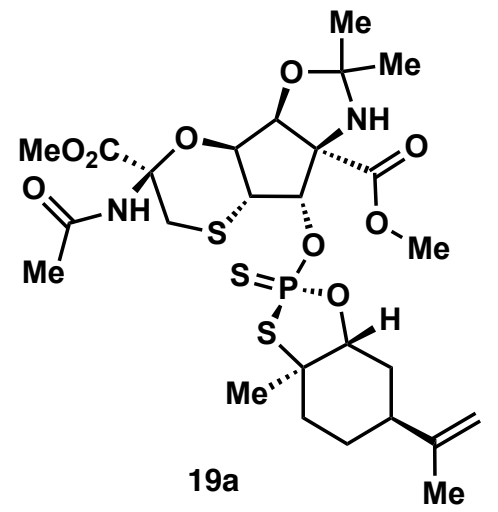

Physical State: white solid; Melting Point: $171-174^{\circ} \mathrm{C}$;

TLC: $\mathrm{R}_{\mathrm{f}}=0.35$ (Acetone $\left./ \mathrm{CH}_{2} \mathrm{Cl}_{2}=1: 3\right)$;

${ }^{1} \mathrm{H}$ NMR $\left(600 \mathrm{MHz}, \mathrm{CD}_{3} \mathrm{CN}\right) \delta 7.26(\mathrm{~s}, 1 \mathrm{H}), 5.06(\mathrm{~d}, J=1.4 \mathrm{~Hz}, 1 \mathrm{H})$, $5.00(\mathrm{dd}, J=15.8,4.5 \mathrm{~Hz}, 1 \mathrm{H}), 4.98-4.95(\mathrm{~m}, 1 \mathrm{H}), 4.82(\mathrm{~d}, J=$ $4.8 \mathrm{~Hz}, 1 \mathrm{H}), 4.75(\mathrm{dd}, J=11.8,4.8 \mathrm{~Hz}, 1 \mathrm{H}), 4.38(\mathrm{dt}, J=12.7,3.4$ $\mathrm{Hz}, 1 \mathrm{H}), 3.72(\mathrm{~s}, 3 \mathrm{H}), 3.71(\mathrm{~s}, 3 \mathrm{H}), 3.35(\mathrm{dd}, J=11.8,4.5 \mathrm{~Hz}, 1 \mathrm{H})$, $3.06(\mathrm{~d}, J=13.6 \mathrm{~Hz}, 1 \mathrm{H}), 2.97(\mathrm{~d}, J=13.8 \mathrm{~Hz}, 1 \mathrm{H}), 2.60(\mathrm{~s}, 1 \mathrm{H})$, $2.29-2.23(\mathrm{~m}, 1 \mathrm{H}), 2.06-1.99(\mathrm{~m}, 1 \mathrm{H}), 1.93-1.75(\mathrm{~m}, 4 \mathrm{H}), 1.87$ $(\mathrm{s}, 3 \mathrm{H}), 1.81(\mathrm{~s}, 3 \mathrm{H}), 1.62(\mathrm{~s}, 3 \mathrm{H}), 1.39(\mathrm{~s}, 3 \mathrm{H}), 1.13(\mathrm{~s}, 3 \mathrm{H})$;

${ }^{13} \mathrm{C}$ NMR $\left(150 \mathrm{MHz}, \mathrm{CD}_{3} \mathrm{CN}\right) \delta 172.4,170.1,168.9,146.6,112.3,97.6,87.5,83.2$ (d, JC-P $=5.0$ $\mathrm{Hz}), 83.1,81.0,78.7\left(\mathrm{~d}, J_{C-P}=6.6 \mathrm{~Hz}\right), 78.3,67.2,53.6,53.2,42.3\left(\mathrm{~d}, J_{C-P}=2.3 \mathrm{~Hz}\right), 39.8,34.2$ $\left(\mathrm{d}, J_{C-P}=9.4 \mathrm{~Hz}\right), 33.9,28.3\left(\mathrm{~d}, J_{C-P}=15.9 \mathrm{~Hz}\right), 27.4,26.0,23.8,22.9(2 \mathrm{C}), 21.9$;

${ }^{31} \mathrm{P}$ NMR $\left(162 \mathrm{MHz}, \mathrm{CD}_{3} \mathrm{CN}\right) \delta 103.0$;

HRMS (m/z): calculated for $\left.\mathrm{C}_{26} \mathrm{H}_{39} \mathrm{~N}_{2} \mathrm{O}_{9} \mathrm{PS}_{3}[\mathrm{M}+\mathrm{H}]\right]^{+} 651.1628$, found 651.1635;

$[\alpha]_{D}^{20.0}=+46.0\left(c 0.37, \mathrm{CHCl}_{3}\right)$. 


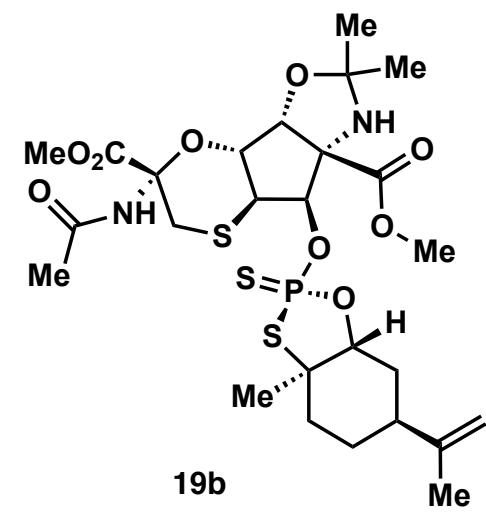

Physical State: white powder;

TLC: $\mathrm{R}_{\mathrm{f}}=0.35$ (acetone $/ \mathrm{CH}_{2} \mathrm{Cl}_{2}=1: 3$;

${ }^{1} \mathrm{H}$ NMR $\left(600 \mathrm{MHz}, \mathrm{CD}_{3} \mathrm{CN}\right) \delta 7.21(\mathrm{~s}, 1 \mathrm{H}), 5.07(\mathrm{~s}, 1 \mathrm{H}), 5.00-$ $4.94(\mathrm{~m}, 2 \mathrm{H}), 4.87(\mathrm{~d}, J=4.8 \mathrm{~Hz}, 1 \mathrm{H}), 4.56(\mathrm{dd}, J=11.7,4.8 \mathrm{~Hz}$, $1 \mathrm{H}), 4.27(\mathrm{dt}, J=12.8,3.3 \mathrm{~Hz}, 1 \mathrm{H}), 3.74(\mathrm{~s}, 3 \mathrm{H}), 3.71(\mathrm{~s}, 3 \mathrm{H}), 3.35$ (dd, $J=11.7,3.7 \mathrm{~Hz}, 1 \mathrm{H}), 3.10(\mathrm{~d}, J=13.7 \mathrm{~Hz}, 1 \mathrm{H}), 3.03(\mathrm{~s}, 1 \mathrm{H})$, $2.93(\mathrm{~d}, J=13.7 \mathrm{~Hz}, 1 \mathrm{H}), 2.61(\mathrm{~s}, 1 \mathrm{H}), 2.24(\mathrm{~d}, J=13.3 \mathrm{~Hz}, 1 \mathrm{H})$, $2.04-1.98(\mathrm{~m}, 1 \mathrm{H}), 1.93-1.75(\mathrm{~m}, 4 \mathrm{H}), 1.87(\mathrm{~s}, 3 \mathrm{H}), 1.82(\mathrm{~s}, 3 \mathrm{H})$, $1.61(\mathrm{~s}, 3 \mathrm{H}), 1.40(\mathrm{~s}, 3 \mathrm{H}), 1.13(\mathrm{~s}, 3 \mathrm{H})$;

${ }^{13} \mathrm{C}$ NMR $\left(150 \mathrm{MHz}, \mathrm{CD}_{3} \mathrm{CN}\right) \delta 172.3,170.0,169.0,146.8,112.2,97.7,87.6,83.8,82.6\left(\mathrm{~d}, J_{C-P}=\right.$ $9.2 \mathrm{~Hz}$ ), 80.4, $78.7\left(\mathrm{~d}, J_{C-P}=7.0 \mathrm{~Hz}\right), 77.4,66.0,53.3,53.2,41.8\left(\mathrm{~d}, J_{C-P}=2.0 \mathrm{~Hz}\right), 39.9,34.3(\mathrm{~d}$, $\left.J_{C-P}=9.7 \mathrm{~Hz}\right), 33.5,28.3\left(\mathrm{~d}, J_{C-P}=15.8 \mathrm{~Hz}\right), 27.0,26.0,23.8,22.9,22.8,21.8$;

${ }^{31}$ P NMR (162 MHz, $\left.\mathrm{CD}_{3} \mathrm{CN}\right) \delta$ 103.0;

HRMS (m/z): calculated for $\mathrm{C}_{26} \mathrm{H}_{39} \mathrm{~N}_{2} \mathrm{O}_{9} \mathrm{PS}_{3}[\mathrm{M}+\mathrm{H}]+651.1628$, found 651.1645;

$[\alpha]_{D}^{20.0}=+29.0\left(c 0.30, \mathrm{CHCl}_{3}\right)$. 
$(+)$ and (-)-tagetitoxin (1)<smiles>CC(=O)NC1(C(=O)O)CSC2C(O)OC(C(OP(=O)([O-])O)C2O)C1(N)C(=O)O</smiles>

$(-)-1$<smiles>CC(=O)NC1(C(=O)O)CSC2C(O)C(OP(=O)([O-])O)C(C1O)C([NH3+])(C(=O)O)C2O</smiles>

$(+)-1$

Experimental: To a solution of 19a $(11.7 \mathrm{mg}, 0.018 \mathrm{mmol})$ in $\mathrm{MeCN}(2 \mathrm{~mL})$ was added $\mathrm{SeO}_{2}$ (30.0 mg, $0.27 \mathrm{mmol}, 15$ equiv) at $0{ }^{\circ} \mathrm{C} .3 \mathrm{~min}$ later, the mixture was directly loaded into a column $\left(\mathrm{SiO}_{2}\right.$, acetone $\left./ \mathrm{CH}_{2} \mathrm{Cl}_{2}=1: 2\right)$ to remove the excess $\mathrm{SeO}_{2}$. After removed the solvent in vacuo, the residue (see picture below) was used to the next step directly.

The crude material from last step was dissolved in $\mathrm{MeCN} / \mathrm{H}_{2} \mathrm{O}(1: 1,2 \mathrm{~mL})$. TMSOK (11.5 mg, $0.09 \mathrm{mmol}, 5$ equiv) was added and the mixture was stirred at it overnight. The solvent was removed by lyophilizer. The result white solid was re-dissolved in $\mathrm{H}_{2} \mathrm{O}(1 \mathrm{~mL})$ and $\mathrm{MeONH}_{2} \cdot \mathrm{HCl}$ ( $7.5 \mathrm{mg}, 0.09 \mathrm{mmol}, 5$ equiv) was added to the solution in one potion. After $30 \mathrm{~min}$, the reaction was quenched by adding excess solid $\mathrm{NaHCO}_{3}(\sim 20 \mathrm{mg})$ to adjust to neutral $\mathrm{pH}$. The result mixture was directly purified by DEAE anion exchange chromatography (see pictures below, washed by $\mathrm{H}_{2} \mathrm{O}$ and eluted with $0.2 \mathrm{M} \mathrm{NH}_{4} \mathrm{HCO}_{3}, 0.5 \mathrm{M} \mathrm{NH}_{4} \mathrm{HCO}_{3}$ aqueous solution) to give (-)$1(5.4 \mathrm{mg}, 0.013 \mathrm{mmol})$ in $72 \%$ yield.

(+)-1 (5.2 mg, $0.012 \mathrm{mmol})$ was obtained from $19 \mathrm{~b}(11.5 \mathrm{mg}, 0.018 \mathrm{mmol})$ by the same procedure above in $70 \%$ yield.

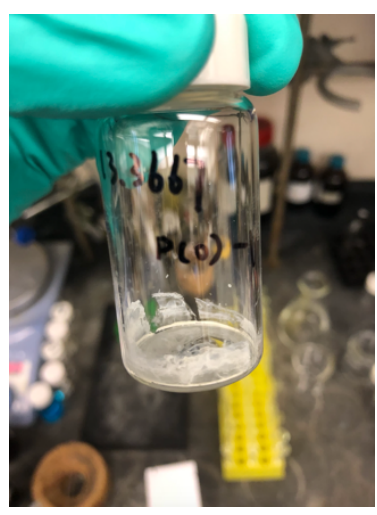

a) crude residue after $\mathrm{SeO}_{2}$ reaction

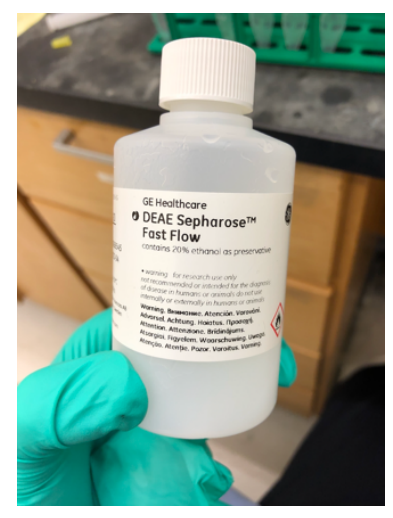

b) DEAE resin

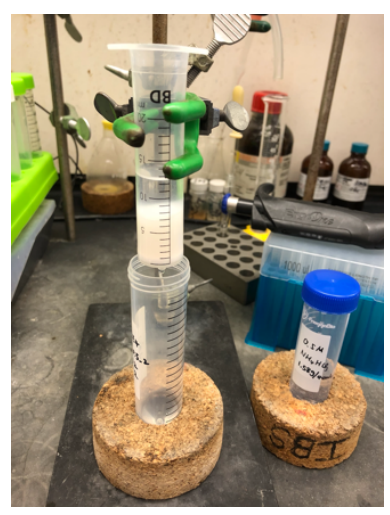

c) anion exchange chromatography purification

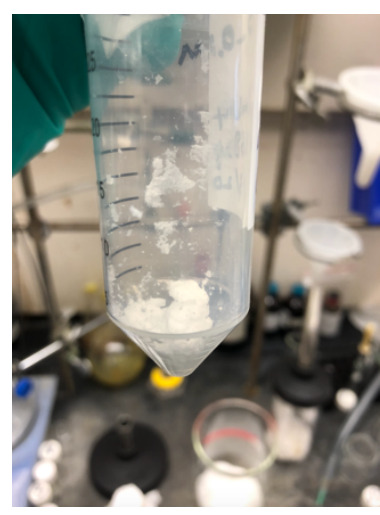

d) product after lyophilization 
Physical State: white powder;

1H NMR $\left(600 \mathrm{MHz}, \mathrm{D}_{2} \mathrm{O}\right) \delta 5.26(\mathrm{dd}, J=12.2,4.1 \mathrm{~Hz}, 1 \mathrm{H}), 4.72(\mathrm{dd}, J=11.4,7.6 \mathrm{~Hz}, 1 \mathrm{H}), 4.43$ (d, $J=4.1 \mathrm{~Hz}, 1 \mathrm{H}$ ), 3.40 (dd, $J=12.2,7.6 \mathrm{~Hz}, 1 \mathrm{H}$ ), 3.19 (d, $J=13.6 \mathrm{~Hz}, 1 \mathrm{H}), 3.00(\mathrm{~d}, J=13.6$ $\mathrm{Hz}, 1 \mathrm{H}), 2.01(\mathrm{~s}, 3 \mathrm{H})$;

${ }^{13} \mathrm{C}$ NMR $\left(150 \mathrm{MHz}, \mathrm{D}_{2} \mathrm{O}\right) \delta 174.4,173.6,171.5,85.3,79.9,76.3\left(\mathrm{~d}, J_{C-P}=4.6 \mathrm{~Hz}\right), 73.0,71.2$ $\left(\mathrm{d}, J_{C-P}=4.7 \mathrm{~Hz}\right), 43.6\left(\mathrm{~d}, J_{C-P}=2.6 \mathrm{~Hz}\right), 33.6,22.8$;

31P NMR (162 MHz, $\left.\mathrm{D}_{2} \mathrm{O}\right) \delta 4.16$

HRMS (m/z): calculated for $\mathrm{C}_{11} \mathrm{H}_{17} \mathrm{~N}_{2} \mathrm{O}_{11} \mathrm{PS}[\mathrm{M}+\mathrm{H}]+417.0363$, found 417.0372;

$(+)-1:[\alpha]_{D}^{20.0}=+55.6\left(c 0.036,0.05 \mathrm{M} \mathrm{NH}_{4} \mathrm{HCO}_{3}\right.$ in $\left.\mathrm{H}_{2} \mathrm{O}\right)$;

$(-)-1:[\alpha]_{D}^{20.0}=-45.8\left(c 0.048,0.05 \mathrm{M} \mathrm{NH}_{4} \mathrm{HCO}_{3}\right.$ in $\left.\mathrm{H}_{2} \mathrm{O}\right)$.

Note: As 1 is very sensitive to acid, $0.05 \mathrm{M} \mathrm{NH}_{4} \mathrm{HCO}_{3}$ was added as a $\mathrm{pH}$ buffer. 


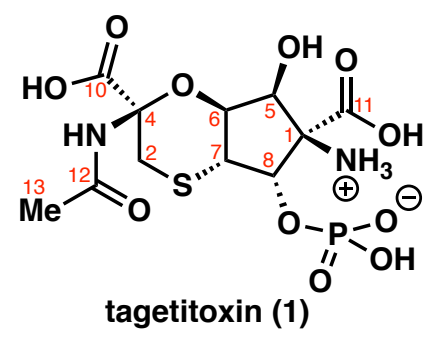

Tabulated data for Natural (Aliev ${ }^{[3]}$ ) and Synthetic Tagetitoxin ${ }^{1} \mathrm{H}$ NMR $(600 \mathrm{MHz})$

\begin{tabular}{|c|c|c|c|}
\hline & $\begin{array}{c}\text { Natural } \\
\text { Tagetitoxin } \\
\left(\mathrm{D}_{2} \mathrm{O}\right)\end{array}$ & $\begin{array}{c}\text { Synthetic } \\
\text { Tagetitoxin } \\
\left(0.05 \mathrm{M} \mathrm{NH}_{4} \mathrm{HCO}_{3}\right. \\
\left.\text { in } \mathrm{D}_{2} \mathrm{O}\right)\end{array}$ & Delta ppm \\
\hline $\mathrm{H} 2$ & $\begin{array}{c}3.25, \mathrm{~d} \\
(J=13.7 \mathrm{~Hz})\end{array}$ & $\begin{array}{c}3.19, \mathrm{~d} \\
(J=13.6 \mathrm{~Hz})\end{array}$ & 0.06 \\
\hline $\mathrm{H} 2^{\prime}$ & $\begin{array}{c}2.98, \mathrm{~d} \\
(J=13.7 \mathrm{~Hz})\end{array}$ & $\begin{array}{c}3.00, \mathrm{~d} \\
(J=13.6 \mathrm{~Hz})\end{array}$ & 0.02 \\
\hline $\mathrm{H} 5$ & $\begin{array}{c}4.46, \mathrm{~d} \\
(J=4.1 \mathrm{~Hz})\end{array}$ & $\begin{array}{c}4.43, \mathrm{~d} \\
(J=4.1 \mathrm{~Hz})\end{array}$ & 0.03 \\
\hline $\mathrm{H} 6$ & $\begin{array}{c}5.13, \mathrm{dd} \\
(J=2,4.1 \mathrm{~Hz})\end{array}$ & $\begin{array}{c}5.26, \mathrm{dd} \\
(J=12.2,4.1 \mathrm{~Hz})\end{array}$ & 0.13 \\
\hline $\mathrm{H} 7$ & $\begin{array}{c}3.48, \mathrm{dd} \\
(J=12.2,7.8 \mathrm{~Hz})\end{array}$ & $\begin{array}{c}3.40, \mathrm{dd} \\
(J=12.2,7.6 \mathrm{~Hz})\end{array}$ & 0.08 \\
\hline $\mathrm{H} 8$ & $4.73, \mathrm{dd}$ & $\begin{array}{c}4.72, \mathrm{dd} \\
(J=11.4,7.6 \mathrm{~Hz})\end{array}$ & 0.01 \\
\hline $13-\mathrm{Me}$ & $2.01, \mathrm{~s}$ & $2.01, \mathrm{~s}$ & - \\
\hline
\end{tabular}




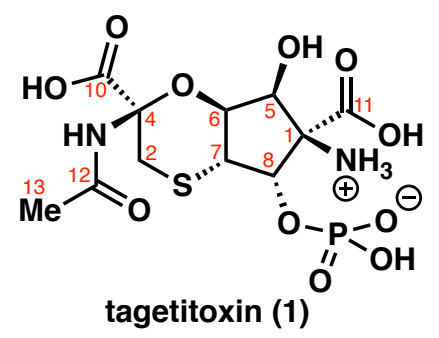

Tabulated data for Natural (Alievi3]) and Synthetic Tagetitoxin ${ }^{13} \mathrm{C}$ NMR $(150 \mathrm{MHz})$

\begin{tabular}{|c|c|c|c|}
\hline & $\begin{array}{c}\text { Natural } \\
\text { Tagetitoxin } \\
\left(\mathrm{D}_{2} \mathrm{O}\right)\end{array}$ & $\begin{array}{c}\text { Synthetic } \\
\text { Tagetitoxin } \\
\left(0.05 \mathrm{M} \mathrm{NH}_{4} \mathrm{HCO}_{3}\right. \\
\left.\text { in } \mathrm{D}_{2} \mathrm{O}\right)\end{array}$ & Delta ppm \\
\hline $\mathrm{C} 1$ & $\begin{array}{c}71.2, \mathrm{~d} \\
\left(J_{C-P}=4.9 \mathrm{~Hz}\right)\end{array}$ & $\begin{array}{c}71.2, \mathrm{~d} \\
\left(J_{C-P}=4.7 \mathrm{~Hz}\right)\end{array}$ & - \\
\hline $\mathrm{C} 2$ & 33.1 & 33.6 & 0.5 \\
\hline $\mathrm{C} 4$ & 85.6 & 85.3 & 0.3 \\
\hline $\mathrm{C} 5$ & 72.9 & 73.0 & 0.1 \\
\hline $\mathrm{C} 6$ & 79.8 & 79.9 & 0.1 \\
\hline $\mathrm{C} 7$ & $43.2, \mathrm{~d}$ & $43.6, \mathrm{~d}$ & 0.4 \\
\hline $\mathrm{C} 8$ & $\left(J_{C-P}=2.8 \mathrm{~Hz}\right)$ & $\left(J_{C-P}=2.6 \mathrm{~Hz}\right)$ & 0.7 \\
\hline $\mathrm{C} 10$ & $\left(J_{C-P}=4.0, \mathrm{~d}\right.$ & $76.3, \mathrm{~d}$ & \\
\hline $\mathrm{C} 11$ & 174.4 & $\left(J_{C-P}=4.6 \mathrm{~Hz}\right)$ & - \\
\hline $\mathrm{C} 12$ & 171.2 & 174.4 & 0.3 \\
\hline $\mathrm{C} 13$ & 173.8 & 171.5 & 0.2 \\
\hline & 22.9 & 22.8 & 0.1 \\
\hline
\end{tabular}




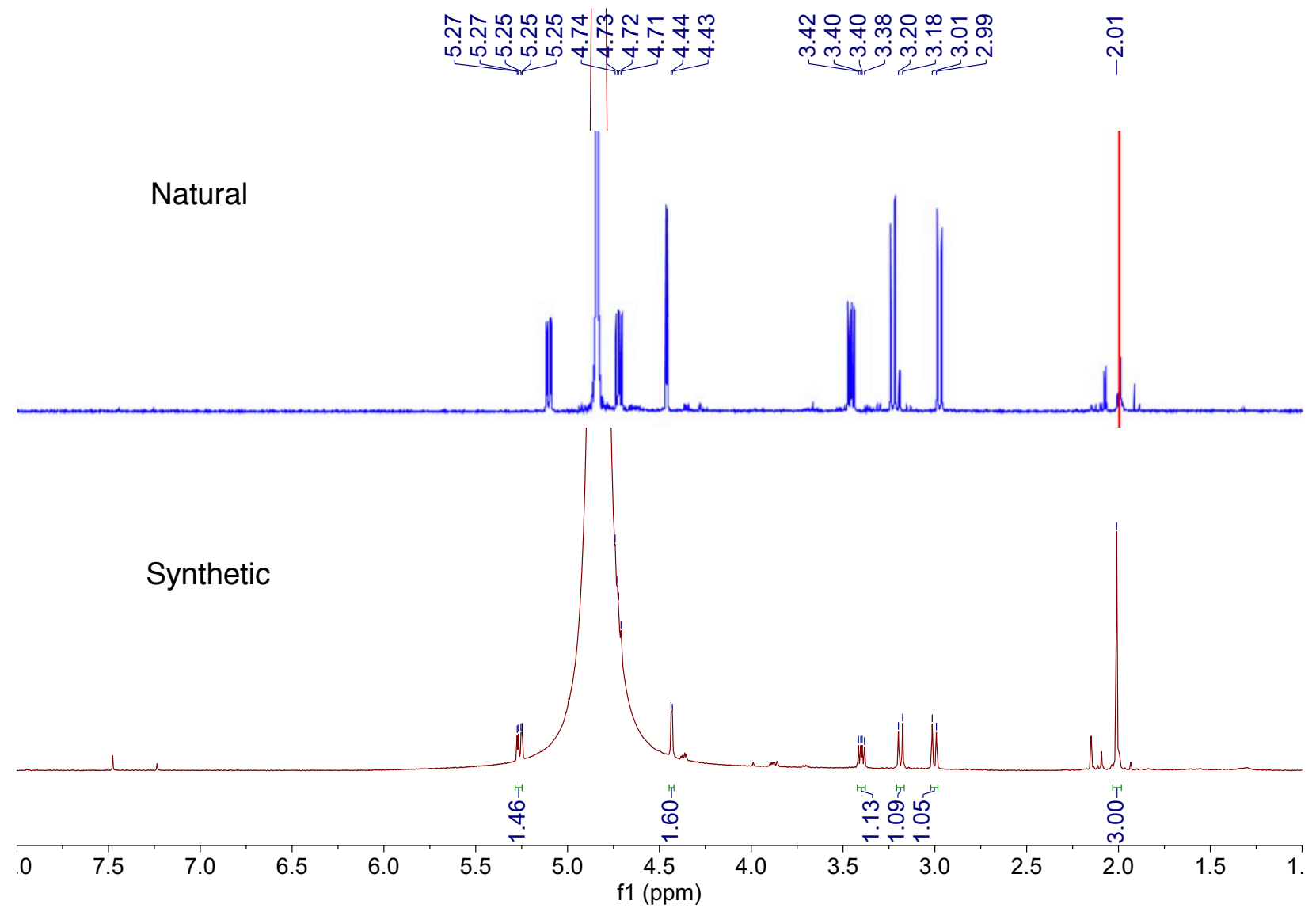

Figure S2. Comparison of ${ }^{1} \mathrm{H}$ NMR spectra of Natural (Aliev ${ }^{[3]}$ ) and Synthetic Tagetitoxin 


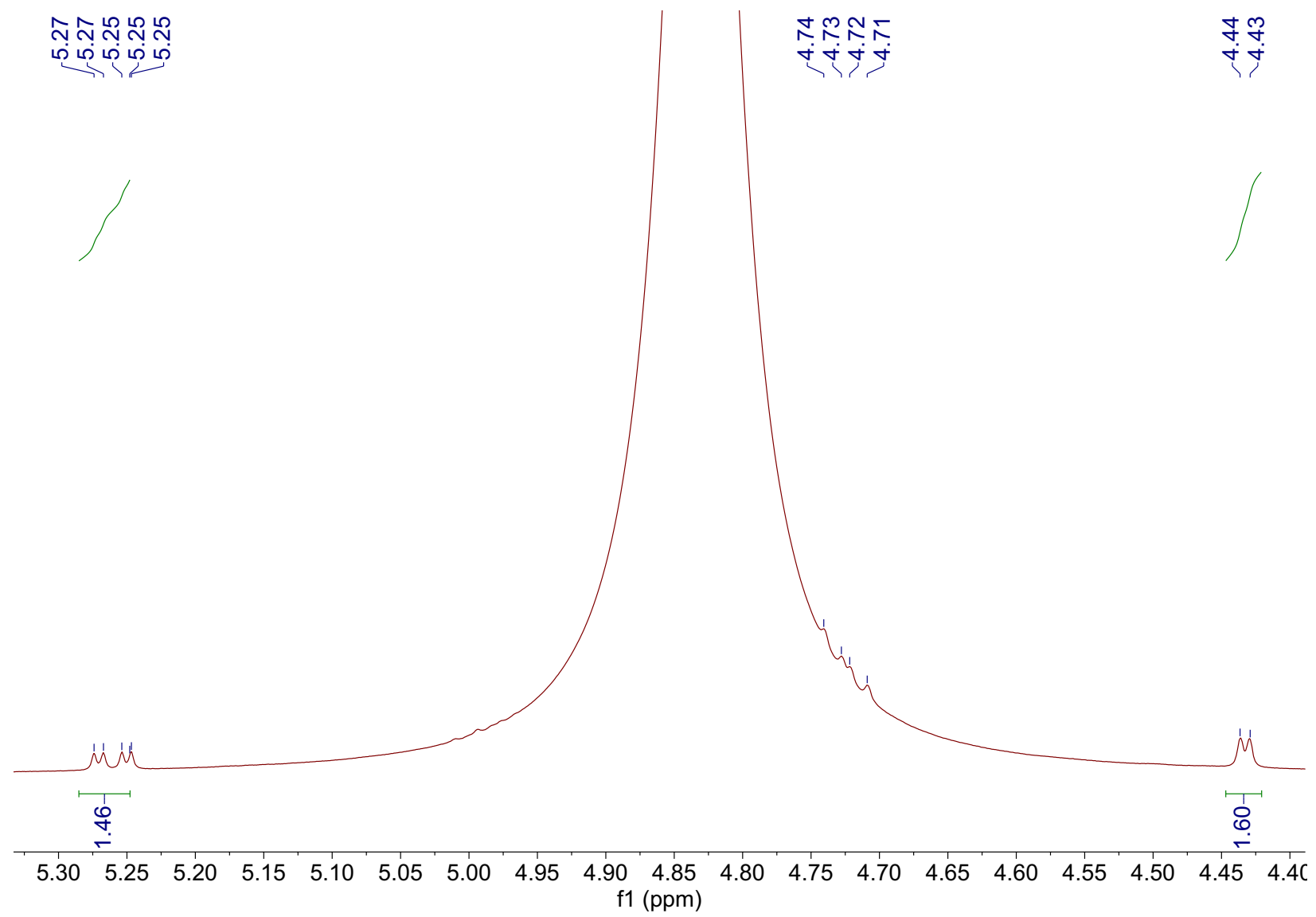

Figure S3. Zoomed in ${ }^{1} \mathrm{H}$ NMR Spectrum of Synthetic Tagetitoxin, Highlighting Resonances Covered by Solvent Peak 


\section{VI) Direct RNA Polymerase Inhibition Assay}

The RNA polymerase (RNAP) assay was obtained from Profoldin (Hudson, MA, USA) and included 100x E.coli RNAP, 100x DNA template, 100x NTPs, 10x reaction buffer, and 10x fluorescence dye. The assay protocol was modified from Kurosu et al[7]. The 100x RNAP, DNA template and NTP stock solutions were diluted in water to $10 \mathrm{x}$. The fluorescent dye was diluted with water to $1 \mathrm{x}$. RNAP Inhibitors, (+)-1, (-)-1 and rifampicin were dissolved to $10 \mathrm{mM}$ in tris $(100 \mathrm{mM}, \mathrm{pH} 8)$, these stocks were then serial diluted to concentrations between $1 \mathrm{mM}$ and $0.1 \mu \mathrm{M}$ which were then used as 10x stocks. Water (30 $\mu \mathrm{L})$, reaction buffer (10x, $6 \mu \mathrm{L})$, DNA template $(10 \mathrm{x}, 6 \mu \mathrm{L})$, NTPs $(10 \mathrm{x}, 6 \mu \mathrm{L})$ was added to a black 96 well plate (Corning). Inhibitor was added $(10 \mathrm{x}, \mu \mathrm{L})$ so that the final assay concentrations were between $.1 \mathrm{mM}$ and $0.01 \mu \mathrm{M}$. Finally, RNAP was added $(10 \mathrm{x}, 6 \mu \mathrm{L})$ and the plate was incubated at $37^{\circ} \mathrm{C}$ for 60 minutes. Fluorescent dye $(1 \mathrm{x}, 60 \mu \mathrm{L})$ was added to each well, and the plate was incubated for 15 minutes at room temperature. Relative fluorescence intensity was measured at an excitation wavelength of $485 \mathrm{~nm}$ and an emission wavelength of $528 \mathrm{~nm}$ using an EnVision Multilabel plate reader. Data processing and curve fitting was performed in Graphpad Prism. 
(+)-1

\begin{tabular}{|c|c|c|c|}
\hline Concentration $(\log (\mathrm{M}))$ & \multicolumn{3}{|c|}{ RFU } \\
\hline-4 & 157728 & 153654 & 154620 \\
\hline-5 & 158023 & 171578 & 168043 \\
\hline-6 & 171621 & 194280 & 208511 \\
\hline-7 & 205070 & 238900 & 215676 \\
\hline-8 & 211148 & 217668 & 219255 \\
\hline
\end{tabular}

\begin{tabular}{|l|r|}
\hline Best-fit values & \\
\hline Bottom & 71.02 \\
\hline Top & 99.85 \\
\hline LogIC50 & -5.854 \\
\hline HillSlope & -1.034 \\
\hline IC50 & $1.4 \mathrm{E}-06$ \\
\hline Span & 28.83 \\
\hline R squared & 0.8649 \\
\hline
\end{tabular}

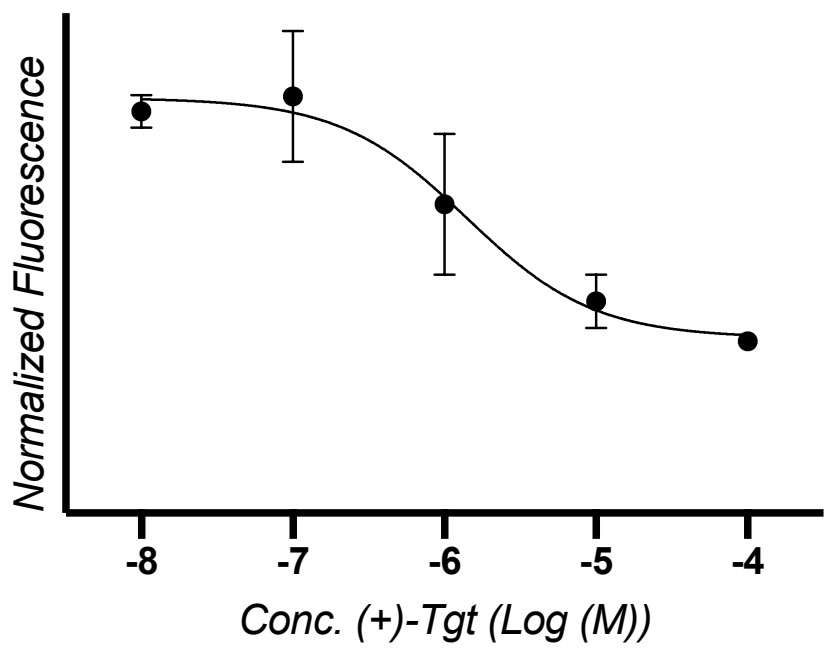


$(-)-1$

\begin{tabular}{|c|c|c|c|}
\hline Concentration $(\log (\mathrm{M}))$ & \multicolumn{3}{|c|}{ RFU } \\
\hline-4 & 259541 & 283960 & 260529 \\
\hline-5 & 245174 & 278500 & 264570 \\
\hline-6 & 256766 & 248615 & 261754 \\
\hline-7 & 230766 & 244424 & 242342 \\
\hline-8 & 242065 & 274627 & 234584 \\
\hline
\end{tabular}

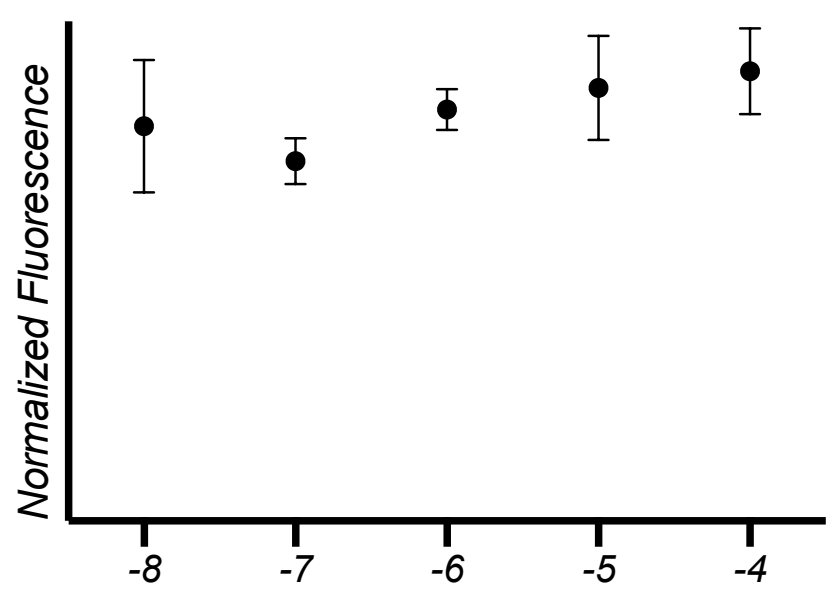

Conc. (-)-Tgt (Log (M)) 
Rifampicin (Negative control)

\begin{tabular}{|c|r|r|r|}
\hline Concentration $(\log (\mathrm{M}))$ & \multicolumn{3}{|c|}{ RFU } \\
\hline-4 & 82675 & 78553 & 76962 \\
\hline-5 & 95984 & 100393 & 90257 \\
\hline-6 & 118448 & 113599 & 113312 \\
\hline-7 & 223906 & 224286 & 174501 \\
\hline-8 & 286976 & 352570 & 246402 \\
\hline-9 & 289631 & 308029 & 290962 \\
\hline
\end{tabular}

\begin{tabular}{|l|r|}
\hline Best-fit values & \\
\hline Bottom & 86432 \\
\hline Top & 303306 \\
\hline LoglC50 & -6.871 \\
\hline HillSlope & -0.9903 \\
\hline IC50 & $1.35 \mathrm{E}-07$ \\
\hline Span & 216874 \\
\hline R squared & 0.9467 \\
\hline
\end{tabular}

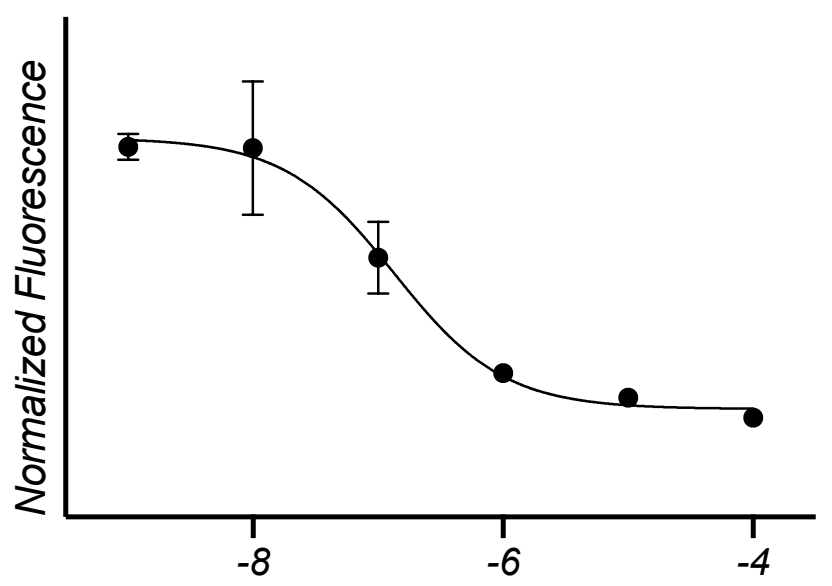

Conc. Rifampicin (Log (M)) 
VII) ${ }^{1} \mathrm{H}$ - and ${ }^{13} \mathrm{C}$-NMR Spectra 
${ }^{1} \mathrm{H}$ NMR of $7\left(600 \mathrm{MHz}, \mathrm{CDCl}_{3}\right)$

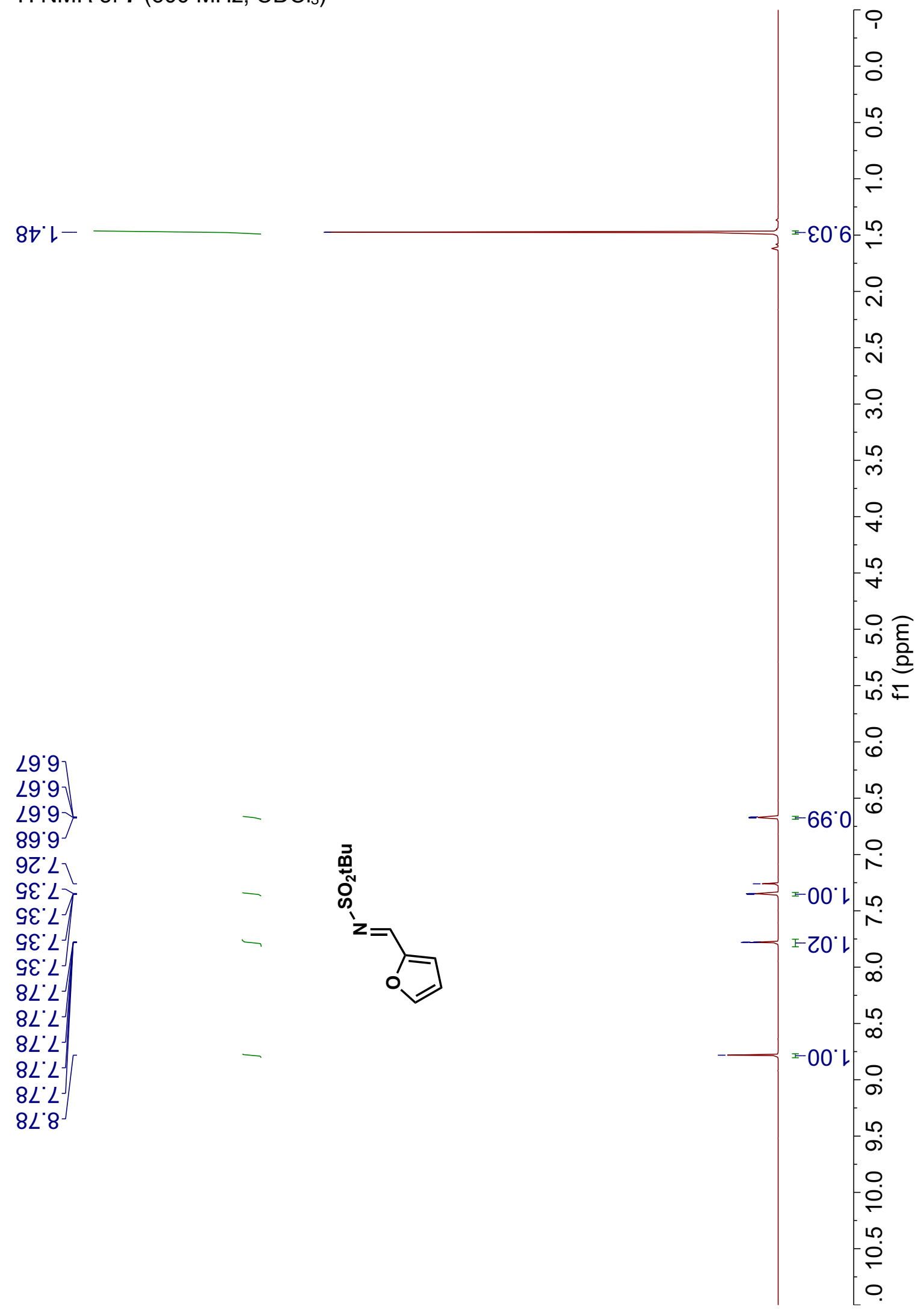


${ }^{13} \mathrm{C}$ NMR of $7\left(150 \mathrm{MHz}, \mathrm{CDCl}_{3}\right)$

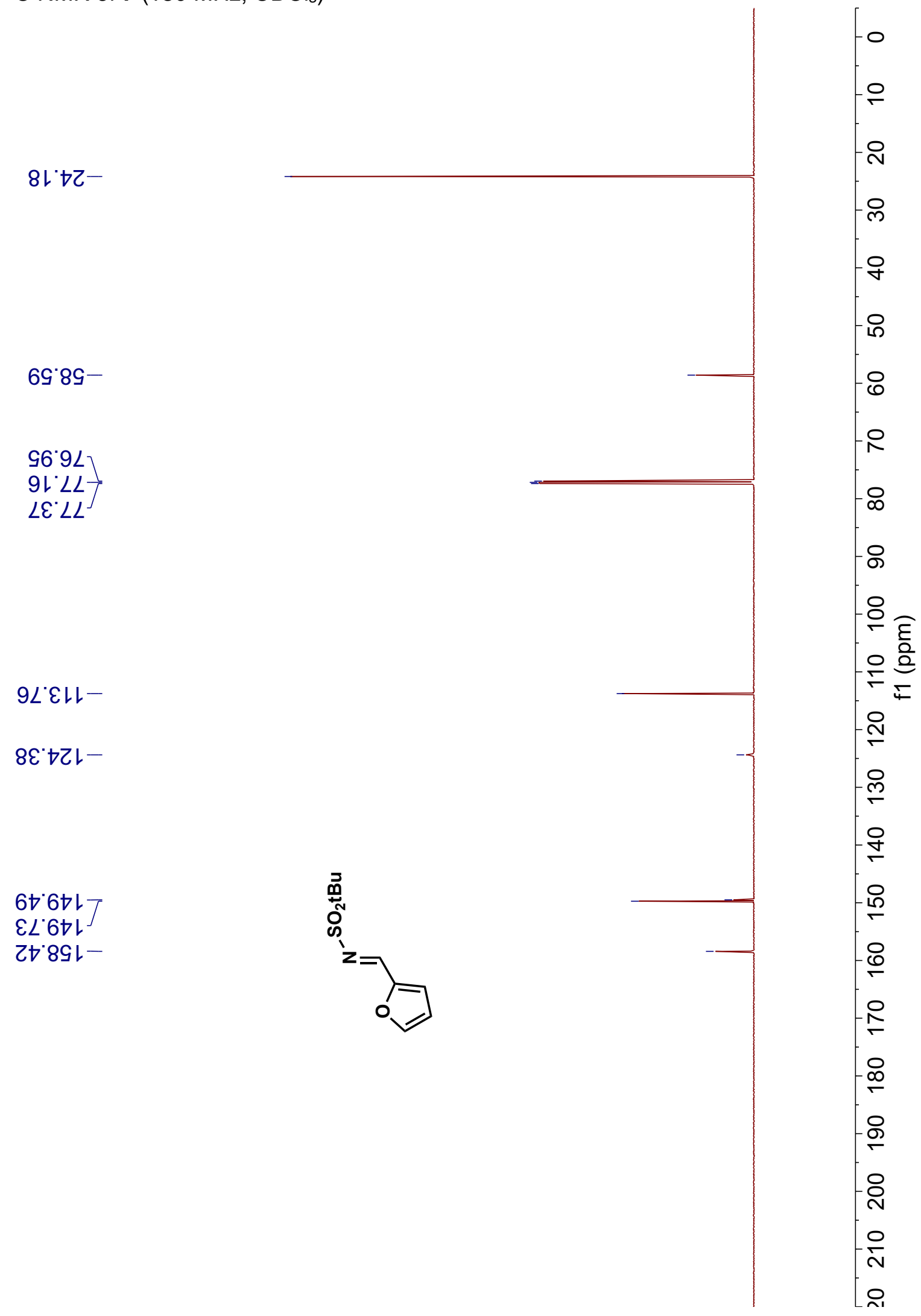


${ }^{1} \mathrm{H}$ NMR of $8\left(600 \mathrm{MHz}, \mathrm{CDCl}_{3}\right)$

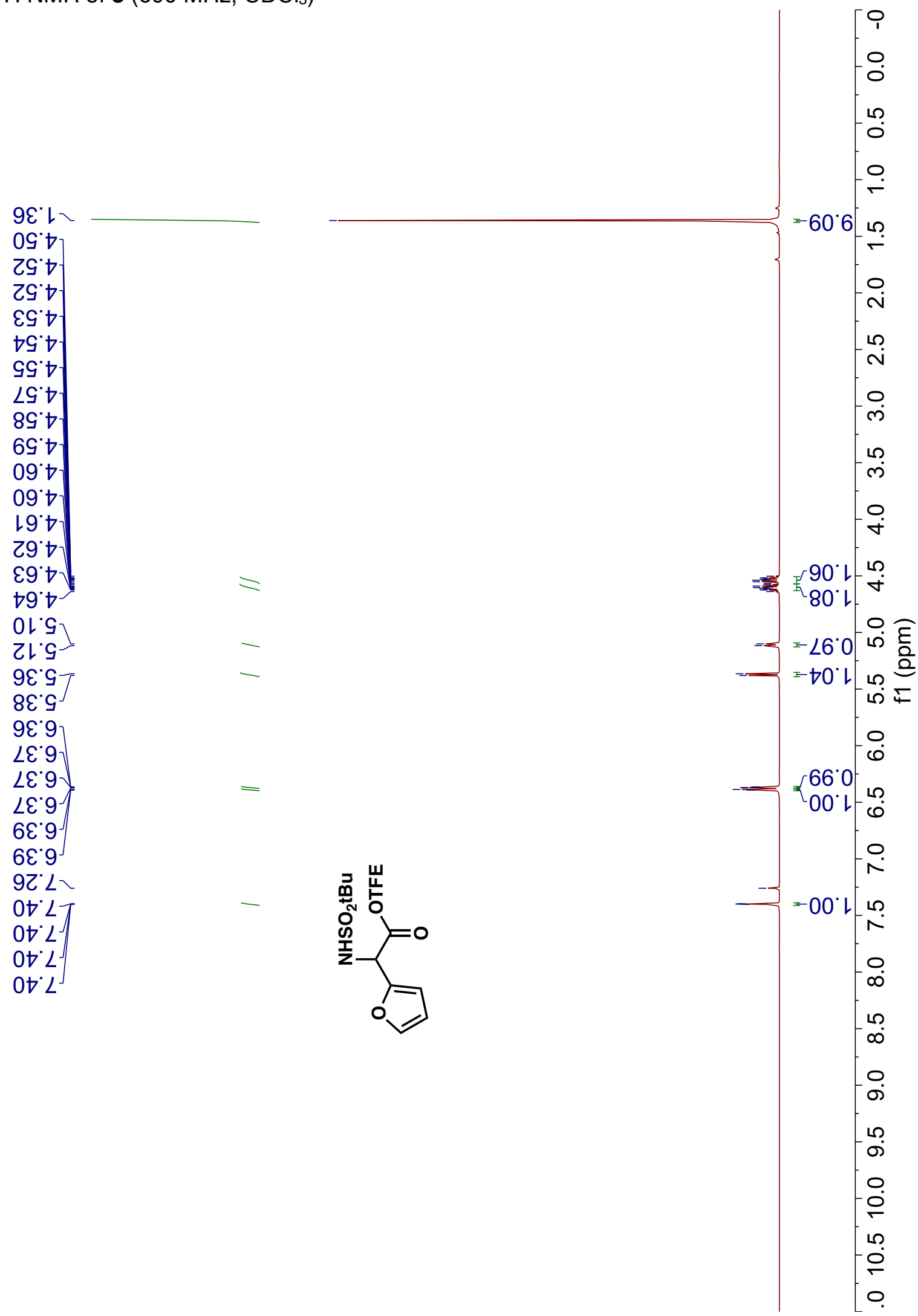


${ }^{13} \mathrm{C}$ NMR of $8\left(150 \mathrm{MHz}, \mathrm{CDCl}_{3}\right)$

乙๐॰て一

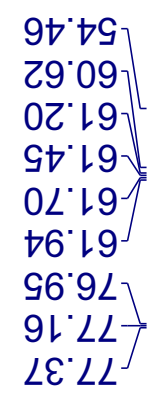

$87^{\circ} 60 \mathrm{~L}$

レ゚ルレー

8L'6L

て9レてレ

งtยてレ-

6乙'G乙レ

ง9ยヤレ-

99'レヤレ-

$80.891-$

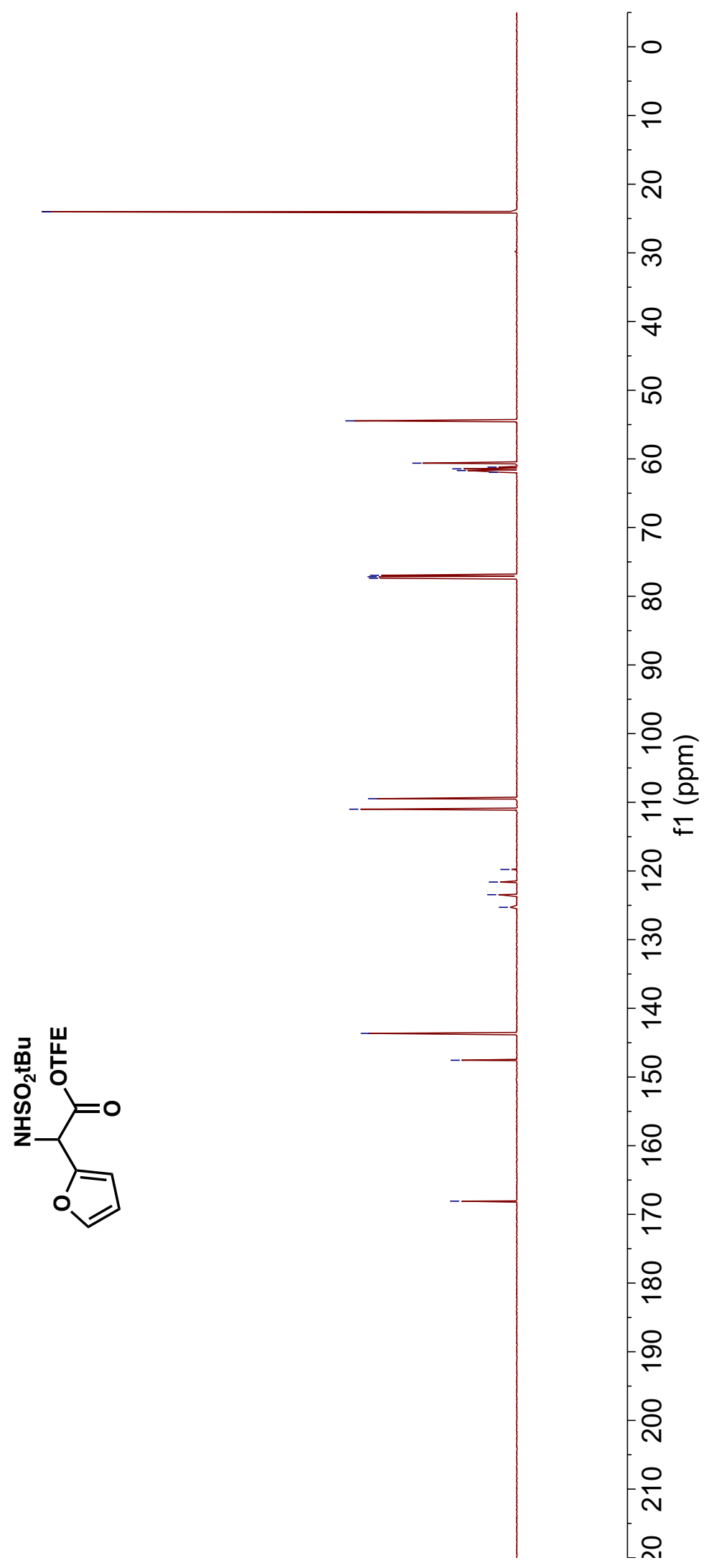


${ }^{19} \mathrm{~F}$ NMR of $8\left(376 \mathrm{MHz}, \mathrm{CDCl}_{3}\right)$

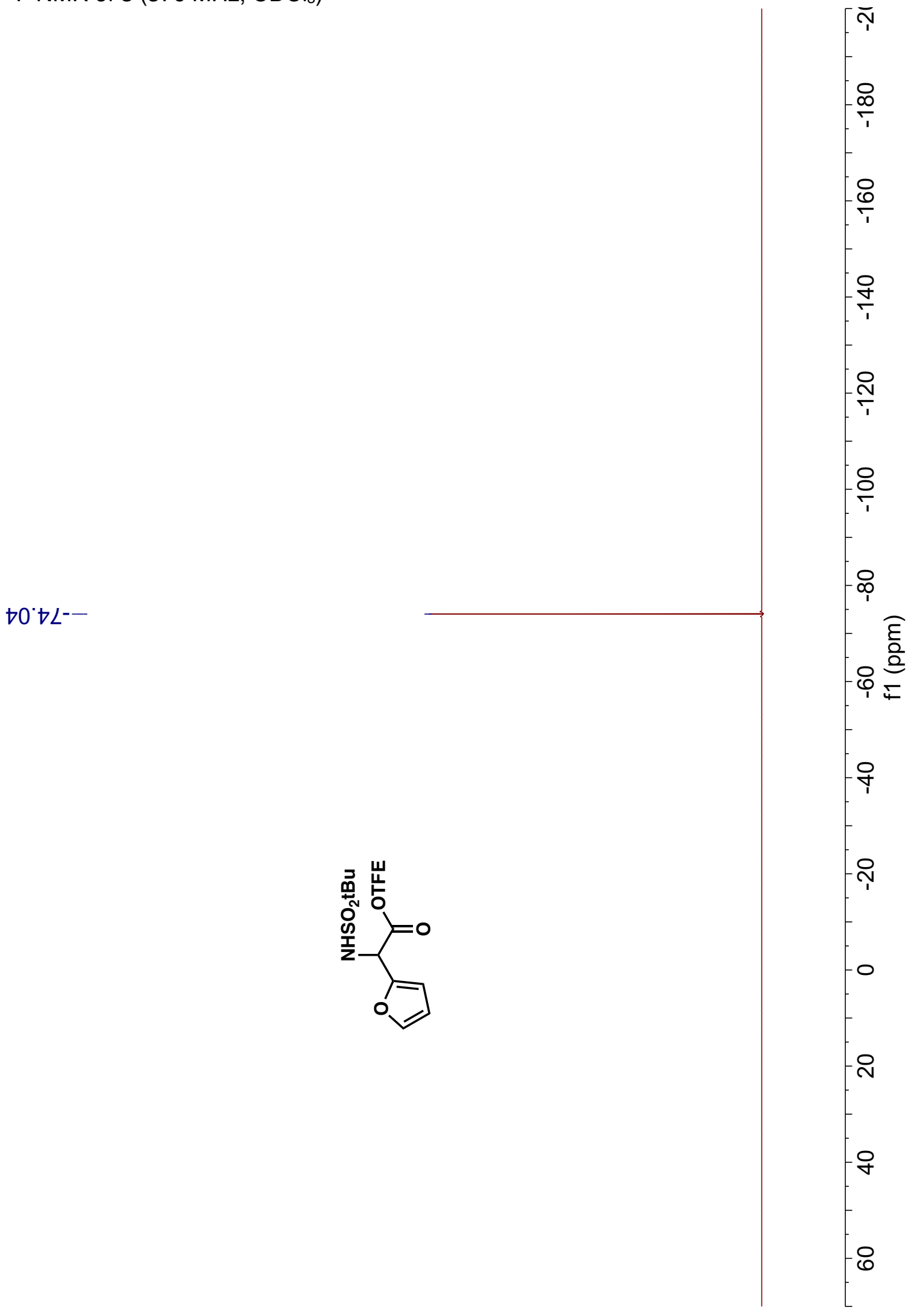




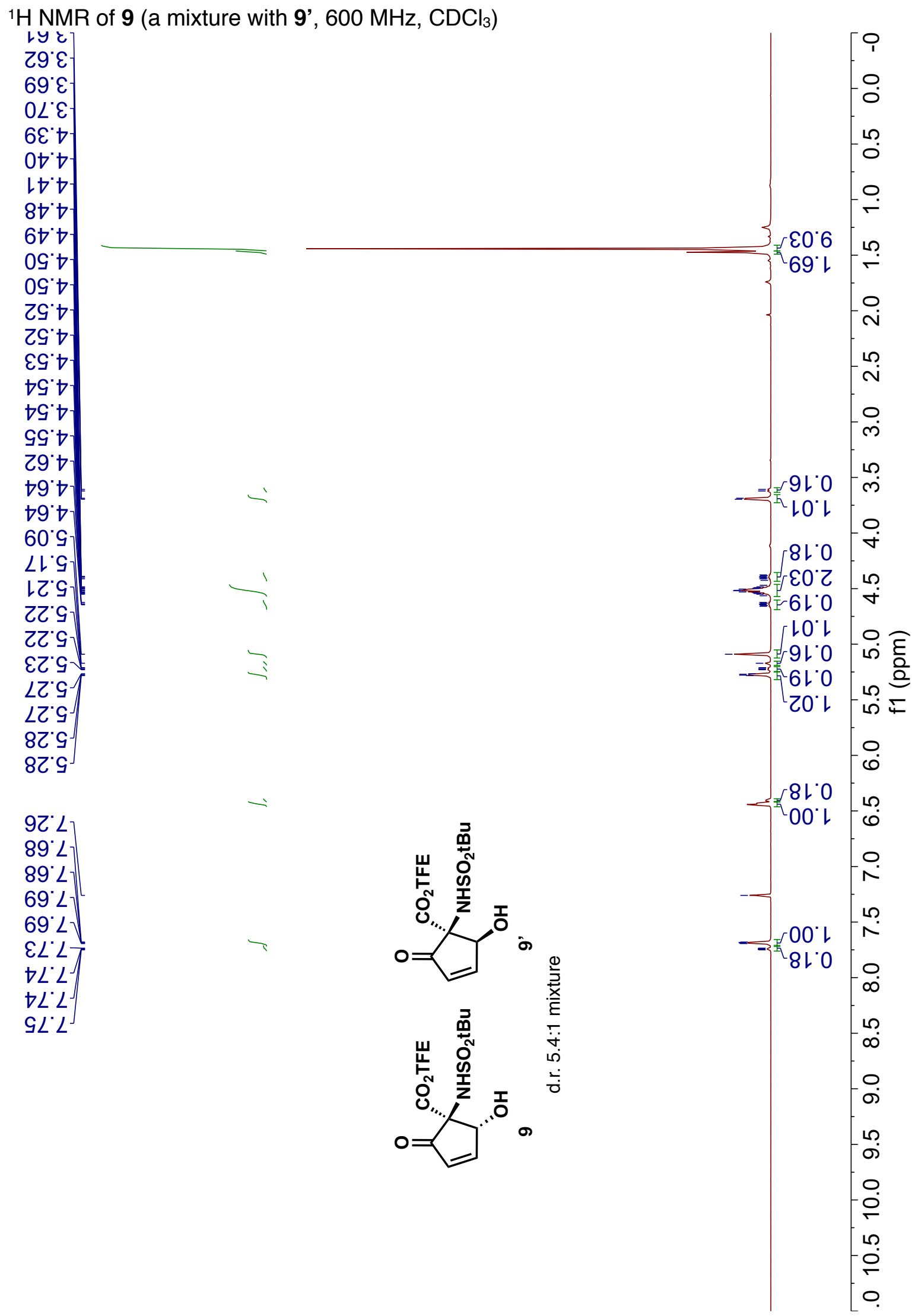


${ }^{13} \mathrm{C}$ NMR of 9 (a mixture with 9', $150 \mathrm{MHz}, \mathrm{CDCl}_{3}$ )

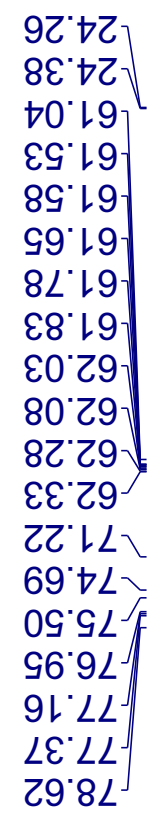

$\angle 96 \mathrm{LL}$

IG'LZL

Gะ'ยZレ -

$6 L^{\circ} \mathrm{GZL}$

89'ZEトT

$0 l \cdot \varepsilon \varepsilon l$

$\varepsilon+19 L$

9Z'Z9L

S9.991 T

$\angle Z \cdot 99 \mathrm{l}$

$\varepsilon 8 \cdot 96 \downarrow$

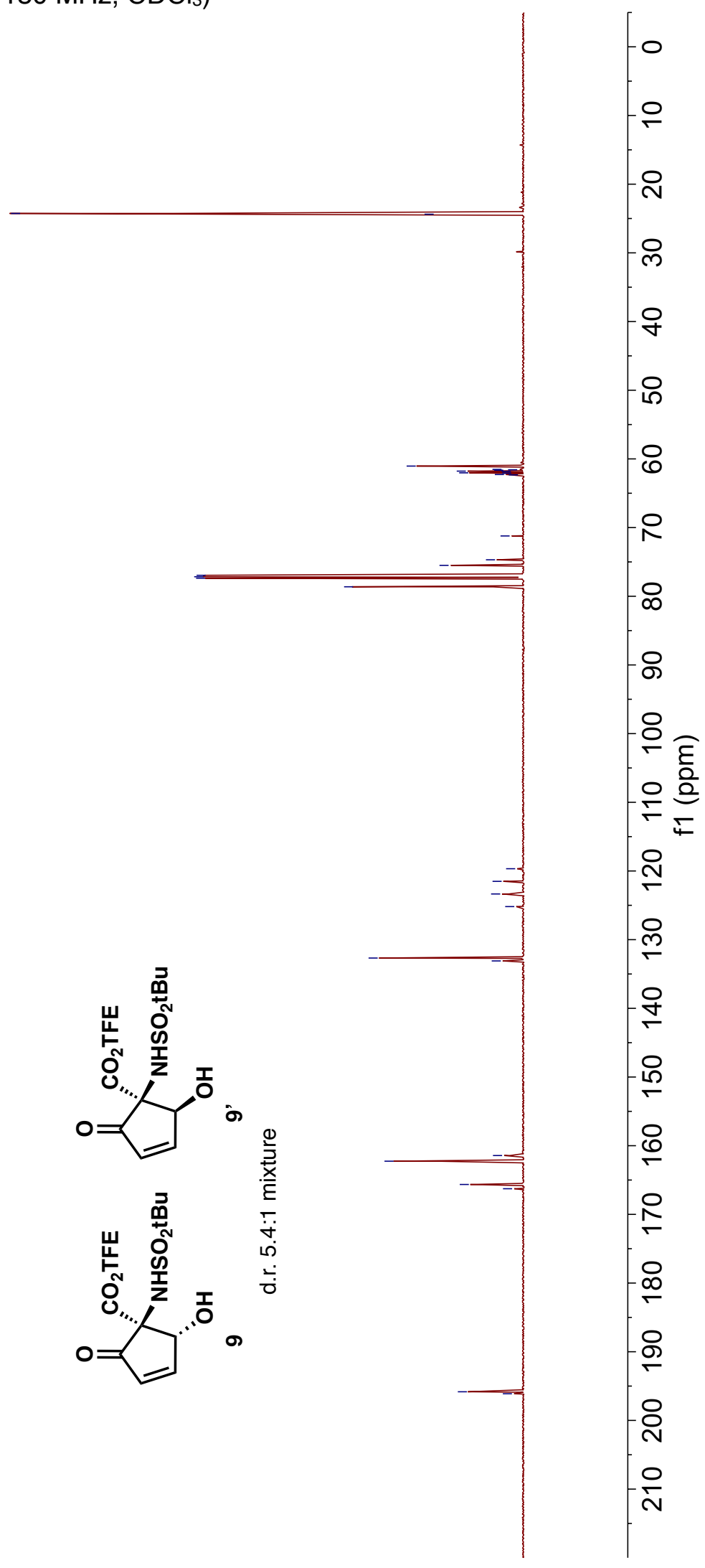


${ }^{19} \mathrm{~F}$ NMR of 9 (a mixture with $\mathbf{9}^{\prime}, 376 \mathrm{MHz}, \mathrm{CDCl}_{3}$ )

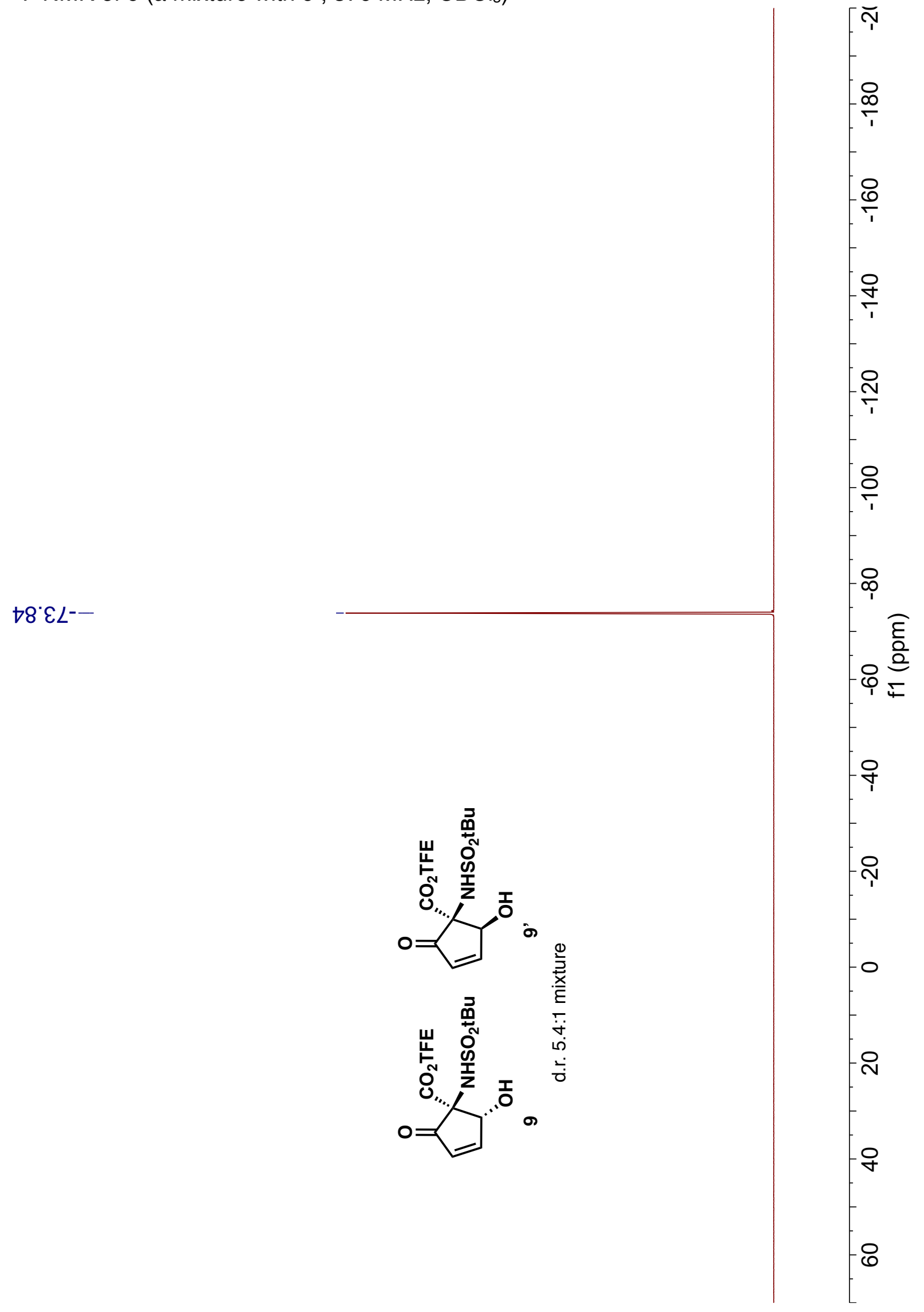


${ }^{1} \mathrm{H}$ NMR of $10\left(600 \mathrm{MHz}, \mathrm{CDCl}_{3}\right)$

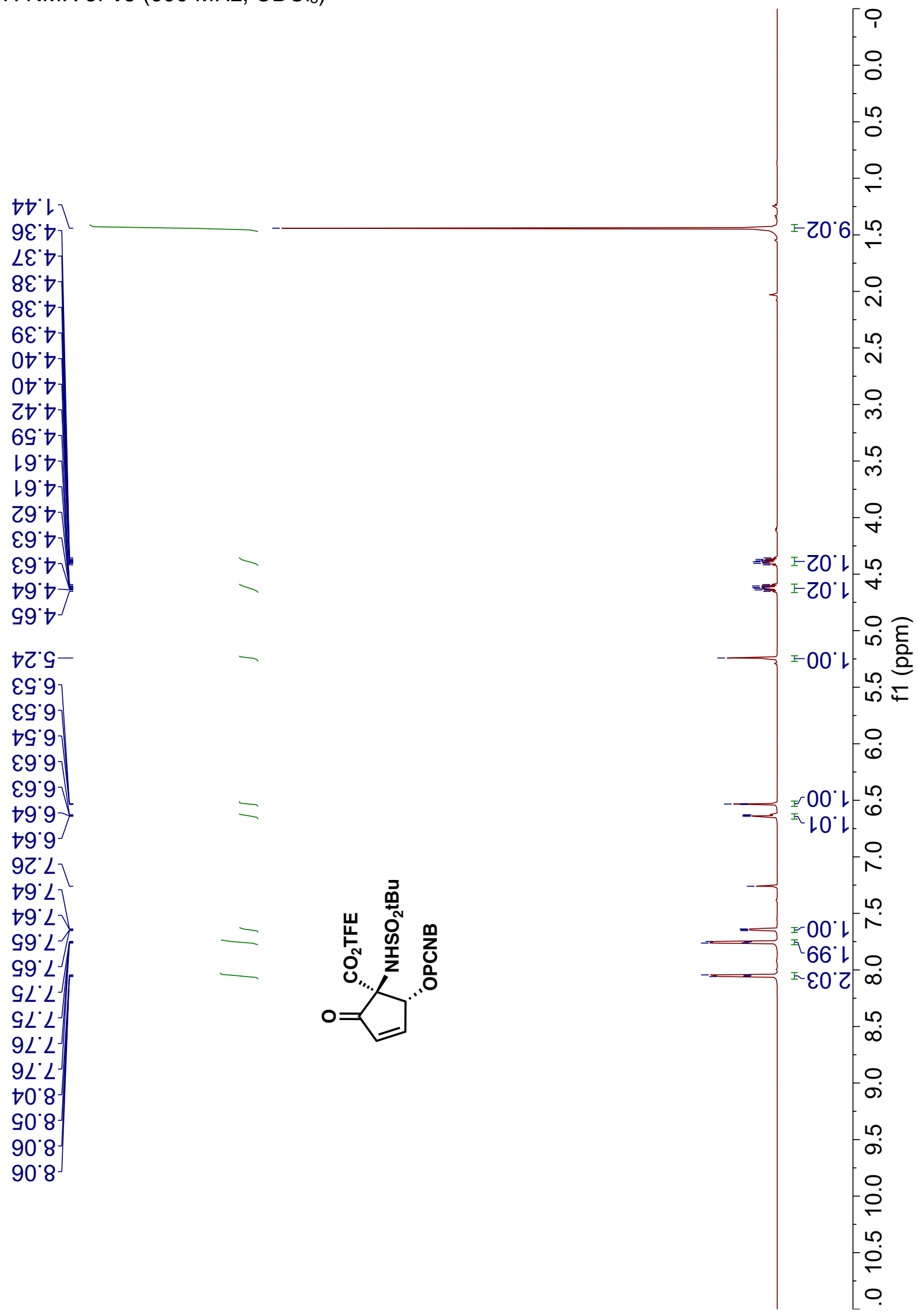


${ }^{13} \mathrm{C}$ NMR of $10\left(150 \mathrm{MHz}, \mathrm{CDCl}_{3}\right)$

$\varepsilon L^{\circ} \triangleright Z^{-}$

$6+19$

$28 \cdot 19$

$\angle 0 \cdot 29$

¿ะ'Z9

$\angle G^{\prime} Z 9$

ZI'ZL

$96.9 L$

$9+\angle L\}$

$\angle E^{\circ} \angle L$

19.08

$6 t^{\circ}<L$

$\varepsilon L \cdot \angle L$

เナ6เL

七てレL

80'๕Zレ

Z6'†ZL

เナ0EL

$\downarrow Z ' Z \varepsilon \downarrow$

$\nabla \varsigma^{\prime} Z \varepsilon \downarrow$

$0 L \cdot G \varepsilon$

98.9GL-

$6 L^{\circ}+9 t$

$\nabla S^{*} \triangleright 9 l$

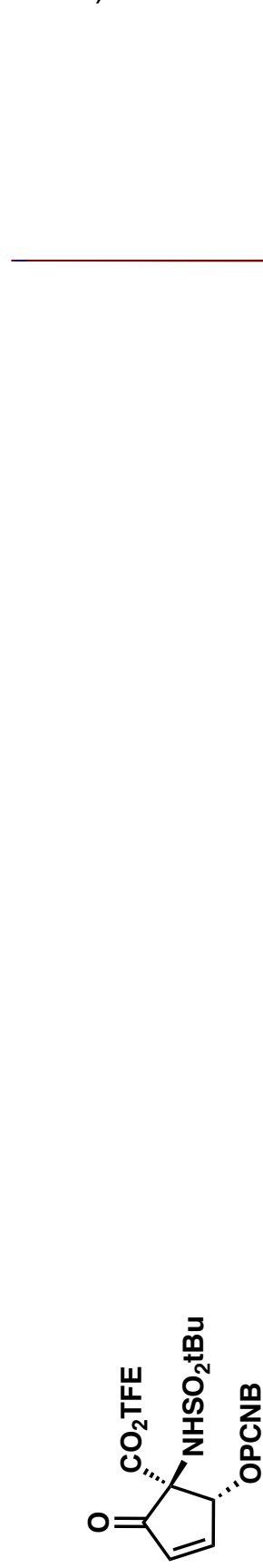

乙6'ย6เ-

$\mid \begin{aligned} & {[-0} \\ & -0 \\ & -\infty\end{aligned}$

우

m

-우

-

8

$-R$

$\infty$

요

응

음 응

$\div$

ิㅗㄴ

오

- 옹

용

$-8$

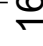

-온

$\stackrel{\infty}{\infty}$

욤

으

은

오 
${ }^{19} \mathrm{~F} \mathrm{NMR}$ of $10\left(376 \mathrm{MHz}, \mathrm{CDCl}_{3}\right)$

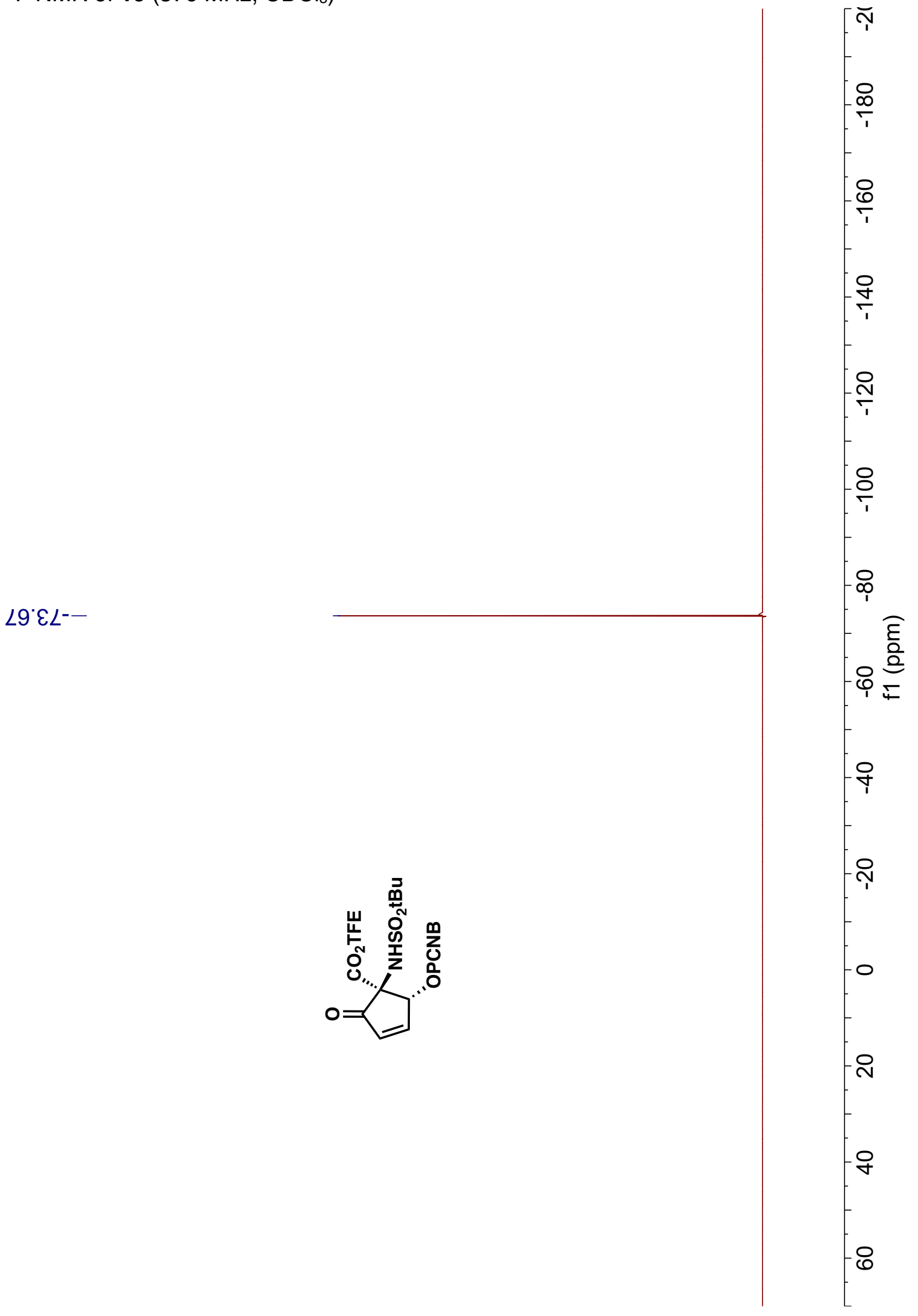


'H NMR of 10' (600 MHz, $\left.\mathrm{CDCl}_{3}\right)$

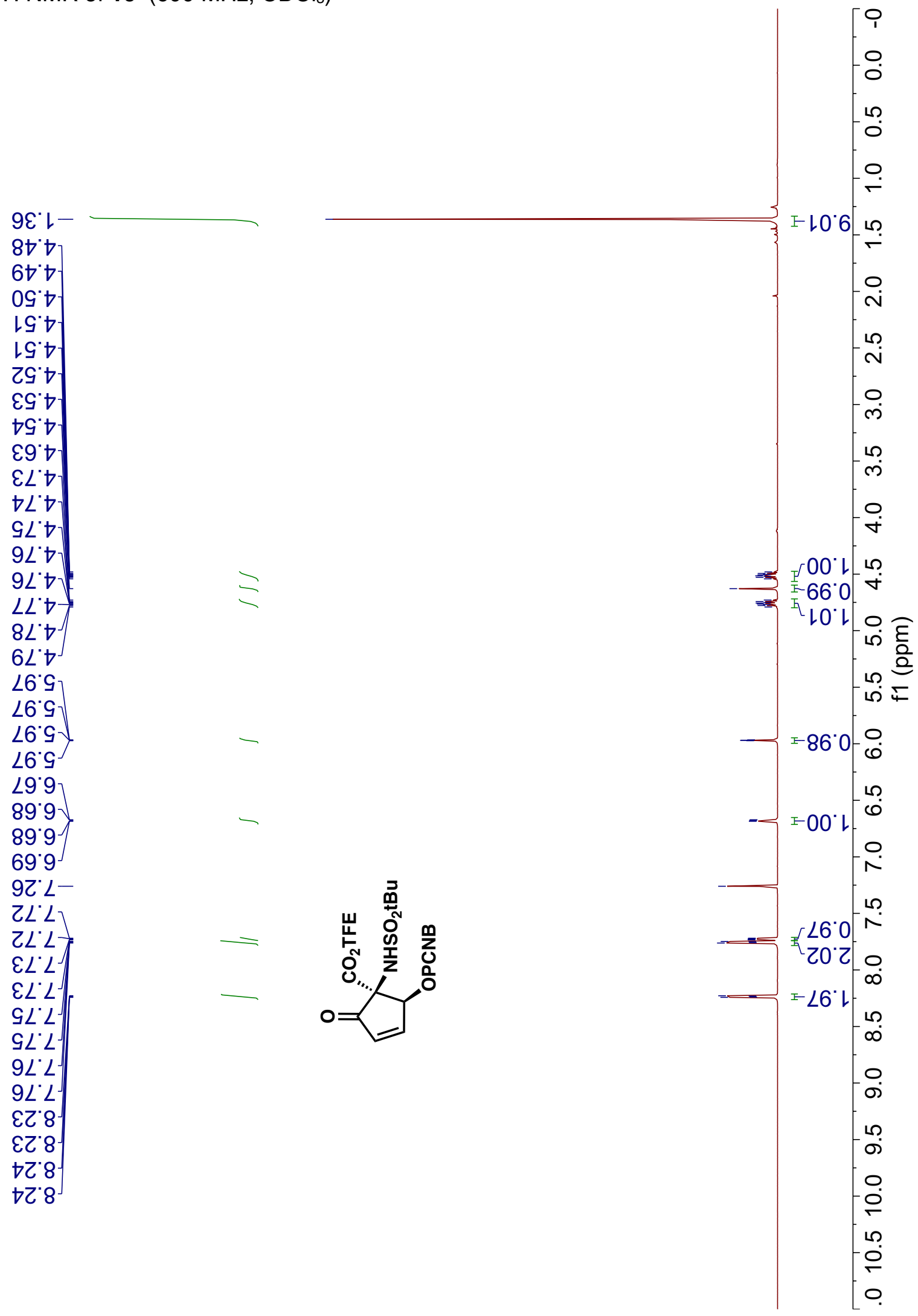


${ }^{13} \mathrm{C}$ NMR of $10^{\prime}\left(150 \mathrm{MHz}, \mathrm{CDCl}_{3}\right)$

$9 \varepsilon^{*} \triangleright Z^{-}$

$\angle \nabla^{\prime} \cdot 9$

$16.19]$

SL'Z9

$0 t^{\prime} Z 9-$

s9. 29

$19^{\circ} 69$

$8 S^{\circ} 9 L$

$96{ }^{\circ} 9 L^{\prime}$

$9 L^{\circ} \angle L$

$\angle E^{\prime} L L$

$\varepsilon \varepsilon^{\circ} \angle L$

เO 8 L

$\angle S^{\circ} 6 \mathrm{LL}$

Ot'เZL

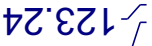

80.9ZL

LレLEL

$9 \varepsilon ' Z \varepsilon \downarrow$

$\angle \nabla \cdot Z \varepsilon L$

$\angle G^{\prime} 9 \varepsilon L$

06.991-

$\succ 8.991$

$8 l^{\circ} 99 l^{\prime}$

$\downarrow L ' t 6 レ-$

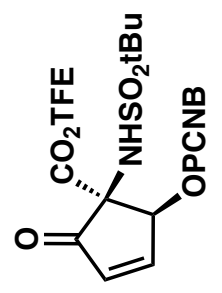

$\mid \begin{array}{ll}-0 \\ -0 \\ -0\end{array}$

요

요

우

is

8

-

$\infty$

요

음

을 응

$F$

은

ले

움

욤

8

$-5$

$\infty$

$\leftarrow$

웅

우

을 
${ }^{19}$ F NMR of 10' (376 MHz, $\mathrm{CDCl}_{3}$ )

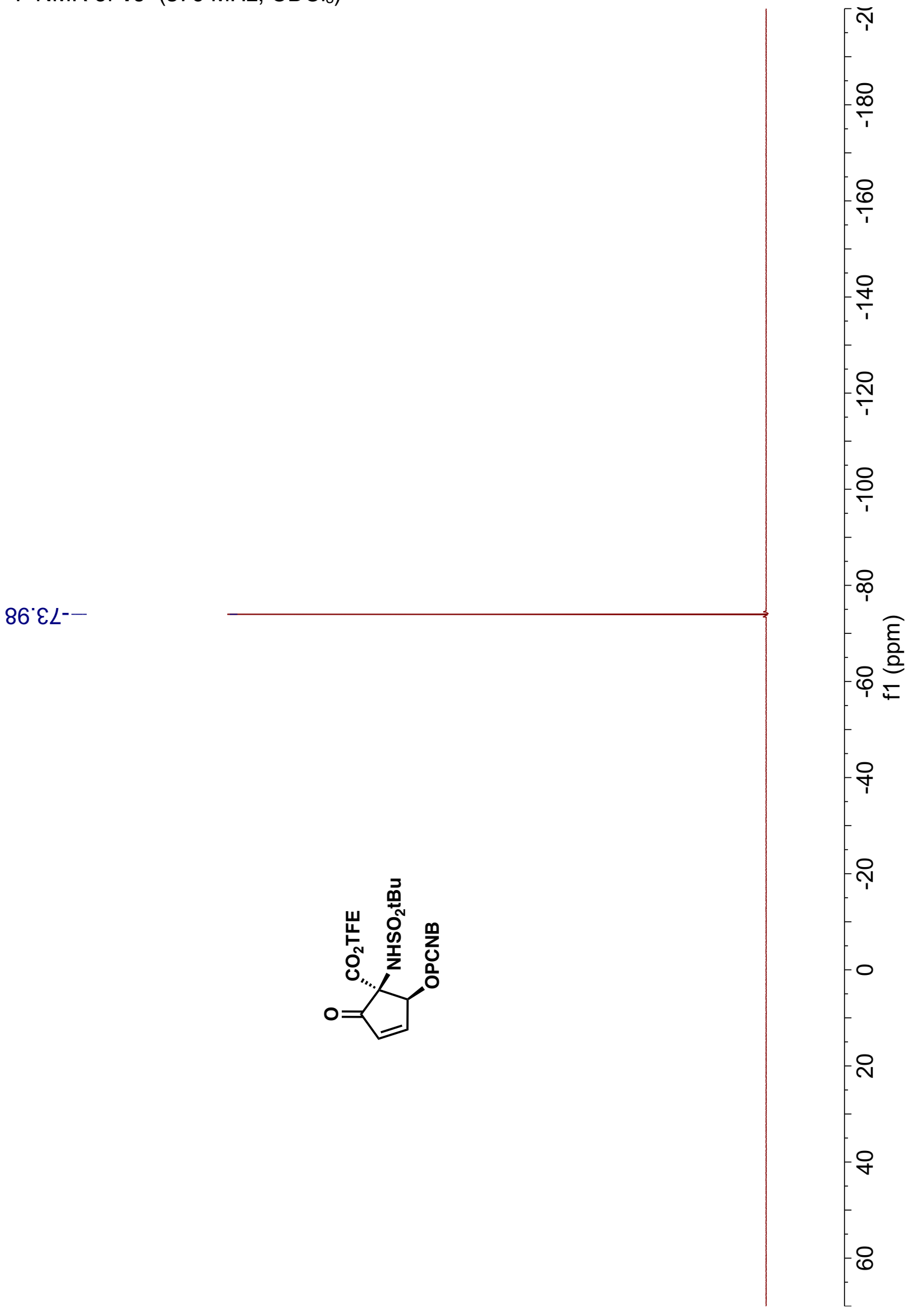


${ }^{1} \mathrm{H}$ NMR of $\mathbf{s} 1\left(600 \mathrm{MHz}, \mathrm{CDCl}_{3}\right)$

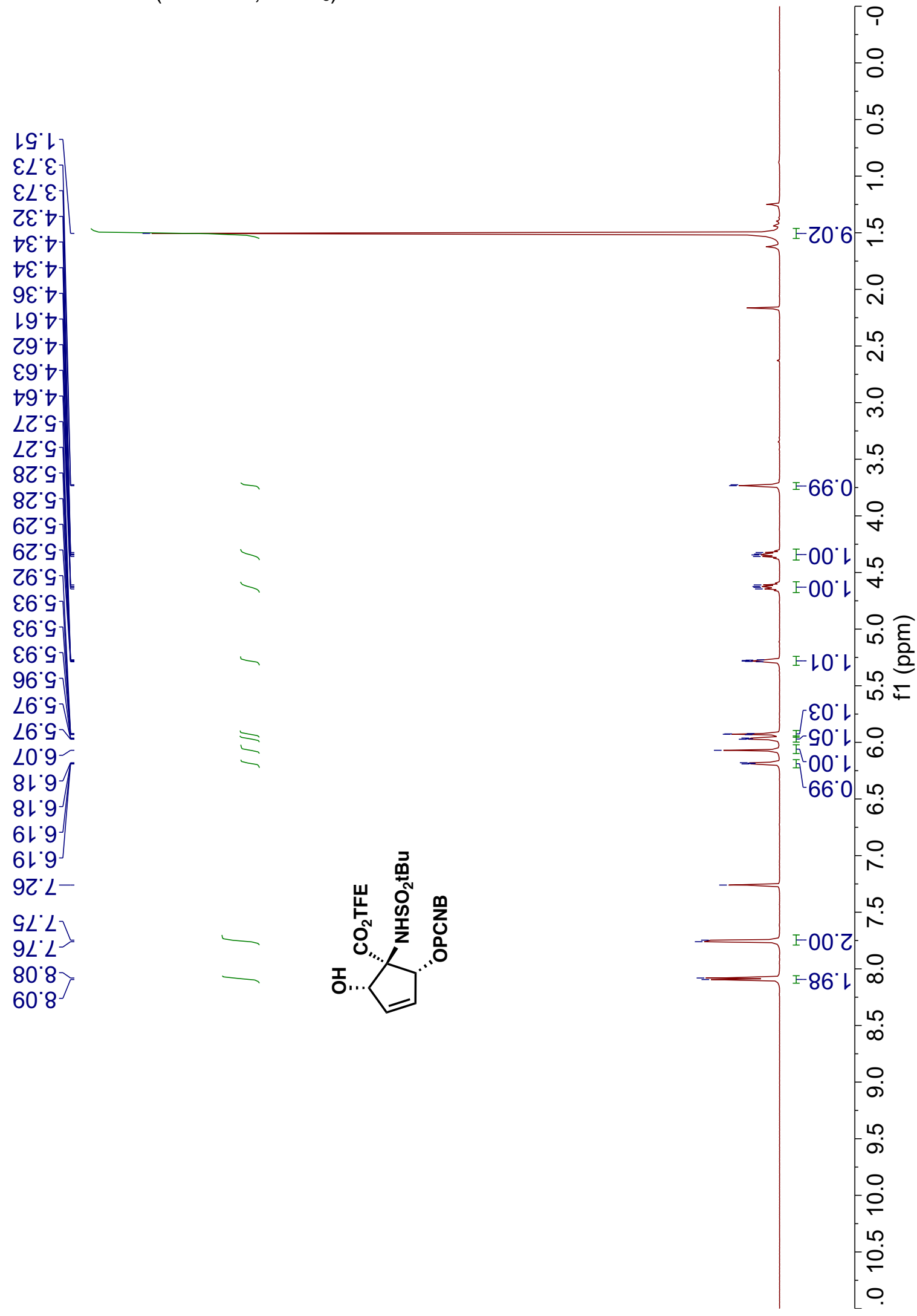


${ }^{13} \mathrm{C}$ NMR of $\mathbf{s} 1\left(150 \mathrm{MHz}, \mathrm{CDCl}_{3}\right)$

$8 \varepsilon^{\prime} \triangleright Z_{-}$

$9\llcorner 09$

70.19

$62 \cdot 19$

$\left.\varepsilon G^{\prime} 19\right\}$

$8 \angle \cdot 19$

$96.9 L$

$9 L^{\circ} \angle L \backslash$

$\angle E^{\prime} \angle L$

18.08

19.18

LL'98

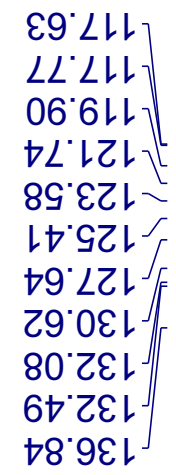

70.99 L $0 L^{\circ} \mathrm{LSI}^{\prime}$

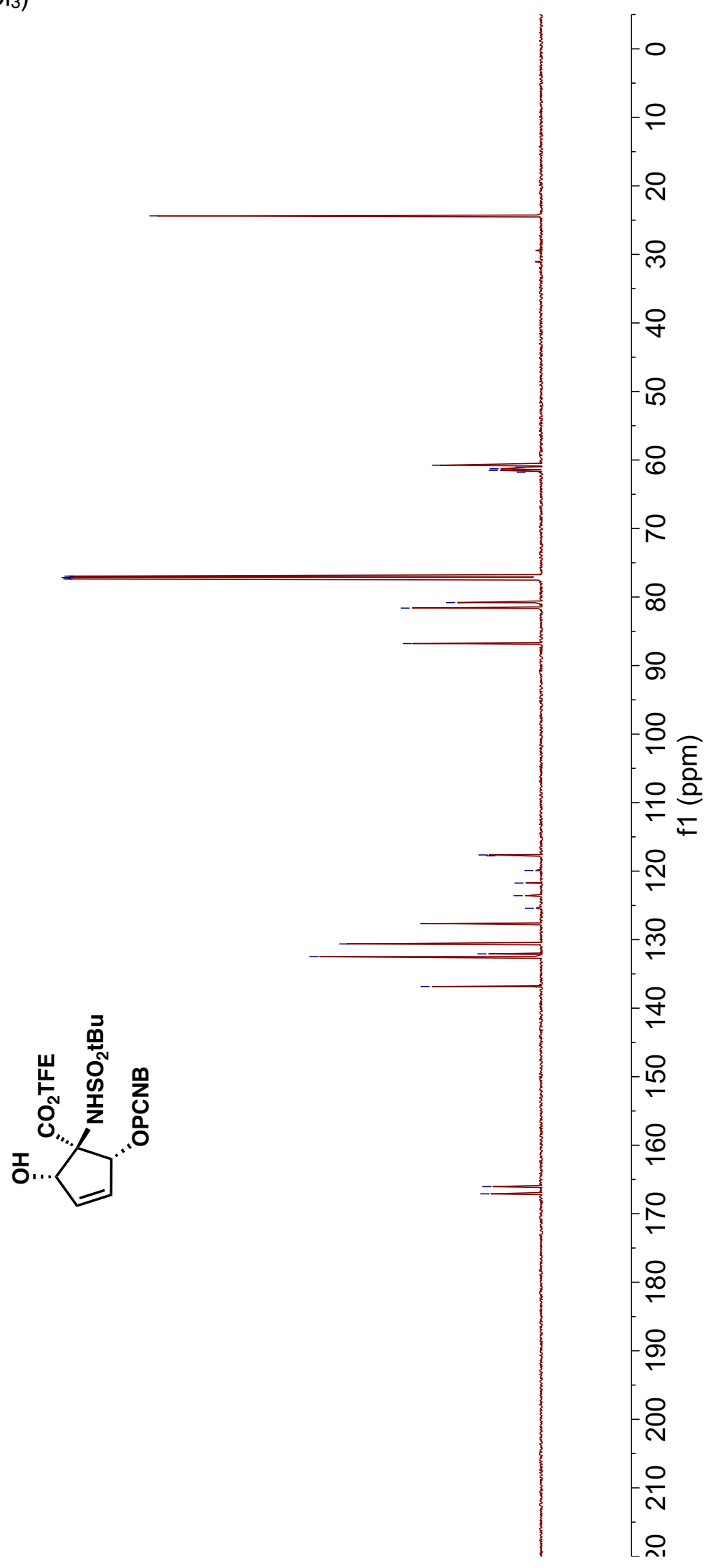


${ }^{19} \mathrm{~F} \mathrm{NMR}$ of $\mathbf{s} 1\left(376 \mathrm{MHz}, \mathrm{CDCl}_{3}\right)$

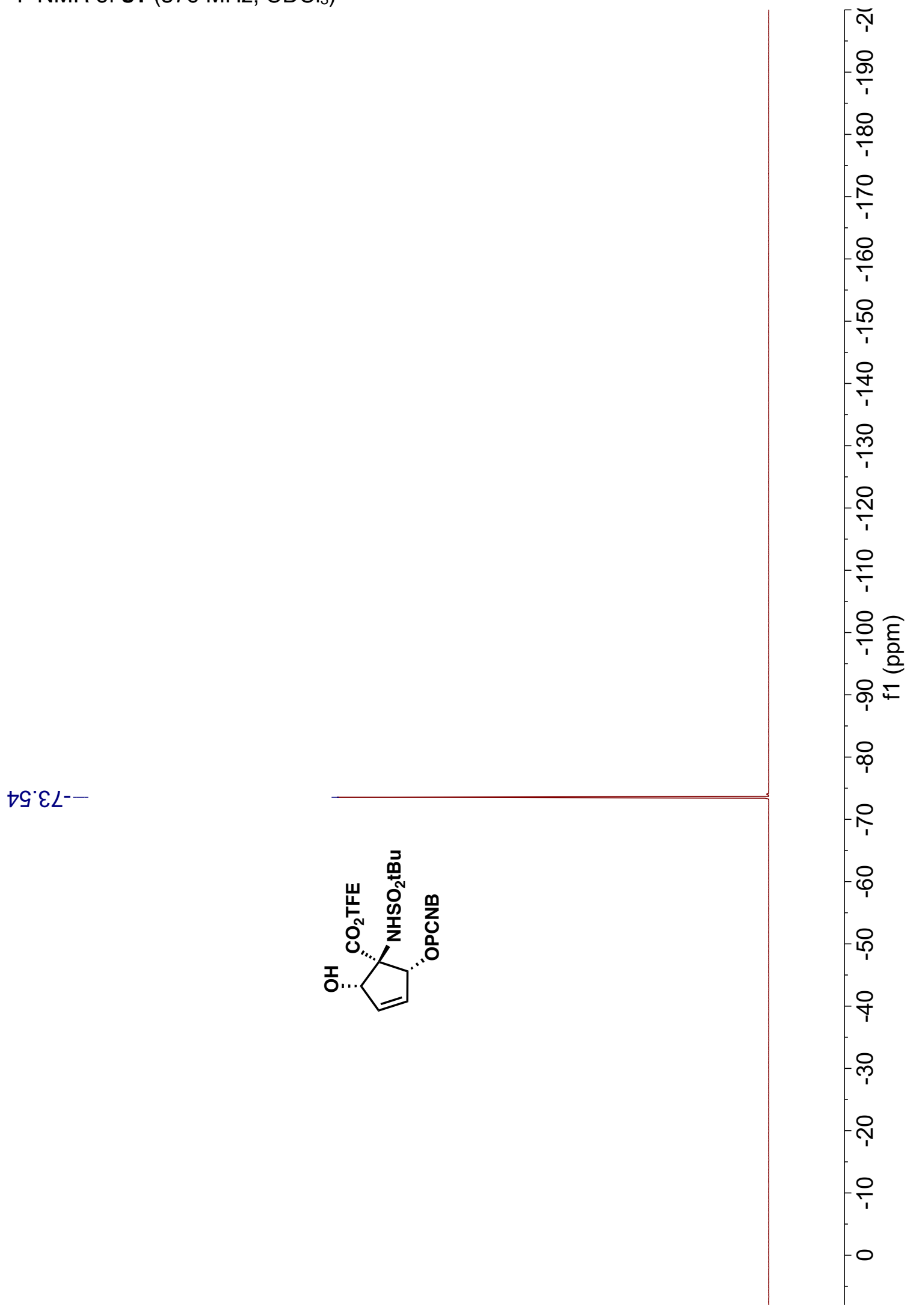




\section{HSQC of $\mathbf{s 1}$}

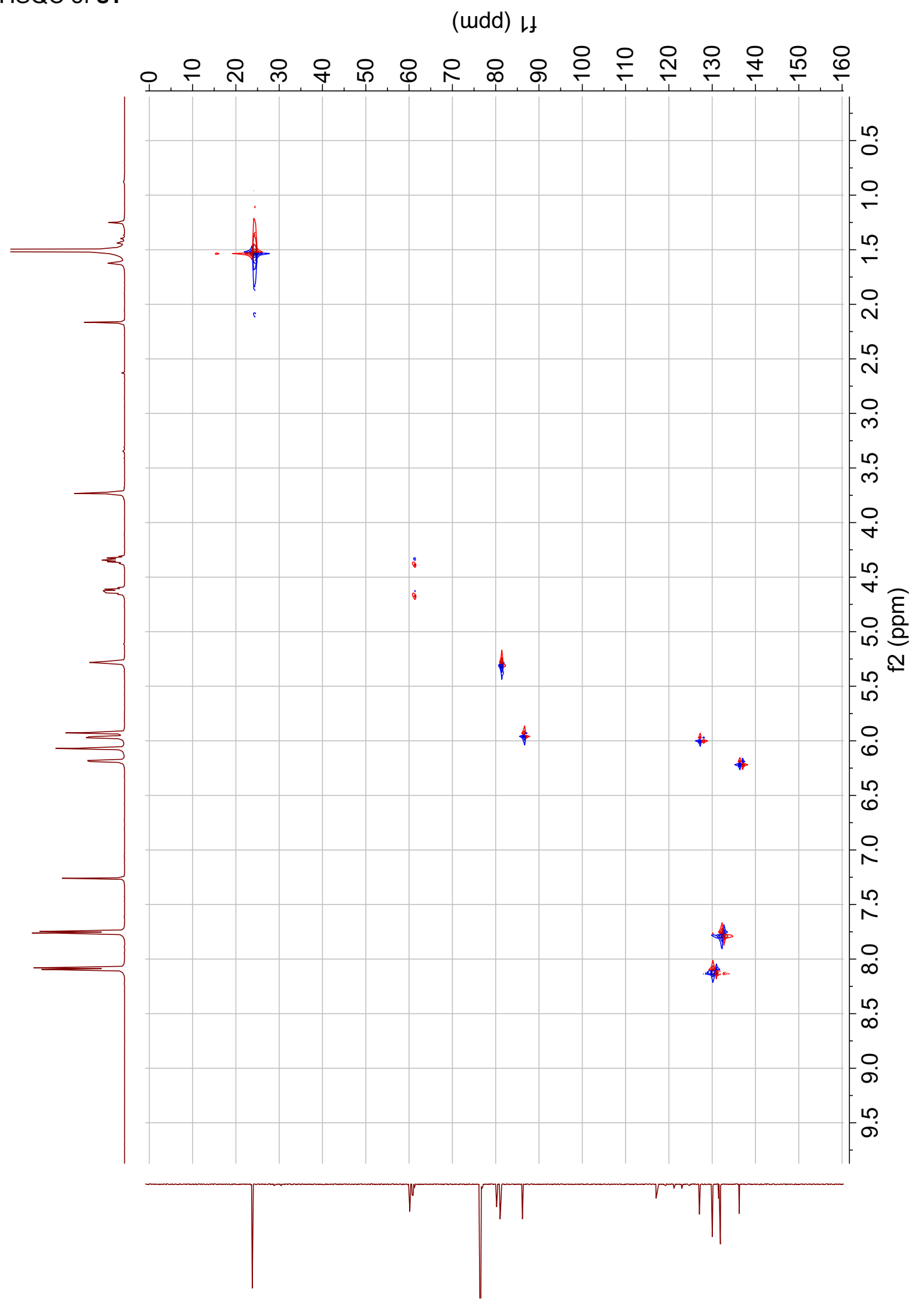




\section{COSY of $\mathbf{s 1}$}

(udd) If

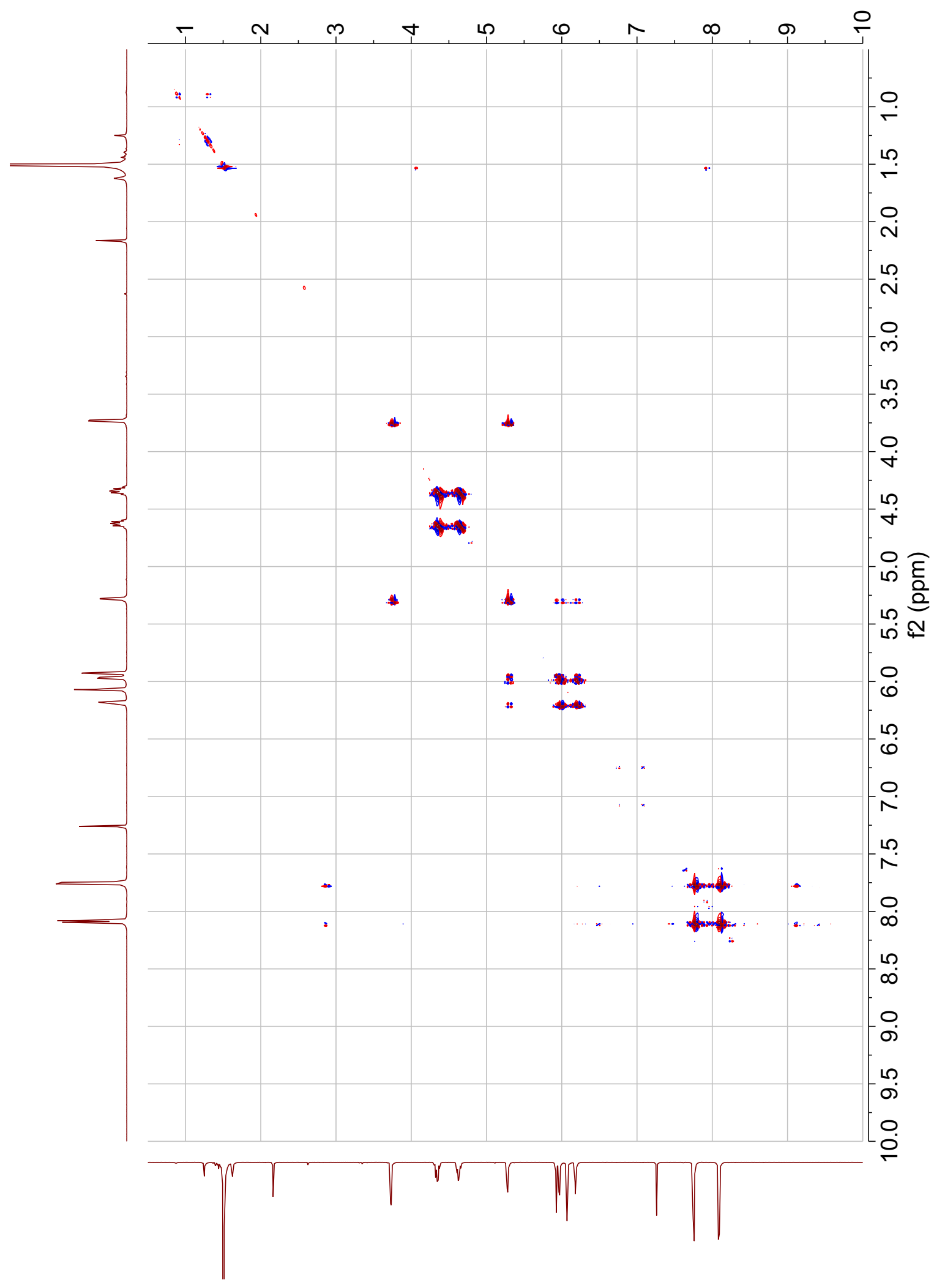


NOESY of $\mathbf{s} 1$

(udd) ᄂ

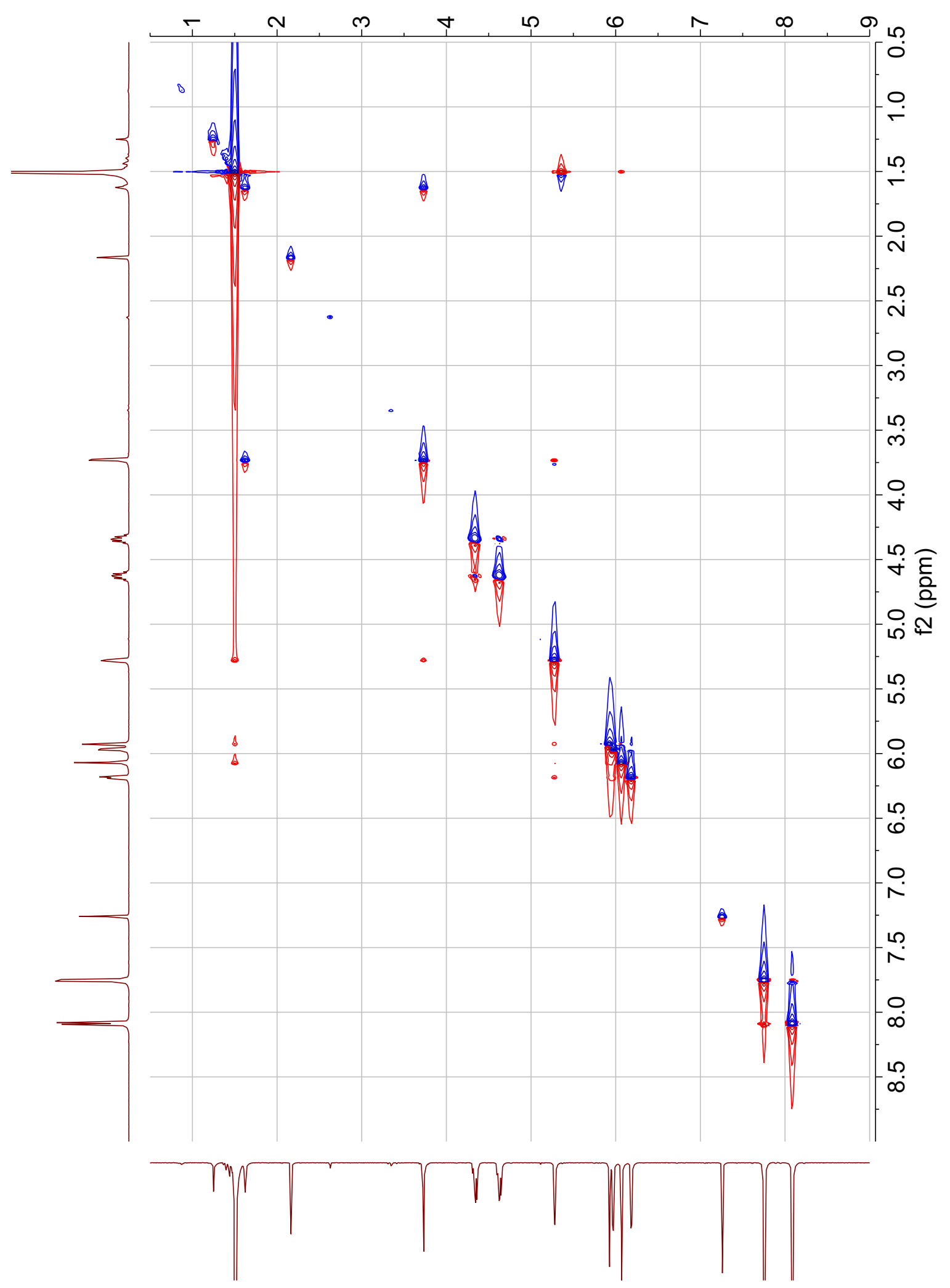


'H NMR of s1' (600 MHz, $\left.\mathrm{CDCl}_{3}\right)$

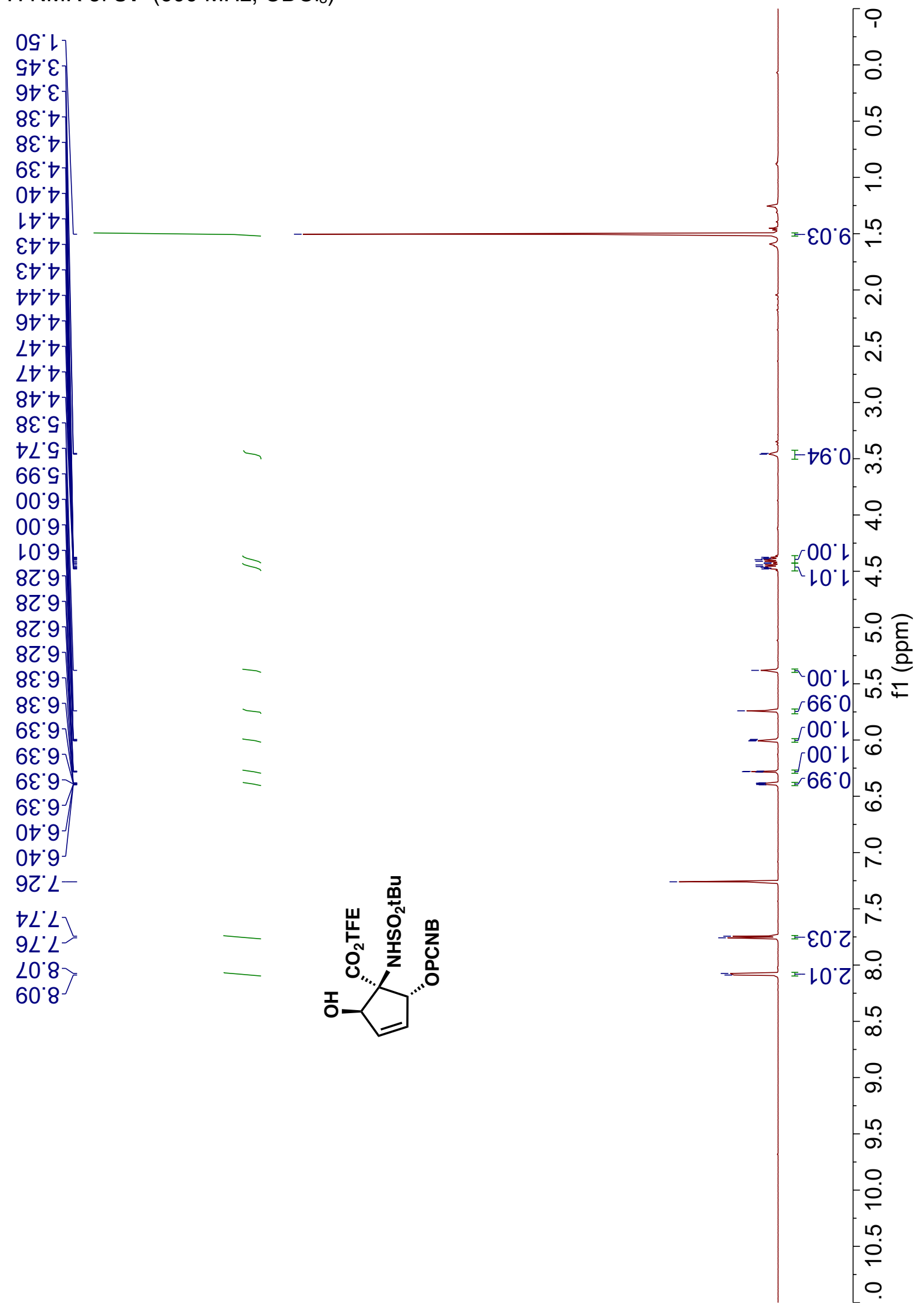


${ }^{13} \mathrm{C}$ NMR of $\mathbf{s} 1^{\prime}\left(150 \mathrm{MHz}, \mathrm{CDCl}_{3}\right)$

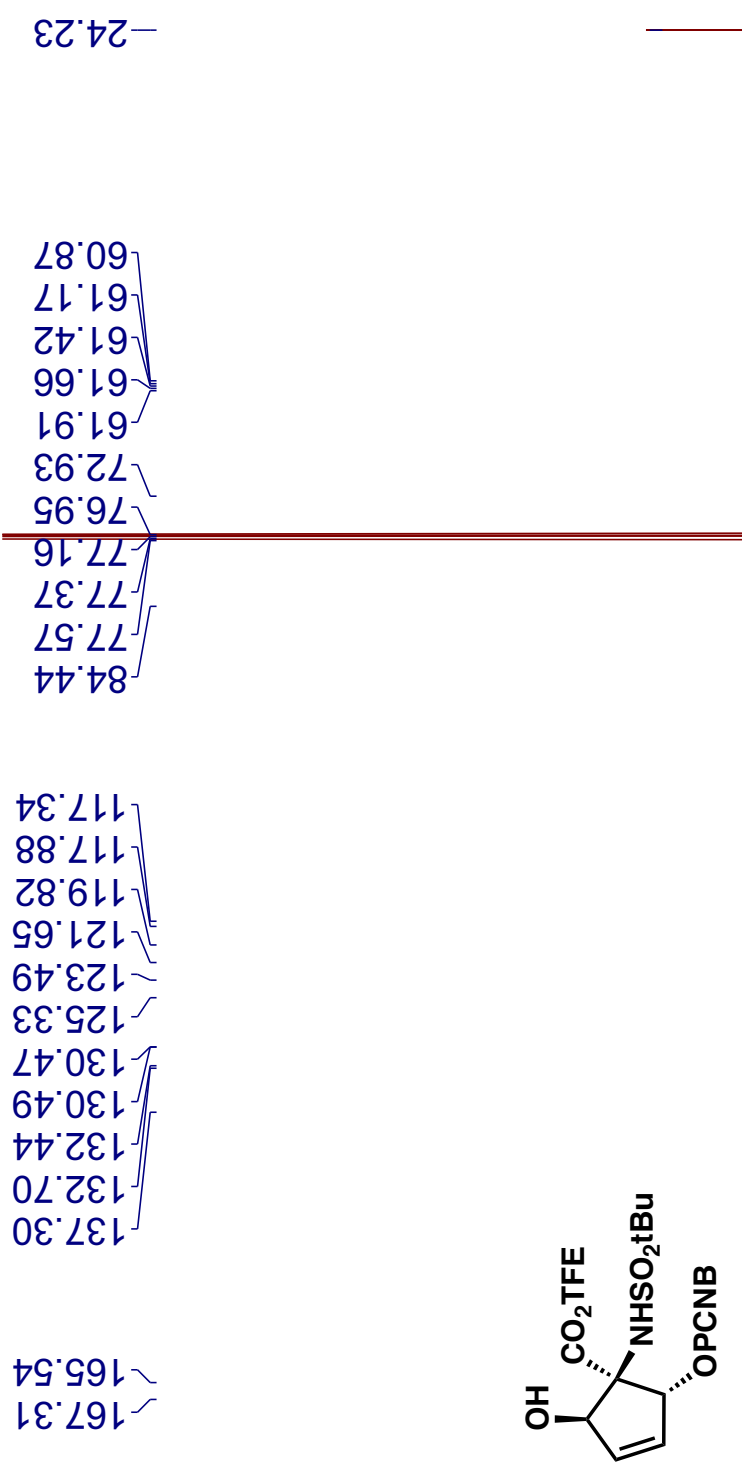

-0
-0
-0

요

우

웅

8

$-ᄋ$

$\infty$

চ

-ᄋ

으

으를 은

단

요

으

$\sim$

-옴

유

우

$-\frac{1}{2}$

$r$

$-\infty$

요

- 으

$-\frac{0}{\sqrt{2}}$

은 
${ }^{19}$ F NMR of s1' (376 MHz, $\mathrm{CDCl}_{3}$ )

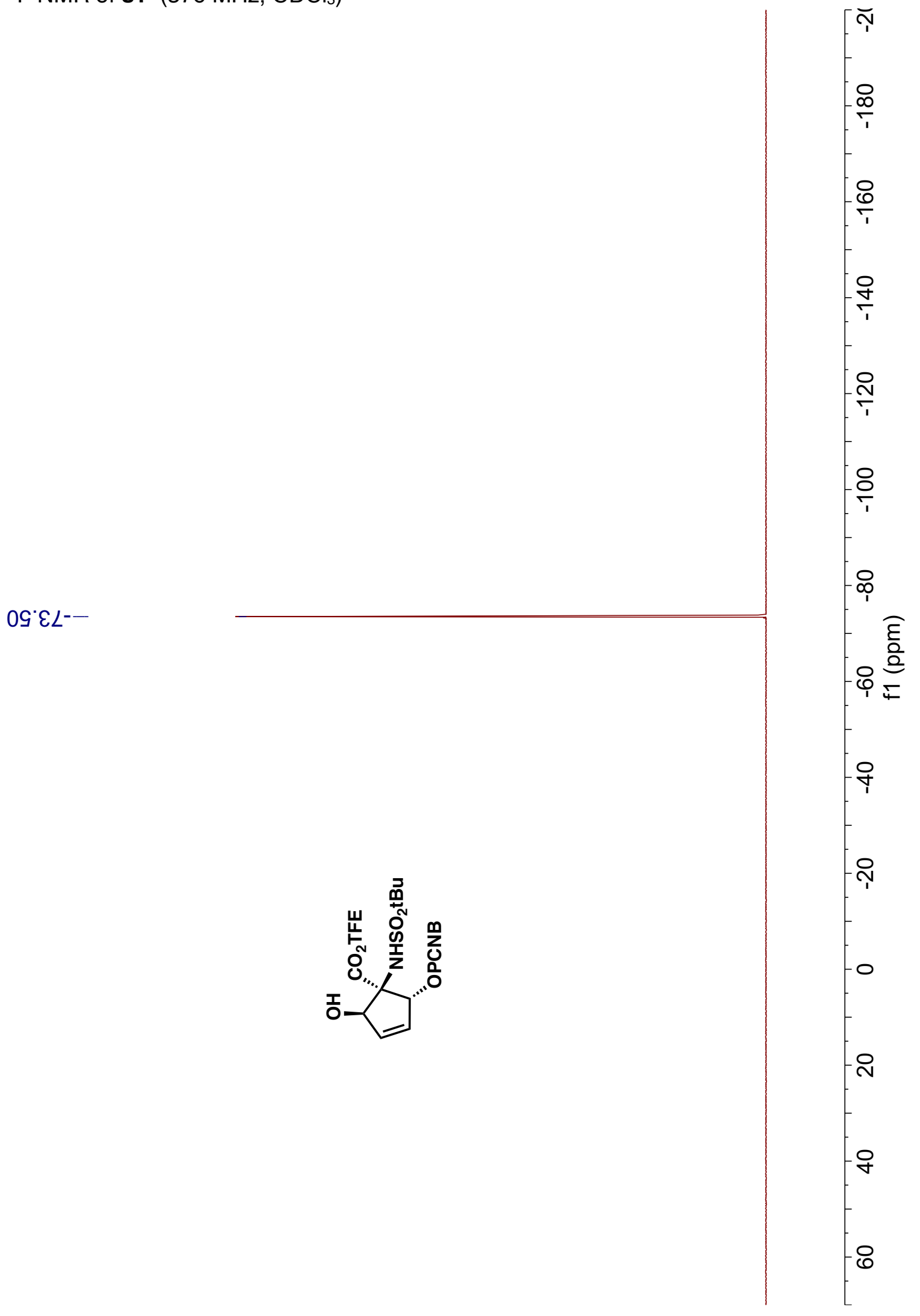


${ }^{1} \mathrm{H}$ NMR of $11\left(600 \mathrm{MHz}, \mathrm{CDCl}_{3}\right)$

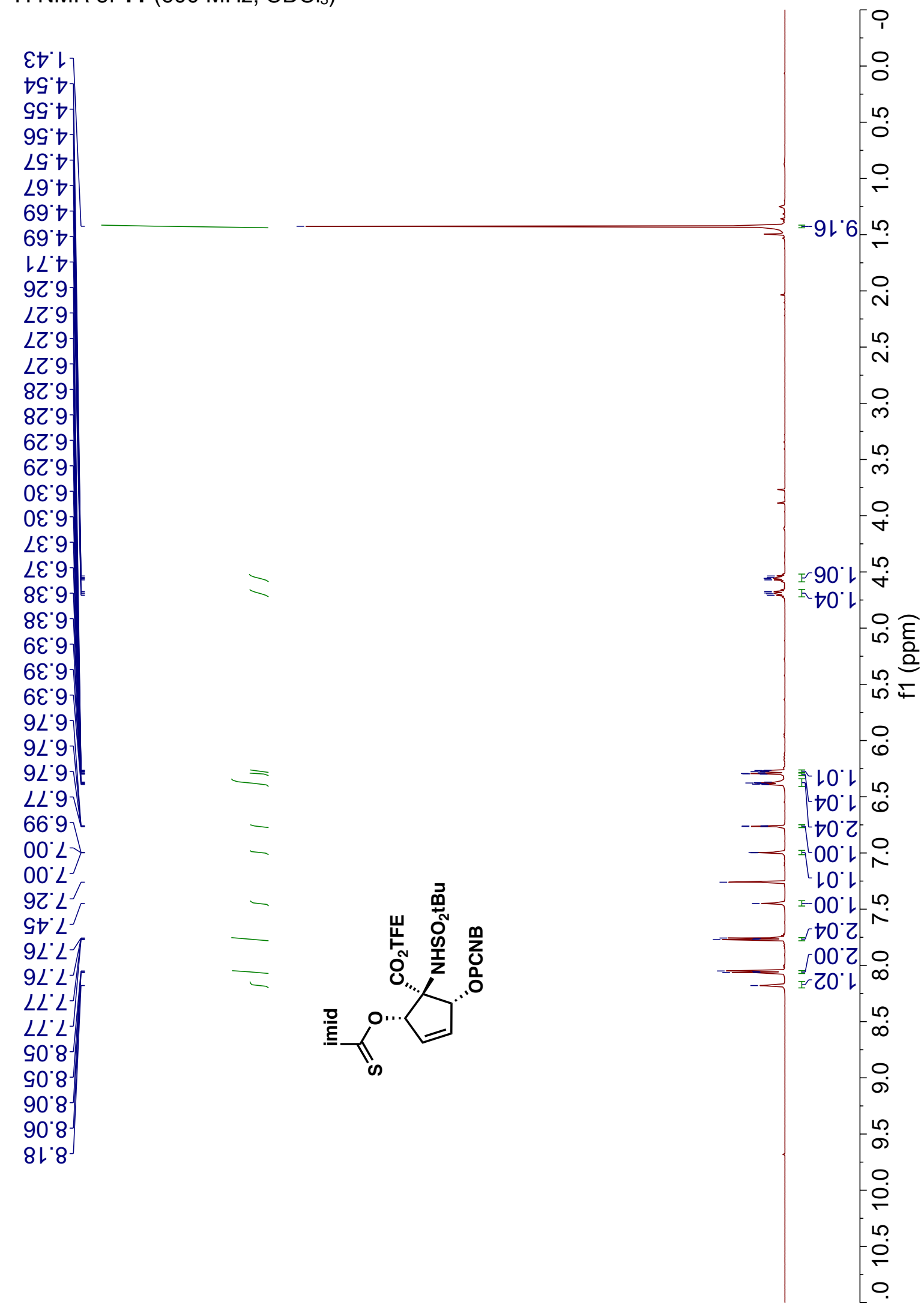


${ }^{13} \mathrm{C}$ NMR of $11\left(150 \mathrm{MHz}, \mathrm{CDCl}_{3}\right)$

$\varepsilon \iota^{\prime} \triangleright Z^{-}$

$\varepsilon 6.09$

$18 \cdot 19$

So. 29

$0 \varepsilon^{\prime} Z 9 \top$

G 'Z9

$99 \cdot 94$

96.92

$9 L^{\circ} \angle L$

$\angle E^{\prime} \angle L$

$89^{\circ} \varepsilon 8^{\circ}$

$\varepsilon Z^{\prime} 68$

$\angle G^{\prime} \angle L$

SL:LLL

GE'8L

$99^{\circ} 6 \mathrm{LL}$

$09^{\circ} L Z L$

†ย'ย乙レ

$\angle L G Z L-$

$\varepsilon t 0 \varepsilon L$

LILEL

GL'LEL

9Z'ZEL

GG'ZEL

$\angle G ' Z \varepsilon L$

$6 L^{\prime} 9 \varepsilon L$

$\varepsilon 6^{\circ}+9 \mathrm{~L}$

$6 l^{\cdot} 99 l^{-}$

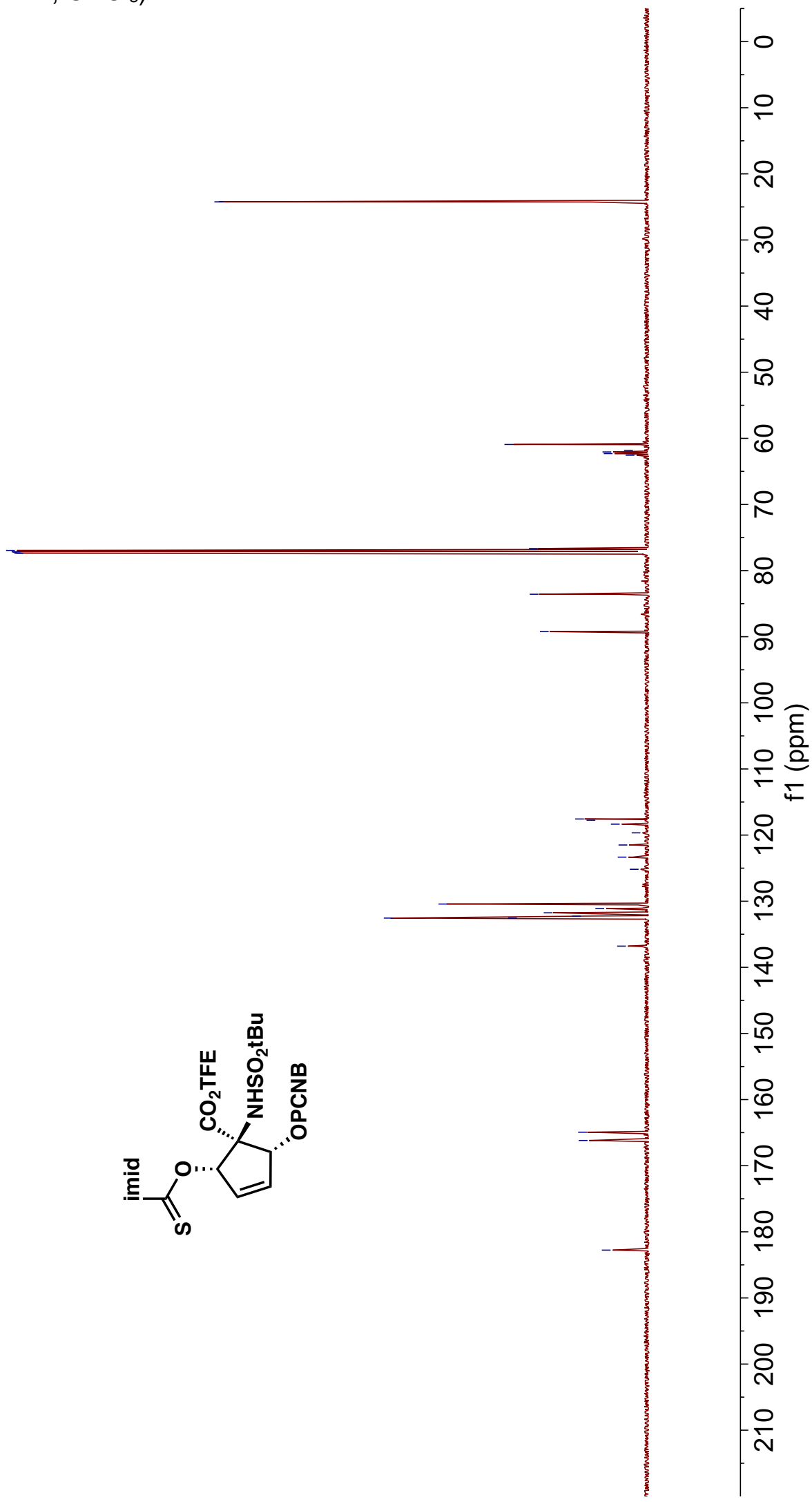

LL'Z8L- 
${ }^{19} \mathrm{~F} \mathrm{NMR}$ of $11\left(376 \mathrm{MHz}, \mathrm{CDCl}_{3}\right)$

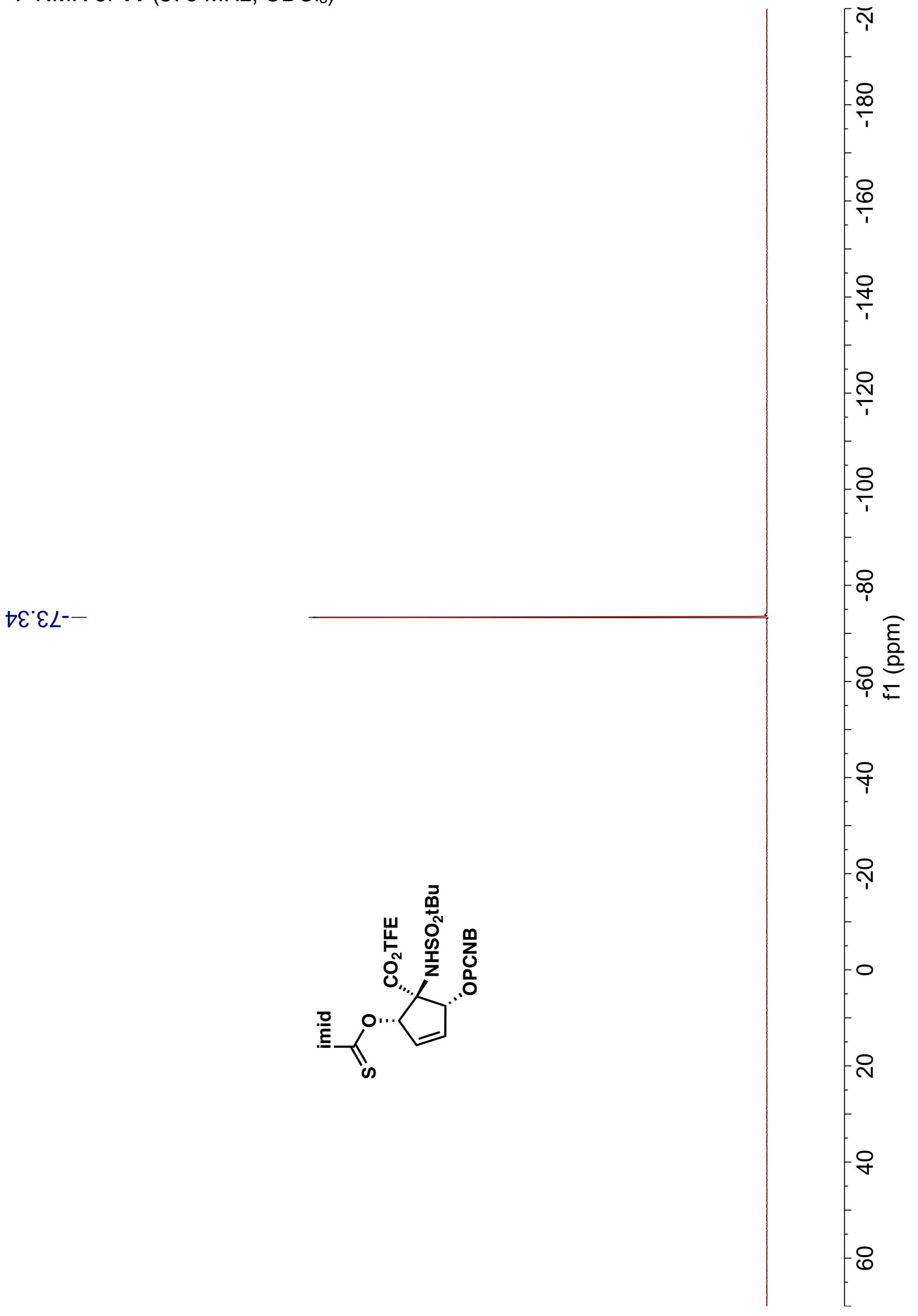


${ }^{1} \mathrm{H}$ NMR of $12\left(600 \mathrm{MHz}, \mathrm{CDCl}_{3}\right)$

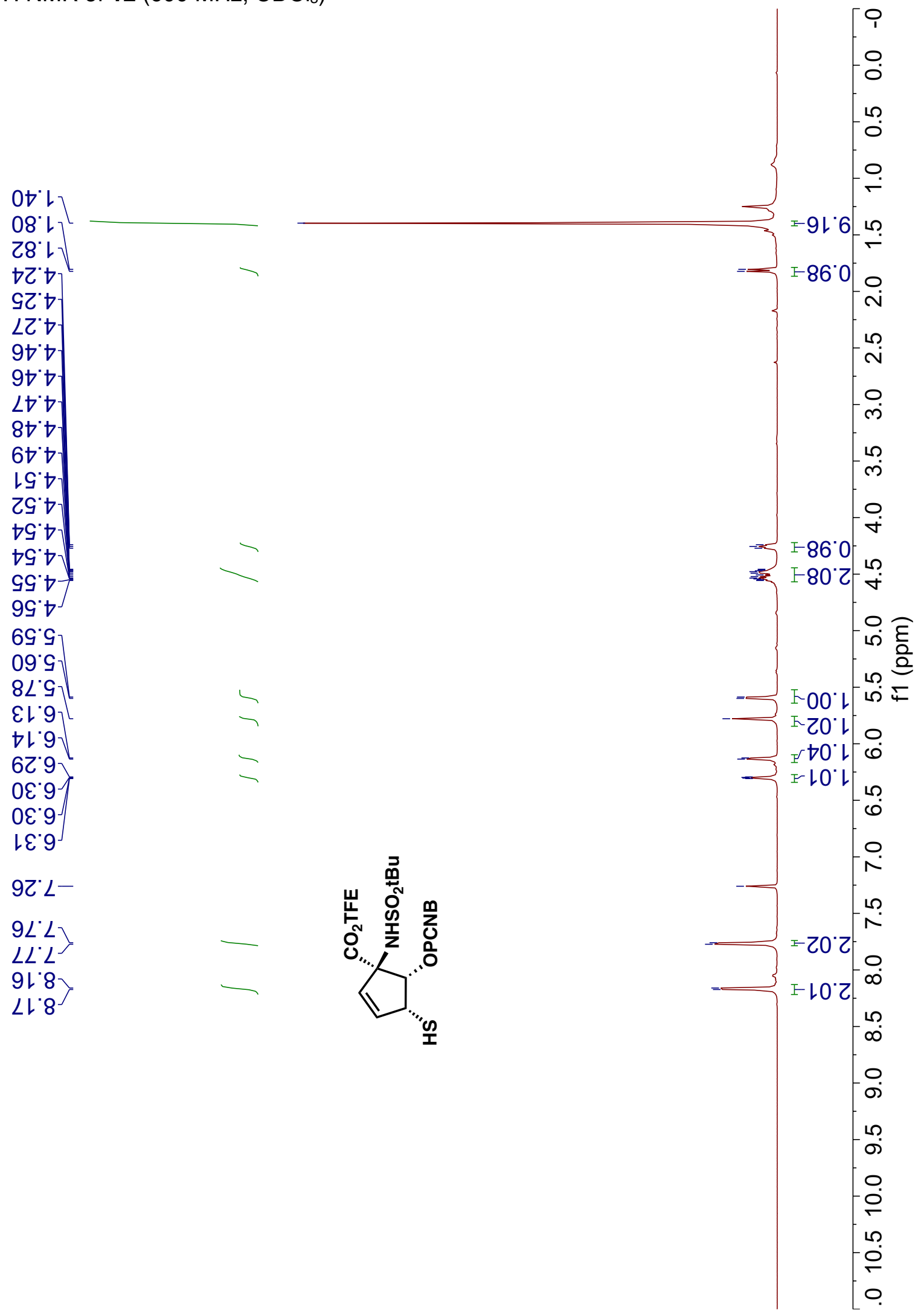


${ }^{13} \mathrm{C}$ NMR of $12\left(150 \mathrm{MHz}, \mathrm{CDCl}_{3}\right)$

$\angle Z^{*} \triangleright Z-$

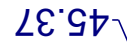

$\nabla Z^{\circ} 09$

$\varepsilon 9^{\circ} \cdot 9$

$88 \cdot 19$

乙L'Z9

$\angle \varepsilon \cdot 29$

$\nabla G^{\prime} \nabla L$

G6.9L

9L $L L$

$\angle E^{\circ} \angle L$

$8 \varepsilon^{\circ} 08$

GG'LルL

78 ㄴ

18.6L

G9เZL

8ナとてレ-

乙ย'Gてレ

98.8ZL-

99.0ع

$9 \varepsilon \cdot Z \varepsilon\llcorner$

$\varepsilon G^{\prime}$ ¿हL

$0 L \angle E L$

ルレ99レ-

งE'69l-

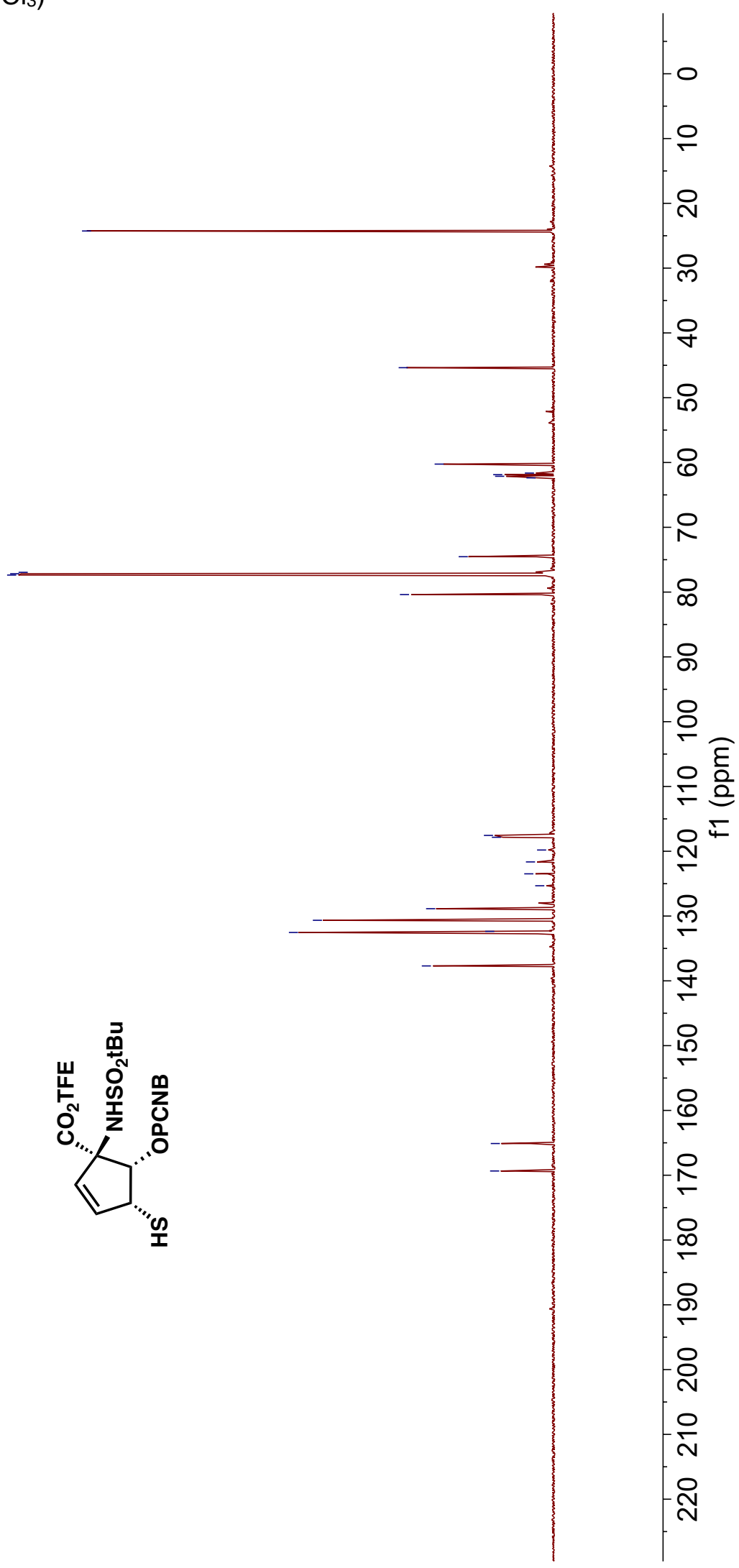


${ }^{19} \mathrm{~F} \mathrm{NMR}$ of $12\left(376 \mathrm{MHz}, \mathrm{CDCl}_{3}\right)$

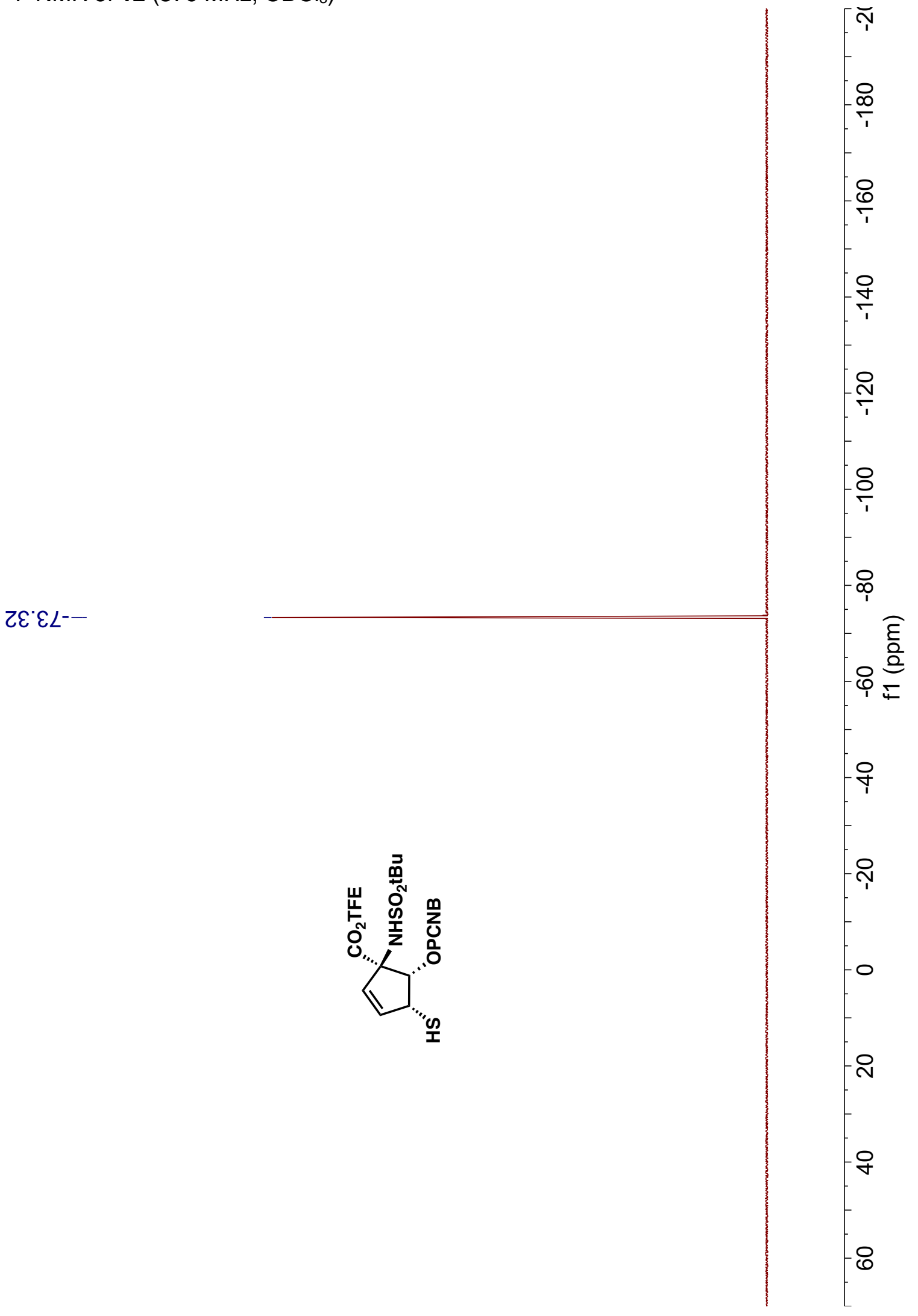


${ }^{1} \mathrm{H}$ NMR of $13\left(600 \mathrm{MHz}, \mathrm{CDCl}_{3}\right)$

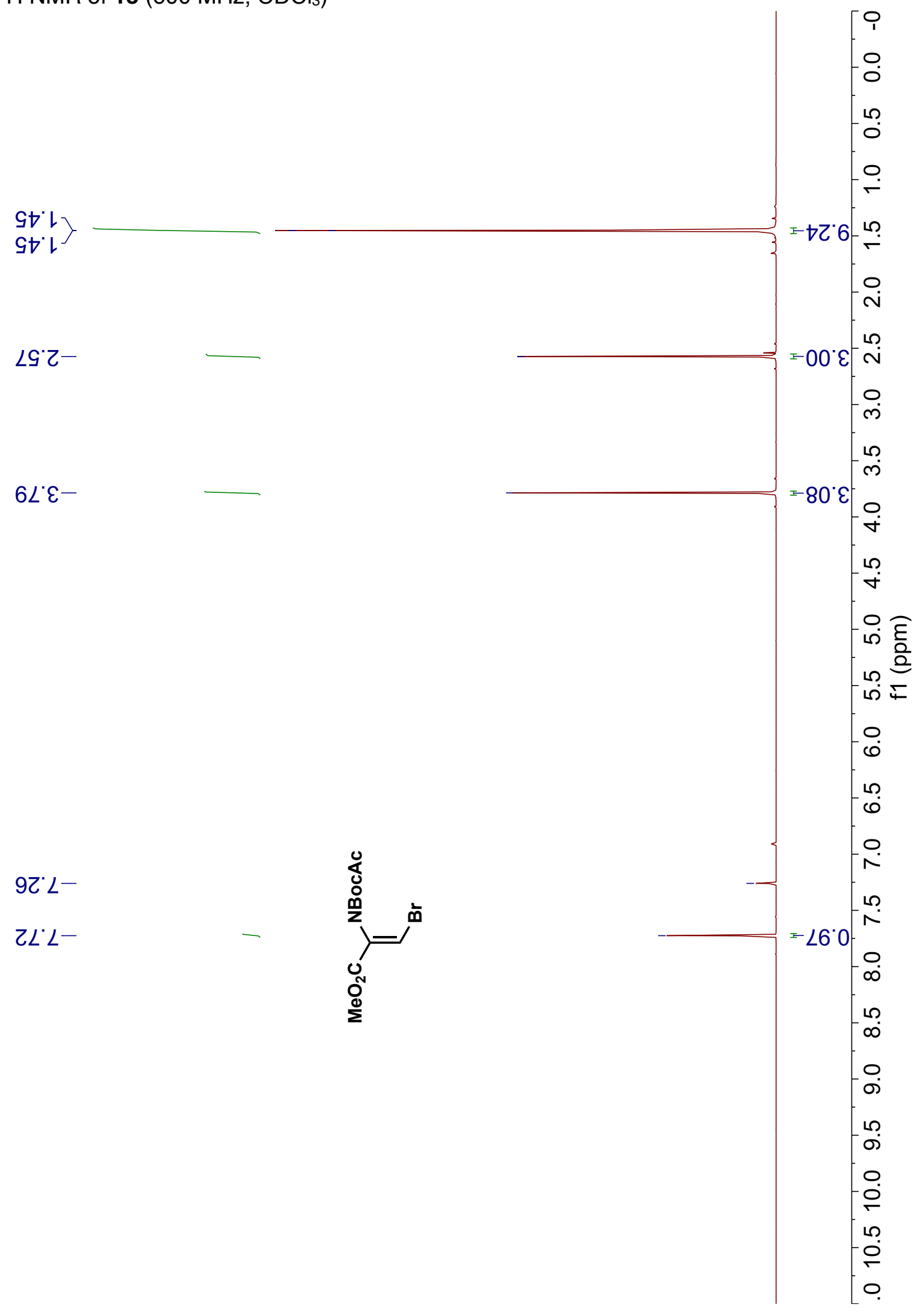


${ }^{13} \mathrm{C} \mathrm{NMR}$ of $13\left(150 \mathrm{MHz}, \mathrm{CDCl}_{3}\right)$

$98^{\circ} \mathrm{G} 乙$

$98^{\circ} L^{-}$

Z6'Z9-

96.9L

$9 L^{\circ} L L Y$

$\angle E^{\prime} \angle L$

$91 \div 8$

ヤ0๋てレー

৪乙`†とレ-

Sガ0งレー

06เงレー

டナ゙レレレー
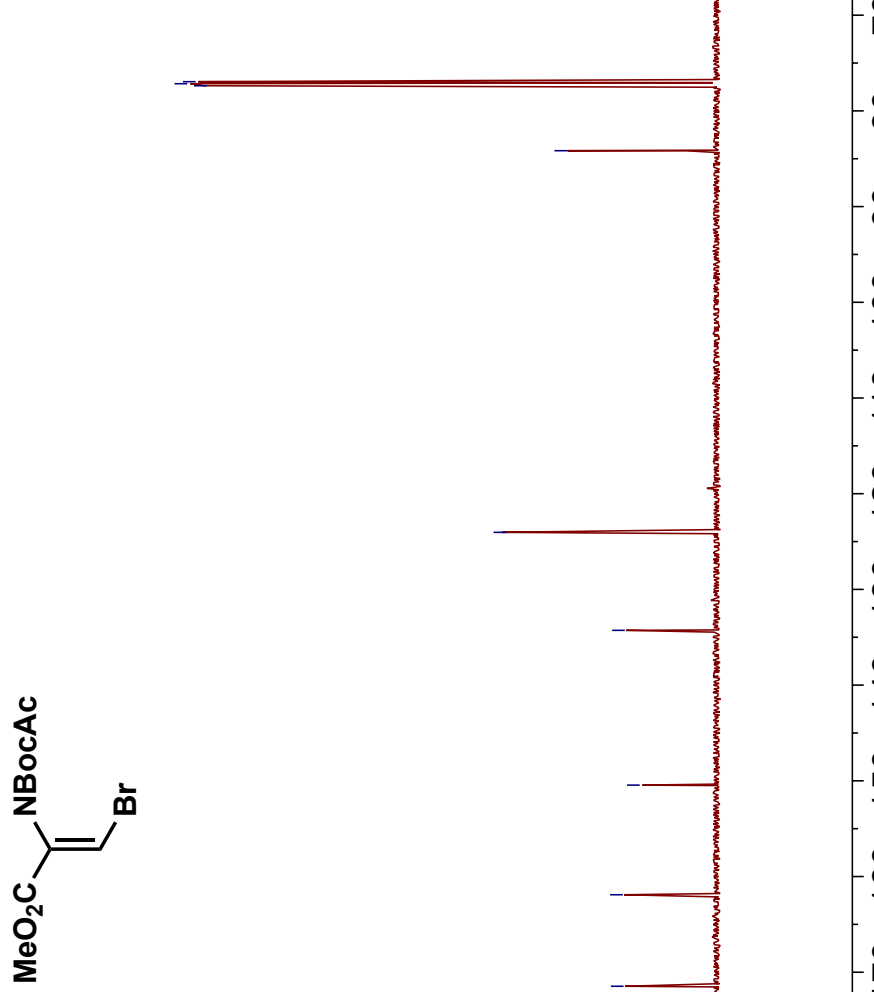

-

$-\frac{}{\div} \frac{\mathrm{g}}{0}$

읃 을

오

N

$\stackrel{m}{\stackrel{m}{2}}$

$-\frac{1}{2}$

$-10$

$-8$

$-\frac{1}{0}$

$-\infty$

요

유

$-\frac{0}{N}$

요 


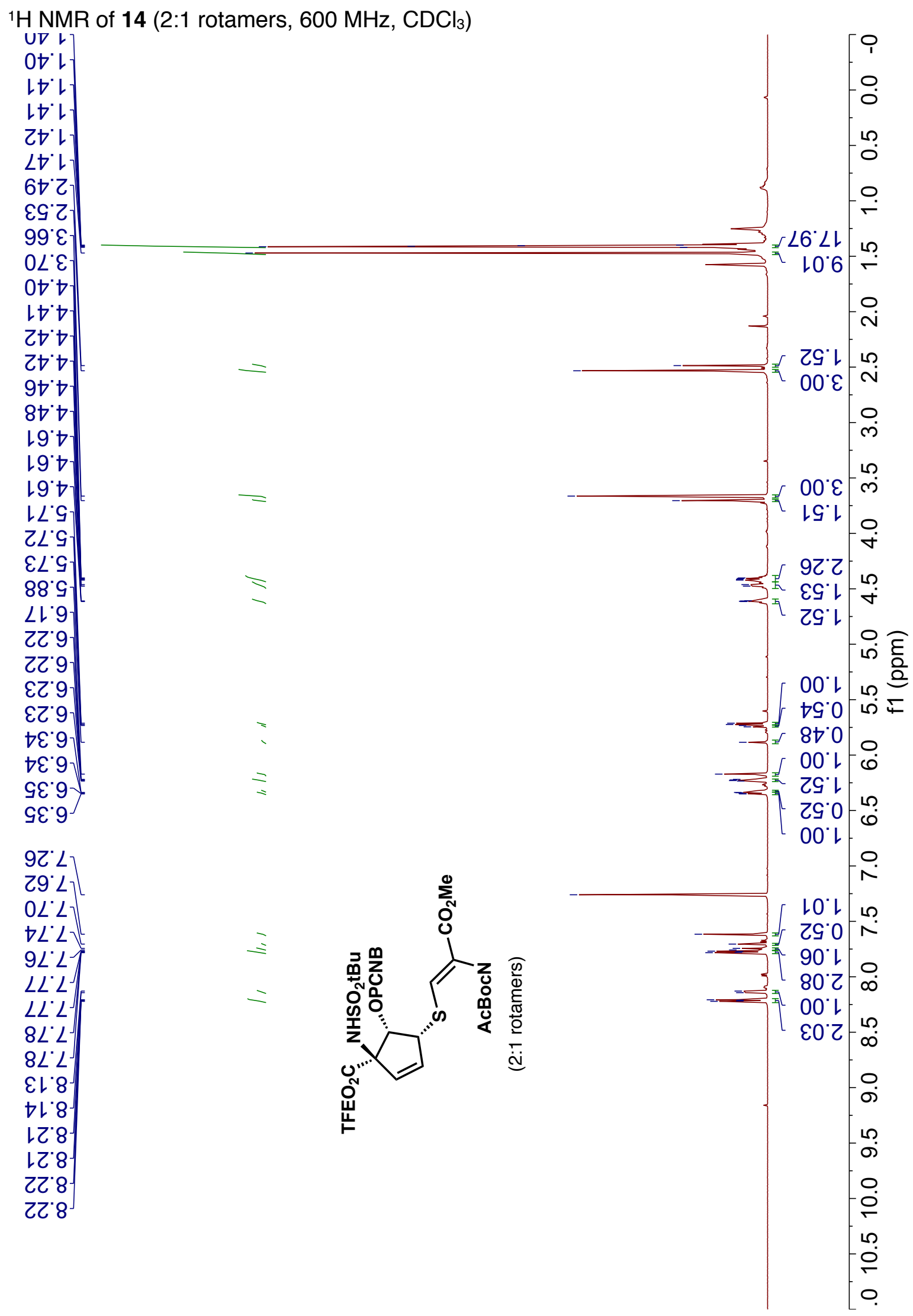


${ }^{13} \mathrm{C}$ NMR of 14 (2:1 rotamers, $\left.150 \mathrm{MHz}, \mathrm{CDCl}_{3}\right)$

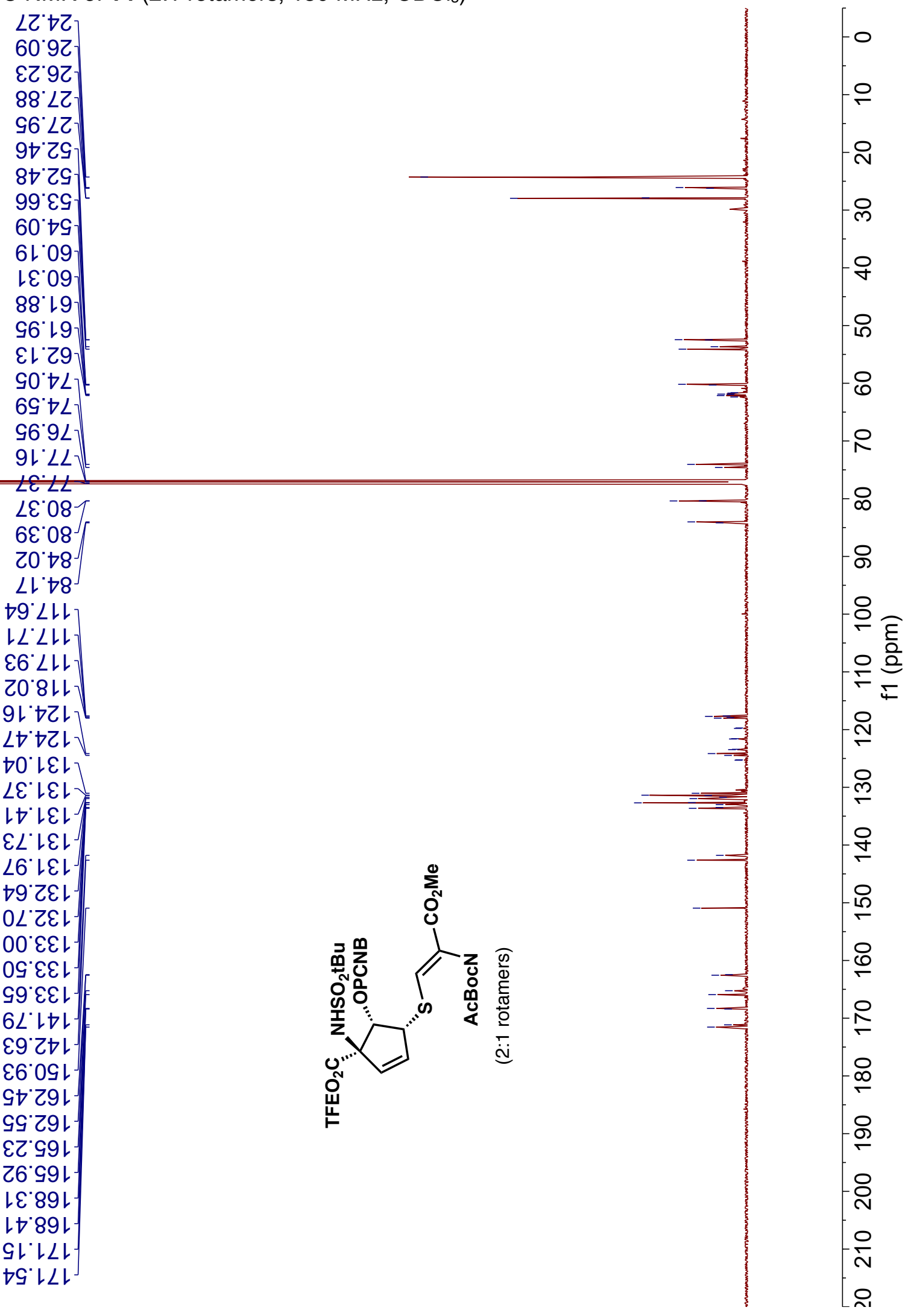


${ }^{19} \mathrm{~F} \mathrm{NMR}$ of $14\left(2: 1\right.$ rotamers, $\left.376 \mathrm{MHz}, \mathrm{CDCl}_{3}\right)$

9L'EL-

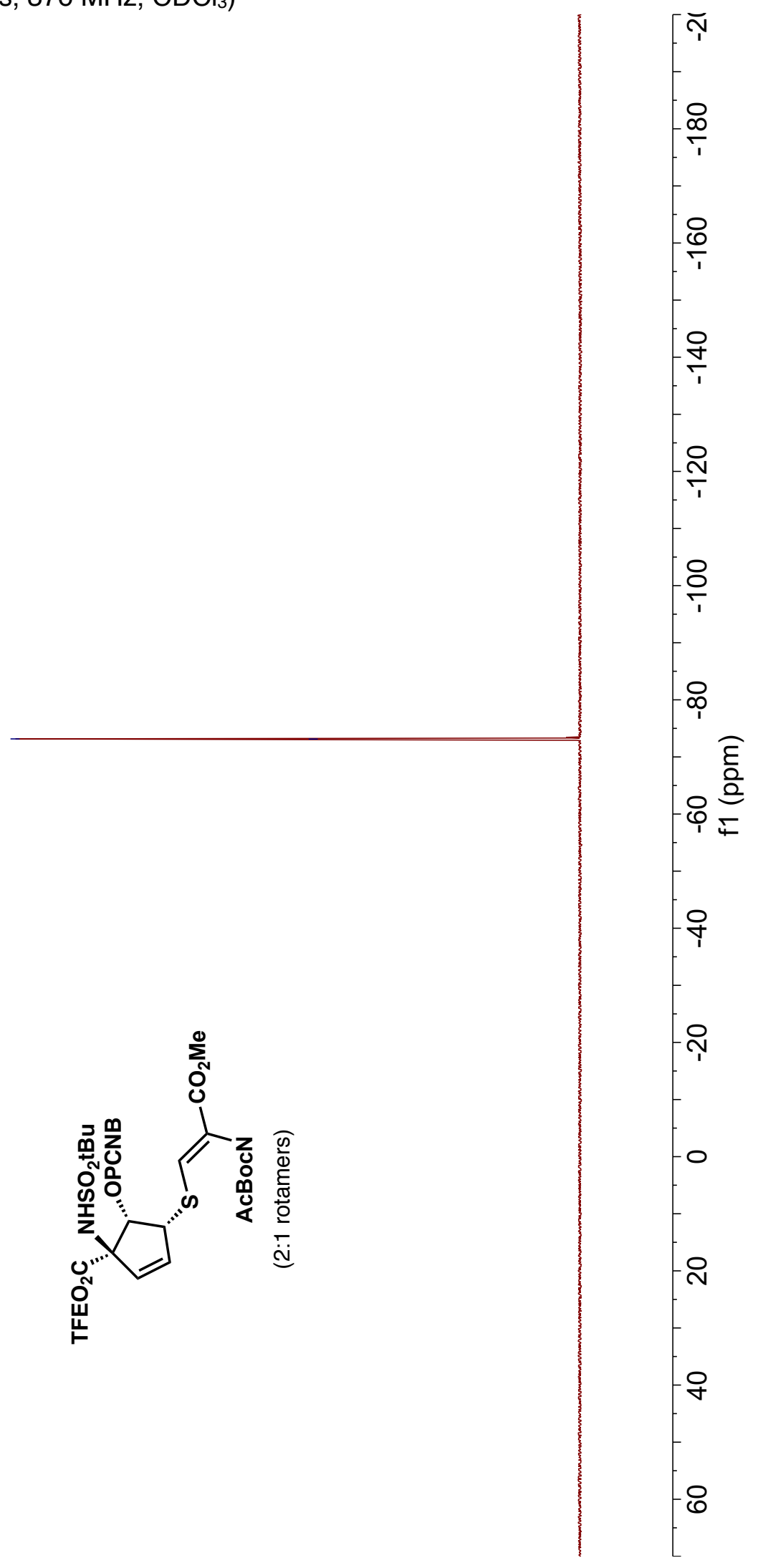


${ }^{1} \mathrm{H}$ NMR of $15\left(600 \mathrm{MHz}, \mathrm{CDCl}_{3}\right)$
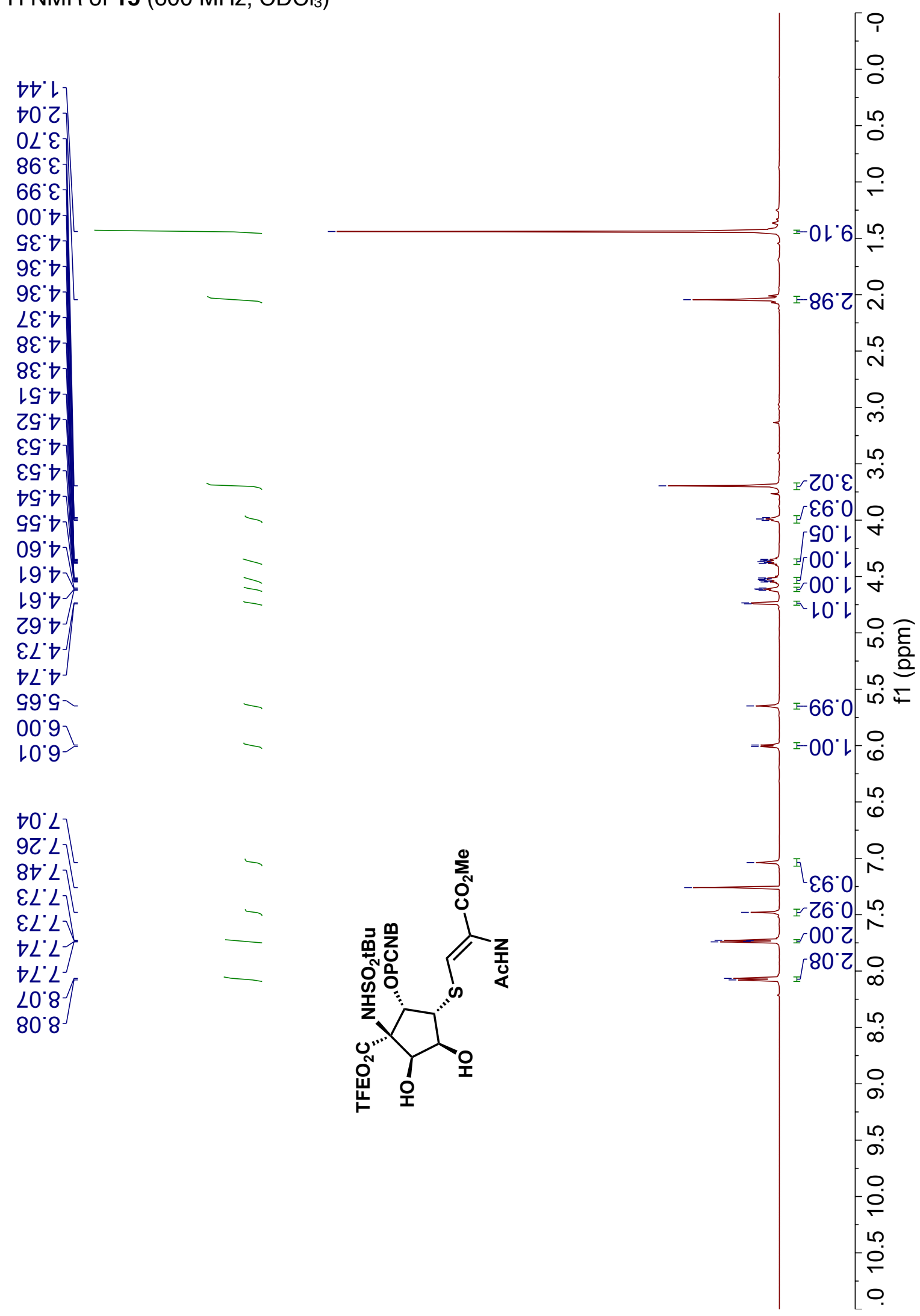
${ }^{13} \mathrm{C}$ NMR of $15\left(150 \mathrm{MHz}, \mathrm{CDCl}_{3}\right)$

$\downarrow Z^{\prime} \varepsilon Z$

$0 l^{\circ} \nabla Z^{\prime}$

$9\llcorner\cdot 29$

$\nabla Z * \nabla G$

06.09

$\varepsilon t^{\prime} \cdot 9$

$\angle 9.19$

$26 \cdot 19$

$9 l^{\circ} \mathrm{Z9}$

GS'lL】

$\angle V^{\circ} \nabla L C$

$95.92-$

91.21

$\angle \varepsilon^{\prime} L L$

ZZ: $\angle L L$

$16 \cdot 2 L 1$

68 ' L

EL'LL

9G'EZL

Ot:GZL

$\varepsilon 9.0 \varepsilon 1$ -

S†'ZعL

$8 \nabla^{\circ} Z \varepsilon L$

$\neg 8^{\circ} 9 \varepsilon \downarrow$

$6 \varepsilon^{\prime} \varepsilon 9 L$

ง6. $891-$

ZL'L9LT

Z9. $89 \mathrm{l}$

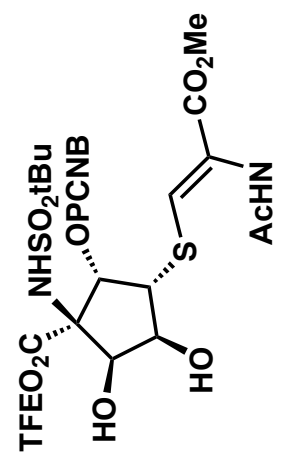

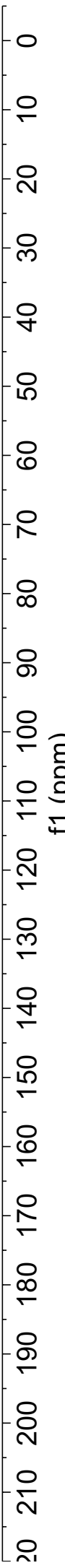


${ }^{19} \mathrm{~F} \mathrm{NMR} \mathrm{of} 15\left(376 \mathrm{MHz}, \mathrm{CDCl}_{3}\right)$

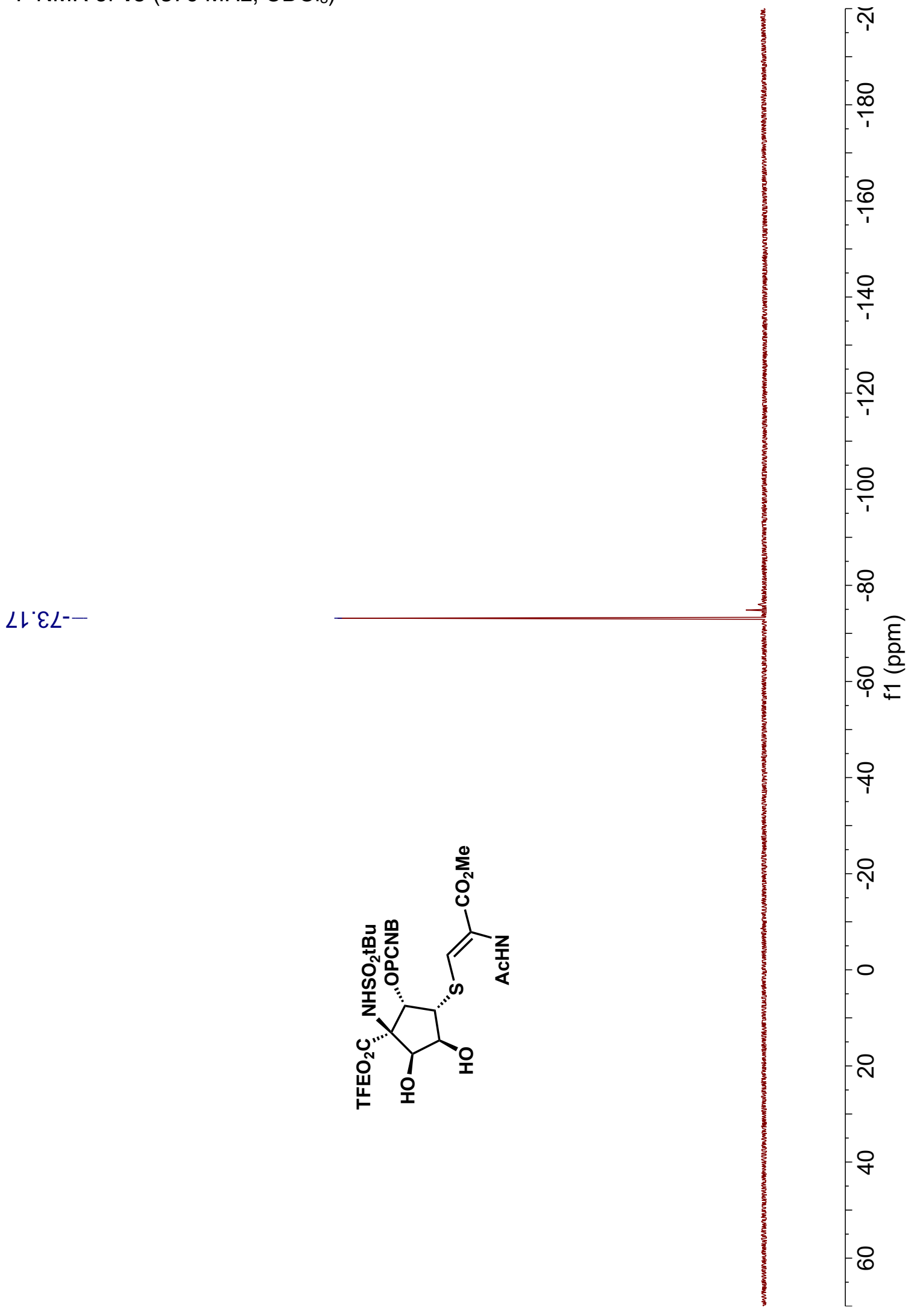


HSQC of 15

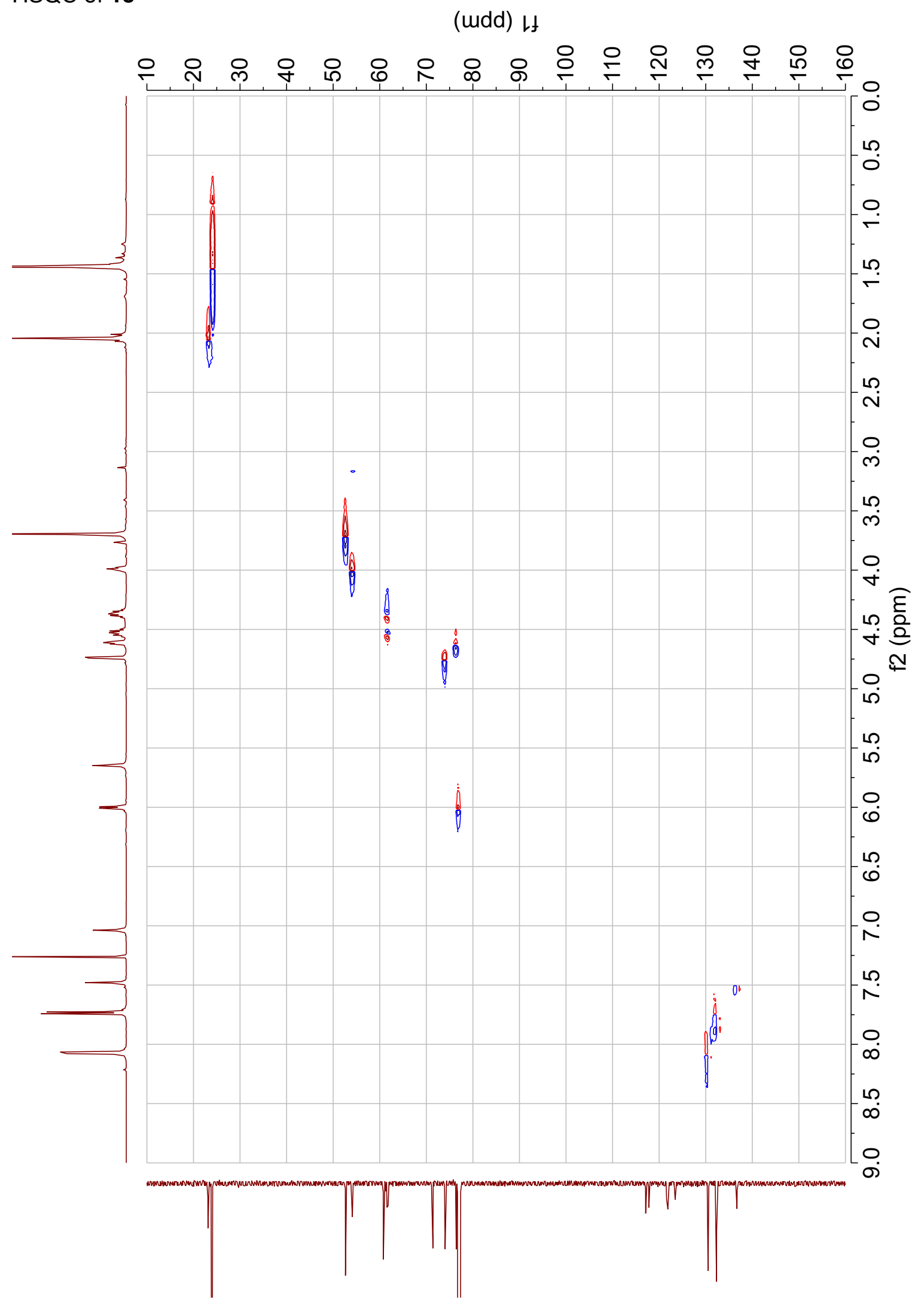


(udd) If

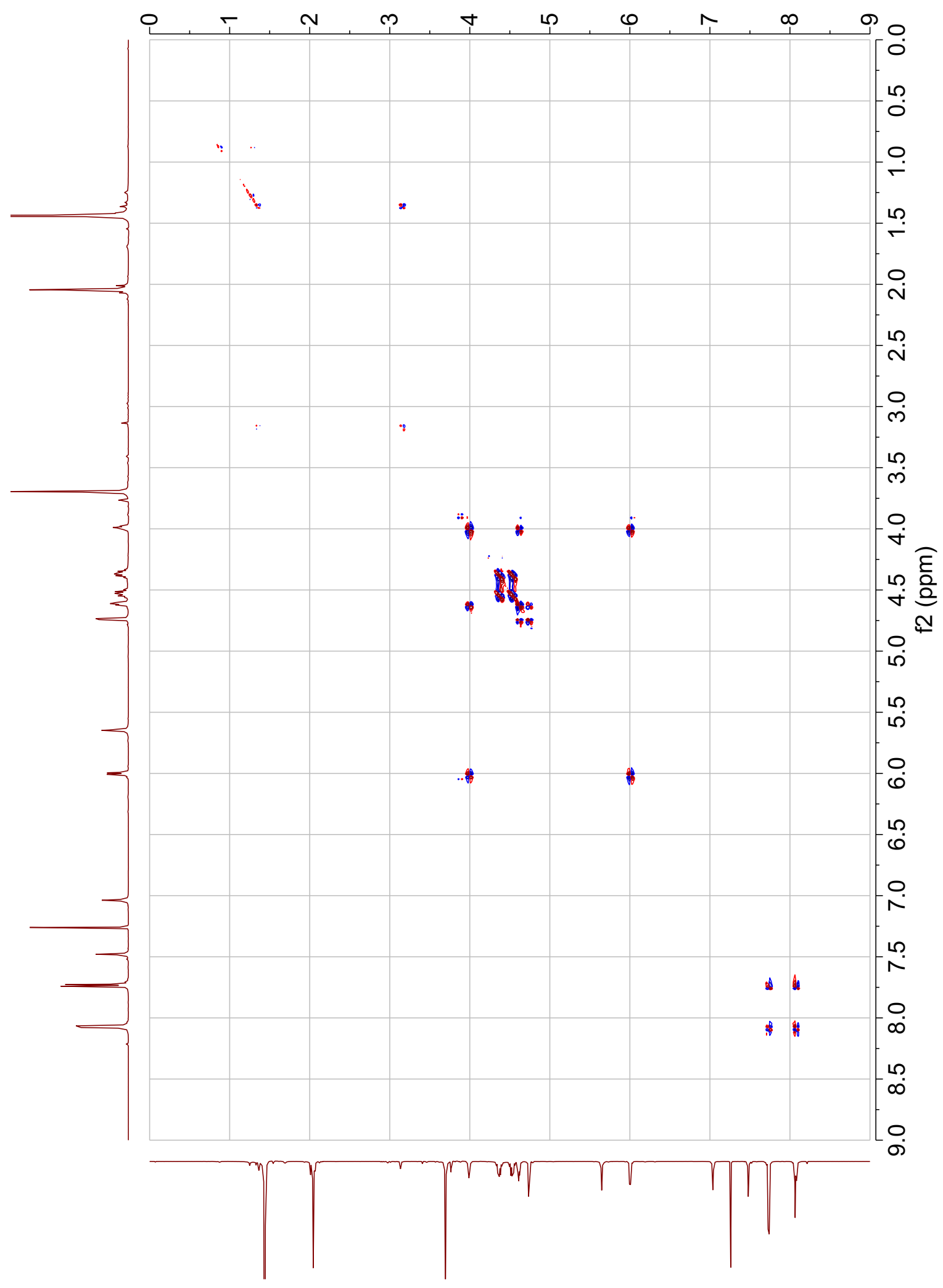


${ }^{1} \mathrm{H}$ NMR of $16\left(600 \mathrm{MHz}, \mathrm{CDCl}_{3}\right)$

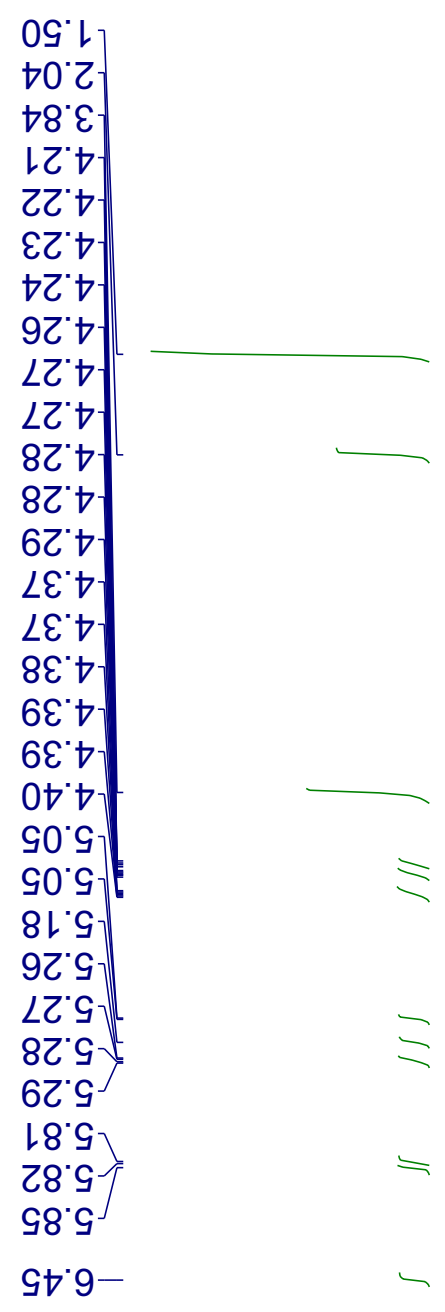

$9 Z^{\circ} L-$

$G L L]$

86.1

00.8

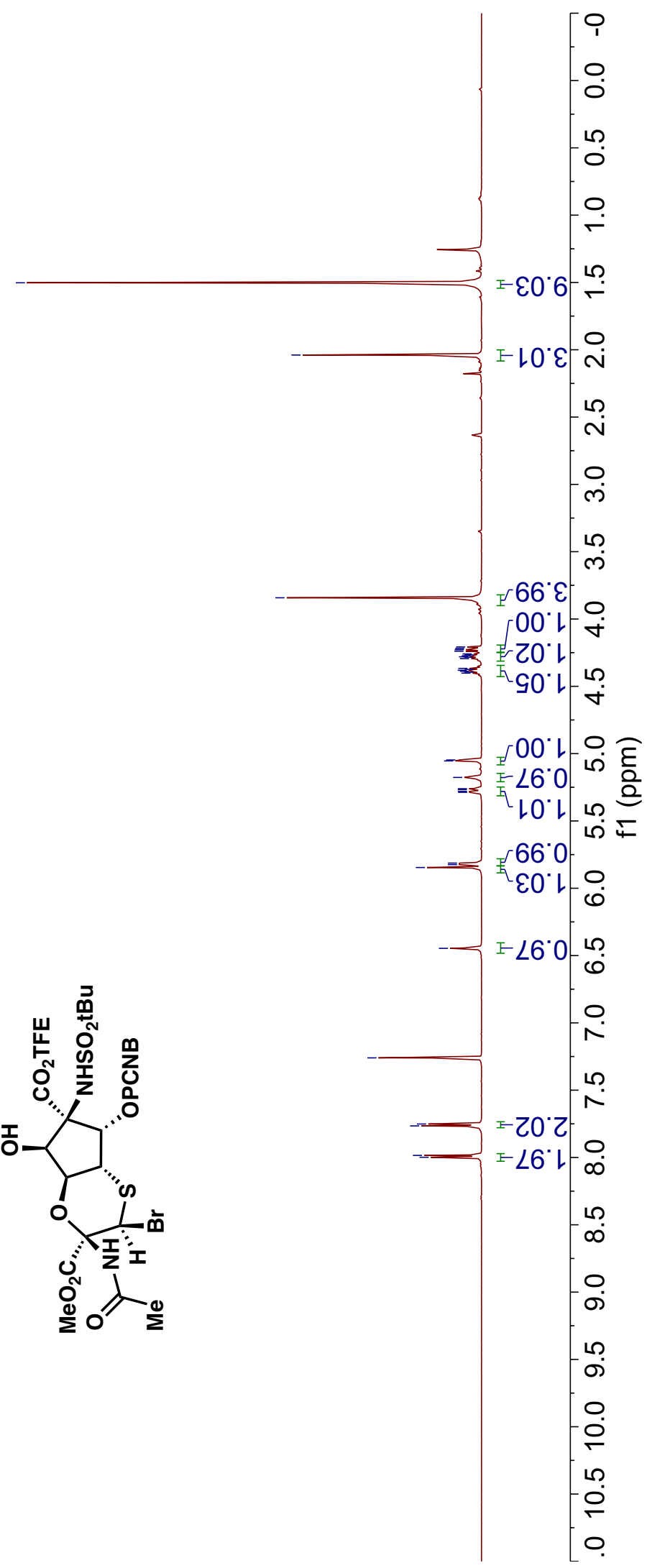


${ }^{13} \mathrm{C}$ NMR of $16\left(150 \mathrm{MHz}, \mathrm{CDCl}_{3}\right)$
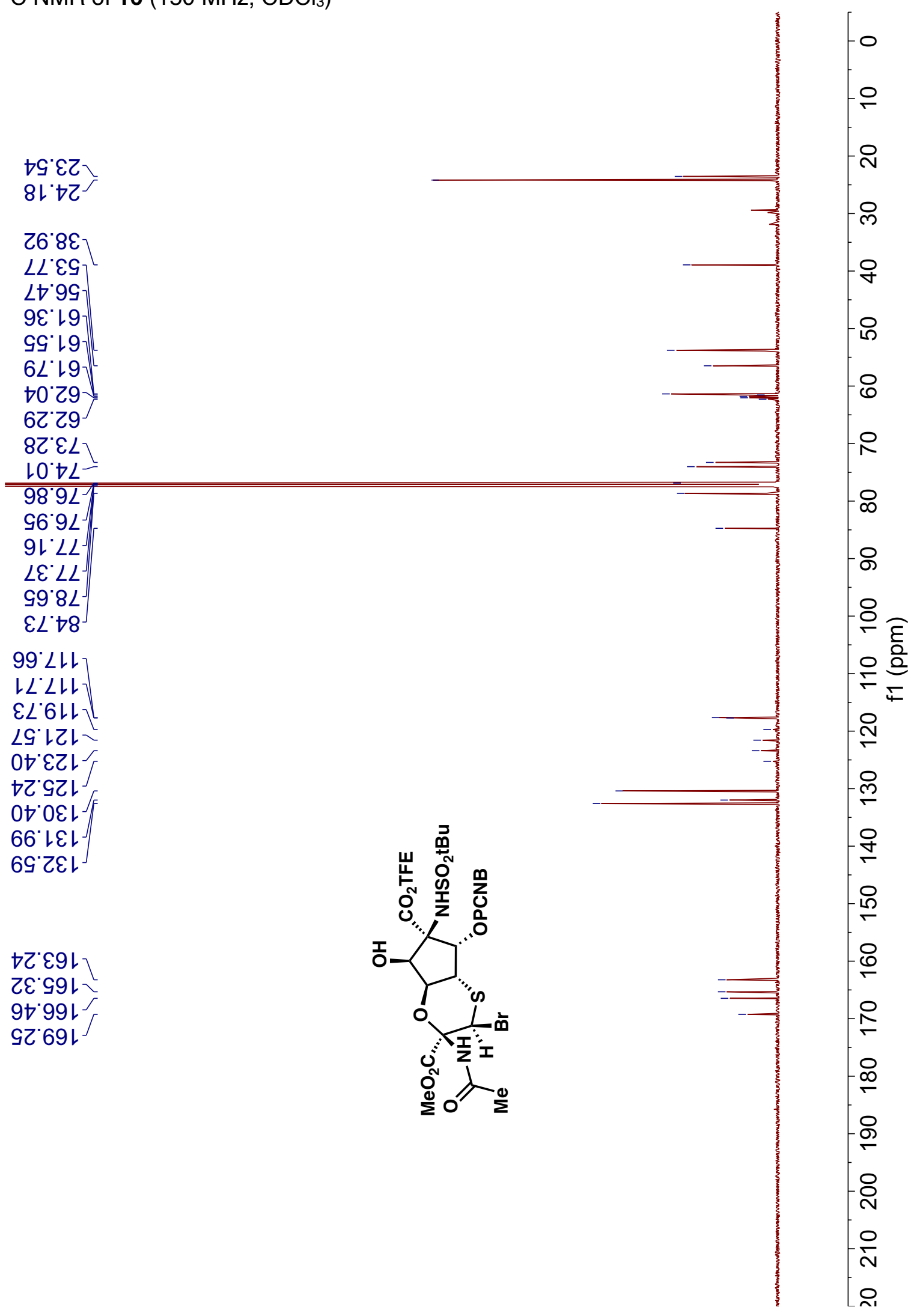
${ }^{19} \mathrm{~F} \mathrm{NMR}$ of $16\left(376 \mathrm{MHz}, \mathrm{CDCl}_{3}\right)$

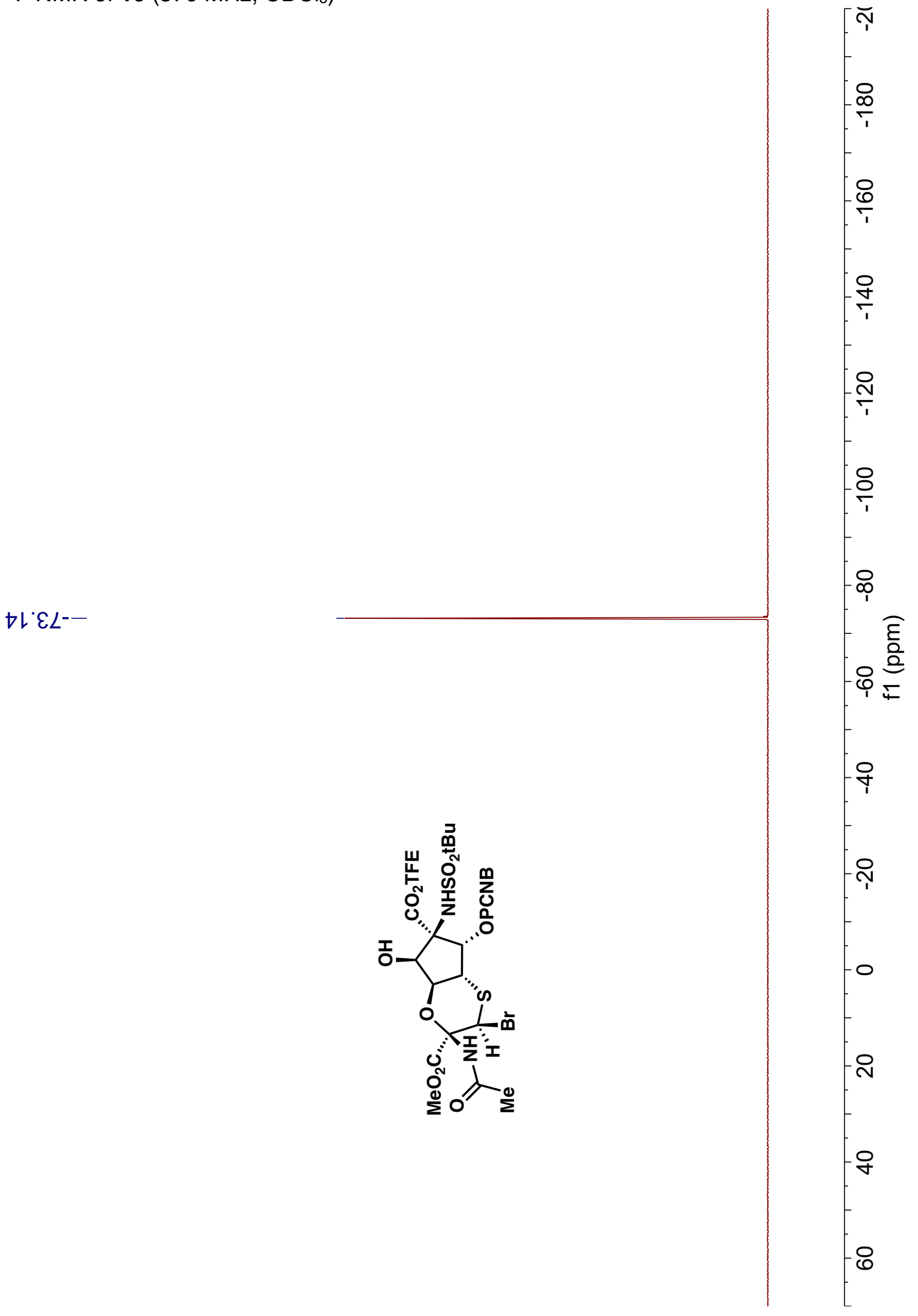


${ }^{1} \mathrm{H}$ NMR of $17\left(600 \mathrm{MHz}, \mathrm{CDCl}_{3}\right)$

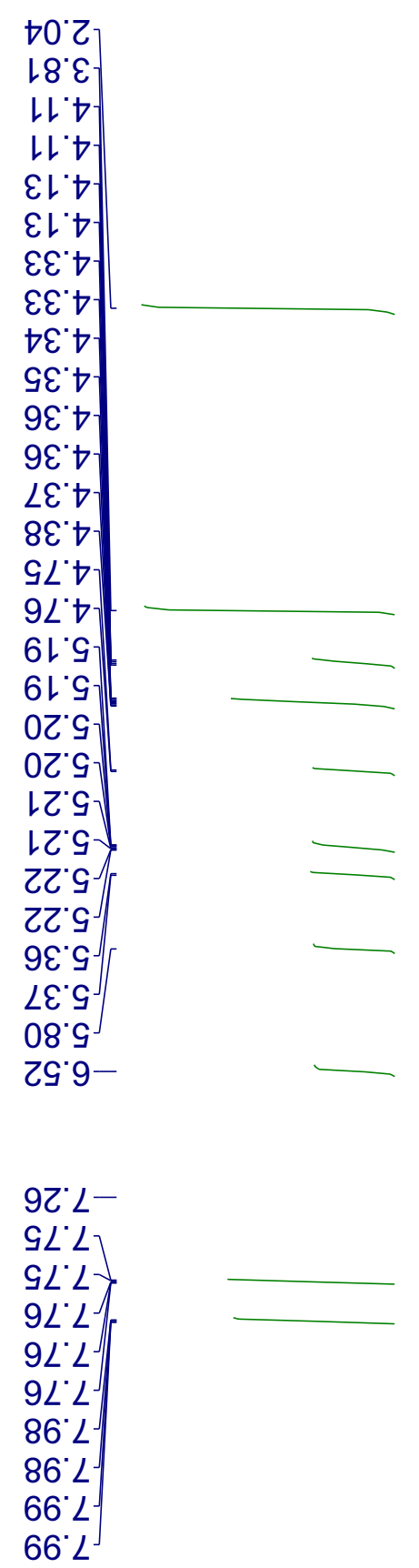

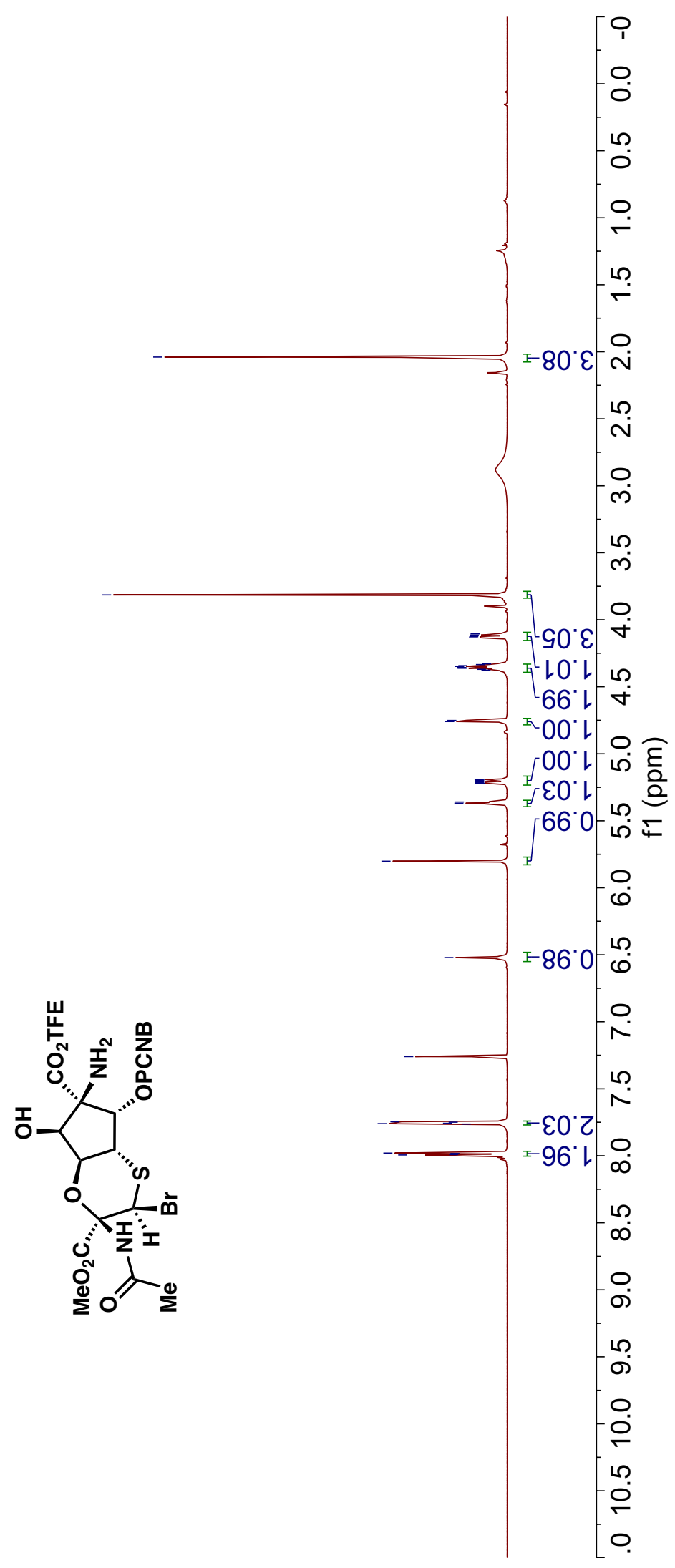


${ }^{13} \mathrm{C}$ NMR of $17\left(150 \mathrm{MHz}, \mathrm{CDCl}_{3}\right)$

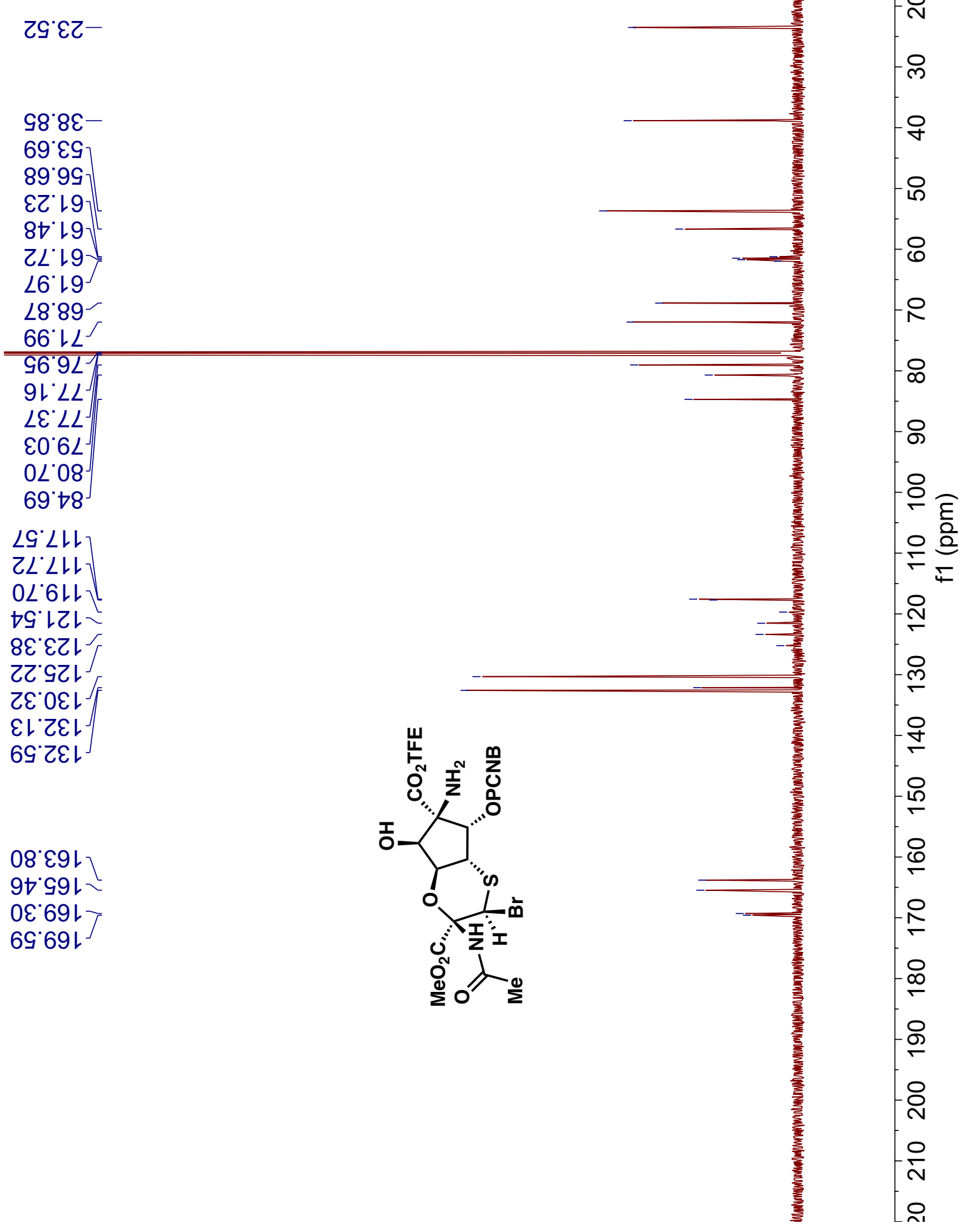


${ }^{19} \mathrm{~F} \mathrm{NMR}$ of $17\left(376 \mathrm{MHz}, \mathrm{CDCl}_{3}\right)$

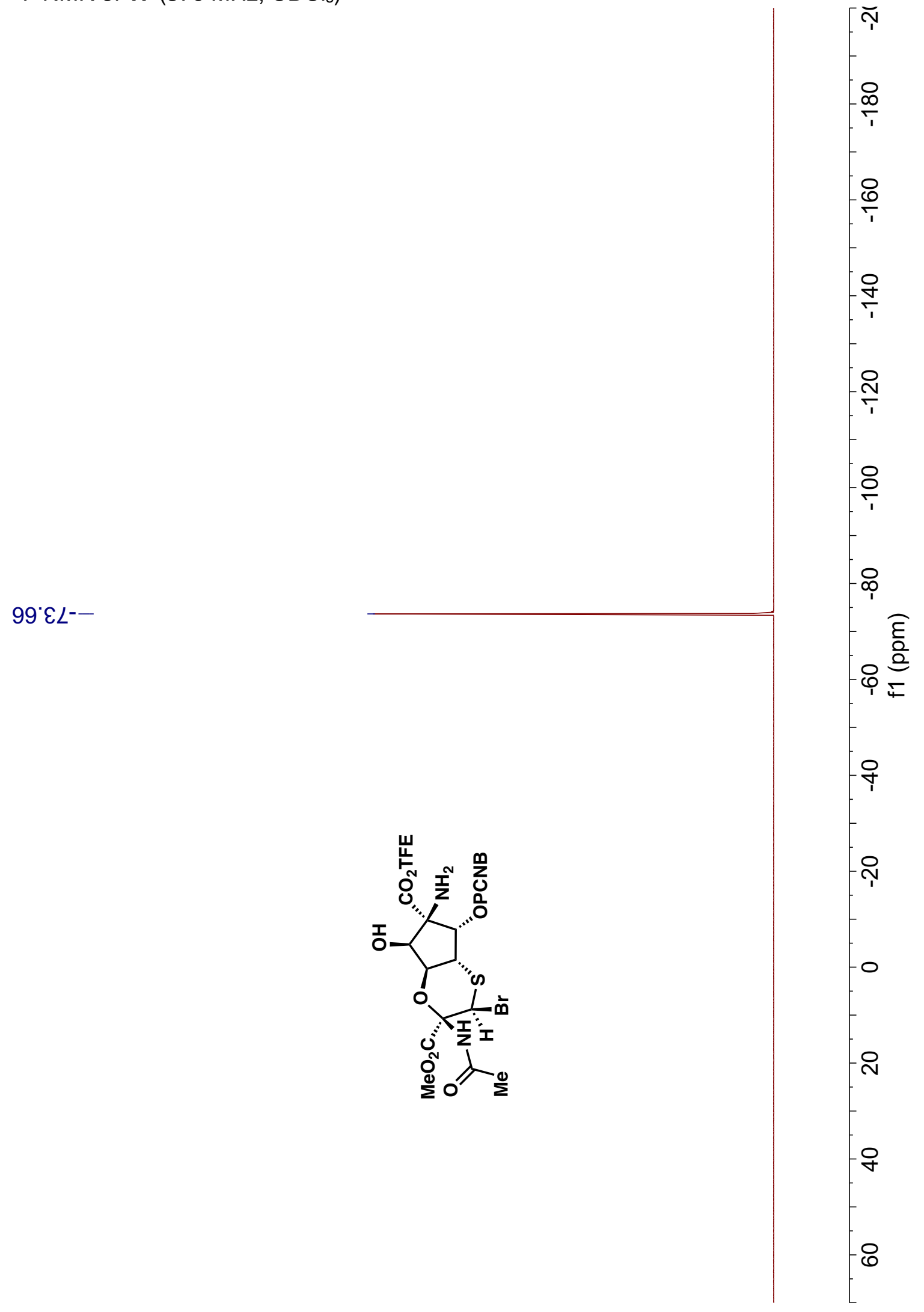


${ }^{1} \mathrm{H}$ NMR of $18\left(600 \mathrm{MHz}\right.$, acetone- $\left.d_{6}\right)$

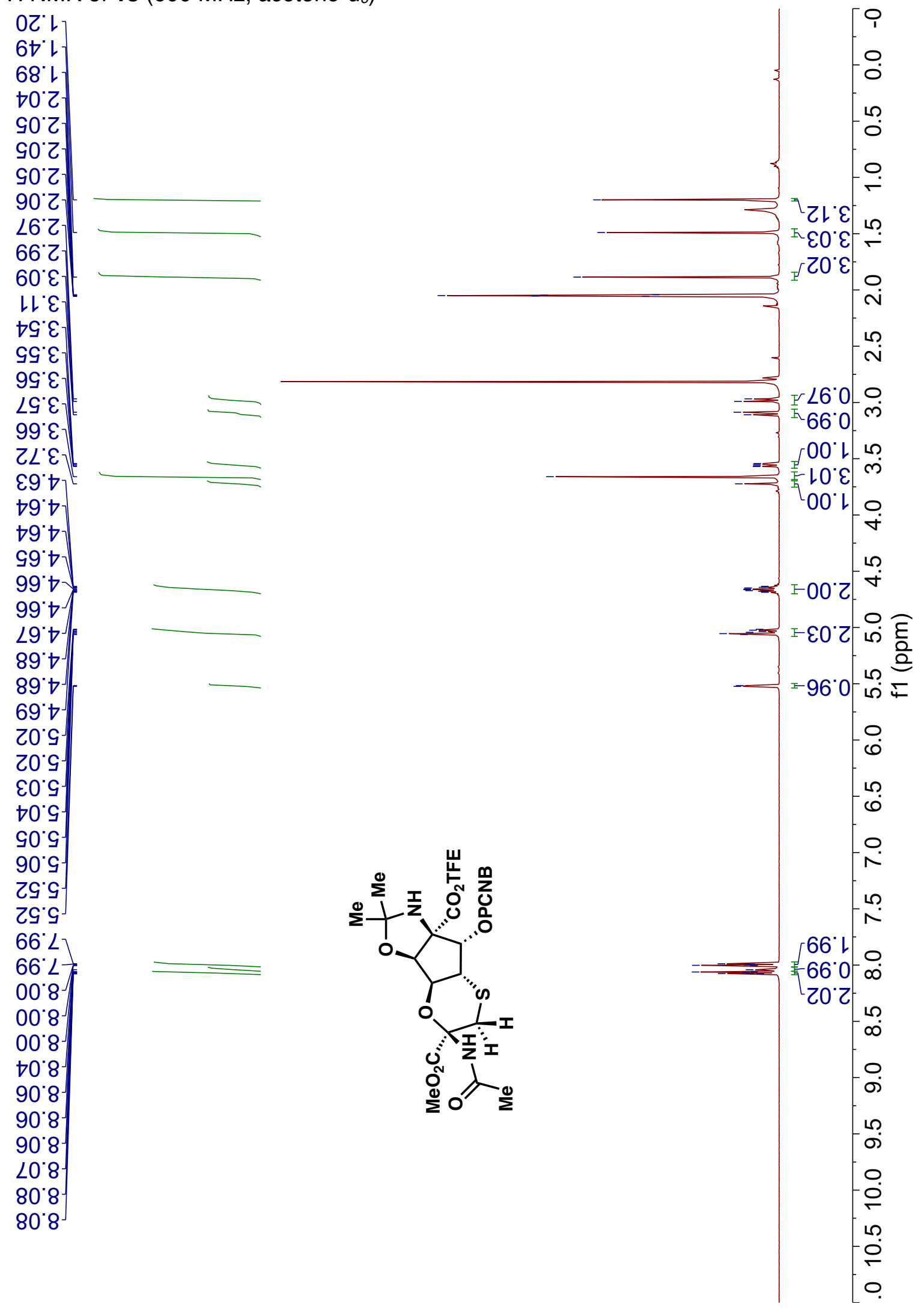




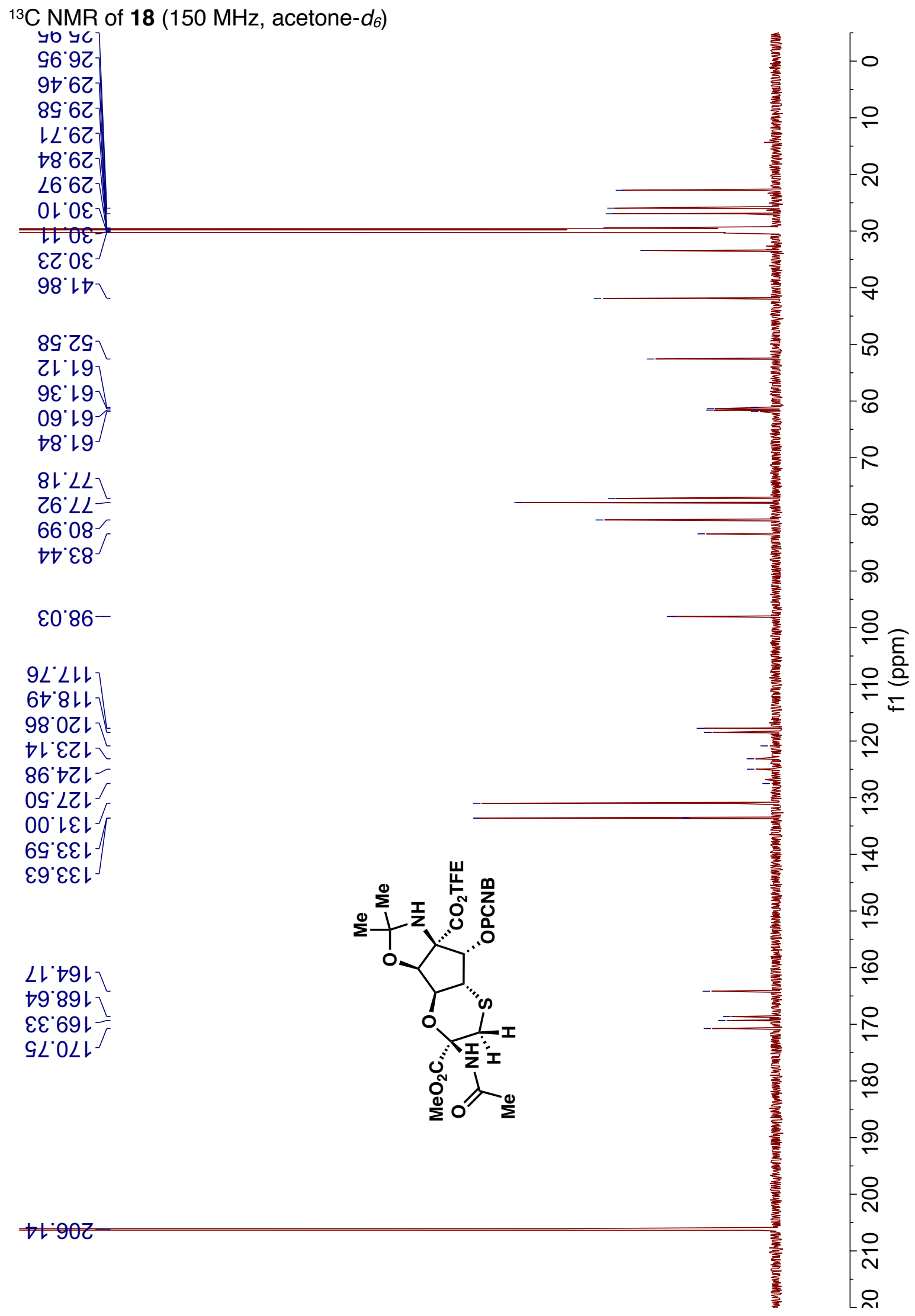


${ }^{19} \mathrm{~F}$ NMR of $18\left(376 \mathrm{MHz}\right.$, acetone- $\left.d_{6}\right)$

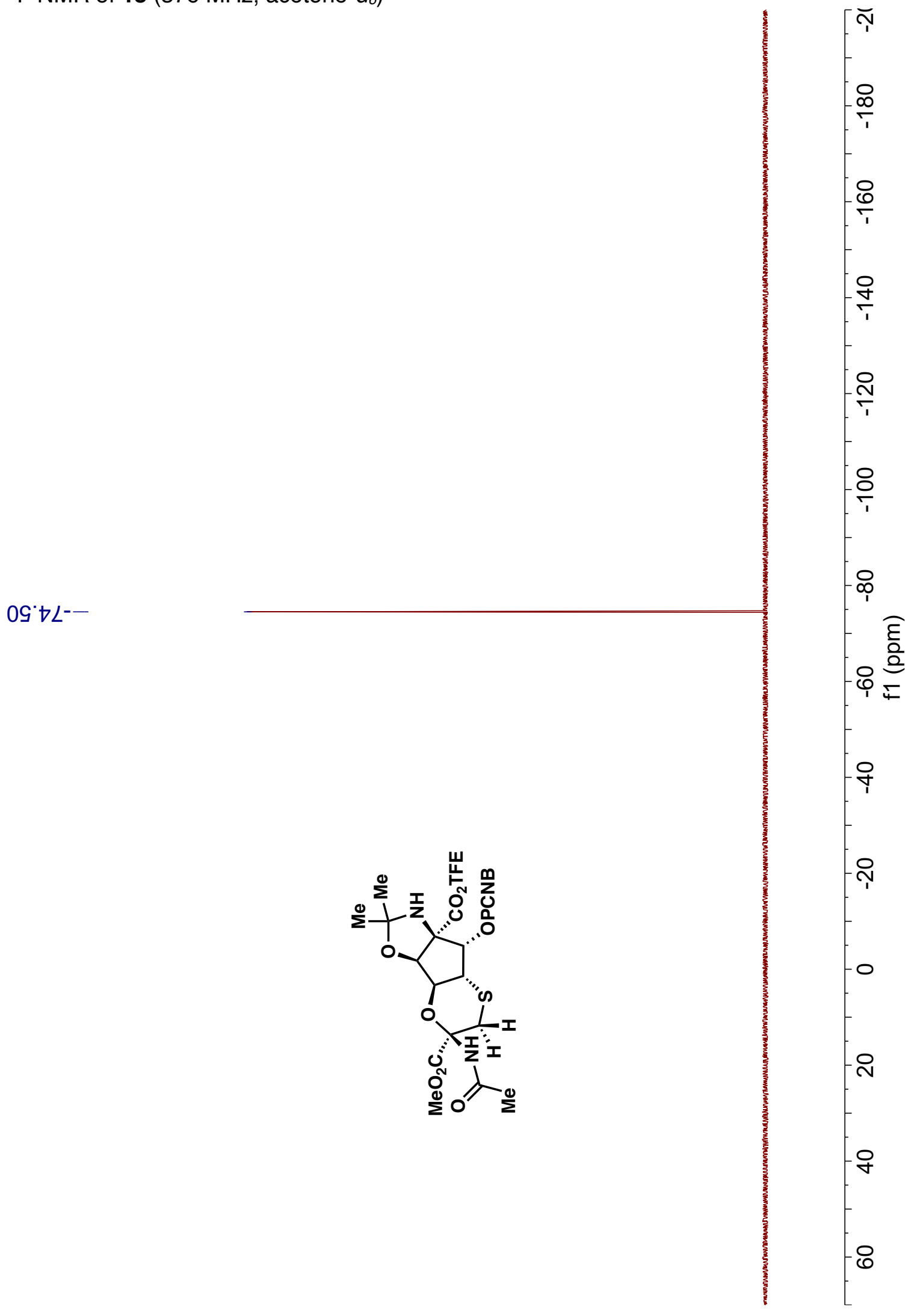


${ }^{1} \mathrm{H}$ NMR of $19 \mathrm{a}\left(600 \mathrm{MHz}, \mathrm{CD}_{3} \mathrm{CN}\right)$

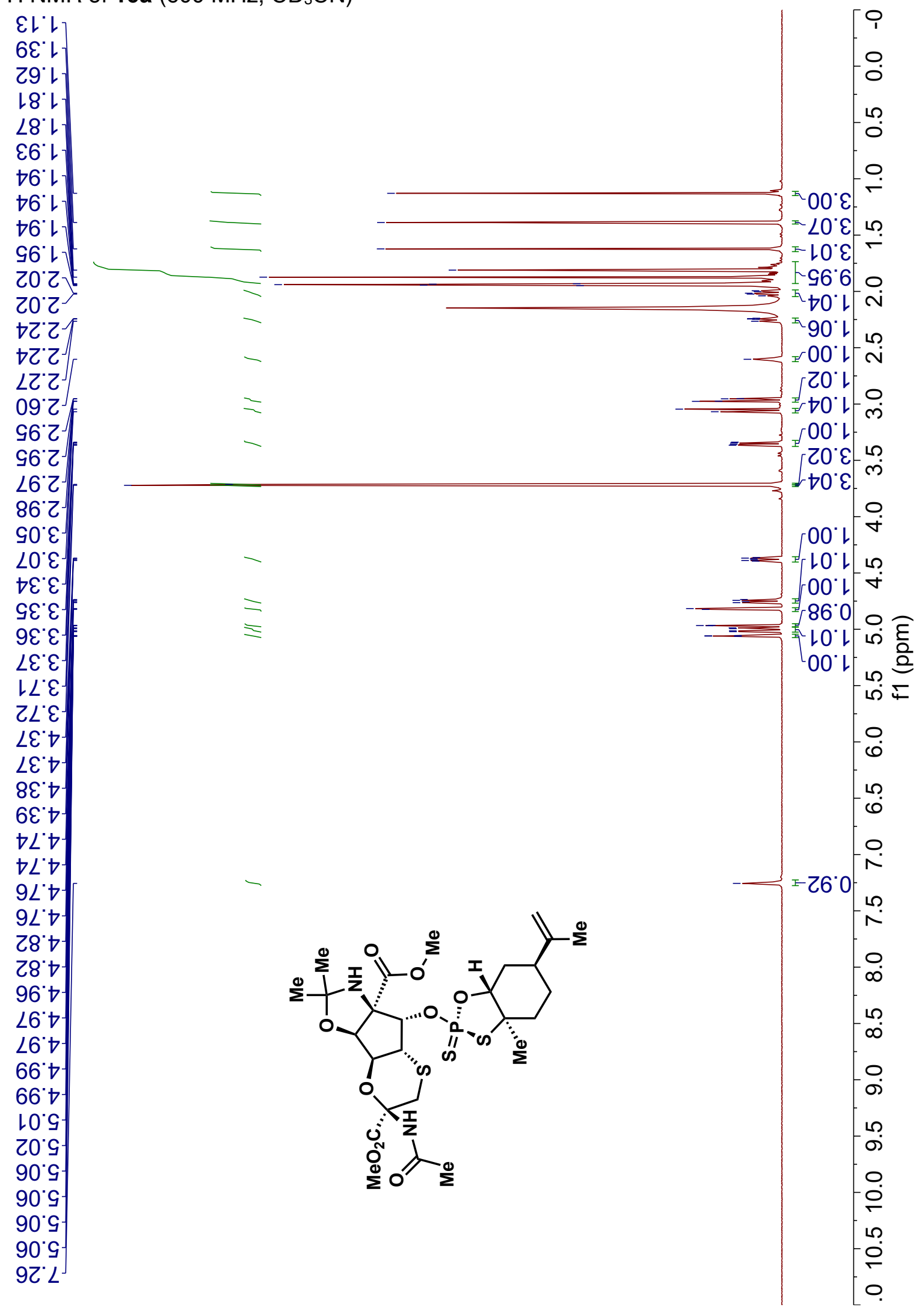




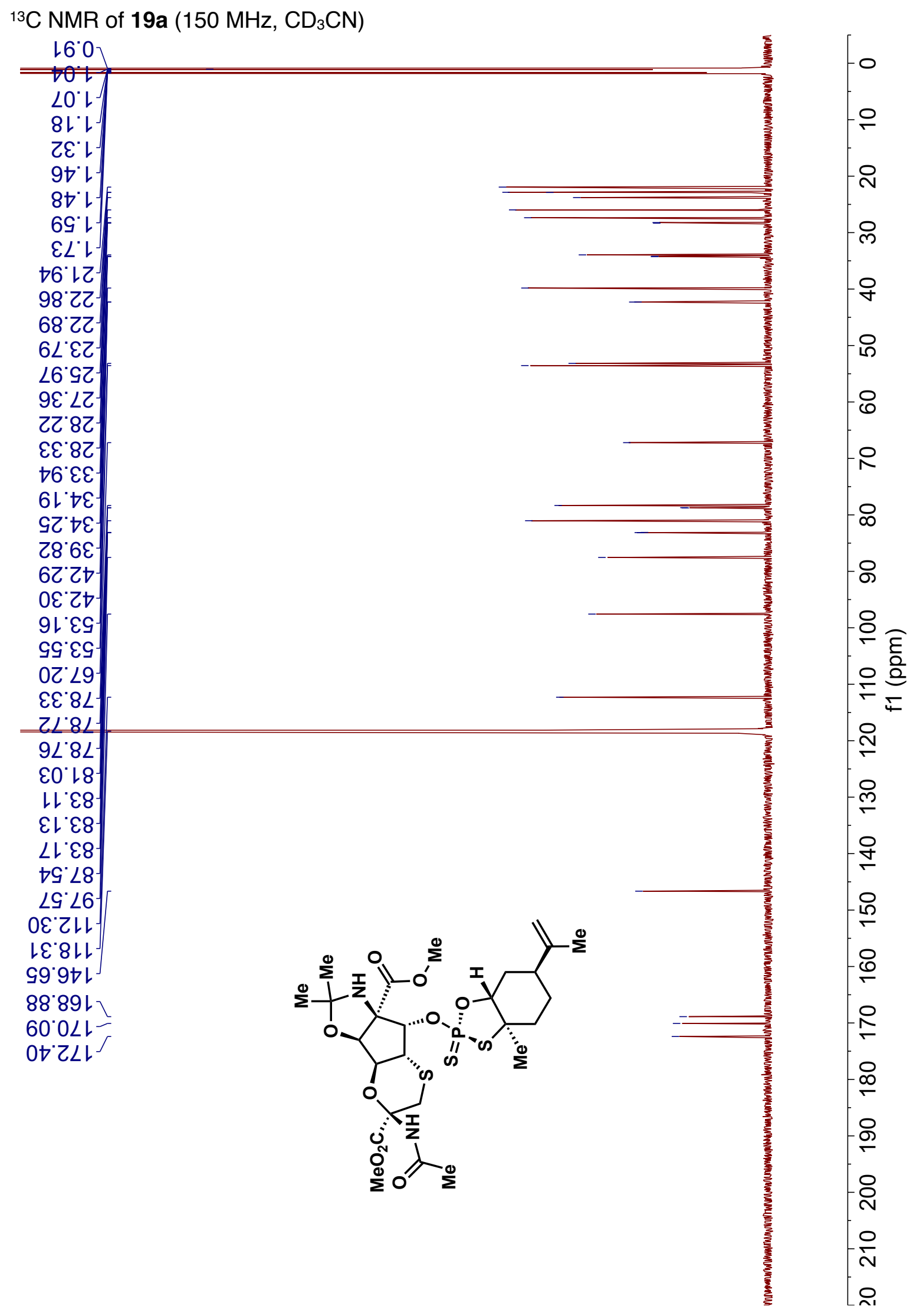


${ }^{31} \mathrm{P}$ NMR of $19 \mathrm{a}\left(162 \mathrm{MHz}, \mathrm{CD}_{3} \mathrm{CN}\right)$

00รัเ-

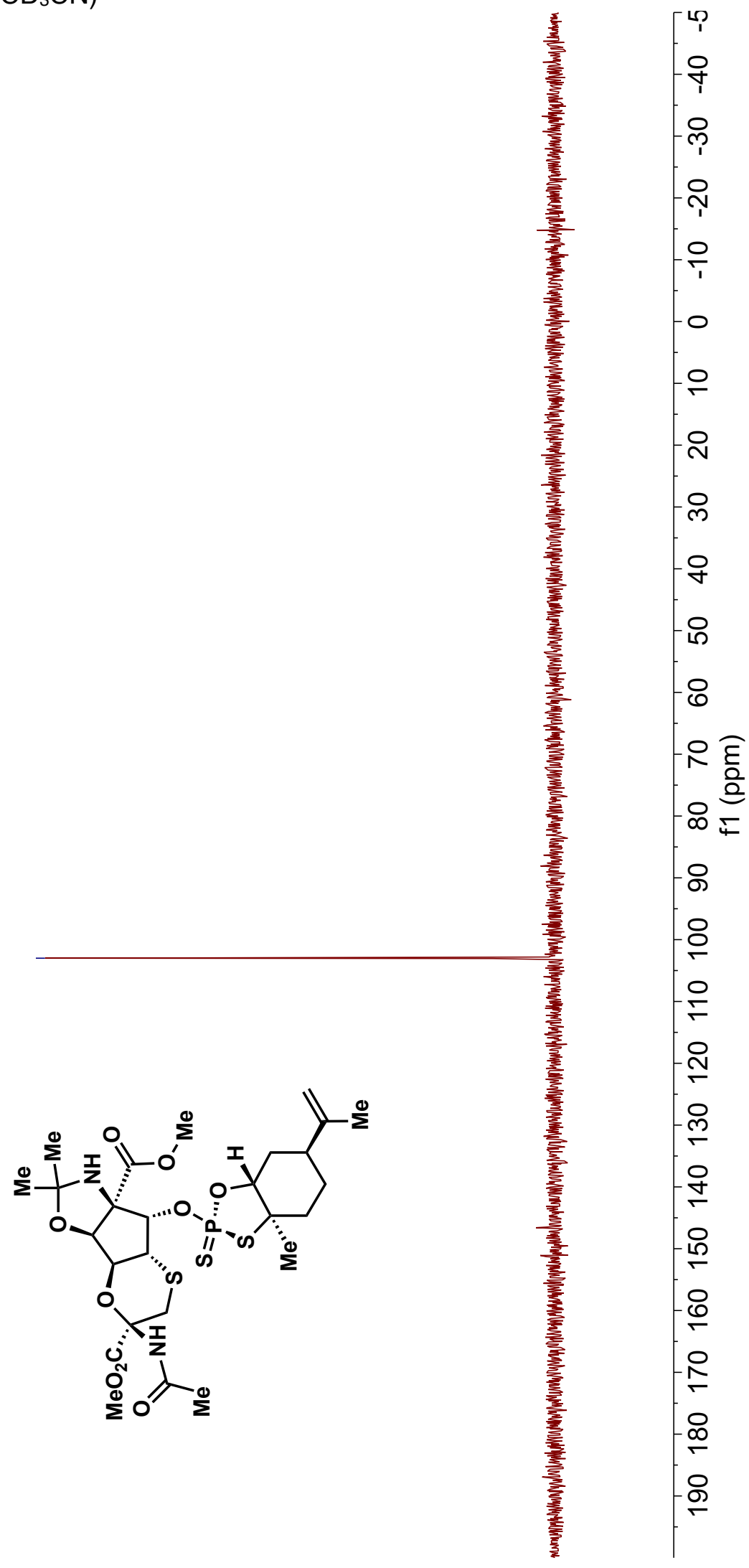




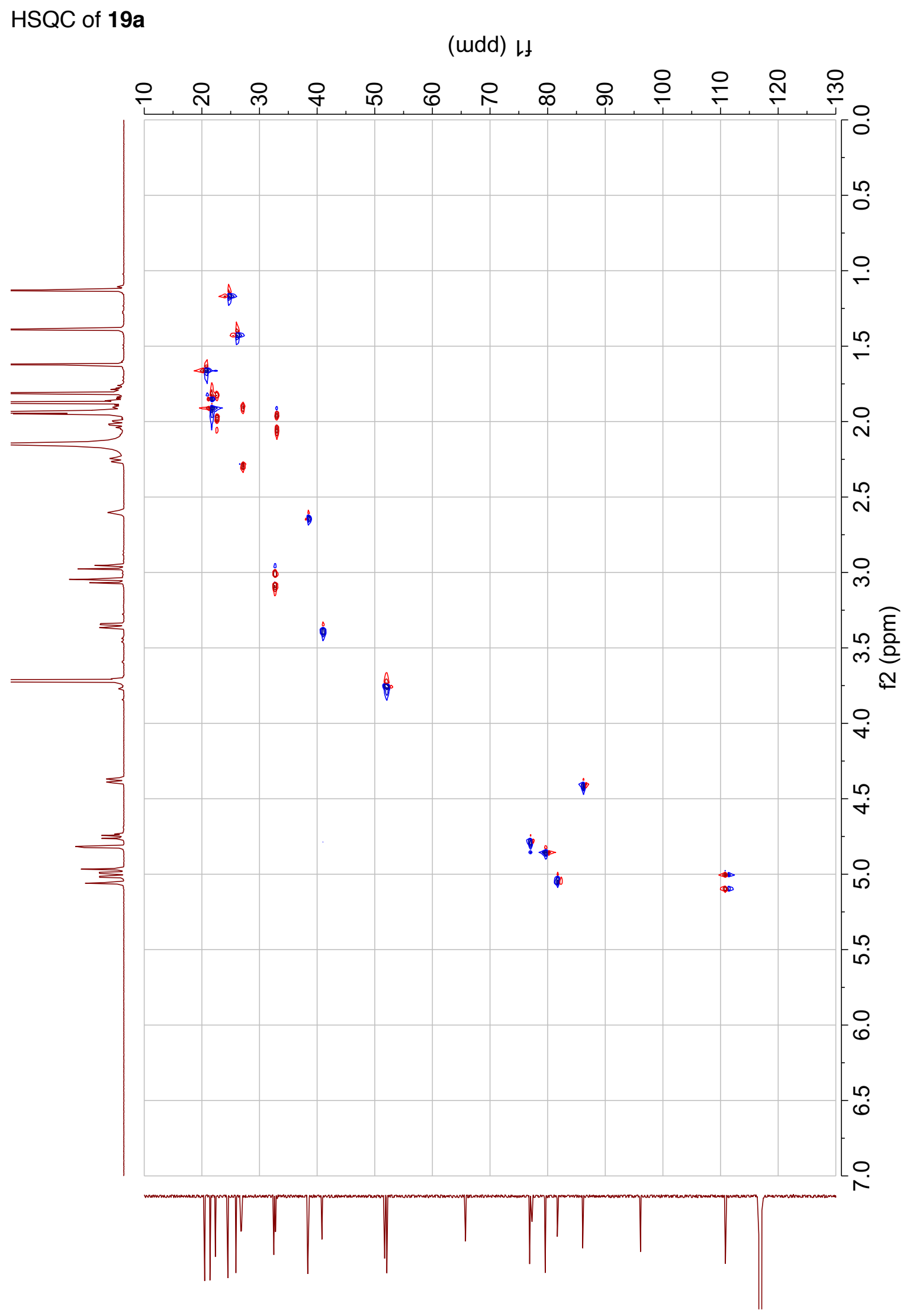




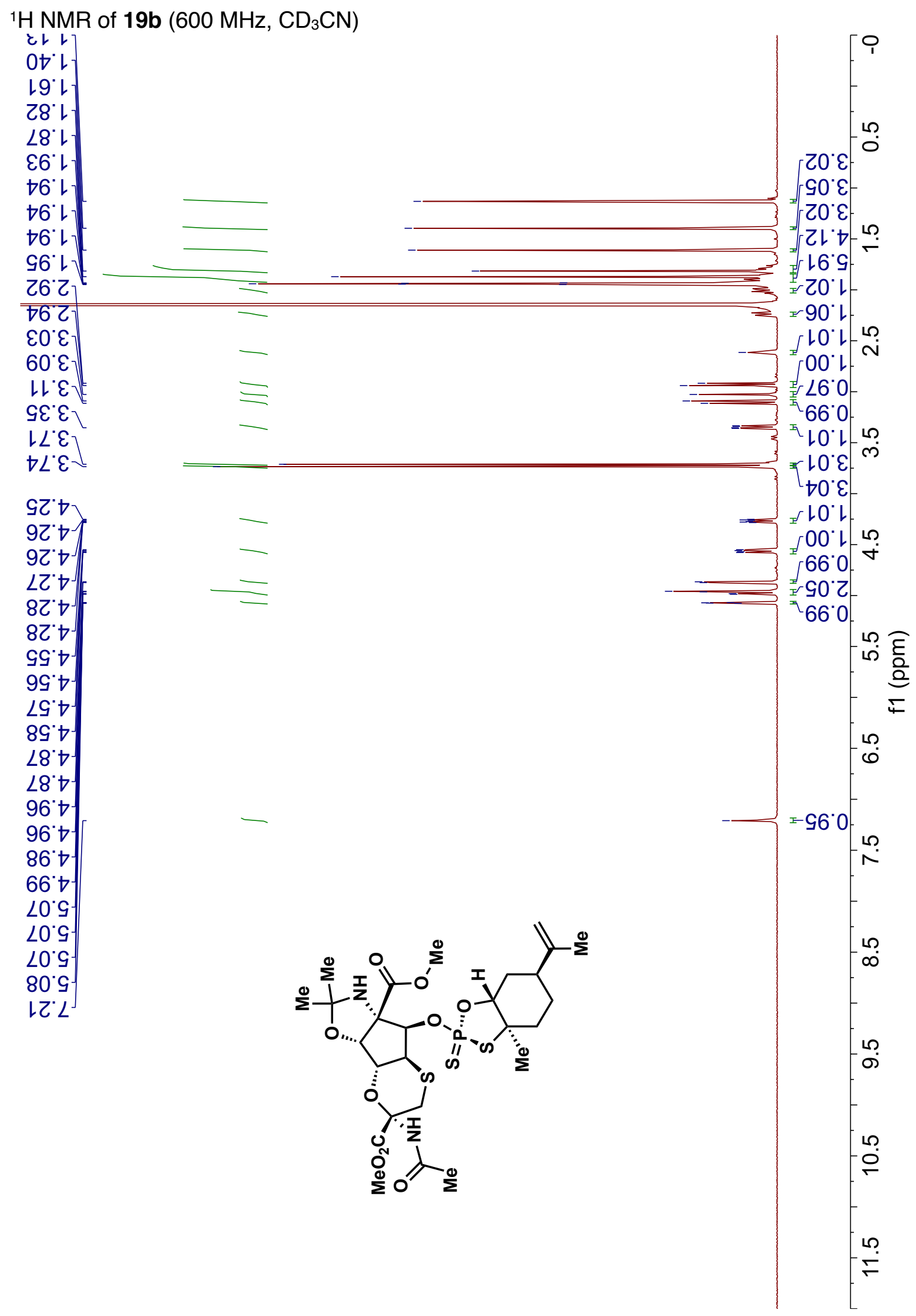


${ }^{13} \mathrm{C}$ NMR of $19 b\left(150 \mathrm{MHz}, \mathrm{CD}_{3} \mathrm{CN}\right)$

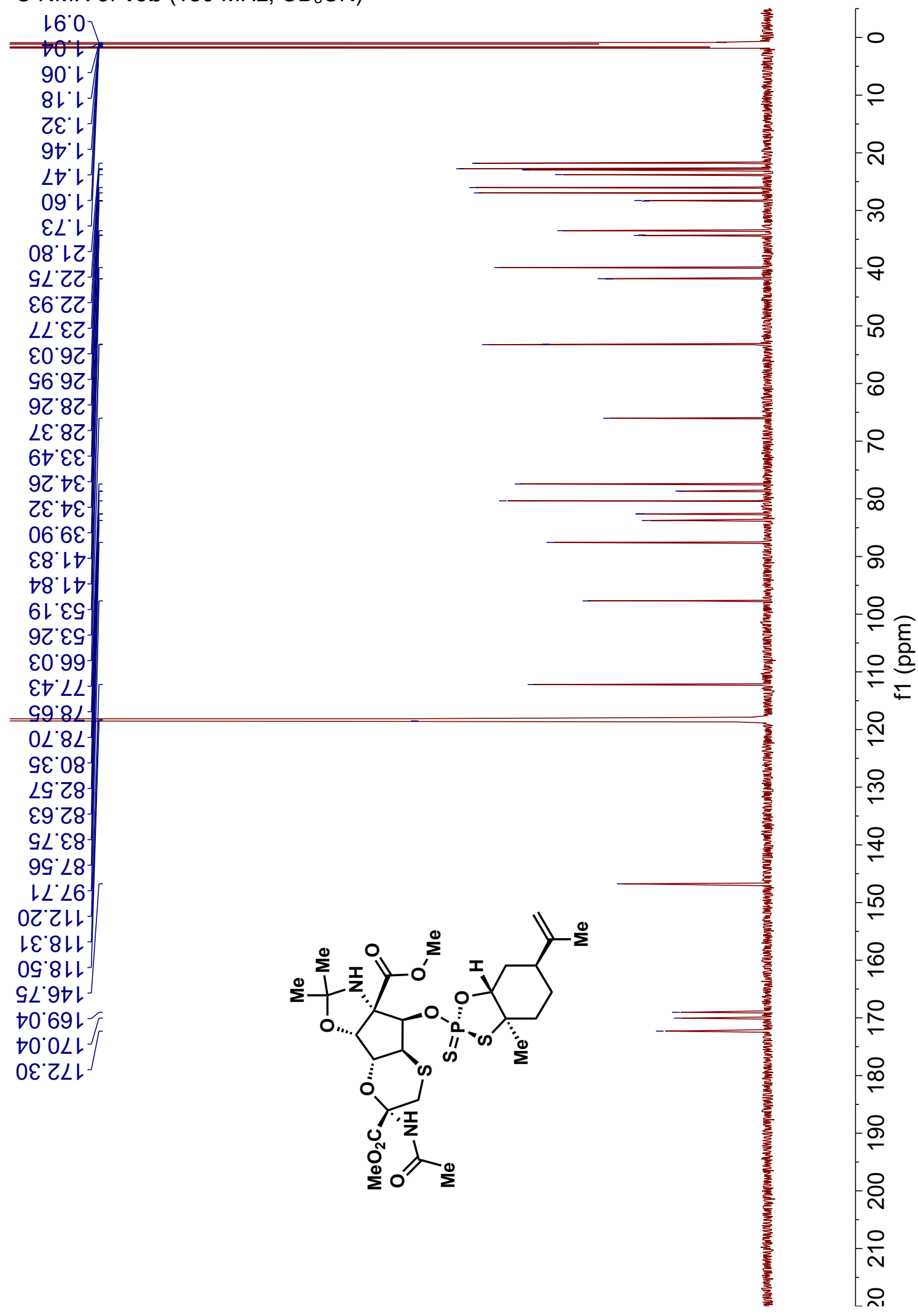


${ }^{31} \mathrm{P}$ NMR of $19 \mathbf{b}\left(162 \mathrm{MHz}, \mathrm{CD}_{3} \mathrm{CN}\right)$

$\succ 0^{\circ} \varepsilon 0\llcorner-$

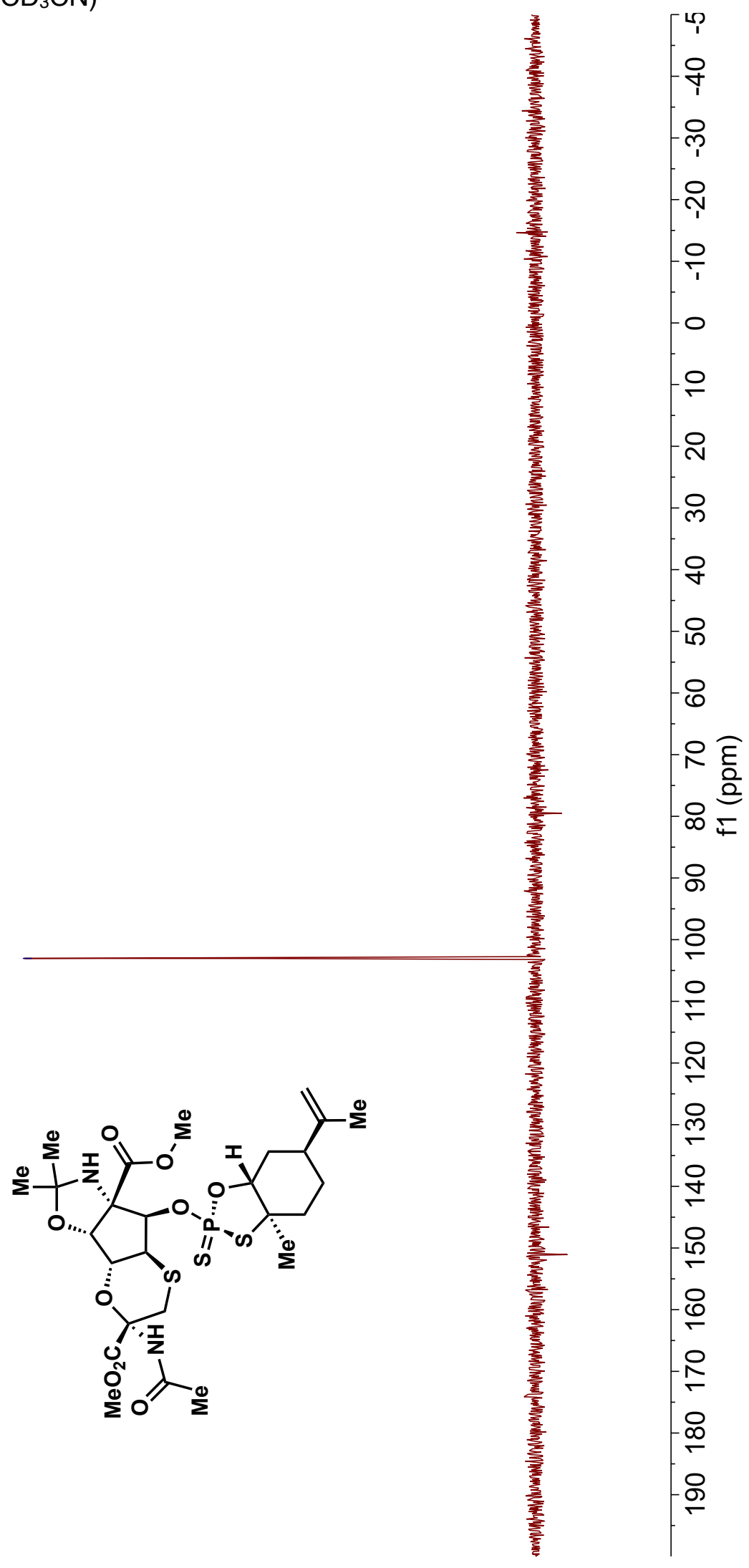




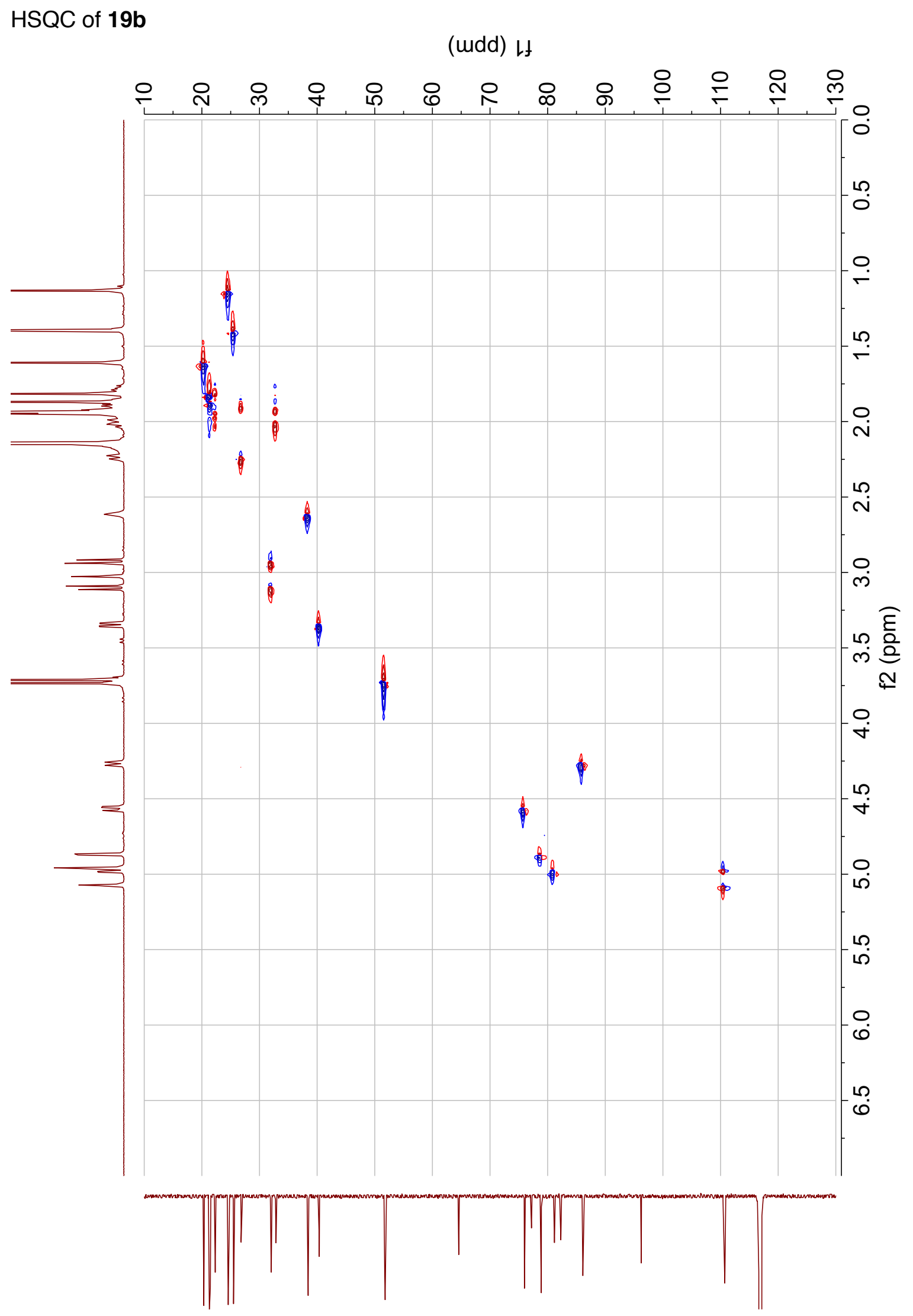


${ }^{1} \mathrm{H}$ NMR of $(+)-1\left(600 \mathrm{MHz}, 0.05 \mathrm{M} \mathrm{NH}_{4} \mathrm{HCO}_{3}\right.$ in $\left.\mathrm{D}_{2} \mathrm{O}\right)$

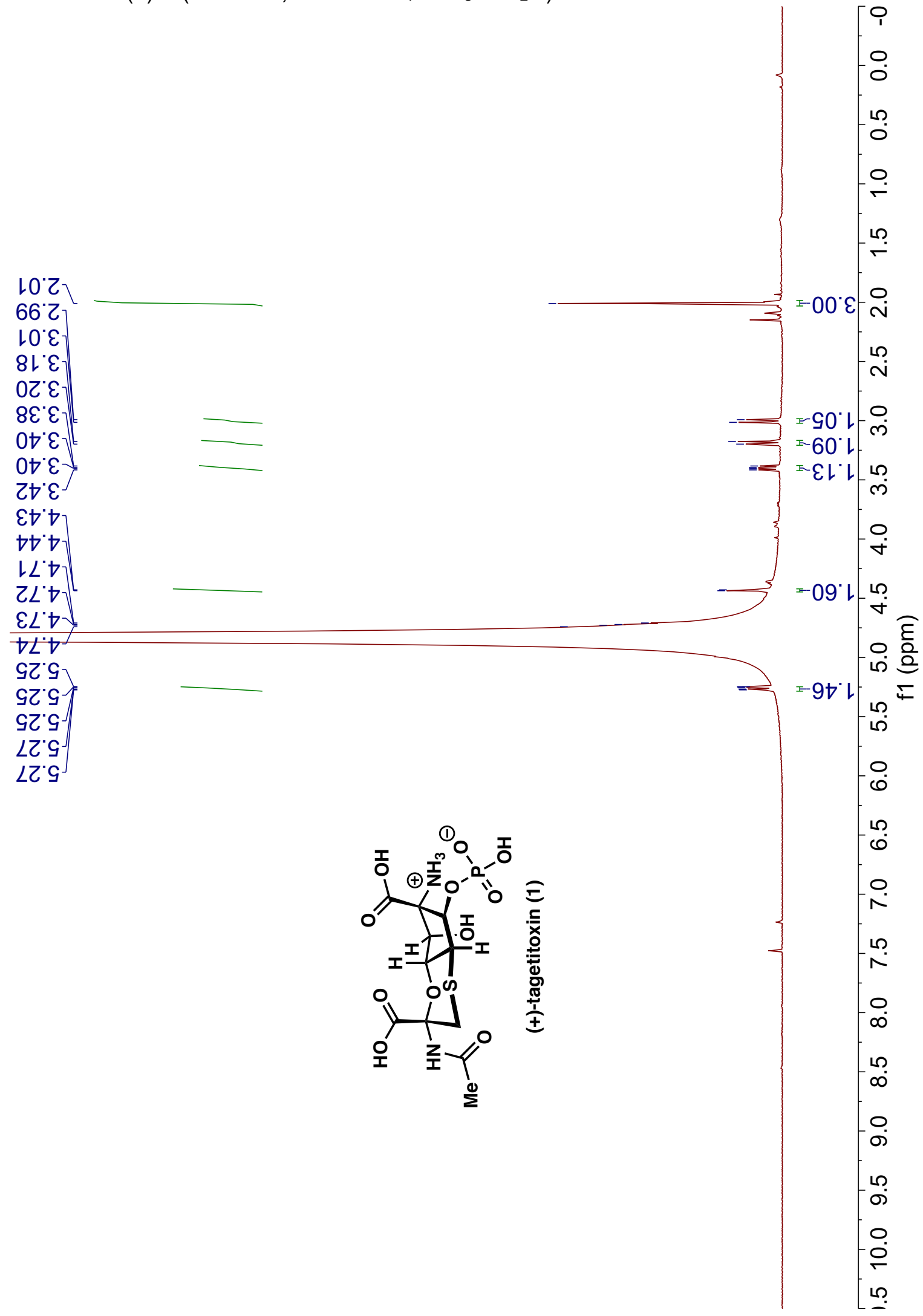


${ }^{1} \mathrm{H}$ NMR of $(+)-1\left(600 \mathrm{MHz}, 0.05 \mathrm{M} \mathrm{NH}_{4} \mathrm{HCO}_{3}\right.$ in $\mathrm{D}_{2} \mathrm{O}$, zoom in)

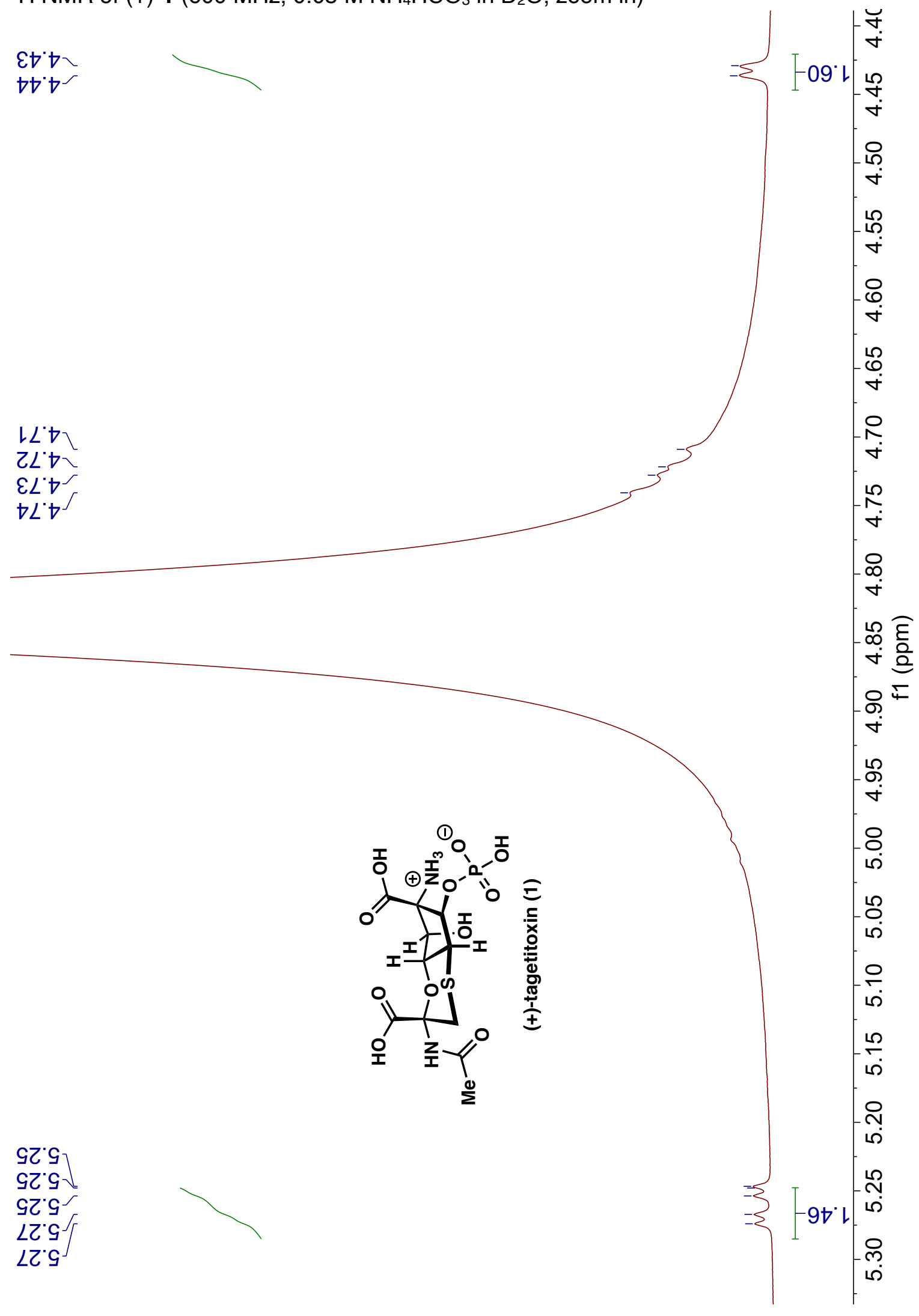


${ }^{13} \mathrm{C}$ NMR of $(+)-1\left(150 \mathrm{MHz}, 0.05 \mathrm{M} \mathrm{NH}_{4} \mathrm{HCO}_{3}\right.$ in $\left.\mathrm{D}_{2} \mathrm{O}\right)$

$18^{\circ} 2 Z^{-}$

$\varepsilon 9^{\circ} \varepsilon \varepsilon-$

$6 G^{\prime} \varepsilon b$

เ9. $\varepsilon \succ^{\top}$

จトเL

$\angle L \cdot L L V$

Z० $\varepsilon L-$

$\angle Z^{\prime} 9 L-1$

$0 \varepsilon^{\circ} 9 L$

06.62

$9 Z^{\circ} \mathrm{s}$

ZG'LLL

$89^{\circ} \varepsilon \angle L \div$
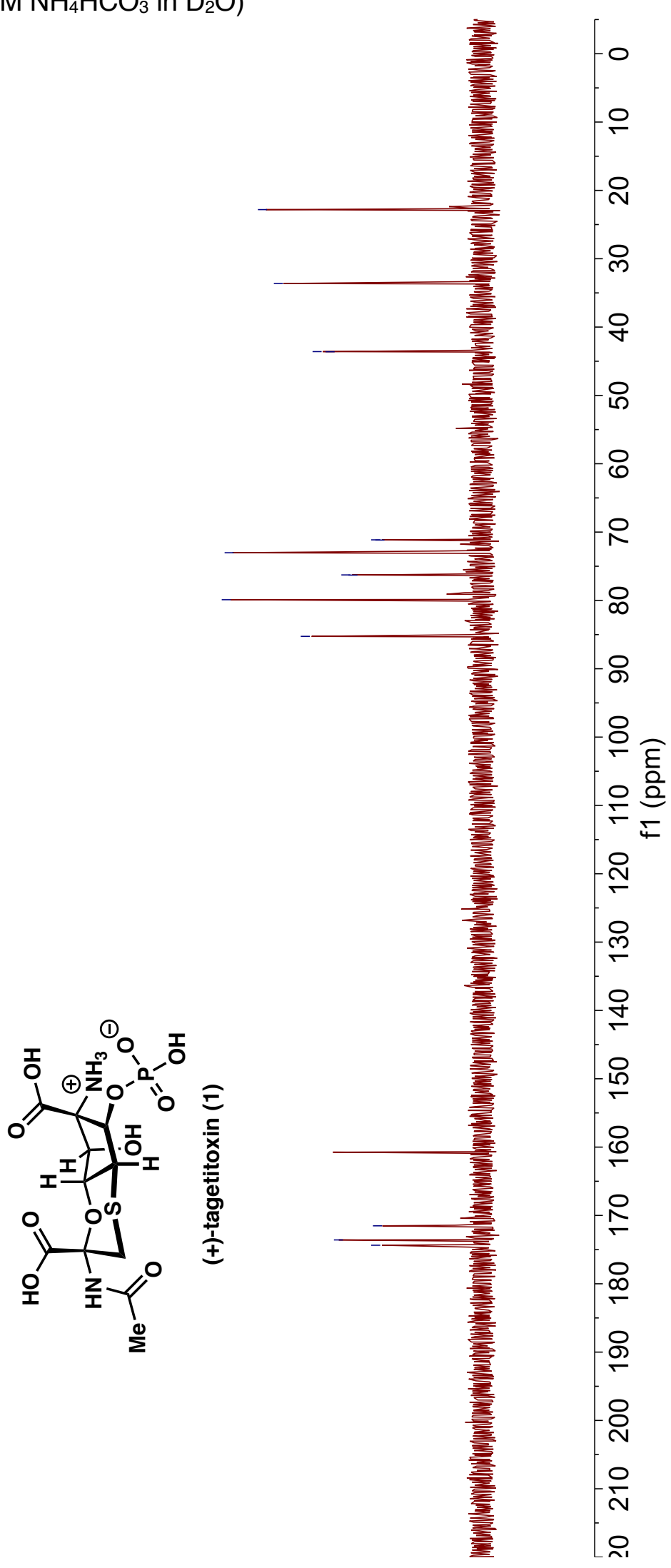

$9 \varepsilon^{\circ} \triangleright L \downarrow$ 
${ }^{13} \mathrm{C} \mathrm{NMR} \mathrm{of}(+)-1$ (150 MHz, $0.05 \mathrm{M} \mathrm{NH}_{4} \mathrm{HCO}_{3}$ in $\mathrm{D}_{2} \mathrm{O}$, zoom in)

tL.LLC

$\angle L L^{J}$

Z๐॰ $\varepsilon\llcorner-$

$\angle Z \cdot 9 L$

$0 \varepsilon^{\circ} 9 L^{J}$

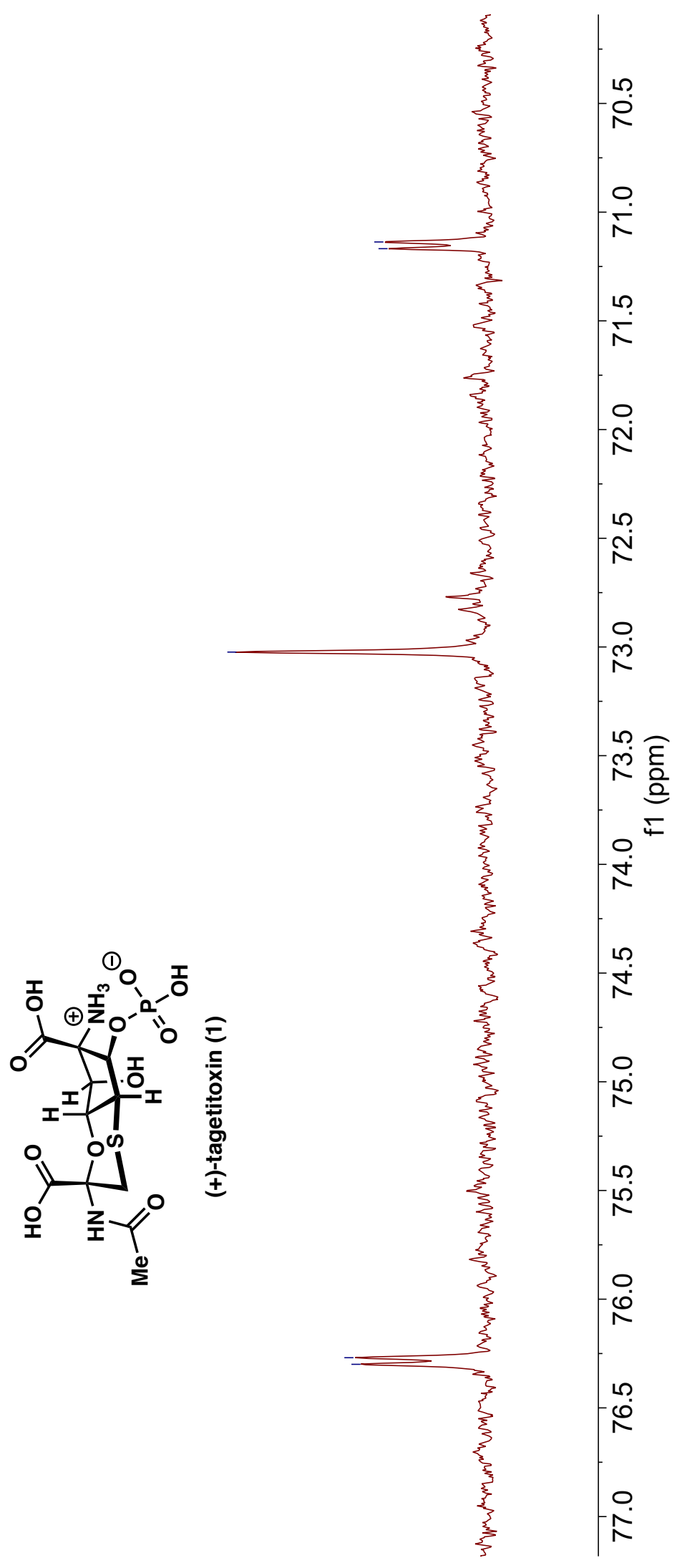


${ }^{13} \mathrm{C}$ NMR of $(+)-\mathbf{1}$ (comparison with $\mathrm{NH}_{4} \mathrm{HCO}_{3}$ )

$18 \cdot 22-$

$\varepsilon 9^{\circ} \varepsilon \varepsilon-$

$6 G^{\circ} \varepsilon b$

เ9. $\varepsilon \succ^{\top}$

$\nabla L \cdot L$

$\angle L \cdot L L$

Z० $\varepsilon L-$

$\angle Z^{\prime} 9 L-1$

$0 \varepsilon^{\circ} 9 L$

06.62

$9 \mathrm{CZ}^{\circ} \mathrm{s}$

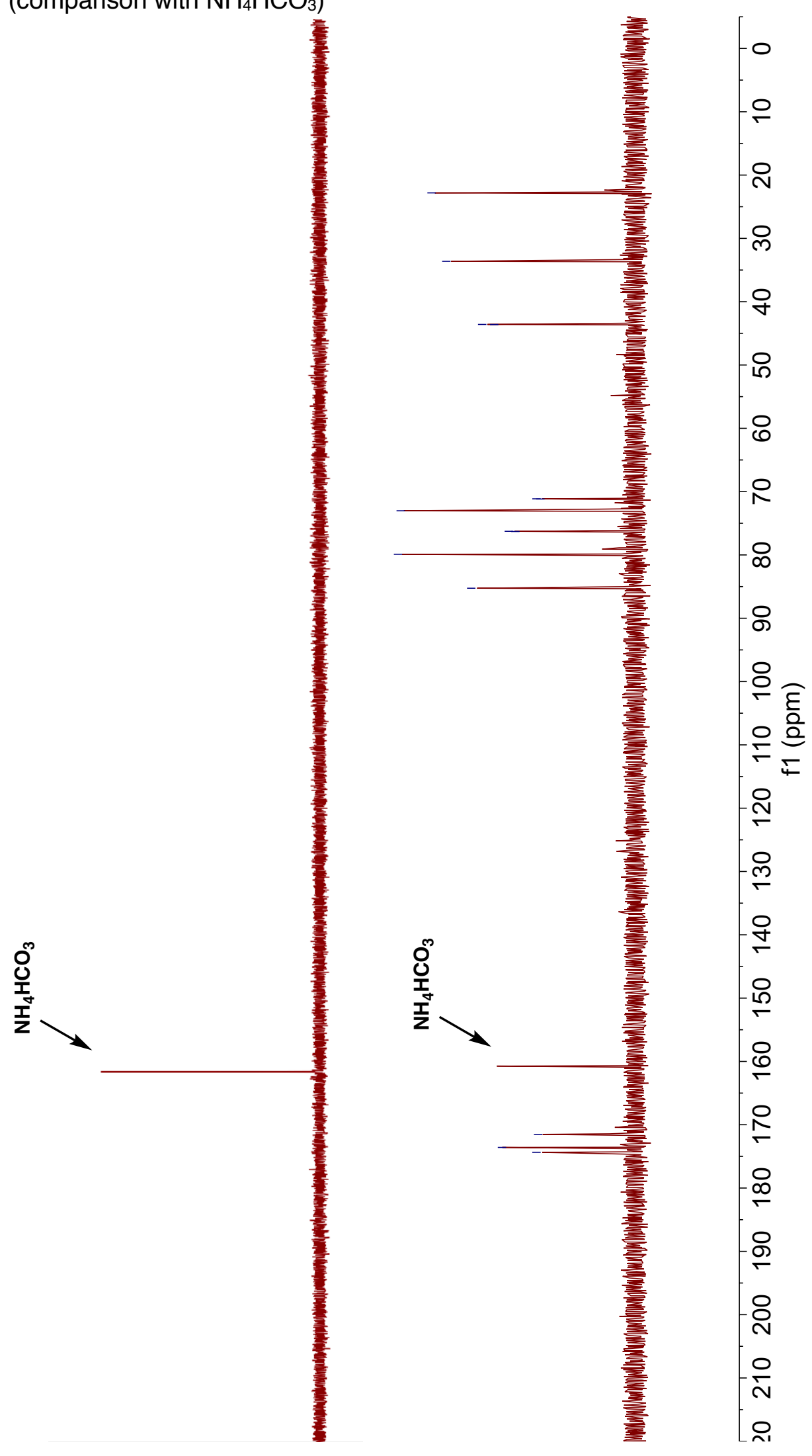

ZG'LLL

$89^{\circ} \varepsilon \angle L$

$9 \varepsilon^{\circ} \triangleright \angle L$ 
${ }^{31} \mathrm{P}$ NMR of $(+)-1\left(162 \mathrm{MHz}, 0.05 \mathrm{M} \mathrm{NH}_{4} \mathrm{HCO}_{3}\right.$ in $\left.\mathrm{D}_{2} \mathrm{O}\right)$

\section{$9 r^{\circ}+$}
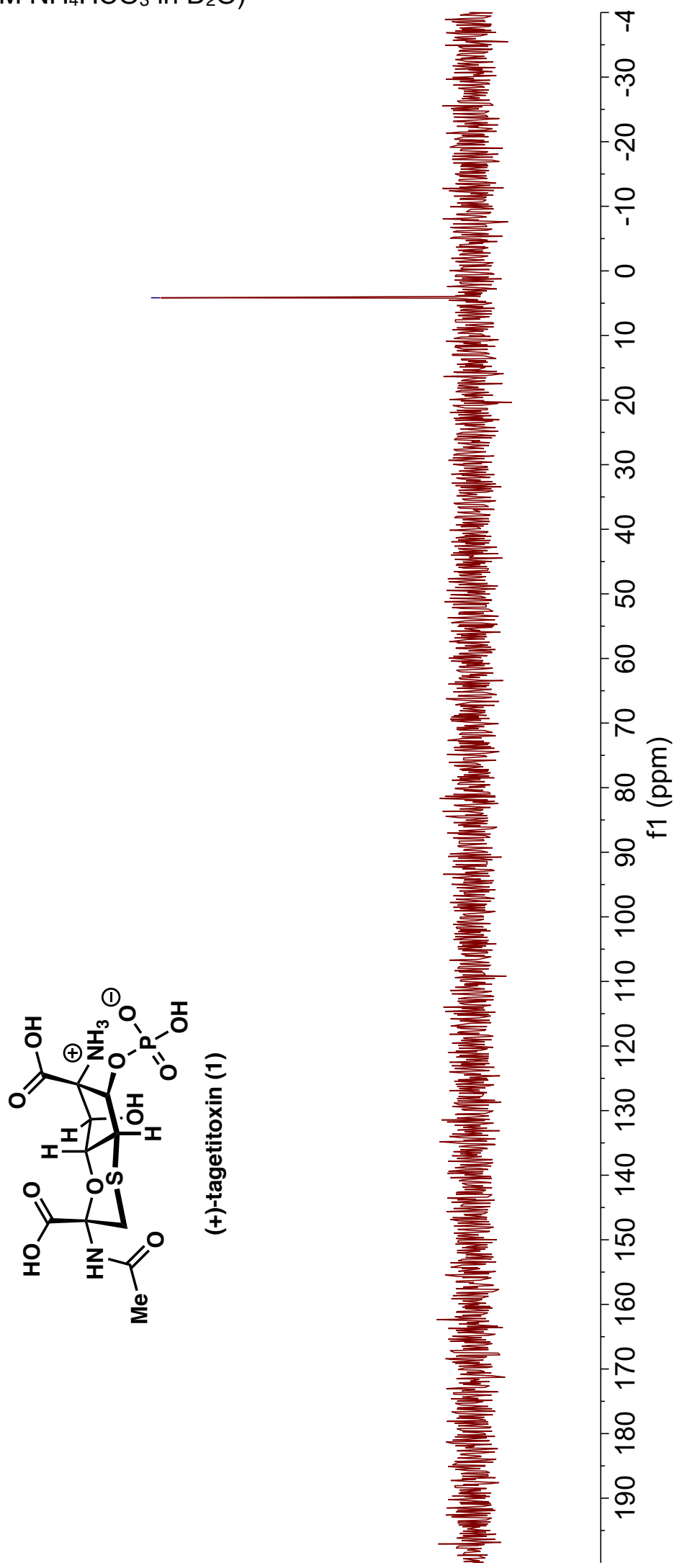


\section{VIII) X-ray Crystallography Data}

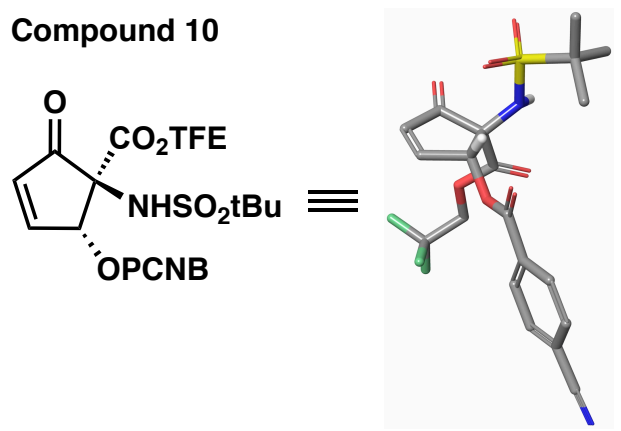

Table crystal-1-1. Crystal data and structure refinement for Baran732.

$\begin{array}{ll}\text { Report date } & 2019-07-17 \\ \text { Identification code } & \text { baran732 } \\ \text { Empirical formula } & \mathrm{C} 20 \mathrm{H} 19 \mathrm{~F} 3 \mathrm{~N} 2 \mathrm{O} 7 \mathrm{~S} \\ \text { Molecular formula } & \mathrm{C} 20 \mathrm{H} 19 \mathrm{~F} 3 \mathrm{~N} 2 \mathrm{O} 7 \mathrm{~S} \\ \text { Formula weight } & 488.43 \\ \text { Temperature } & 100.0 \mathrm{~K} \\ \text { Wavelength } & 1.54178 \AA \\ \text { Crystal system } & \text { Triclinic } \\ \text { Space group } & \mathrm{P}-1 \\ \text { Unit cell dimensions } & \mathrm{a}=8.8310(2) \AA \\ & \mathrm{b}=8.9849(2) \AA \\ & \mathrm{c}=14.0676(3) \AA \\ \text { Volume } & 1083.68(4) \AA^{3} \\ \text { Z } & 2 \\ \text { Density (calculated) } & 1.497 \mathrm{Mg} / \mathrm{m}^{3} \\ \text { Absorption coefficient } & 1.984 \mathrm{~mm}-1 \\ \text { F(000) } & 504 \\ \text { Crystal size } & 0.2 \times 0.115 \times 0.045 \mathrm{~mm}^{3} \\ \text { Crystal color, habit } & \text { colorless block } \\ \text { Theta range for data collection } & 3.234 \text { to } 70.143^{\circ} . \\ \text { Index ranges } & -10<=\mathrm{h}<=10,-10<=\mathrm{k}<=9,-17<=1<=17 \\ \text { Reflections collected } & 20519 \\ \text { Independent reflections } & 4015[\mathrm{R}(\mathrm{int})=0.0232] \\ \text { Completeness to theta }=67.500^{\circ} & 97.7 \% \\ \text { Absorption correction } & \text { Semi-empirical from equivalents } \\ & \\ & \end{array}$


Max. and min. transmission

Refinement method

Data / restraints / parameters

Goodness-of-fit on $\mathrm{F}^{2}$

Final $\mathrm{R}$ indices [I $>2 \operatorname{sigma}(\mathrm{I})]$

$\mathrm{R}$ indices (all data)

Extinction coefficient

Largest diff. peak and hole
0.7533 and 0.6274

Full-matrix least-squares on $\mathrm{F}^{2}$

4015 / 1 / 305

1.028

$\mathrm{R} 1=0.0268, \mathrm{wR} 2=0.0679$

$\mathrm{R} 1=0.0278, \mathrm{wR} 2=0.0687$

0.0006(2)

0.295 and -0.342 e. $\AA^{-3}$ 
Table crystal-1-2. Atomic coordinates ( $\left.\times 10^{4}\right)$ and equivalent isotropic displacement parameters $\left(\AA^{2} \times 10^{3}\right)$ for Baran732. $\mathrm{U}(\mathrm{eq})$ is defined as one third of the trace of the orthogonalized $\mathrm{U}^{\mathrm{ij}}$ tensor.

\begin{tabular}{|c|c|c|c|c|}
\hline & $\mathrm{x}$ & $\mathrm{y}$ & $\mathrm{z}$ & $\mathrm{U}(\mathrm{eq})$ \\
\hline$\overline{\mathrm{S}(1)}$ & $2795(1)$ & $7625(1)$ & $3882(1)$ & $16(1)$ \\
\hline $\mathrm{F}(1)$ & 9773(1) & 9796(1) & 1751(1) & $38(1)$ \\
\hline$F(2)$ & $11476(1)$ & $11074(1)$ & $2813(1)$ & $36(1)$ \\
\hline $\mathrm{F}(3)$ & $9292(1)$ & $11995(1)$ & $2617(1)$ & $33(1)$ \\
\hline $\mathrm{O}(1)$ & $4532(1)$ & $11157(1)$ & $3946(1)$ & $21(1)$ \\
\hline $\mathrm{O}(2)$ & $7700(1)$ & $8015(1)$ & $4128(1)$ & $22(1)$ \\
\hline $\mathrm{O}(3)$ & $7818(1)$ & $9757(1)$ & $3203(1)$ & $20(1)$ \\
\hline $\mathrm{O}(4)$ & $5022(1)$ & 4871(1) & $1130(1)$ & $54(1)$ \\
\hline $\mathrm{O}(5)$ & $6543(1)$ & 6904(1) & 1890(1) & $25(1)$ \\
\hline $\mathrm{O}(6)$ & $2245(1)$ & $7660(1)$ & 2901(1) & $21(1)$ \\
\hline $\mathrm{O}(7)$ & 2181(1) & $8663(1)$ & $4677(1)$ & $23(1)$ \\
\hline $\mathrm{N}(1)$ & 4651(1) & $7879(1)$ & $3943(1)$ & $18(1)$ \\
\hline $\mathrm{N}(2)$ & $12732(2)$ & $1612(2)$ & 299(1) & $39(1)$ \\
\hline $\mathrm{C}(1)$ & 7111(1) & $8733(1)$ & $3606(1)$ & $17(1)$ \\
\hline $\mathrm{C}(2)$ & 5411(1) & 8611(1) & $3265(1)$ & $18(1)$ \\
\hline$C(3)$ & $4853(1)$ & $10225(1)$ & $3233(1)$ & $19(1)$ \\
\hline$C(4)$ & 4781(1) & $10349(2)$ & 2211(1) & $26(1)$ \\
\hline$C(5)$ & $5047(1)$ & $9014(2)$ & $1635(1)$ & $28(1)$ \\
\hline$C(6)$ & $5224(1)$ & $7758(2)$ & $2170(1)$ & $23(1)$ \\
\hline$C(7)$ & 2487(1) & $5715(1)$ & $4067(1)$ & $19(1)$ \\
\hline$C(8)$ & 3043(1) & $5728(2)$ & $5122(1)$ & $25(1)$ \\
\hline$C(9)$ & $766(1)$ & 5374(2) & $3902(1)$ & $26(1)$ \\
\hline $\mathrm{C}(10)$ & $3338(2)$ & 4599(2) & $3329(1)$ & $26(1)$ \\
\hline $\mathrm{C}(11)$ & $6269(2)$ & $5456(2)$ & $1359(1)$ & $28(1)$ \\
\hline $\mathrm{C}(12)$ & $7710(2)$ & $4692(2)$ & $1109(1)$ & $26(1)$ \\
\hline$C(13)$ & $9134(2)$ & $5448(2)$ & $1353(1)$ & $24(1)$ \\
\hline$C(14)$ & $10438(2)$ & 4671(2) & $1122(1)$ & $25(1)$ \\
\hline$C(15)$ & $10305(2)$ & $3123(2)$ & $647(1)$ & $27(1)$ \\
\hline$C(16)$ & $8884(2)$ & $2370(2)$ & $387(1)$ & $33(1)$ \\
\hline $\mathrm{C}(17)$ & 7593(2) & $3150(2)$ & $620(1)$ & $32(1)$ \\
\hline$C(18)$ & $11665(2)$ & $2295(2)$ & $446(1)$ & $31(1)$ \\
\hline$C(19)$ & $9432(1)$ & $9964(2)$ & $3417(1)$ & $21(1)$ \\
\hline $\mathrm{C}(20)$ & 9991(1) & $10709(2)$ & $2645(1)$ & $23(1)$ \\
\hline
\end{tabular}


Table crystal-1-3. Bond lengths $[\AA]$ and angles $\left[^{\circ}\right]$ for Baran732.

\begin{tabular}{|c|c|}
\hline $\mathrm{S}(1)-\mathrm{O}(6)$ & $1.4412(9)$ \\
\hline $\mathrm{S}(1)-\mathrm{O}(7)$ & $1.4346(9)$ \\
\hline $\mathrm{S}(1)-\mathrm{N}(1)$ & $1.6412(10)$ \\
\hline $\mathrm{S}(1)-\mathrm{C}(7)$ & $1.8063(12)$ \\
\hline$F(1)-C(20)$ & $1.3362(15)$ \\
\hline$F(2)-C(20)$ & $1.3331(14)$ \\
\hline$F(3)-C(20)$ & $1.3367(15)$ \\
\hline $\mathrm{O}(1)-\mathrm{C}(3)$ & $1.2089(15)$ \\
\hline $\mathrm{O}(2)-\mathrm{C}(1)$ & $1.1900(15)$ \\
\hline $\mathrm{O}(3)-\mathrm{C}(1)$ & $1.3400(14)$ \\
\hline $\mathrm{O}(3)-\mathrm{C}(19)$ & $1.4336(13)$ \\
\hline $\mathrm{O}(4)-\mathrm{C}(11)$ & $1.1956(17)$ \\
\hline $\mathrm{O}(5)-\mathrm{C}(6)$ & $1.4454(15)$ \\
\hline $\mathrm{O}(5)-\mathrm{C}(11)$ & $1.3500(16)$ \\
\hline $\mathrm{N}(1)-\mathrm{H}(1)$ & $0.844(13)$ \\
\hline $\mathrm{N}(1)-\mathrm{C}(2)$ & $1.4584(15)$ \\
\hline $\mathrm{N}(2)-\mathrm{C}(18)$ & $1.148(2)$ \\
\hline$C(1)-C(2)$ & $1.5395(15)$ \\
\hline$C(2)-C(3)$ & $1.5577(17)$ \\
\hline$C(2)-C(6)$ & $1.5525(16)$ \\
\hline$C(3)-C(4)$ & $1.4639(17)$ \\
\hline $\mathrm{C}(4)-\mathrm{H}(4)$ & 0.9500 \\
\hline$C(4)-C(5)$ & $1.326(2)$ \\
\hline $\mathrm{C}(5)-\mathrm{H}(5)$ & 0.9500 \\
\hline$C(5)-C(6)$ & $1.4987(19)$ \\
\hline $\mathrm{C}(6)-\mathrm{H}(6)$ & 1.0000 \\
\hline$C(7)-C(8)$ & $1.5306(17)$ \\
\hline$C(7)-C(9)$ & $1.5319(16)$ \\
\hline$C(7)-C(10)$ & $1.5277(17)$ \\
\hline $\mathrm{C}(8)-\mathrm{H}(8 \mathrm{~A})$ & 0.9800 \\
\hline $\mathrm{C}(8)-\mathrm{H}(8 \mathrm{~B})$ & 0.9800 \\
\hline $\mathrm{C}(8)-\mathrm{H}(8 \mathrm{C})$ & 0.9800 \\
\hline $\mathrm{C}(9)-\mathrm{H}(9 \mathrm{~A})$ & 0.9800 \\
\hline $\mathrm{C}(9)-\mathrm{H}(9 \mathrm{~B})$ & 0.9800 \\
\hline $\mathrm{C}(9)-\mathrm{H}(9 \mathrm{C})$ & 0.9800 \\
\hline C(10)-H(10A) & 0.9800 \\
\hline $\mathrm{C}(10)-\mathrm{H}(10 \mathrm{~B})$ & 0.9800 \\
\hline C(10)-H(10C) & 0.9800 \\
\hline$C(11)-C(12)$ & $1.4924(19)$ \\
\hline$C(12)-C(13)$ & $1.3925(19)$ \\
\hline$C(12)-C(17)$ & $1.3974(19)$ \\
\hline $\mathrm{C}(13)-\mathrm{H}(13)$ & 0.9500 \\
\hline$C(13)-C(14)$ & $1.3867(19)$ \\
\hline $\mathrm{C}(14)-\mathrm{H}(14)$ & 0.9500 \\
\hline$C(14)-C(15)$ & $1.3971(19)$ \\
\hline$C(15)-C(16)$ & $1.391(2)$ \\
\hline
\end{tabular}




\begin{tabular}{|c|c|}
\hline$C(15)-C(18)$ & $1.445(2)$ \\
\hline $\mathrm{C}(16)-\mathrm{H}(16)$ & 0.9500 \\
\hline$C(16)-C(17)$ & $1.379(2)$ \\
\hline $\mathrm{C}(17)-\mathrm{H}(17)$ & 0.9500 \\
\hline $\mathrm{C}(19)-\mathrm{H}(19 \mathrm{~A})$ & 0.9900 \\
\hline C(19)-H(19B) & 0.9900 \\
\hline$C(19)-C(20)$ & $1.4987(17)$ \\
\hline $\mathrm{O}(6)-\mathrm{S}(1)-\mathrm{N}(1)$ & $106.88(5)$ \\
\hline $\mathrm{O}(6)-\mathrm{S}(1)-\mathrm{C}(7)$ & $109.37(5)$ \\
\hline $\mathrm{O}(7)-\mathrm{S}(1)-\mathrm{O}(6)$ & $117.81(5)$ \\
\hline $\mathrm{O}(7)-\mathrm{S}(1)-\mathrm{N}(1)$ & $110.43(5)$ \\
\hline $\mathrm{O}(7)-\mathrm{S}(1)-\mathrm{C}(7)$ & $107.53(5)$ \\
\hline $\mathrm{N}(1)-\mathrm{S}(1)-\mathrm{C}(7)$ & $103.95(5)$ \\
\hline $\mathrm{C}(1)-\mathrm{O}(3)-\mathrm{C}(19)$ & $115.86(9)$ \\
\hline $\mathrm{C}(11)-\mathrm{O}(5)-\mathrm{C}(6)$ & $116.25(10)$ \\
\hline $\mathrm{S}(1)-\mathrm{N}(1)-\mathrm{H}(1)$ & $110.6(10)$ \\
\hline$C(2)-N(1)-S(1)$ & $121.65(8)$ \\
\hline $\mathrm{C}(2)-\mathrm{N}(1)-\mathrm{H}(1)$ & $113.9(10)$ \\
\hline $\mathrm{O}(2)-\mathrm{C}(1)-\mathrm{O}(3)$ & $125.87(10)$ \\
\hline $\mathrm{O}(2)-\mathrm{C}(1)-\mathrm{C}(2)$ & $125.39(11)$ \\
\hline $\mathrm{O}(3)-\mathrm{C}(1)-\mathrm{C}(2)$ & $108.73(9)$ \\
\hline $\mathrm{N}(1)-\mathrm{C}(2)-\mathrm{C}(1)$ & $105.65(9)$ \\
\hline $\mathrm{N}(1)-\mathrm{C}(2)-\mathrm{C}(3)$ & $114.27(9)$ \\
\hline $\mathrm{N}(1)-\mathrm{C}(2)-\mathrm{C}(6)$ & $116.08(10)$ \\
\hline$C(1)-C(2)-C(3)$ & 109.92(9) \\
\hline$C(1)-C(2)-C(6)$ & $108.42(9)$ \\
\hline$C(6)-C(2)-C(3)$ & $102.40(9)$ \\
\hline $\mathrm{O}(1)-\mathrm{C}(3)-\mathrm{C}(2)$ & $123.86(10)$ \\
\hline $\mathrm{O}(1)-\mathrm{C}(3)-\mathrm{C}(4)$ & $128.55(12)$ \\
\hline$C(4)-C(3)-C(2)$ & $107.59(10)$ \\
\hline $\mathrm{C}(3)-\mathrm{C}(4)-\mathrm{H}(4)$ & 125.0 \\
\hline$C(5)-C(4)-C(3)$ & $109.92(12)$ \\
\hline $\mathrm{C}(5)-\mathrm{C}(4)-\mathrm{H}(4)$ & 125.0 \\
\hline $\mathrm{C}(4)-\mathrm{C}(5)-\mathrm{H}(5)$ & 123.3 \\
\hline$C(4)-C(5)-C(6)$ & $113.49(11)$ \\
\hline $\mathrm{C}(6)-\mathrm{C}(5)-\mathrm{H}(5)$ & 123.3 \\
\hline $\mathrm{O}(5)-\mathrm{C}(6)-\mathrm{C}(2)$ & $111.09(9)$ \\
\hline $\mathrm{O}(5)-\mathrm{C}(6)-\mathrm{C}(5)$ & $111.10(10)$ \\
\hline $\mathrm{O}(5)-\mathrm{C}(6)-\mathrm{H}(6)$ & 110.2 \\
\hline $\mathrm{C}(2)-\mathrm{C}(6)-\mathrm{H}(6)$ & 110.2 \\
\hline$C(5)-C(6)-C(2)$ & $103.93(10)$ \\
\hline $\mathrm{C}(5)-\mathrm{C}(6)-\mathrm{H}(6)$ & 110.2 \\
\hline $\mathrm{C}(8)-\mathrm{C}(7)-\mathrm{S}(1)$ & $108.05(8)$ \\
\hline$C(8)-C(7)-C(9)$ & $110.80(10)$ \\
\hline$C(9)-C(7)-S(1)$ & $105.29(8)$ \\
\hline $\mathrm{C}(10)-\mathrm{C}(7)-\mathrm{S}(1)$ & $109.27(8)$ \\
\hline$C(10)-C(7)-C(8)$ & $111.74(10)$ \\
\hline $\mathrm{C}(10)-\mathrm{C}(7)-\mathrm{C}(9)$ & $111.43(10)$ \\
\hline
\end{tabular}




$\begin{array}{ll}\mathrm{C}(7)-\mathrm{C}(8)-\mathrm{H}(8 \mathrm{~A}) & 109.5 \\ \mathrm{C}(7)-\mathrm{C}(8)-\mathrm{H}(8 \mathrm{~B}) & 109.5 \\ \mathrm{C}(7)-\mathrm{C}(8)-\mathrm{H}(8 \mathrm{C}) & 109.5 \\ \mathrm{H}(8 \mathrm{~A})-\mathrm{C}(8)-\mathrm{H}(8 \mathrm{~B}) & 109.5 \\ \mathrm{H}(8 \mathrm{~A})-\mathrm{C}(8)-\mathrm{H}(8 \mathrm{C}) & 109.5 \\ \mathrm{H}(8 \mathrm{~B})-\mathrm{C}(8)-\mathrm{H}(8 \mathrm{C}) & 109.5 \\ \mathrm{C}(7)-\mathrm{C}(9)-\mathrm{H}(9 \mathrm{~A}) & 109.5 \\ \mathrm{C}(7)-\mathrm{C}(9)-\mathrm{H}(9 \mathrm{~B}) & 109.5 \\ \mathrm{C}(7)-\mathrm{C}(9)-\mathrm{H}(9 \mathrm{C}) & 109.5 \\ \mathrm{H}(9 \mathrm{~A})-\mathrm{C}(9)-\mathrm{H}(9 \mathrm{~B}) & 109.5 \\ \mathrm{H}(9 \mathrm{~A})-\mathrm{C}(9)-\mathrm{H}(9 \mathrm{C}) & 109.5 \\ \mathrm{H}(9 \mathrm{~B})-\mathrm{C}(9)-\mathrm{H}(9 \mathrm{C}) & 109.5 \\ \mathrm{C}(7)-\mathrm{C}(10)-\mathrm{H}(10 \mathrm{~A}) & 109.5 \\ \mathrm{C}(7)-\mathrm{C}(10)-\mathrm{H}(10 \mathrm{~B}) & 109.5 \\ \mathrm{C}(7)-\mathrm{C}(10)-\mathrm{H}(10 \mathrm{C}) & 109.5 \\ \mathrm{H}(10 \mathrm{~A})-\mathrm{C}(10)-\mathrm{H}(10 \mathrm{~B}) & 109.5 \\ \mathrm{H}(10 \mathrm{~A})-\mathrm{C}(10)-\mathrm{H}(10 \mathrm{C}) & 109.5 \\ \mathrm{H}(10 \mathrm{~B})-\mathrm{C}(10)-\mathrm{H}(10 \mathrm{C}) & 109.5 \\ \mathrm{O}(4)-\mathrm{C}(11)-\mathrm{O}(5) & 123.63(13) \\ \mathrm{O}(4)-\mathrm{C}(11)-\mathrm{C}(12) & 124.88(13) \\ \mathrm{O}(5)-\mathrm{C}(11)-\mathrm{C}(12) & 111.49(11) \\ \mathrm{C}(13)-\mathrm{C}(12)-\mathrm{C}(11) & 122.65(12) \\ \mathrm{C}(13)-\mathrm{C}(12)-\mathrm{C}(17) & 119.92(13) \\ \mathrm{C}(17)-\mathrm{C}(12)-\mathrm{C}(11) & 117.42(12) \\ \mathrm{C}(12)-\mathrm{C}(13)-\mathrm{H}(13) & 119.9 \\ \mathrm{C}(14)-\mathrm{C}(13)-\mathrm{C}(12) & 120.18(12) \\ \mathrm{C}(14)-\mathrm{C}(13)-\mathrm{H}(13) & 119.9 \\ \mathrm{C}(13)-\mathrm{C}(14)-\mathrm{H}(14) & 120.4 \\ \mathrm{C}(13)-\mathrm{C}(14)-\mathrm{C}(15) & 119.29(13) \\ \mathrm{C}(15)-\mathrm{C}(14)-\mathrm{H}(14) & 120.4 \\ \mathrm{C}(14)-\mathrm{C}(15)-\mathrm{C}(18) & 119.33(13) \\ \mathrm{C}(16)-\mathrm{C}(15)-\mathrm{C}(14) & 120.71(13) \\ \mathrm{C}(16)-\mathrm{C}(15)-\mathrm{C}(18) & 119.94(13) \\ \mathrm{C}(15)-\mathrm{C}(16)-\mathrm{H}(16) & 120.2 \\ \mathrm{C}(17)-\mathrm{C}(16)-\mathrm{C}(15) & 119.65(13) \\ \mathrm{C}(17)-\mathrm{C}(16)-\mathrm{H}(16) & 120.2 \\ \mathrm{C}(12)-\mathrm{C}(17)-\mathrm{H}(17) & 119.9 \\ \mathrm{C}(16)-\mathrm{C}(17)-\mathrm{C}(12) & 120.23(13) \\ \mathrm{C}(16)-\mathrm{C}(17)-\mathrm{H}(17) & 119.9 \\ \mathrm{~N}(2)-\mathrm{C}(18)-\mathrm{C}(15) & 178.33(15) \\ \mathrm{O}(3)-\mathrm{C}(19)-\mathrm{H}(19 \mathrm{~A}) & 110.8 \\ \mathrm{O}(3)-\mathrm{C}(19)-\mathrm{H}(19 \mathrm{~B}) & 110.8 \\ \mathrm{O}(3)-\mathrm{C}(19)-\mathrm{C}(20) & 104.89(9) \\ \mathrm{H}(19 \mathrm{~A})-\mathrm{C}(19)-\mathrm{H}(19 \mathrm{~B}) & 108.8 \\ \mathrm{C}(20)-\mathrm{C}(19)-\mathrm{H}(19 \mathrm{~A}) & 110.8 \\ \mathrm{C}(20)-\mathrm{C}(19)-\mathrm{H}(19 \mathrm{~B}) & 110.8 \\ \mathrm{~F}(1)-\mathrm{C}(20)-\mathrm{F}(3) & 106.85(11) \\ \mathrm{F}(2)-\mathrm{C}(20)-\mathrm{F}(1) & \\ & \end{array}$


$\mathrm{F}(2)-\mathrm{C}(20)-\mathrm{F}(3)$

107.46(10)

$\mathrm{F}(2)-\mathrm{C}(20)-\mathrm{C}(19)$

110.53(10)

$\mathrm{F}(3)-\mathrm{C}(20)-\mathrm{C}(19)$

112.10(10) 
Table crystal-1-4. Anisotropic displacement parameters $\left(\AA^{2} \times 10^{3}\right)$ for Baran732. The anisotropic displacement factor exponent takes the form: $-2 \pi^{2}\left[h^{2} a^{* 2} U^{11}+\ldots+2 h k a^{*} b^{*} U^{12}\right]$

\begin{tabular}{|c|c|c|c|c|c|c|}
\hline & $\mathrm{U}^{11}$ & $\mathrm{U}^{22}$ & $\mathrm{U}^{33}$ & $\mathrm{U}^{23}$ & $\mathrm{U}^{13}$ & $\mathrm{U}^{12}$ \\
\hline $\mathrm{S}(1)$ & $13(1)$ & $17(1)$ & $20(1)$ & $6(1)$ & $2(1)$ & $1(1)$ \\
\hline $\mathrm{F}(1)$ & $44(1)$ & $44(1)$ & $23(1)$ & $0(1)$ & $10(1)$ & $-7(1)$ \\
\hline $\mathrm{F}(2)$ & $17(1)$ & $41(1)$ & $52(1)$ & $15(1)$ & $6(1)$ & $-8(1)$ \\
\hline $\mathrm{F}(3)$ & $31(1)$ & $28(1)$ & $45(1)$ & $17(1)$ & $3(1)$ & 1(1) \\
\hline $\mathrm{O}(1)$ & $18(1)$ & $21(1)$ & $26(1)$ & $7(1)$ & $-1(1)$ & $2(1)$ \\
\hline $\mathrm{O}(2)$ & $18(1)$ & $23(1)$ & $29(1)$ & $11(1)$ & $1(1)$ & $2(1)$ \\
\hline $\mathrm{O}(3)$ & $12(1)$ & $26(1)$ & $25(1)$ & $11(1)$ & $1(1)$ & $0(1)$ \\
\hline $\mathrm{O}(4)$ & $32(1)$ & $43(1)$ & $72(1)$ & $-19(1)$ & $3(1)$ & $-10(1)$ \\
\hline $\mathrm{O}(5)$ & $21(1)$ & $26(1)$ & $25(1)$ & $-2(1)$ & $5(1)$ & $-1(1)$ \\
\hline $\mathrm{O}(6)$ & $16(1)$ & $25(1)$ & $24(1)$ & $10(1)$ & $1(1)$ & $1(1)$ \\
\hline $\mathrm{O}(7)$ & $19(1)$ & $22(1)$ & $28(1)$ & $3(1)$ & $5(1)$ & $4(1)$ \\
\hline $\mathrm{N}(1)$ & $14(1)$ & $21(1)$ & $19(1)$ & $7(1)$ & 1(1) & $-1(1)$ \\
\hline $\mathrm{N}(2)$ & $52(1)$ & $33(1)$ & $32(1)$ & $6(1)$ & $9(1)$ & $12(1)$ \\
\hline $\mathrm{C}(1)$ & $15(1)$ & $17(1)$ & $18(1)$ & $2(1)$ & $4(1)$ & $2(1)$ \\
\hline $\mathrm{C}(2)$ & $14(1)$ & $21(1)$ & $19(1)$ & $6(1)$ & $1(1)$ & $-1(1)$ \\
\hline $\mathrm{C}(3)$ & $10(1)$ & $24(1)$ & $24(1)$ & $10(1)$ & $-1(1)$ & $-2(1)$ \\
\hline $\mathrm{C}(4)$ & $19(1)$ & $38(1)$ & $27(1)$ & $17(1)$ & $2(1)$ & $4(1)$ \\
\hline $\mathrm{C}(5)$ & $19(1)$ & $48(1)$ & $20(1)$ & $12(1)$ & 1(1) & $1(1)$ \\
\hline $\mathrm{C}(6)$ & $17(1)$ & $31(1)$ & $21(1)$ & $2(1)$ & $2(1)$ & $0(1)$ \\
\hline$C(7)$ & $18(1)$ & $17(1)$ & $24(1)$ & $8(1)$ & $1(1)$ & $-2(1)$ \\
\hline $\mathrm{C}(8)$ & $24(1)$ & $26(1)$ & $26(1)$ & $12(1)$ & $0(1)$ & $-2(1)$ \\
\hline $\mathrm{C}(9)$ & $21(1)$ & $31(1)$ & $28(1)$ & $10(1)$ & 1(1) & $-8(1)$ \\
\hline$C(10)$ & $29(1)$ & $19(1)$ & $29(1)$ & $5(1)$ & $2(1)$ & $3(1)$ \\
\hline $\mathrm{C}(11)$ & $30(1)$ & $28(1)$ & $24(1)$ & $0(1)$ & $3(1)$ & $-6(1)$ \\
\hline $\mathrm{C}(12)$ & $34(1)$ & $24(1)$ & $18(1)$ & $2(1)$ & $3(1)$ & $-2(1)$ \\
\hline $\mathrm{C}(13)$ & $33(1)$ & $22(1)$ & $16(1)$ & $2(1)$ & $2(1)$ & $-1(1)$ \\
\hline$C(14)$ & $33(1)$ & $25(1)$ & $17(1)$ & $5(1)$ & $2(1)$ & $1(1)$ \\
\hline$C(15)$ & $42(1)$ & $24(1)$ & $16(1)$ & $5(1)$ & $5(1)$ & $5(1)$ \\
\hline$C(16)$ & $51(1)$ & $21(1)$ & $25(1)$ & $0(1)$ & $5(1)$ & $-1(1)$ \\
\hline $\mathrm{C}(17)$ & $40(1)$ & $26(1)$ & $27(1)$ & $-1(1)$ & $3(1)$ & $-7(1)$ \\
\hline $\mathrm{C}(18)$ & $48(1)$ & $25(1)$ & $20(1)$ & $5(1)$ & $6(1)$ & $5(1)$ \\
\hline$C(19)$ & 11(1) & $27(1)$ & $25(1)$ & $8(1)$ & 1(1) & $0(1)$ \\
\hline $\mathrm{C}(20)$ & $18(1)$ & $23(1)$ & $27(1)$ & $4(1)$ & $4(1)$ & $-2(1)$ \\
\hline
\end{tabular}


Table crystal-1-5. Hydrogen coordinates ( $\left.\mathrm{x} 10^{4}\right)$ and isotropic displacement parameters $\left(\AA^{2} \times 10^{3}\right)$ for Baran732.

\begin{tabular}{|c|c|c|c|c|}
\hline & $\mathrm{x}$ & $\mathrm{y}$ & $\mathrm{z}$ & $\mathrm{U}(\mathrm{eq})$ \\
\hline $\mathrm{H}(1)$ & $5004(16)$ & $8177(17)$ & $4530(10)$ & 22 \\
\hline $\mathrm{H}(4)$ & 4575 & 11253 & 1993 & 32 \\
\hline $\mathrm{H}(5)$ & 5119 & 8867 & 950 & 34 \\
\hline $\mathrm{H}(6)$ & 4290 & 7058 & 2043 & 28 \\
\hline $\mathrm{H}(8 \mathrm{~A})$ & 4117 & 6077 & 5236 & 37 \\
\hline $\mathrm{H}(8 \mathrm{~B})$ & 2923 & 4692 & 5232 & 37 \\
\hline $\mathrm{H}(8 \mathrm{C})$ & 2445 & 6422 & 5574 & 37 \\
\hline $\mathrm{H}(9 \mathrm{~A})$ & 248 & 6174 & 4337 & 39 \\
\hline $\mathrm{H}(9 \mathrm{~B})$ & 512 & 4380 & 4045 & 39 \\
\hline $\mathrm{H}(9 \mathrm{C})$ & 438 & 5350 & 3220 & 39 \\
\hline $\mathrm{H}(10 \mathrm{~A})$ & 2958 & 4626 & 2663 & 38 \\
\hline $\mathrm{H}(10 \mathrm{~B})$ & 3176 & 3562 & 3428 & 38 \\
\hline $\mathrm{H}(10 \mathrm{C})$ & 4427 & 4893 & 3421 & 38 \\
\hline $\mathrm{H}(13)$ & 9214 & 6499 & 1680 & 29 \\
\hline $\mathrm{H}(14)$ & 11411 & 5185 & 1284 & 30 \\
\hline $\mathrm{H}(16)$ & 8803 & 1324 & 52 & 40 \\
\hline $\mathrm{H}(17)$ & 6620 & 2638 & 447 & 39 \\
\hline $\mathrm{H}(19 \mathrm{~A})$ & 9893 & 8968 & 3385 & 25 \\
\hline $\mathrm{H}(19 \mathrm{~B})$ & 9680 & 10626 & 4078 & 25 \\
\hline
\end{tabular}




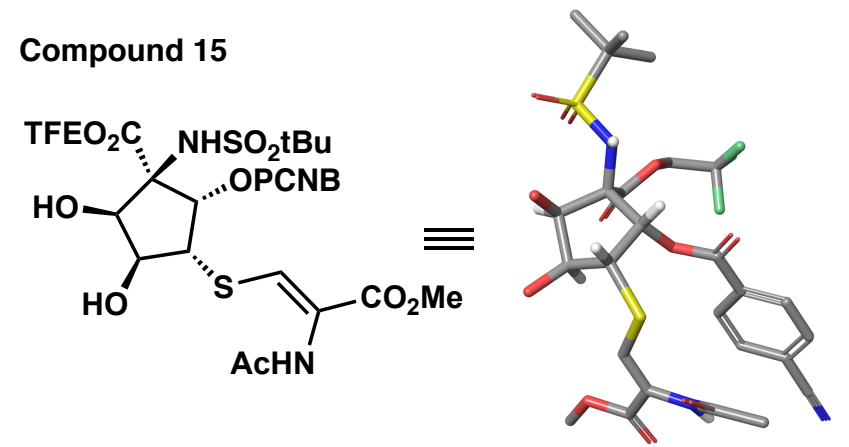

Table crystal-2-1. Crystal data and structure refinement for Baran738.

Report date

2019-09-13

Identification code

Baran 738

Empirical formula

C26 H30 F3 N3 O11 S2

Formula weight

681.65

Temperature

$100.15 \mathrm{~K}$

Wavelength

$1.54178 \AA$

Crystal system

Monoclinic

Space group

Unit cell dimensions

C $12 / \mathrm{c} 1$
$\mathrm{a}=26.165(4) \AA$
$\alpha=90^{\circ}$.
$\mathrm{b}=11.1380(15) \AA$
$\beta=110.448(6)^{\circ}$.
$\mathrm{c}=21.797(3) \AA$ $\gamma=90^{\circ}$.

Volume

$5952.0(15) \AA^{3}$

Z

8

Density (calculated)

$1.521 \mathrm{Mg} / \mathrm{m}^{3}$

Absorption coefficient

$2.373 \mathrm{~mm}^{-1}$

$\mathrm{F}(000)$

2832

Crystal size

$0.23 \times 0.11 \times 0.09 \mathrm{~mm}^{3}$

Theta range for data collection

3.606 to $72.542^{\circ}$.

Index ranges

$-32<=\mathrm{h}<=32,-13<=\mathrm{k}<=13,-26<=1<=26$

Reflections collected

79927

Independent reflections

$5873[\mathrm{R}(\mathrm{int})=0.0486]$

Completeness to theta $=67.679^{\circ}$

$100.0 \%$

Absorption correction

Semi-empirical from equivalents

Max. and min. transmission

0.7536 and 0.6517

Refinement method

Full-matrix least-squares on $\mathrm{F}^{2}$

Data / restraints / parameters

$5873 / 78 / 454$

Goodness-of-fit on $\mathrm{F}^{2}$ 
Final R indices [I $>2 \operatorname{sigma}(\mathrm{I})]$

$\mathrm{R} 1=0.0409, \mathrm{wR} 2=0.1098$

$\mathrm{R}$ indices (all data)

$\mathrm{R} 1=0.0438, \mathrm{wR} 2=0.1126$

Extinction coefficient

$\mathrm{n} / \mathrm{a}$

Largest diff. peak and hole

1.283 and -0.449 e. $\AA^{-3}$ 
Table crystal-2-2. Atomic coordinates ( $\left.\times 10^{4}\right)$ and equivalent isotropic displacement parameters $\left(\AA^{2} \times 10^{3}\right)$ for Baran738. U(eq) is defined as one third of the trace of the orthogonalized $U^{i j}$ tensor.

\begin{tabular}{|c|c|c|c|c|}
\hline & $\mathrm{x}$ & $\mathrm{y}$ & z & $\mathrm{U}(\mathrm{eq})$ \\
\hline$S(1)$ & 7091(1) & 7033(1) & $3258(1)$ & 19(1) \\
\hline$S(2)$ & $6858(1)$ & $2610(1)$ & $5170(1)$ & 19(1) \\
\hline $\mathrm{O}(1)$ & $6623(1)$ & $5508(1)$ & $5456(1)$ & $23(1)$ \\
\hline $\mathrm{O}(2)$ & $7220(1)$ & $6452(1)$ & $4749(1)$ & $22(1)$ \\
\hline $\mathrm{O}(3)$ & $7471(1)$ & 7771(1) & $3763(1)$ & $27(1)$ \\
\hline $\mathrm{O}(4)$ & $6524(1)$ & $7372(1)$ & $3048(1)$ & $27(1)$ \\
\hline $\mathrm{O}(5)$ & $5719(1)$ & $5492(1)$ & $3351(1)$ & $26(1)$ \\
\hline $\mathrm{O}(6)$ & $6153(1)$ & $4900(1)$ & 2671(1) & $23(1)$ \\
\hline $\mathrm{O}(7)$ & $6380(1)$ & $3159(1)$ & $3751(1)$ & $18(1)$ \\
\hline $\mathrm{O}(8)$ & $6952(1)$ & $1689(1)$ & $3671(1)$ & $29(1)$ \\
\hline $\mathrm{O}(9)$ & $4962(1)$ & $1396(1)$ & $5155(1)$ & $24(1)$ \\
\hline $\mathrm{O}(10)$ & $5248(1)$ & $3314(1)$ & $5330(1)$ & $23(1)$ \\
\hline $\mathrm{O}(11)$ & $6836(1)$ & $450(1)$ & $5804(1)$ & $22(1)$ \\
\hline $\mathrm{N}(1)$ & $7118(1)$ & $5631(1)$ & $3511(1)$ & $16(1)$ \\
\hline $\mathrm{N}(2)$ & $4450(1)$ & $-2053(2)$ & $3267(1)$ & $41(1)$ \\
\hline $\mathrm{N}(3)$ & $5945(1)$ & $627(2)$ & $5150(1)$ & $20(1)$ \\
\hline$C(1)$ & 6701(1) & $5248(2)$ & $3782(1)$ & $15(1)$ \\
\hline$C(2)$ & $6843(1)$ & $3939(2)$ & 4061(1) & $16(1)$ \\
\hline$C(3)$ & 6931(1) & $4038(2)$ & $4799(1)$ & $16(1)$ \\
\hline$C(4)$ & $6560(1)$ & $5086(2)$ & $4823(1)$ & $18(1)$ \\
\hline$C(5)$ & $6692(1)$ & $6009(2)$ & $4382(1)$ & $17(1)$ \\
\hline$C(6)$ & $7329(1)$ & $6987(2)$ & $2573(1)$ & $26(1)$ \\
\hline$C(7)$ & 7914(1) & $6511(2)$ & 2821(1) & $32(1)$ \\
\hline$C(8)$ & $7305(1)$ & $8291(2)$ & $2346(1)$ & $47(1)$ \\
\hline$C(9)$ & 6941(1) & $6215(2)$ & $2027(1)$ & $30(1)$ \\
\hline$C(10)$ & $6129(1)$ & $5252(2)$ & $3250(1)$ & $19(1)$ \\
\hline$C(11)$ & $5652(1)$ & 4917(2) & 2124(1) & $36(1)$ \\
\hline$C(13)$ & $6507(1)$ & $2004(2)$ & $3653(1)$ & $20(1)$ \\
\hline$C(14)$ & $6032(1)$ & $1176(2)$ & $3524(1)$ & $20(1)$ \\
\hline$C(15)$ & $5531(1)$ & $1559(2)$ & $3546(1)$ & $23(1)$ \\
\hline$C(16)$ & $5116(1)$ & $736(2)$ & $3471(1)$ & $26(1)$ \\
\hline
\end{tabular}




\begin{tabular}{lllll}
$\mathrm{C}(17)$ & $5203(1)$ & $-470(2)$ & $3371(1)$ & $23(1)$ \\
$\mathrm{C}(18)$ & $5700(1)$ & $-858(2)$ & $3339(1)$ & $26(1)$ \\
$\mathrm{C}(19)$ & $6114(1)$ & $-30(2)$ & $3414(1)$ & $25(1)$ \\
$\mathrm{C}(20)$ & $4777(1)$ & $-1344(2)$ & $3307(1)$ & $28(1)$ \\
$\mathrm{C}(21)$ & $6221(1)$ & $2751(2)$ & $5242(1)$ & $18(1)$ \\
$\mathrm{C}(22)$ & $5872(1)$ & $1860(2)$ & $5237(1)$ & $18(1)$ \\
$\mathrm{C}(23)$ & $5317(1)$ & $2146(2)$ & $5244(1)$ & $19(1)$ \\
$\mathrm{C}(24)$ & $3710(1)$ & $3661(2)$ & $5317(1)$ & $28(1)$ \\
$\mathrm{C}(25)$ & $-1(2)$ & $5420(1)$ & $20(1)$ \\
$\mathrm{C}(26)$ & $6415(1)$ & $-1292(2)$ & $5207(1)$ & $34(1)$ \\
$\mathrm{F}(1)$ & $6391(1)$ & $3979(4)$ & $1465(1)$ & $65(1)$ \\
$\mathrm{F}(2)$ & $6074(1)$ & $3785(8)$ & $1196(2)$ & $35(1)$ \\
$\mathrm{F}(3)$ & $5211(2)$ & $2835(2)$ & $2018(2)$ & $65(1)$ \\
$\mathrm{C}(12)$ & $5755(1)$ & $3885(3)$ & $1707(2)$ & $28(1)$ \\
$\mathrm{F}(1 \mathrm{~A})$ & $5680(1)$ & $3367(14)$ & $1672(6)$ & $60(4)$ \\
$\mathrm{F}(2 \mathrm{~A})$ & $5966(5)$ & $3860(40)$ & $1197(11)$ & $45(5)$ \\
$\mathrm{F}(3 \mathrm{~A})$ & $5098(9)$ & $3115(12)$ & $2193(5)$ & $61(3)$ \\
$\mathrm{C}(12 \mathrm{~A})$ & $5456(6)$ & $3821(8)$ & $1742(5)$ & $28(1)$ \\
& $5529(5)$ & & & \\
\hline
\end{tabular}


Table crystal-2-3. Bond lengths $[\AA]$ and angles $\left[{ }^{\circ}\right]$ for Baran 738 .

\begin{tabular}{|c|c|}
\hline $\mathrm{S}(1)-\mathrm{O}(3)$ & $1.4523(15)$ \\
\hline $\mathrm{S}(1)-\mathrm{O}(4)$ & $1.4418(15)$ \\
\hline $\mathrm{S}(1)-\mathrm{N}(1)$ & $1.6490(16)$ \\
\hline$S(1)-C(6)$ & $1.808(2)$ \\
\hline$S(2)-C(3)$ & $1.8248(19)$ \\
\hline$S(2)-C(21)$ & $1.7342(19)$ \\
\hline $\mathrm{O}(1)-\mathrm{C}(4)$ & $1.412(2)$ \\
\hline $\mathrm{O}(2)-\mathrm{C}(5)$ & $1.420(2)$ \\
\hline $\mathrm{O}(5)-\mathrm{C}(10)$ & $1.199(2)$ \\
\hline $\mathrm{O}(6)-\mathrm{C}(10)$ & $1.343(2)$ \\
\hline $\mathrm{O}(6)-\mathrm{C}(11)$ & $1.432(2)$ \\
\hline $\mathrm{O}(7)-\mathrm{C}(2)$ & $1.450(2)$ \\
\hline $\mathrm{O}(7)-\mathrm{C}(13)$ & $1.365(2)$ \\
\hline $\mathrm{O}(8)-\mathrm{C}(13)$ & $1.202(2)$ \\
\hline $\mathrm{O}(9)-\mathrm{C}(23)$ & $1.214(2)$ \\
\hline $\mathrm{O}(10)-\mathrm{C}(23)$ & $1.337(2)$ \\
\hline $\mathrm{O}(10)-\mathrm{C}(24)$ & $1.448(2)$ \\
\hline $\mathrm{O}(11)-\mathrm{C}(25)$ & $1.233(2)$ \\
\hline $\mathrm{N}(1)-\mathrm{C}(1)$ & $1.476(2)$ \\
\hline $\mathrm{N}(2)-\mathrm{C}(20)$ & $1.146(3)$ \\
\hline $\mathrm{N}(3)-\mathrm{C}(22)$ & $1.408(2)$ \\
\hline $\mathrm{N}(3)-\mathrm{C}(25)$ & $1.358(3)$ \\
\hline$C(1)-C(2)$ & $1.574(2)$ \\
\hline$C(1)-C(5)$ & $1.566(2)$ \\
\hline$C(1)-C(10)$ & $1.541(2)$ \\
\hline$C(2)-C(3)$ & $1.546(2)$ \\
\hline$C(3)-C(4)$ & $1.530(3)$ \\
\hline$C(4)-C(5)$ & $1.526(3)$ \\
\hline$C(6)-C(7)$ & $1.530(3)$ \\
\hline$C(6)-C(8)$ & $1.529(3)$ \\
\hline$C(6)-C(9)$ & $1.530(3)$ \\
\hline$C(11)-C(12)$ & $1.485(4)$ \\
\hline$C(11)-C(12 A)$ & $1.450(9)$ \\
\hline$C(13)-C(14)$ & $1.493(3)$ \\
\hline
\end{tabular}




\begin{tabular}{|c|c|}
\hline$C(14)-C(15)$ & $1.396(3)$ \\
\hline$C(14)-C(19)$ & $1.394(3)$ \\
\hline$C(15)-C(16)$ & $1.387(3)$ \\
\hline$C(16)-C(17)$ & $1.392(3)$ \\
\hline$C(17)-C(18)$ & $1.395(3)$ \\
\hline$C(17)-C(20)$ & $1.450(3)$ \\
\hline C(18)-C(19) & $1.388(3)$ \\
\hline$C(21)-C(22)$ & $1.346(3)$ \\
\hline$C(22)-C(23)$ & $1.493(3)$ \\
\hline$C(25)-C(26)$ & $1.506(3)$ \\
\hline $\mathrm{F}(1)-\mathrm{C}(12)$ & $1.314(3)$ \\
\hline $\mathrm{F}(2)-\mathrm{C}(12)$ & $1.343(3)$ \\
\hline $\mathrm{F}(3)-\mathrm{C}(12)$ & $1.333(3)$ \\
\hline $\mathrm{F}(1 \mathrm{~A})-\mathrm{C}(12 \mathrm{~A})$ & $1.309(7)$ \\
\hline $\mathrm{F}(2 \mathrm{~A})-\mathrm{C}(12 \mathrm{~A})$ & $1.321(8)$ \\
\hline $\mathrm{F}(3 \mathrm{~A})-\mathrm{C}(12 \mathrm{~A})$ & $1.324(8)$ \\
\hline $\mathrm{O}(3)-\mathrm{S}(1)-\mathrm{N}(1)$ & $110.14(8)$ \\
\hline $\mathrm{O}(3)-\mathrm{S}(1)-\mathrm{C}(6)$ & $107.50(10)$ \\
\hline $\mathrm{O}(4)-\mathrm{S}(1)-\mathrm{O}(3)$ & $116.63(9)$ \\
\hline $\mathrm{O}(4)-\mathrm{S}(1)-\mathrm{N}(1)$ & $105.91(8)$ \\
\hline $\mathrm{O}(4)-\mathrm{S}(1)-\mathrm{C}(6)$ & $110.96(10)$ \\
\hline $\mathrm{N}(1)-\mathrm{S}(1)-\mathrm{C}(6)$ & $105.13(9)$ \\
\hline $\mathrm{C}(21)-\mathrm{S}(2)-\mathrm{C}(3)$ & $102.30(9)$ \\
\hline $\mathrm{C}(10)-\mathrm{O}(6)-\mathrm{C}(11)$ & $116.51(16)$ \\
\hline $\mathrm{C}(13)-\mathrm{O}(7)-\mathrm{C}(2)$ & $115.36(14)$ \\
\hline $\mathrm{C}(23)-\mathrm{O}(10)-\mathrm{C}(24)$ & $115.84(15)$ \\
\hline $\mathrm{C}(1)-\mathrm{N}(1)-\mathrm{S}(1)$ & $117.09(12)$ \\
\hline $\mathrm{C}(25)-\mathrm{N}(3)-\mathrm{C}(22)$ & $126.05(17)$ \\
\hline $\mathrm{N}(1)-\mathrm{C}(1)-\mathrm{C}(2)$ & $108.35(14)$ \\
\hline $\mathrm{N}(1)-\mathrm{C}(1)-\mathrm{C}(5)$ & $114.04(14)$ \\
\hline $\mathrm{N}(1)-\mathrm{C}(1)-\mathrm{C}(10)$ & $110.95(15)$ \\
\hline$C(5)-C(1)-C(2)$ & $104.43(14)$ \\
\hline$C(10)-C(1)-C(2)$ & $109.25(14)$ \\
\hline$C(10)-C(1)-C(5)$ & $109.53(14)$ \\
\hline $\mathrm{O}(7)-\mathrm{C}(2)-\mathrm{C}(1)$ & $109.20(14)$ \\
\hline
\end{tabular}




\begin{tabular}{|c|c|}
\hline $\mathrm{O}(7)-\mathrm{C}(2)-\mathrm{C}(3)$ & $108.91(14)$ \\
\hline$C(3)-C(2)-C(1)$ & $105.35(14)$ \\
\hline$C(2)-C(3)-S(2)$ & $113.46(13)$ \\
\hline$C(4)-C(3)-S(2)$ & $118.98(13)$ \\
\hline$C(4)-C(3)-C(2)$ & $102.98(14)$ \\
\hline $\mathrm{O}(1)-\mathrm{C}(4)-\mathrm{C}(3)$ & $115.37(15)$ \\
\hline $\mathrm{O}(1)-\mathrm{C}(4)-\mathrm{C}(5)$ & $114.61(15)$ \\
\hline$C(5)-C(4)-C(3)$ & $101.95(14)$ \\
\hline $\mathrm{O}(2)-\mathrm{C}(5)-\mathrm{C}(1)$ & $111.29(14)$ \\
\hline $\mathrm{O}(2)-\mathrm{C}(5)-\mathrm{C}(4)$ & $105.59(15)$ \\
\hline$C(4)-C(5)-C(1)$ & $103.50(14)$ \\
\hline$C(7)-C(6)-S(1)$ & $107.98(14)$ \\
\hline$C(7)-C(6)-C(9)$ & $112.98(19)$ \\
\hline$C(8)-C(6)-S(1)$ & $104.69(16)$ \\
\hline$C(8)-C(6)-C(7)$ & $111.4(2)$ \\
\hline $\mathrm{C}(8)-\mathrm{C}(6)-\mathrm{C}(9)$ & 109.99(19) \\
\hline$C(9)-C(6)-S(1)$ & $109.40(15)$ \\
\hline $\mathrm{O}(5)-\mathrm{C}(10)-\mathrm{O}(6)$ & $125.28(18)$ \\
\hline $\mathrm{O}(5)-\mathrm{C}(10)-\mathrm{C}(1)$ & $123.95(17)$ \\
\hline $\mathrm{O}(6)-\mathrm{C}(10)-\mathrm{C}(1)$ & $110.71(15)$ \\
\hline $\mathrm{O}(6)-\mathrm{C}(11)-\mathrm{C}(12)$ & $105.8(2)$ \\
\hline $\mathrm{O}(6)-\mathrm{C}(11)-\mathrm{C}(12 \mathrm{~A})$ & $115.0(5)$ \\
\hline $\mathrm{O}(7)-\mathrm{C}(13)-\mathrm{C}(14)$ & $112.27(16)$ \\
\hline $\mathrm{O}(8)-\mathrm{C}(13)-\mathrm{O}(7)$ & $123.75(18)$ \\
\hline $\mathrm{O}(8)-\mathrm{C}(13)-\mathrm{C}(14)$ & $123.97(18)$ \\
\hline$C(15)-C(14)-C(13)$ & $122.40(18)$ \\
\hline$C(19)-C(14)-C(13)$ & $117.36(18)$ \\
\hline$C(19)-C(14)-C(15)$ & $120.15(18)$ \\
\hline$C(16)-C(15)-C(14)$ & $120.04(19)$ \\
\hline$C(15)-C(16)-C(17)$ & $119.5(2)$ \\
\hline$C(16)-C(17)-C(18)$ & $120.92(19)$ \\
\hline$C(16)-C(17)-C(20)$ & $120.0(2)$ \\
\hline $\mathrm{C}(18)-\mathrm{C}(17)-\mathrm{C}(20)$ & $119.07(19)$ \\
\hline $\mathrm{C}(19)-\mathrm{C}(18)-\mathrm{C}(17)$ & $119.38(19)$ \\
\hline $\mathrm{C}(18)-\mathrm{C}(19)-\mathrm{C}(14)$ & $120.0(2)$ \\
\hline $\mathrm{N}(2)-\mathrm{C}(20)-\mathrm{C}(17)$ & $178.3(3)$ \\
\hline
\end{tabular}




$\begin{array}{ll}\mathrm{C}(22)-\mathrm{C}(21)-\mathrm{S}(2) & 127.08(16) \\ \mathrm{N}(3)-\mathrm{C}(22)-\mathrm{C}(23) & 112.75(16) \\ \mathrm{C}(21)-\mathrm{C}(22)-\mathrm{N}(3) & 126.63(18) \\ \mathrm{C}(21)-\mathrm{C}(22)-\mathrm{C}(23) & 120.22(18) \\ \mathrm{O}(9)-\mathrm{C}(23)-\mathrm{O}(10) & 123.91(18) \\ \mathrm{O}(9)-\mathrm{C}(23)-\mathrm{C}(22) & 123.12(18) \\ \mathrm{O}(10)-\mathrm{C}(23)-\mathrm{C}(22) & 112.92(16) \\ \mathrm{O}(11)-\mathrm{C}(25)-\mathrm{N}(3) & 122.91(18) \\ \mathrm{O}(11)-\mathrm{C}(25)-\mathrm{C}(26) & 121.94(18) \\ \mathrm{N}(3)-\mathrm{C}(25)-\mathrm{C}(26) & 115.13(17) \\ \mathrm{F}(1)-\mathrm{C}(12)-\mathrm{C}(11) & 113.5(2) \\ \mathrm{F}(1)-\mathrm{C}(12)-\mathrm{F}(2) & 107.0(3) \\ \mathrm{F}(1)-\mathrm{C}(12)-\mathrm{F}(3) & 106.1(3) \\ \mathrm{F}(2)-\mathrm{C}(12)-\mathrm{C}(11) & 110.2(4) \\ \mathrm{F}(3)-\mathrm{C}(12)-\mathrm{C}(11) & 113.4(3) \\ \mathrm{F}(3)-\mathrm{C}(12)-\mathrm{F}(2) & 106.1(4) \\ \mathrm{F}(1 \mathrm{~A})-\mathrm{C}(12 \mathrm{~A})-\mathrm{C}(11) & 111.4(9) \\ \mathrm{F}(1 \mathrm{~A})-\mathrm{C}(12 \mathrm{~A})-\mathrm{F}(2 \mathrm{~A}) & 114.5(16) \\ \mathrm{F}(1 \mathrm{~A})-\mathrm{C}(12 \mathrm{~A})-\mathrm{F}(3 \mathrm{~A}) & 102.5(12) \\ \mathrm{F}(2 \mathrm{~A})-\mathrm{C}(12 \mathrm{~A})-\mathrm{C}(11) & 116(2) \\ \mathrm{F}(2 \mathrm{~A})-\mathrm{C}(12 \mathrm{~A})-\mathrm{F}(3 \mathrm{~A}) & 112.9(19) \\ \mathrm{F}(3 \mathrm{~A})-\mathrm{C}(12 \mathrm{~A})-\mathrm{C}(11) & 97.7(8)\end{array}$

Symmetry transformations used to generate equivalent atoms: 
Table crystal-2-4. Anisotropic displacement parameters $\left(\AA^{2} \mathrm{x} 10^{3}\right)$ for Baran738. The anisotropic displacement factor exponent takes the form: $-2 \pi^{2}\left[h^{2} a^{* 2} U^{11}+\ldots+2 h k a^{*} b^{*} U^{12}\right]$

\begin{tabular}{|c|c|c|c|c|c|c|}
\hline & $\mathrm{U}^{11}$ & $\mathrm{U}^{22}$ & $\mathrm{U}^{33}$ & $U^{23}$ & $\mathrm{U}^{13}$ & $\mathrm{U}^{12}$ \\
\hline$S(1)$ & $24(1)$ & $13(1)$ & $19(1)$ & $2(1)$ & $8(1)$ & $0(1)$ \\
\hline$S(2)$ & $17(1)$ & $17(1)$ & $24(1)$ & $3(1)$ & $9(1)$ & $2(1)$ \\
\hline $\mathrm{O}(1)$ & $28(1)$ & $21(1)$ & $23(1)$ & $-4(1)$ & $13(1)$ & $-3(1)$ \\
\hline $\mathrm{O}(2)$ & $26(1)$ & $21(1)$ & $21(1)$ & $-4(1)$ & $8(1)$ & $-8(1)$ \\
\hline $\mathrm{O}(3)$ & $37(1)$ & $18(1)$ & $24(1)$ & $-1(1)$ & $9(1)$ & $-7(1)$ \\
\hline $\mathrm{O}(4)$ & $29(1)$ & 21(1) & $30(1)$ & $4(1)$ & $9(1)$ & $8(1)$ \\
\hline $\mathrm{O}(5)$ & $17(1)$ & $31(1)$ & $28(1)$ & $-3(1)$ & $7(1)$ & $2(1)$ \\
\hline $\mathrm{O}(6)$ & $18(1)$ & $28(1)$ & $18(1)$ & $-5(1)$ & $0(1)$ & $4(1)$ \\
\hline $\mathrm{O}(7)$ & $16(1)$ & $14(1)$ & $22(1)$ & $-2(1)$ & $5(1)$ & $-3(1)$ \\
\hline $\mathrm{O}(8)$ & $25(1)$ & $23(1)$ & $45(1)$ & $-9(1)$ & $20(1)$ & $-2(1)$ \\
\hline $\mathrm{O}(9)$ & $18(1)$ & $20(1)$ & $36(1)$ & $-2(1)$ & $10(1)$ & $-3(1)$ \\
\hline $\mathrm{O}(10)$ & $18(1)$ & $17(1)$ & $37(1)$ & $1(1)$ & $13(1)$ & 1(1) \\
\hline $\mathrm{O}(11)$ & $18(1)$ & $18(1)$ & $27(1)$ & $4(1)$ & $3(1)$ & $-1(1)$ \\
\hline $\mathrm{N}(1)$ & $14(1)$ & $14(1)$ & $18(1)$ & $3(1)$ & $6(1)$ & $0(1)$ \\
\hline $\mathrm{N}(2)$ & $45(1)$ & $37(1)$ & $34(1)$ & $3(1)$ & $3(1)$ & $-20(1)$ \\
\hline $\mathrm{N}(3)$ & $15(1)$ & $17(1)$ & $26(1)$ & $-3(1)$ & $5(1)$ & $-1(1)$ \\
\hline$C(1)$ & $14(1)$ & $14(1)$ & $17(1)$ & $0(1)$ & $6(1)$ & $-1(1)$ \\
\hline$C(2)$ & $15(1)$ & $14(1)$ & $19(1)$ & $-1(1)$ & $5(1)$ & $-3(1)$ \\
\hline$C(3)$ & $13(1)$ & $16(1)$ & $19(1)$ & $1(1)$ & $6(1)$ & $-2(1)$ \\
\hline$C(4)$ & $19(1)$ & $16(1)$ & $20(1)$ & $-2(1)$ & $8(1)$ & $0(1)$ \\
\hline$C(5)$ & $16(1)$ & $15(1)$ & $19(1)$ & $-2(1)$ & $6(1)$ & $-2(1)$ \\
\hline$C(6)$ & $34(1)$ & $25(1)$ & $20(1)$ & $4(1)$ & $12(1)$ & $-4(1)$ \\
\hline$C(7)$ & $30(1)$ & $41(1)$ & $30(1)$ & $0(1)$ & $16(1)$ & $-8(1)$ \\
\hline$C(8)$ & $74(2)$ & $32(1)$ & $39(1)$ & $14(1)$ & $26(1)$ & $-6(1)$ \\
\hline$C(9)$ & $31(1)$ & $37(1)$ & $19(1)$ & $-1(1)$ & $8(1)$ & $-1(1)$ \\
\hline$C(10)$ & $17(1)$ & $16(1)$ & $21(1)$ & $-1(1)$ & $4(1)$ & $0(1)$ \\
\hline $\mathrm{C}(11)$ & $25(1)$ & $44(1)$ & $26(1)$ & $-9(1)$ & $-7(1)$ & $10(1)$ \\
\hline$C(13)$ & $23(1)$ & $17(1)$ & $22(1)$ & $-3(1)$ & $10(1)$ & $-1(1)$ \\
\hline$C(14)$ & $24(1)$ & $17(1)$ & $18(1)$ & $-2(1)$ & $6(1)$ & $-3(1)$ \\
\hline$C(15)$ & $23(1)$ & $16(1)$ & $29(1)$ & $-1(1)$ & $7(1)$ & $-2(1)$ \\
\hline$C(16)$ & $22(1)$ & $23(1)$ & $30(1)$ & $1(1)$ & $7(1)$ & $-2(1)$ \\
\hline
\end{tabular}




\begin{tabular}{lllllll}
$\mathrm{C}(17)$ & $27(1)$ & $21(1)$ & $18(1)$ & $1(1)$ & $3(1)$ & $-7(1)$ \\
$\mathrm{C}(18)$ & $34(1)$ & $16(1)$ & $27(1)$ & $-3(1)$ & $8(1)$ & $-4(1)$ \\
$\mathrm{C}(19)$ & $28(1)$ & $19(1)$ & $28(1)$ & $-4(1)$ & $11(1)$ & $-2(1)$ \\
$\mathrm{C}(20)$ & $31(1)$ & $27(1)$ & $21(1)$ & $2(1)$ & $2(1)$ & $-8(1)$ \\
$\mathrm{C}(21)$ & $17(1)$ & $19(1)$ & $18(1)$ & $3(1)$ & $6(1)$ & $2(1)$ \\
$\mathrm{C}(22)$ & $18(1)$ & $17(1)$ & $18(1)$ & $1(1)$ & $7(1)$ & $1(1)$ \\
$\mathrm{C}(23)$ & $20(1)$ & $17(1)$ & $20(1)$ & $1(1)$ & $7(1)$ & $1(1)$ \\
$\mathrm{C}(24)$ & $20(1)$ & $21(1)$ & $47(1)$ & $0(1)$ & $16(1)$ & $4(1)$ \\
$\mathrm{C}(25)$ & $18(1)$ & $18(1)$ & $23(1)$ & $2(1)$ & $7(1)$ & $0(1)$ \\
$\mathrm{C}(26)$ & $23(1)$ & $21(1)$ & $50(1)$ & $-9(1)$ & $4(1)$ & $2(1)$ \\
$\mathrm{F}(1)$ & $40(1)$ & $116(2)$ & $44(1)$ & $-41(2)$ & $24(1)$ & $-26(1)$ \\
$\mathrm{F}(2)$ & $28(2)$ & $45(2)$ & $24(1)$ & $-6(1)$ & $0(1)$ & $-13(2)$ \\
$\mathrm{F}(3)$ & $74(2)$ & $25(1)$ & $63(2)$ & $-1(1)$ & $-18(2)$ & $-11(1)$ \\
$\mathrm{C}(12)$ & $14(2)$ & $32(1)$ & $32(1)$ & $-6(1)$ & $0(1)$ & $-6(1)$ \\
$\mathrm{F}(1 \mathrm{~A})$ & $36(5)$ & $81(10)$ & $49(6)$ & $-30(6)$ & $-2(3)$ & $29(5)$ \\
$\mathrm{F}(2 \mathrm{~A})$ & $22(6)$ & $52(8)$ & $41(5)$ & $-11(5)$ & $-14(5)$ & $-3(7)$ \\
$\mathrm{F}(3 \mathrm{~A})$ & $66(7)$ & $51(5)$ & $47(5)$ & $12(4)$ & $-3(4)$ & $-18(5)$ \\
$\mathrm{C}(12 \mathrm{~A})$ & $14(2)$ & $32(1)$ & $32(1)$ & $-6(1)$ & $0(1)$ & $-6(1)$ \\
& & & & & & \\
\hline
\end{tabular}


Table crystal-2-5. Hydrogen coordinates ( $\left.\times 10^{4}\right)$ and isotropic displacement parameters $\left(\AA^{2} \times 10^{3}\right)$ for Baran 738 .

\begin{tabular}{|c|c|c|c|c|}
\hline & $\mathrm{x}$ & $\mathrm{y}$ & z & $\mathrm{U}(\mathrm{eq})$ \\
\hline $\mathrm{H}(1)$ & 6951(11) & $5880(30)$ & 5611(13) & 34 \\
\hline $\mathrm{H}(2)$ & $7337(11)$ & $7050(30)$ & $4546(14)$ & 33 \\
\hline $\mathrm{H}(1 \mathrm{~A})$ & $7439(7)$ & $5430(20)$ & $3753(11)$ & 23 \\
\hline $\mathrm{H}(3)$ & $5651(8)$ & $210(20)$ & $4978(11)$ & 24 \\
\hline $\mathrm{H}(2 \mathrm{~A})$ & 7178 & 3632 & 3993 & 19 \\
\hline $\mathrm{H}(3 \mathrm{~A})$ & 7315 & 4312 & 5024 & 19 \\
\hline $\mathrm{H}(4)$ & 6173 & 4826 & 4606 & 22 \\
\hline $\mathrm{H}(5)$ & 6414 & 6666 & 4249 & 20 \\
\hline $\mathrm{H}(7 \mathrm{~A})$ & 8147 & 7060 & 3153 & 48 \\
\hline $\mathrm{H}(7 \mathrm{~B})$ & 8048 & 6454 & 2455 & 48 \\
\hline $\mathrm{H}(7 \mathrm{C})$ & 7922 & 5714 & 3015 & 48 \\
\hline $\mathrm{H}(8 \mathrm{~A})$ & 6930 & 8586 & 2218 & 70 \\
\hline $\mathrm{H}(8 \mathrm{~B})$ & 7429 & 8338 & 1971 & 70 \\
\hline $\mathrm{H}(8 \mathrm{C})$ & 7543 & 8785 & 2705 & 70 \\
\hline $\mathrm{H}(9 \mathrm{~A})$ & 6932 & 5398 & 2190 & 44 \\
\hline $\mathrm{H}(9 \mathrm{~B})$ & 7069 & 6191 & 1655 & 44 \\
\hline $\mathrm{H}(9 \mathrm{C})$ & 6574 & 6561 & 1887 & 44 \\
\hline $\mathrm{H}(11 \mathrm{~A})$ & 5613 & 5681 & 1880 & 43 \\
\hline $\mathrm{H}(11 \mathrm{~B})$ & 5337 & 4830 & 2274 & 43 \\
\hline $\mathrm{H}(11 \mathrm{C})$ & 5664 & 5589 & 1833 & 43 \\
\hline $\mathrm{H}(11 \mathrm{D})$ & 5350 & 5081 & 2288 & 43 \\
\hline $\mathrm{H}(15)$ & 5474 & 2384 & 3612 & 28 \\
\hline $\mathrm{H}(16)$ & 4774 & 993 & 3487 & 31 \\
\hline $\mathrm{H}(18)$ & 5754 & -1681 & 3265 & 31 \\
\hline $\mathrm{H}(19)$ & 6453 & -286 & 3392 & 30 \\
\hline $\mathrm{H}(21)$ & 6106 & 3543 & 5290 & 22 \\
\hline $\mathrm{H}(24 \mathrm{~A})$ & 4437 & 3414 & 4900 & 42 \\
\hline $\mathrm{H}(24 \mathrm{~B})$ & 4697 & 4535 & 5363 & 42 \\
\hline $\mathrm{H}(24 \mathrm{C})$ & 4634 & 3270 & 5678 & 42 \\
\hline $\mathrm{H}(26 \mathrm{~A})$ & 6649 & -1419 & 4978 & 51 \\
\hline $\mathrm{H}(26 \mathrm{~B})$ & 6021 & -1483 & 4913 & 51 \\
\hline $\mathrm{H}(26 \mathrm{C})$ & 6488 & -1816 & 5593 & 51 \\
\hline
\end{tabular}


Table crystal-2-6. Hydrogen bonds for Baran738 [ $\AA$ and $\left.{ }^{\circ}\right]$.

\begin{tabular}{lcccc}
\hline D-H...A & d(D-H) & d(H...A & $d(D \ldots A)$ & $<($ DHA $)$ \\
\hline $\mathrm{O}(2)-\mathrm{H}(2) \ldots \mathrm{O}(3)$ & $0.91(3)$ & $2.02(3)$ & $2.861(2)$ & $153(2)$ \\
$\mathrm{N}(1)-\mathrm{H}(1 \mathrm{~A}) \ldots \mathrm{O}(11) \# 1$ & $0.852(16)$ & $2.050(17)$ & $2.883(2)$ & $166(2)$ \\
& & & & \\
\hline
\end{tabular}

Symmetry transformations used to generate equivalent atoms:

$\# 1-x+3 / 2,-y+1 / 2,-z+1$ 


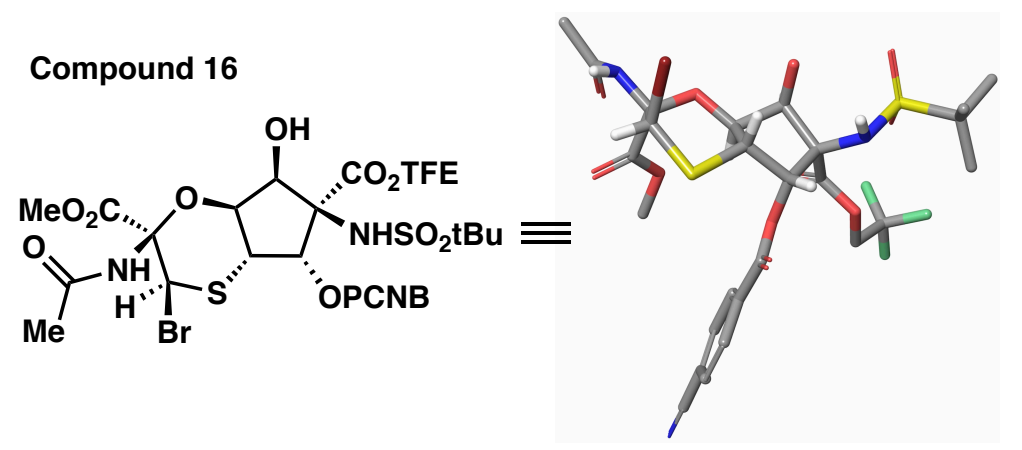

Table crystal-3-1. Crystal data and structure refinement for baran739_0m_a.

Identification code

Empirical formula

Formula weight

Temperature

Wavelength

Crystal system

Space group

Unit cell dimensions

Volume

$\mathrm{Z}$

Density (calculated)

Absorption coefficient

$\mathrm{F}(000)$

Crystal size

Theta range for data collection

Index ranges

Reflections collected

Independent reflections

Completeness to theta $=67.679^{\circ}$

Absorption correction

Max. and min. transmission

Refinement method

Data / restraints / parameters

Goodness-of-fit on $\mathrm{F}^{2}$

Final $\mathrm{R}$ indices [I $>2 \operatorname{sigma}(\mathrm{I})]$

$\mathrm{R}$ indices (all data)

Absolute structure parameter

Largest diff. peak and hole
HC-30215

C26 H29 Br F3 N3 O11 S2

760.55

$100.0 \mathrm{~K}$

$1.54178 \AA$

Monoclinic

P 1211

$$
\begin{array}{ll}
\mathrm{a}=8.8653(16) \AA & \alpha=90^{\circ} . \\
\mathrm{b}=11.016(3) \AA & \beta=99.307(10)^{\circ} . \\
\mathrm{c}=16.680(4) \AA & \gamma=90^{\circ} .
\end{array}
$$

$1607.5(6) \AA^{3}$

2

$1.571 \mathrm{Mg} / \mathrm{m}^{3}$

$3.664 \mathrm{~mm}^{-1}$

776

$0.29 \times 0.28 \times 0.04 \mathrm{~mm}^{3}$

2.684 to $69.040^{\circ}$.

$-10<=\mathrm{h}<=10,-13<=\mathrm{k}<=13,-20<=\mathrm{l}<=20$

23644

$5883[\mathrm{R}(\mathrm{int})=0.0798]$

$99.7 \%$

Semi-empirical from equivalents

0.3201 and 0.1881

Full-matrix least-squares on $\mathrm{F}^{2}$

$5883 / 2 / 412$

1.111

$\mathrm{R} 1=0.0783, \mathrm{wR} 2=0.1967$

$\mathrm{R} 1=0.0848, \mathrm{wR} 2=0.2006$

$0.086(15)$

1.351 and -0.779 e. $\AA^{-3}$ 
Table crystal-3-2. Atomic coordinates ( $\left.\times 10^{4}\right)$ and equivalent isotropic displacement parameters $\left(\AA^{2} \times 10^{3}\right)$ for baran739_0m_a. U(eq) is defined as one third of the trace of the orthogonalized $\mathrm{U}^{\mathrm{ij}}$ tensor.

\begin{tabular}{|c|c|c|c|c|}
\hline & $\mathrm{x}$ & $\mathrm{y}$ & z & $\mathrm{U}(\mathrm{eq})$ \\
\hline $\operatorname{Br}(1)$ & $4590(2)$ & $4623(2)$ & $5791(1)$ & $51(1)$ \\
\hline $\mathrm{S}(1)$ & $-155(3)$ & $1460(2)$ & $2507(2)$ & $31(1)$ \\
\hline $\mathrm{S}(2)$ & $3616(3)$ & $6149(3)$ & $4200(2)$ & $36(1)$ \\
\hline $\mathrm{O}(2)$ & $3260(9)$ & $3305(9)$ & $1679(5)$ & $39(2)$ \\
\hline $\mathrm{O}(3)$ & 2143(9) & $5539(7)$ & $2446(5)$ & $30(2)$ \\
\hline $\mathrm{F}(2)$ & $-392(10)$ & $2243(10)$ & $-50(5)$ & $62(3)$ \\
\hline $\mathrm{O}(7)$ & $2544(9)$ & $2261(7)$ & $3968(5)$ & $33(2)$ \\
\hline $\mathrm{F}(3)$ & $2029(11)$ & 1949(8) & $264(5)$ & $57(2)$ \\
\hline $\mathrm{O}(4)$ & $918(11)$ & $7129(8)$ & $2896(6)$ & $42(2)$ \\
\hline $\mathrm{O}(6)$ & $286(11)$ & $679(8)$ & $3198(6)$ & $44(2)$ \\
\hline $\mathrm{F}(1)$ & $1152(11)$ & $3034(9)$ & $-778(5)$ & $58(2)$ \\
\hline $\mathrm{O}(1)$ & $770(9)$ & $3739(8)$ & $1275(5)$ & $36(2)$ \\
\hline $\mathrm{O}(10)$ & $9029(10)$ & $3986(8)$ & $4063(5)$ & $37(2)$ \\
\hline $\mathrm{O}(9)$ & $6524(10)$ & $5140(8)$ & $3035(5)$ & $40(2)$ \\
\hline $\mathrm{O}(5)$ & $599(10)$ & $1259(8)$ & 1814(6) & $40(2)$ \\
\hline $\mathrm{N}(2)$ & $120(11)$ & $2889(10)$ & $2734(6)$ & $32(2)$ \\
\hline$C(3)$ & 2892(14) & $9346(10)$ & 1294(8) & $36(2)$ \\
\hline$C(26)$ & 6813(13) & $5473(10)$ & $3787(8)$ & $32(3)$ \\
\hline$C(8)$ & $1769(14)$ & $6701(12)$ & $2450(7)$ & $34(3)$ \\
\hline $\mathrm{O}(8)$ & $5349(9)$ & $3637(7)$ & $4092(5)$ & $29(2)$ \\
\hline $\mathrm{N}(1)$ & $5013(17)$ & $9999(10)$ & $-268(9)$ & $56(3)$ \\
\hline $\mathrm{C}(1)$ & $4462(17)$ & $9493(16)$ & $205(8)$ & $51(3)$ \\
\hline$C(7)$ & $4096(13)$ & $7586(11)$ & $843(8)$ & $31(2)$ \\
\hline$C(12)$ & $1975(12)$ & $3447(11)$ & $1842(7)$ & $29(2)$ \\
\hline$C(23)$ & $-2673(17)$ & $2042(15)$ & $1439(10)$ & $54(4)$ \\
\hline $\mathrm{N}(3)$ & $7449(11)$ & $4216(10)$ & 4989(7) & $38(2)$ \\
\hline$C(5)$ & $2512(14)$ & $7434(12)$ & $1889(7)$ & $34(3)$ \\
\hline$C(15)$ & $9954(15)$ & $3342(15)$ & $5427(9)$ & $47(3)$ \\
\hline$C(13)$ & $1644(13)$ & $3423(11)$ & $2698(7)$ & $29(2)$ \\
\hline$C(16)$ & $8829(14)$ & $3845(13)$ & $4766(8)$ & $37(3)$ \\
\hline$C(25)$ & $-2213(13)$ & $1363(13)$ & $2217(9)$ & $42(3)$ \\
\hline
\end{tabular}




\begin{tabular}{lrrrr}
$\mathrm{O}(12)$ & $7488(11)$ & $6416(9)$ & $4015(6)$ & $49(2)$ \\
$\mathrm{C}(21)$ & $3007(12)$ & $2786(10)$ & $3270(7)$ & $28(2)$ \\
$\mathrm{C}(4)$ & $2261(14)$ & $8654(10)$ & $1837(8)$ & $36(2)$ \\
$\mathrm{C}(11)$ & $1094(16)$ & $3917(12)$ & $474(8)$ & $38(3)$ \\
$\mathrm{C}(14)$ & $7095(19)$ & $5910(20)$ & $2452(12)$ & $71(5)$ \\
$\mathrm{C}(22)$ & $-2946(15)$ & $1862(12)$ & $2907(10)$ & $46(4)$ \\
$\mathrm{C}(19)$ & $2827(12)$ & $4697(13)$ & $3813(7)$ & $33(2)$ \\
$\mathrm{C}(9)$ & $1610(12)$ & $4747(10)$ & $3040(6)$ & $27(2)$ \\
$\mathrm{C}(2)$ & $3821(15)$ & $8783(13)$ & $796(8)$ & $38(3)$ \\
$\mathrm{C}(18)$ & $5207(14)$ & $5507(12)$ & $4862(8)$ & $36(3)$ \\
$\mathrm{C}(6)$ & $3453(13)$ & $6883(9)$ & $1399(8)$ & $28(2)$ \\
$\mathrm{C}(10)$ & $996(17)$ & $2775(13)$ & $-8(8)$ & $44(3)$ \\
$\mathrm{C}(24)$ & $-2531(17)$ & $-14(16)$ & $2112(11)$ & $61(4)$ \\
$\mathrm{C}(17)$ & $6172(12)$ & $4661(13)$ & $4423(7)$ & $34(2)$ \\
$\mathrm{C}(20)$ & $3992(12)$ & $3913(11)$ & $3527(7)$ & $28(2)$ \\
& & & & \\
\hline
\end{tabular}


Table crystal-3-3. Bond lengths $[\AA]$ and angles $\left[{ }^{\circ}\right]$ for baran739_0m_a.

\begin{tabular}{|c|c|}
\hline $\operatorname{Br}(1)-C(18)$ & $1.981(14)$ \\
\hline $\mathrm{S}(1)-\mathrm{O}(6)$ & $1.440(10)$ \\
\hline $\mathrm{S}(1)-\mathrm{O}(5)$ & $1.443(9)$ \\
\hline $\mathrm{S}(1)-\mathrm{N}(2)$ & $1.628(11)$ \\
\hline$S(1)-C(25)$ & $1.813(12)$ \\
\hline$S(2)-C(19)$ & $1.821(14)$ \\
\hline$S(2)-C(18)$ & $1.790(14)$ \\
\hline $\mathrm{O}(2)-\mathrm{C}(12)$ & $1.223(14)$ \\
\hline $\mathrm{O}(3)-\mathrm{C}(8)$ & $1.322(15)$ \\
\hline $\mathrm{O}(3)-\mathrm{C}(9)$ & $1.455(13)$ \\
\hline $\mathrm{F}(2)-\mathrm{C}(10)$ & $1.355(18)$ \\
\hline $\mathrm{O}(7)-\mathrm{H}(7)$ & 0.8400 \\
\hline $\mathrm{O}(7)-\mathrm{C}(21)$ & $1.418(14)$ \\
\hline $\mathrm{F}(3)-\mathrm{C}(10)$ & $1.318(16)$ \\
\hline $\mathrm{O}(4)-\mathrm{C}(8)$ & $1.235(14)$ \\
\hline $\mathrm{F}(1)-\mathrm{C}(10)$ & $1.345(16)$ \\
\hline $\mathrm{O}(1)-\mathrm{C}(12)$ & $1.347(14)$ \\
\hline $\mathrm{O}(1)-\mathrm{C}(11)$ & $1.425(15)$ \\
\hline $\mathrm{O}(10)-\mathrm{C}(16)$ & $1.223(15)$ \\
\hline $\mathrm{O}(9)-\mathrm{C}(26)$ & $1.291(16)$ \\
\hline $\mathrm{O}(9)-\mathrm{C}(14)$ & $1.442(19)$ \\
\hline $\mathrm{N}(2)-\mathrm{C}(13)$ & $1.484(14)$ \\
\hline $\mathrm{N}(2)-\mathrm{H}(2)$ & $0.880(3)$ \\
\hline $\mathrm{C}(3)-\mathrm{H}(3)$ & 0.9500 \\
\hline $\mathrm{C}(3)-\mathrm{C}(4)$ & $1.372(17)$ \\
\hline $\mathrm{C}(3)-\mathrm{C}(2)$ & $1.405(17)$ \\
\hline $\mathrm{C}(26)-\mathrm{O}(12)$ & $1.228(15)$ \\
\hline$C(26)-C(17)$ & $1.564(17)$ \\
\hline$C(8)-C(5)$ & $1.470(16)$ \\
\hline $\mathrm{O}(8)-\mathrm{C}(17)$ & $1.407(14)$ \\
\hline $\mathrm{O}(8)-\mathrm{C}(20)$ & $1.435(13)$ \\
\hline $\mathrm{N}(1)-\mathrm{C}(1)$ & $1.138(18)$ \\
\hline$C(1)-C(2)$ & $1.446(18)$ \\
\hline $\mathrm{C}(7)-\mathrm{H}(7 \mathrm{~A})$ & 0.9500 \\
\hline
\end{tabular}




\begin{tabular}{|c|c|}
\hline$C(7)-C(2)$ & $1.341(18)$ \\
\hline$C(7)-C(6)$ & $1.398(17)$ \\
\hline$C(12)-C(13)$ & $1.503(16)$ \\
\hline $\mathrm{C}(23)-\mathrm{H}(23 \mathrm{~A})$ & 0.9800 \\
\hline $\mathrm{C}(23)-\mathrm{H}(23 \mathrm{~B})$ & 0.9800 \\
\hline $\mathrm{C}(23)-\mathrm{H}(23 \mathrm{C})$ & 0.9800 \\
\hline$C(23)-C(25)$ & $1.50(2)$ \\
\hline $\mathrm{N}(3)-\mathrm{H}(3 \mathrm{~A})$ & 0.8800 \\
\hline $\mathrm{N}(3)-\mathrm{C}(16)$ & $1.396(15)$ \\
\hline $\mathrm{N}(3)-\mathrm{C}(17)$ & $1.438(16)$ \\
\hline$C(5)-C(4)$ & $1.362(17)$ \\
\hline$C(5)-C(6)$ & $1.397(15)$ \\
\hline $\mathrm{C}(15)-\mathrm{H}(15 \mathrm{~A})$ & 0.9800 \\
\hline $\mathrm{C}(15)-\mathrm{H}(15 \mathrm{~B})$ & 0.9800 \\
\hline $\mathrm{C}(15)-\mathrm{H}(15 \mathrm{C})$ & 0.9800 \\
\hline$C(15)-C(16)$ & $1.471(19)$ \\
\hline$C(13)-C(21)$ & $1.578(15)$ \\
\hline$C(13)-C(9)$ & $1.568(16)$ \\
\hline$C(25)-C(22)$ & $1.515(18)$ \\
\hline$C(25)-C(24)$ & $1.55(2)$ \\
\hline $\mathrm{C}(21)-\mathrm{H}(21)$ & 1.0000 \\
\hline$C(21)-C(20)$ & $1.538(16)$ \\
\hline $\mathrm{C}(4)-\mathrm{H}(4)$ & 0.9500 \\
\hline $\mathrm{C}(11)-\mathrm{H}(11 \mathrm{~A})$ & 0.9900 \\
\hline $\mathrm{C}(11)-\mathrm{H}(11 \mathrm{~B})$ & 0.9900 \\
\hline$C(11)-C(10)$ & $1.49(2)$ \\
\hline $\mathrm{C}(14)-\mathrm{H}(14 \mathrm{~A})$ & 0.9800 \\
\hline $\mathrm{C}(14)-\mathrm{H}(14 \mathrm{~B})$ & 0.9800 \\
\hline $\mathrm{C}(14)-\mathrm{H}(14 \mathrm{C})$ & 0.9800 \\
\hline $\mathrm{C}(22)-\mathrm{H}(22 \mathrm{~A})$ & 0.9800 \\
\hline $\mathrm{C}(22)-\mathrm{H}(22 \mathrm{~B})$ & 0.9800 \\
\hline $\mathrm{C}(22)-\mathrm{H}(22 \mathrm{C})$ & 0.9800 \\
\hline $\mathrm{C}(19)-\mathrm{H}(19)$ & 1.0000 \\
\hline$C(19)-C(9)$ & $1.542(15)$ \\
\hline$C(19)-C(20)$ & $1.483(16)$ \\
\hline $\mathrm{C}(9)-\mathrm{H}(9)$ & 1.0000 \\
\hline
\end{tabular}




\begin{tabular}{|c|c|}
\hline $\mathrm{C}(18)-\mathrm{H}(18)$ & 1.0000 \\
\hline$C(18)-C(17)$ & $1.528(16)$ \\
\hline $\mathrm{C}(6)-\mathrm{H}(6)$ & 0.9500 \\
\hline $\mathrm{C}(24)-\mathrm{H}(24 \mathrm{~A})$ & 0.9800 \\
\hline $\mathrm{C}(24)-\mathrm{H}(24 \mathrm{~B})$ & 0.9800 \\
\hline $\mathrm{C}(24)-\mathrm{H}(24 \mathrm{C})$ & 0.9800 \\
\hline $\mathrm{C}(20)-\mathrm{H}(20)$ & 1.0000 \\
\hline $\mathrm{O}(6)-\mathrm{S}(1)-\mathrm{O}(5)$ & $116.6(6)$ \\
\hline $\mathrm{O}(6)-\mathrm{S}(1)-\mathrm{N}(2)$ & $112.4(6)$ \\
\hline $\mathrm{O}(6)-\mathrm{S}(1)-\mathrm{C}(25)$ & $108.1(6)$ \\
\hline $\mathrm{O}(5)-\mathrm{S}(1)-\mathrm{N}(2)$ & $105.2(5)$ \\
\hline $\mathrm{O}(5)-\mathrm{S}(1)-\mathrm{C}(25)$ & $110.7(6)$ \\
\hline $\mathrm{N}(2)-\mathrm{S}(1)-\mathrm{C}(25)$ & $103.0(6)$ \\
\hline $\mathrm{C}(18)-\mathrm{S}(2)-\mathrm{C}(19)$ & $95.2(6)$ \\
\hline $\mathrm{C}(8)-\mathrm{O}(3)-\mathrm{C}(9)$ & $117.9(9)$ \\
\hline $\mathrm{C}(21)-\mathrm{O}(7)-\mathrm{H}(7)$ & 109.5 \\
\hline $\mathrm{C}(12)-\mathrm{O}(1)-\mathrm{C}(11)$ & $115.7(9)$ \\
\hline$C(26)-O(9)-C(14)$ & $116.8(13)$ \\
\hline $\mathrm{S}(1)-\mathrm{N}(2)-\mathrm{H}(2)$ & $104(10)$ \\
\hline C(13)-N(2)-S(1) & $118.5(8)$ \\
\hline $\mathrm{C}(13)-\mathrm{N}(2)-\mathrm{H}(2)$ & $106(10)$ \\
\hline $\mathrm{C}(4)-\mathrm{C}(3)-\mathrm{H}(3)$ & 120.5 \\
\hline$C(4)-C(3)-C(2)$ & $119.0(11)$ \\
\hline $\mathrm{C}(2)-\mathrm{C}(3)-\mathrm{H}(3)$ & 120.5 \\
\hline $\mathrm{O}(9)-\mathrm{C}(26)-\mathrm{C}(17)$ & $117.3(10)$ \\
\hline $\mathrm{O}(12)-\mathrm{C}(26)-\mathrm{O}(9)$ & $123.5(12)$ \\
\hline $\mathrm{O}(12)-\mathrm{C}(26)-\mathrm{C}(17)$ & $119.0(11)$ \\
\hline $\mathrm{O}(3)-\mathrm{C}(8)-\mathrm{C}(5)$ & $113.0(10)$ \\
\hline $\mathrm{O}(4)-\mathrm{C}(8)-\mathrm{O}(3)$ & $123.5(11)$ \\
\hline $\mathrm{O}(4)-\mathrm{C}(8)-\mathrm{C}(5)$ & $123.6(12)$ \\
\hline $\mathrm{C}(17)-\mathrm{O}(8)-\mathrm{C}(20)$ & $114.5(9)$ \\
\hline $\mathrm{N}(1)-\mathrm{C}(1)-\mathrm{C}(2)$ & $176.3(16)$ \\
\hline $\mathrm{C}(2)-\mathrm{C}(7)-\mathrm{H}(7 \mathrm{~A})$ & 120.2 \\
\hline$C(2)-C(7)-C(6)$ & $119.5(11)$ \\
\hline $\mathrm{C}(6)-\mathrm{C}(7)-\mathrm{H}(7 \mathrm{~A})$ & 120.2 \\
\hline
\end{tabular}




$\begin{array}{ll}\mathrm{O}(2)-\mathrm{C}(12)-\mathrm{O}(1) & 122.4(11) \\ \mathrm{O}(2)-\mathrm{C}(12)-\mathrm{C}(13) & 122.7(11) \\ \mathrm{O}(1)-\mathrm{C}(12)-\mathrm{C}(13) & 114.6(9) \\ \mathrm{H}(23 \mathrm{~A})-\mathrm{C}(23)-\mathrm{H}(23 \mathrm{~B}) & 109.5 \\ \mathrm{H}(23 \mathrm{~A})-\mathrm{C}(23)-\mathrm{H}(23 \mathrm{C}) & 109.5 \\ \mathrm{H}(23 \mathrm{~B})-\mathrm{C}(23)-\mathrm{H}(23 \mathrm{C}) & 109.5 \\ \mathrm{C}(25)-\mathrm{C}(23)-\mathrm{H}(23 \mathrm{~A}) & 109.5 \\ \mathrm{C}(25)-\mathrm{C}(23)-\mathrm{H}(23 \mathrm{~B}) & 109.5 \\ \mathrm{C}(25)-\mathrm{C}(23)-\mathrm{H}(23 \mathrm{C}) & 109.5 \\ \mathrm{C}(16)-\mathrm{N}(3)-\mathrm{H}(3 \mathrm{~A}) & 109.2 \\ \mathrm{C}(16)-\mathrm{N}(3)-\mathrm{C}(17) & 123.8(11) \\ \mathrm{C}(17)-\mathrm{N}(3)-\mathrm{H}(3 \mathrm{~A}) & 109.3 \\ \mathrm{C}(4)-\mathrm{C}(5)-\mathrm{C}(8) & 119.8(11) \\ \mathrm{C}(4)-\mathrm{C}(5)-\mathrm{C}(6) & 119.9(11) \\ \mathrm{C}(6)-\mathrm{C}(5)-\mathrm{C}(8) & 120.3(11) \\ \mathrm{H}(15 \mathrm{~A})-\mathrm{C}(15)-\mathrm{H}(15 \mathrm{~B}) & 109.5 \\ \mathrm{H}(15 \mathrm{~A})-\mathrm{C}(15)-\mathrm{H}(15 \mathrm{C}) & 109.5 \\ \mathrm{H}(15 \mathrm{~B})-\mathrm{C}(15)-\mathrm{H}(15 \mathrm{C}) & 109.5 \\ \mathrm{C}(16)-\mathrm{C}(15)-\mathrm{H}(15 \mathrm{~A}) & 109.5 \\ \mathrm{C}(16)-\mathrm{C}(15)-\mathrm{H}(15 \mathrm{~B}) & 109.5 \\ \mathrm{C}(16)-\mathrm{C}(15)-\mathrm{H}(15 \mathrm{C}) & 109.5 \\ \mathrm{~N}(2)-\mathrm{C}(13)-\mathrm{C}(12) & 111.4(9) \\ \mathrm{N}(2)-\mathrm{C}(13)-\mathrm{C}(21) & 114.4(10) \\ \mathrm{N}(2)-\mathrm{C}(13)-\mathrm{C}(9) & 106.4(9) \\ \mathrm{C}(12)-\mathrm{C}(13)-\mathrm{C}(21) & 109.5(9) \\ \mathrm{C}(12)-\mathrm{C}(13)-\mathrm{C}(9) & 110.3(9) \\ \mathrm{C}(9)-\mathrm{C}(13)-\mathrm{C}(21) & 104.5(8) \\ \mathrm{O}(10)-\mathrm{C}(16)-\mathrm{N}(3) & 119.2(12) \\ \mathrm{O}(10)-\mathrm{C}(16)-\mathrm{C}(15) & 125.4(12) \\ \mathrm{N}(3)-\mathrm{C}(16)-\mathrm{C}(15) & 115.4(12) \\ \mathrm{C}(23)-\mathrm{C}(25)-\mathrm{S}(1) & 108.9(9) \\ & \end{array}$




\begin{tabular}{|c|c|}
\hline $\mathrm{O}(7)-\mathrm{C}(21)-\mathrm{C}(13)$ & $112.5(9)$ \\
\hline $\mathrm{O}(7)-\mathrm{C}(21)-\mathrm{H}(21)$ & 111.9 \\
\hline $\mathrm{O}(7)-\mathrm{C}(21)-\mathrm{C}(20)$ & 109.1(9) \\
\hline $\mathrm{C}(13)-\mathrm{C}(21)-\mathrm{H}(21)$ & 111.9 \\
\hline$C(20)-C(21)-C(13)$ & $98.9(8)$ \\
\hline $\mathrm{C}(20)-\mathrm{C}(21)-\mathrm{H}(21)$ & 111.9 \\
\hline $\mathrm{C}(3)-\mathrm{C}(4)-\mathrm{H}(4)$ & 119.6 \\
\hline$C(5)-C(4)-C(3)$ & $120.7(11)$ \\
\hline $\mathrm{C}(5)-\mathrm{C}(4)-\mathrm{H}(4)$ & 119.6 \\
\hline $\mathrm{O}(1)-\mathrm{C}(11)-\mathrm{H}(11 \mathrm{~A})$ & 109.0 \\
\hline $\mathrm{O}(1)-\mathrm{C}(11)-\mathrm{H}(11 \mathrm{~B})$ & 109.0 \\
\hline $\mathrm{O}(1)-\mathrm{C}(11)-\mathrm{C}(10)$ & $112.7(11)$ \\
\hline $\mathrm{H}(11 \mathrm{~A})-\mathrm{C}(11)-\mathrm{H}(11 \mathrm{~B})$ & 107.8 \\
\hline $\mathrm{C}(10)-\mathrm{C}(11)-\mathrm{H}(11 \mathrm{~A})$ & 109.0 \\
\hline $\mathrm{C}(10)-\mathrm{C}(11)-\mathrm{H}(11 \mathrm{~B})$ & 109.0 \\
\hline $\mathrm{O}(9)-\mathrm{C}(14)-\mathrm{H}(14 \mathrm{~A})$ & 109.5 \\
\hline $\mathrm{O}(9)-\mathrm{C}(14)-\mathrm{H}(14 \mathrm{~B})$ & 109.5 \\
\hline $\mathrm{O}(9)-\mathrm{C}(14)-\mathrm{H}(14 \mathrm{C})$ & 109.5 \\
\hline $\mathrm{H}(14 \mathrm{~A})-\mathrm{C}(14)-\mathrm{H}(14 \mathrm{~B})$ & 109.5 \\
\hline $\mathrm{H}(14 \mathrm{~A})-\mathrm{C}(14)-\mathrm{H}(14 \mathrm{C})$ & 109.5 \\
\hline $\mathrm{H}(14 \mathrm{~B})-\mathrm{C}(14)-\mathrm{H}(14 \mathrm{C})$ & 109.5 \\
\hline $\mathrm{C}(25)-\mathrm{C}(22)-\mathrm{H}(22 \mathrm{~A})$ & 109.5 \\
\hline $\mathrm{C}(25)-\mathrm{C}(22)-\mathrm{H}(22 \mathrm{~B})$ & 109.5 \\
\hline $\mathrm{C}(25)-\mathrm{C}(22)-\mathrm{H}(22 \mathrm{C})$ & 109.5 \\
\hline $\mathrm{H}(22 \mathrm{~A})-\mathrm{C}(22)-\mathrm{H}(22 \mathrm{~B})$ & 109.5 \\
\hline $\mathrm{H}(22 \mathrm{~A})-\mathrm{C}(22)-\mathrm{H}(22 \mathrm{C})$ & 109.5 \\
\hline $\mathrm{H}(22 \mathrm{~B})-\mathrm{C}(22)-\mathrm{H}(22 \mathrm{C})$ & 109.5 \\
\hline $\mathrm{S}(2)-\mathrm{C}(19)-\mathrm{H}(19)$ & 109.2 \\
\hline $\mathrm{C}(9)-\mathrm{C}(19)-\mathrm{S}(2)$ & 116.2(9) \\
\hline $\mathrm{C}(9)-\mathrm{C}(19)-\mathrm{H}(19)$ & 109.2 \\
\hline $\mathrm{C}(20)-\mathrm{C}(19)-\mathrm{S}(2)$ & $112.2(8)$ \\
\hline $\mathrm{C}(20)-\mathrm{C}(19)-\mathrm{H}(19)$ & 109.2 \\
\hline$C(20)-C(19)-C(9)$ & $100.4(9)$ \\
\hline $\mathrm{O}(3)-\mathrm{C}(9)-\mathrm{C}(13)$ & $106.4(9)$ \\
\hline $\mathrm{O}(3)-\mathrm{C}(9)-\mathrm{C}(19)$ & 109.3(9) \\
\hline $\mathrm{O}(3)-\mathrm{C}(9)-\mathrm{H}(9)$ & 112.6 \\
\hline
\end{tabular}




\begin{tabular}{|c|c|}
\hline $\mathrm{C}(13)-\mathrm{C}(9)-\mathrm{H}(9)$ & 112.6 \\
\hline$C(19)-C(9)-C(13)$ & $102.6(9)$ \\
\hline $\mathrm{C}(19)-\mathrm{C}(9)-\mathrm{H}(9)$ & 112.6 \\
\hline $\mathrm{C}(3)-\mathrm{C}(2)-\mathrm{C}(1)$ & $119.8(13)$ \\
\hline$C(7)-C(2)-C(3)$ & $121.3(11)$ \\
\hline $\mathrm{C}(7)-\mathrm{C}(2)-\mathrm{C}(1)$ & $118.9(12)$ \\
\hline $\mathrm{Br}(1)-\mathrm{C}(18)-\mathrm{H}(18)$ & 107.3 \\
\hline$S(2)-C(18)-\operatorname{Br}(1)$ & $112.7(6)$ \\
\hline $\mathrm{S}(2)-\mathrm{C}(18)-\mathrm{H}(18)$ & 107.3 \\
\hline $\mathrm{C}(17)-\mathrm{C}(18)-\mathrm{Br}(1)$ & $108.7(9)$ \\
\hline $\mathrm{C}(17)-\mathrm{C}(18)-\mathrm{S}(2)$ & $113.1(9)$ \\
\hline $\mathrm{C}(17)-\mathrm{C}(18)-\mathrm{H}(18)$ & 107.3 \\
\hline $\mathrm{C}(7)-\mathrm{C}(6)-\mathrm{H}(6)$ & 120.2 \\
\hline$C(5)-C(6)-C(7)$ & $119.6(10)$ \\
\hline $\mathrm{C}(5)-\mathrm{C}(6)-\mathrm{H}(6)$ & 120.2 \\
\hline $\mathrm{F}(2)-\mathrm{C}(10)-\mathrm{C}(11)$ & $111.6(12)$ \\
\hline $\mathrm{F}(3)-\mathrm{C}(10)-\mathrm{F}(2)$ & $107.1(12)$ \\
\hline $\mathrm{F}(3)-\mathrm{C}(10)-\mathrm{F}(1)$ & $107.6(11)$ \\
\hline $\mathrm{F}(3)-\mathrm{C}(10)-\mathrm{C}(11)$ & $114.9(12)$ \\
\hline $\mathrm{F}(1)-\mathrm{C}(10)-\mathrm{F}(2)$ & $106.0(11)$ \\
\hline $\mathrm{F}(1)-\mathrm{C}(10)-\mathrm{C}(11)$ & $109.2(11)$ \\
\hline $\mathrm{C}(25)-\mathrm{C}(24)-\mathrm{H}(24 \mathrm{~A})$ & 109.5 \\
\hline $\mathrm{C}(25)-\mathrm{C}(24)-\mathrm{H}(24 \mathrm{~B})$ & 109.5 \\
\hline $\mathrm{C}(25)-\mathrm{C}(24)-\mathrm{H}(24 \mathrm{C})$ & 109.5 \\
\hline $\mathrm{H}(24 \mathrm{~A})-\mathrm{C}(24)-\mathrm{H}(24 \mathrm{~B})$ & 109.5 \\
\hline $\mathrm{H}(24 \mathrm{~A})-\mathrm{C}(24)-\mathrm{H}(24 \mathrm{C})$ & 109.5 \\
\hline $\mathrm{H}(24 \mathrm{~B})-\mathrm{C}(24)-\mathrm{H}(24 \mathrm{C})$ & 109.5 \\
\hline $\mathrm{O}(8)-\mathrm{C}(17)-\mathrm{C}(26)$ & $114.5(9)$ \\
\hline $\mathrm{O}(8)-\mathrm{C}(17)-\mathrm{N}(3)$ & $106.7(11)$ \\
\hline $\mathrm{O}(8)-\mathrm{C}(17)-\mathrm{C}(18)$ & $112.5(9)$ \\
\hline $\mathrm{N}(3)-\mathrm{C}(17)-\mathrm{C}(26)$ & $107.8(9)$ \\
\hline $\mathrm{N}(3)-\mathrm{C}(17)-\mathrm{C}(18)$ & $109.7(10)$ \\
\hline $\mathrm{C}(18)-\mathrm{C}(17)-\mathrm{C}(26)$ & $105.6(10)$ \\
\hline $\mathrm{O}(8)-\mathrm{C}(20)-\mathrm{C}(21)$ & $113.0(9)$ \\
\hline $\mathrm{O}(8)-\mathrm{C}(20)-\mathrm{C}(19)$ & $117.7(10)$ \\
\hline $\mathrm{O}(8)-\mathrm{C}(20)-\mathrm{H}(20)$ & 108.7 \\
\hline
\end{tabular}


$\mathrm{C}(21)-\mathrm{C}(20)-\mathrm{H}(20)$

$\mathrm{C}(19)-\mathrm{C}(20)-\mathrm{C}(21)$

$\mathrm{C}(19)-\mathrm{C}(20)-\mathrm{H}(20)$
108.7

99.6(9)

108.7

Symmetry transformations used to generate equivalent atoms: 
Table crystal-3-4. Anisotropic displacement parameters $\left(\AA^{2} \mathrm{x} 10^{3}\right)$ for baran739_0m_a. The anisotropic displacement factor exponent takes the form: $-2 \pi^{2}\left[h^{2} a^{* 2} U^{11}+\ldots+2 h k a^{*} b^{*} U^{12}\right]$

\begin{tabular}{|c|c|c|c|c|c|c|}
\hline & $\mathrm{U}^{11}$ & $U^{22}$ & $\mathrm{U}^{33}$ & $\mathrm{U}^{23}$ & $\mathrm{U}^{13}$ & $\mathrm{U}^{12}$ \\
\hline $\operatorname{Br}(1)$ & $33(1)$ & $79(1)$ & $46(1)$ & $-1(1)$ & $15(1)$ & $6(1)$ \\
\hline $\mathrm{S}(1)$ & $21(1)$ & $26(1)$ & $47(2)$ & $-3(1)$ & $12(1)$ & $0(1)$ \\
\hline$S(2)$ & $28(2)$ & $28(1)$ & $52(2)$ & $-8(1)$ & $9(1)$ & $3(1)$ \\
\hline $\mathrm{O}(2)$ & $22(4)$ & $55(5)$ & $43(5)$ & $-2(4)$ & $13(4)$ & $8(4)$ \\
\hline $\mathrm{O}(3)$ & $25(4)$ & $25(4)$ & $43(5)$ & $0(3)$ & $15(3)$ & $-1(3)$ \\
\hline$F(2)$ & $56(6)$ & $89(7)$ & $42(4)$ & $-5(4)$ & $9(4)$ & $-33(5)$ \\
\hline $\mathrm{O}(7)$ & $29(4)$ & $27(4)$ & $44(5)$ & $6(4)$ & $9(4)$ & $6(3)$ \\
\hline $\mathrm{F}(3)$ & $73(6)$ & $60(6)$ & $41(4)$ & $-5(4)$ & $14(4)$ & $12(4)$ \\
\hline $\mathrm{O}(4)$ & $46(6)$ & $33(5)$ & $53(5)$ & $1(4)$ & $29(4)$ & $9(4)$ \\
\hline $\mathrm{O}(6)$ & $29(5)$ & $38(5)$ & $65(6)$ & $12(4)$ & $12(4)$ & $2(4)$ \\
\hline $\mathrm{F}(1)$ & $67(6)$ & $74(6)$ & $35(4)$ & $5(4)$ & $14(4)$ & $-4(5)$ \\
\hline $\mathrm{O}(1)$ & $24(4)$ & $49(5)$ & $34(4)$ & $6(4)$ & $3(3)$ & $2(4)$ \\
\hline $\mathrm{O}(10)$ & $32(5)$ & $45(5)$ & $35(5)$ & $0(4)$ & $13(4)$ & $5(4)$ \\
\hline $\mathrm{O}(9)$ & $31(5)$ & $57(6)$ & $33(4)$ & $3(4)$ & $9(4)$ & $0(4)$ \\
\hline $\mathrm{O}(5)$ & $38(5)$ & $35(5)$ & $51(5)$ & $-8(4)$ & $22(4)$ & $-3(4)$ \\
\hline $\mathrm{N}(2)$ & $16(5)$ & $47(6)$ & $35(5)$ & $-2(4)$ & $11(4)$ & $-2(4)$ \\
\hline$C(3)$ & $34(4)$ & $19(4)$ & $60(5)$ & $1(4)$ & $23(4)$ & $1(3)$ \\
\hline$C(26)$ & $21(6)$ & $20(5)$ & $57(8)$ & $-2(5)$ & $13(5)$ & $2(4)$ \\
\hline$C(8)$ & $29(6)$ & $36(7)$ & $39(6)$ & $3(5)$ & $16(5)$ & $-4(5)$ \\
\hline $\mathrm{O}(8)$ & $20(4)$ & $20(4)$ & $46(5)$ & $-3(3)$ & $2(3)$ & $4(3)$ \\
\hline $\mathrm{N}(1)$ & $76(9)$ & $30(6)$ & $72(8)$ & $4(5)$ & $42(7)$ & $-9(6)$ \\
\hline$C(1)$ & $53(8)$ & $58(9)$ & $46(7)$ & $9(7)$ & $22(6)$ & $8(8)$ \\
\hline$C(7)$ & $21(6)$ & $32(6)$ & $44(7)$ & $-4(5)$ & $15(5)$ & $0(5)$ \\
\hline$C(12)$ & $18(4)$ & $33(4)$ & $36(4)$ & $-3(3)$ & $5(3)$ & $-5(3)$ \\
\hline$C(23)$ & $31(7)$ & $63(10)$ & $68(10)$ & $-2(8)$ & $5(7)$ & $-19(6)$ \\
\hline $\mathrm{N}(3)$ & $15(4)$ & $53(7)$ & $46(6)$ & $-9(5)$ & $7(4)$ & $2(4)$ \\
\hline$C(5)$ & $25(6)$ & $50(8)$ & $30(6)$ & $6(5)$ & $13(5)$ & $4(5)$ \\
\hline$C(15)$ & $24(6)$ & $65(9)$ & $54(8)$ & $9(7)$ & $11(6)$ & $6(6)$ \\
\hline$C(13)$ & $18(4)$ & $33(4)$ & $36(4)$ & $-3(3)$ & $5(3)$ & $-5(3)$ \\
\hline$C(16)$ & $21(6)$ & $48(7)$ & $43(7)$ & $-8(6)$ & $7(5)$ & $1(5)$ \\
\hline $\mathrm{C}(25)$ & $13(5)$ & $54(8)$ & $61(8)$ & $-10(6)$ & $12(5)$ & $1(5)$ \\
\hline
\end{tabular}




\begin{tabular}{lcccccc}
$\mathrm{O}(12)$ & $47(6)$ & $40(5)$ & $62(6)$ & $-1(5)$ & $19(5)$ & $-7(4)$ \\
$\mathrm{C}(21)$ & $13(5)$ & $25(5)$ & $45(6)$ & $1(5)$ & $6(4)$ & $3(4)$ \\
$\mathrm{C}(4)$ & $34(4)$ & $19(4)$ & $60(5)$ & $1(4)$ & $23(4)$ & $1(3)$ \\
$\mathrm{C}(11)$ & $35(7)$ & $44(7)$ & $36(6)$ & $8(5)$ & $10(5)$ & $1(6)$ \\
$\mathrm{C}(14)$ & $31(8)$ & $108(15)$ & $75(11)$ & $34(10)$ & $8(7)$ & $0(8)$ \\
$\mathrm{C}(22)$ & $23(6)$ & $40(7)$ & $82(11)$ & $-19(7)$ & $24(6)$ & $-3(5)$ \\
$\mathrm{C}(19)$ & $22(5)$ & $38(6)$ & $41(6)$ & $-4(6)$ & $10(4)$ & $2(5)$ \\
$\mathrm{C}(9)$ & $24(5)$ & $21(5)$ & $39(5)$ & $6(5)$ & $12(4)$ & $-4(4)$ \\
$\mathrm{C}(2)$ & $31(7)$ & $49(8)$ & $37(6)$ & $6(6)$ & $14(5)$ & $0(6)$ \\
$\mathrm{C}(18)$ & $21(6)$ & $34(6)$ & $55(8)$ & $-12(6)$ & $9(5)$ & $-6(5)$ \\
$\mathrm{C}(6)$ & $30(6)$ & $11(5)$ & $48(7)$ & $-3(4)$ & $15(5)$ & $3(4)$ \\
$\mathrm{C}(10)$ & $48(8)$ & $44(8)$ & $39(7)$ & $5(6)$ & $9(6)$ & $9(6)$ \\
$\mathrm{C}(24)$ & $29(7)$ & $75(11)$ & $81(11)$ & $-26(9)$ & $15(7)$ & $-11(7)$ \\
$\mathrm{C}(17)$ & $25(5)$ & $37(6)$ & $41(6)$ & $-10(6)$ & $8(4)$ & $-5(6)$ \\
$\mathrm{C}(20)$ & $13(5)$ & $37(6)$ & $35(6)$ & $3(5)$ & $5(4)$ & $2(4)$ \\
& & & & & & \\
\hline
\end{tabular}


Table crystal-3-5. Hydrogen coordinates $\left(\mathrm{x} 10^{4}\right)$ and isotropic displacement parameters $\left(\AA^{2} \mathrm{x} 10^{3}\right)$ for baran739_0m_a.

\begin{tabular}{|c|c|c|c|c|}
\hline & $\mathrm{x}$ & $\mathrm{y}$ & z & $\mathrm{U}(\mathrm{eq})$ \\
\hline $\mathrm{H}(7)$ & 2481 & 1505 & 3910 & 50 \\
\hline $\mathrm{H}(3)$ & 2704 & 10195 & 1254 & 43 \\
\hline $\mathrm{H}(7 \mathrm{~A})$ & 4723 & 7218 & 500 & 38 \\
\hline $\mathrm{H}(23 \mathrm{~A})$ & -2323 & 2885 & 1509 & 81 \\
\hline $\mathrm{H}(23 \mathrm{~B})$ & -3789 & 2027 & 1290 & 81 \\
\hline $\mathrm{H}(23 \mathrm{C})$ & -2210 & 1658 & 1007 & 81 \\
\hline $\mathrm{H}(3 \mathrm{~A})$ & 7637 & 4718 & 5403 & 45 \\
\hline $\mathrm{H}(15 \mathrm{~A})$ & 9471 & 2707 & 5710 & 70 \\
\hline $\mathrm{H}(15 \mathrm{~B})$ & 10330 & 3989 & 5811 & 70 \\
\hline $\mathrm{H}(15 \mathrm{C})$ & 10812 & 2994 & 5201 & 70 \\
\hline $\mathrm{H}(21)$ & 3552 & 2189 & 2968 & 33 \\
\hline $\mathrm{H}(4)$ & 1643 & 9028 & 2181 & 43 \\
\hline $\mathrm{H}(11 \mathrm{~A})$ & 2135 & 4260 & 508 & 46 \\
\hline $\mathrm{H}(11 \mathrm{~B})$ & 363 & 4515 & 189 & 46 \\
\hline $\mathrm{H}(14 \mathrm{~A})$ & 6901 & 5526 & 1916 & 107 \\
\hline $\mathrm{H}(14 \mathrm{~B})$ & 8196 & 6030 & 2617 & 107 \\
\hline $\mathrm{H}(14 \mathrm{C})$ & 6574 & 6697 & 2429 & 107 \\
\hline $\mathrm{H}(22 \mathrm{~A})$ & -2421 & 1533 & 3424 & 70 \\
\hline $\mathrm{H}(22 \mathrm{~B})$ & -4026 & 1626 & 2830 & 70 \\
\hline $\mathrm{H}(22 \mathrm{C})$ & -2867 & 2749 & 2916 & 70 \\
\hline $\mathrm{H}(19)$ & 2393 & 4266 & 4252 & 40 \\
\hline $\mathrm{H}(9)$ & 579 & 4983 & 3157 & 33 \\
\hline $\mathrm{H}(18)$ & 5880 & 6194 & 5090 & 44 \\
\hline $\mathrm{H}(6)$ & 3654 & 6037 & 1443 & 34 \\
\hline $\mathrm{H}(24 \mathrm{~A})$ & -1829 & -368 & 1779 & 91 \\
\hline $\mathrm{H}(24 \mathrm{~B})$ & -3588 & -140 & 1845 & 91 \\
\hline $\mathrm{H}(24 \mathrm{C})$ & -2377 & -405 & 2647 & 91 \\
\hline $\mathrm{H}(20)$ & 4296 & 4288 & 3031 & 34 \\
\hline $\mathrm{H}(2)$ & $-10(160)$ & $2940(140)$ & $3240(30)$ & 50 \\
\hline
\end{tabular}




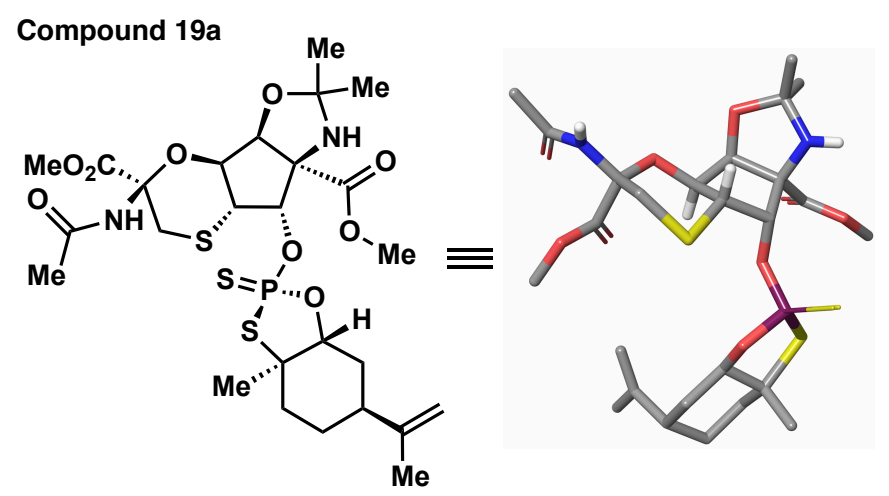

Table crystal-4-1. Crystal data and structure refinement for baran757_0m_a sq.

Identification code

Empirical formula

Formula weight

Temperature

Wavelength

Crystal system

Space group

Unit cell dimensions

Volume

Z, Z'

Density (calculated)

Absorption coefficient

$\mathrm{F}(000)$

Crystal size

Theta range for data collection

Index ranges

Reflections collected

Independent reflections

Completeness to theta $=54.518^{\circ}$

Absorption correction

Max. and min. transmission

Refinement method

Data / restraints / parameters

Goodness-of-fit on $\mathrm{F}^{2}$

Final $\mathrm{R}$ indices [I $>2 \operatorname{sigma}(\mathrm{I})]$

$\mathrm{R}$ indices (all data)

Absolute structure parameter

Extinction coefficient

Largest diff. peak and hole
hc-PV acetone

C26 H39 N2 O9 P S3

650.74

$100.0 \mathrm{~K}$

$1.54178 \AA$

Orthorhombic

$\mathrm{P} 22_{12} 2_{1}$

$\mathrm{a}=6.5797(2) \AA \quad \alpha=90^{\circ}$.

$\mathrm{b}=38.8246(15) \AA \quad \beta=90^{\circ}$.

$\mathrm{c}=41.8141(16) \AA \quad \gamma=90^{\circ}$.

10681.6(7) $\AA^{3}$

12,3

$1.214 \mathrm{Mg} / \mathrm{m}^{3}$

$2.723 \mathrm{~mm}^{-1}$

4128

$0.29 \times 0.07 \times 0.04 \mathrm{~mm}^{3}$

1.553 to $54.517^{\circ}$.

$-6<=\mathrm{h}<=6,-41<=\mathrm{k}<=40,-44<=1<=44$

54241

$13040[\mathrm{R}$ (int) $=0.2390]$

$99.3 \%$

Semi-empirical from equivalents

0.7507 and 0.5408

Full-matrix least-squares on $\mathrm{F}^{2}$

13040 / 0 / 1129

0.994

$\mathrm{R} 1=0.0744, \mathrm{wR} 2=0.1617$

$\mathrm{R} 1=0.1152, \mathrm{wR} 2=0.1768$

$0.06(2)$

$\mathrm{n} / \mathrm{a}$

0.427 and -0.422 e. $\AA^{-3}$ 
Table crystal-4-2. Atomic coordinates ( $\left.\times 10^{4}\right)$ and equivalent isotropic displacement parameters $\left(\AA^{2} \times 10^{3}\right)$ for baran757_0m_a_sq. U(eq) is defined as one third of the trace of the orthogonalized $\mathrm{U}^{\mathrm{ij}}$ tensor.

\begin{tabular}{|c|c|c|c|c|}
\hline & $\mathrm{x}$ & $\mathrm{y}$ & $\mathrm{z}$ & $\mathrm{U}(\mathrm{eq})$ \\
\hline $\mathrm{P}(1)$ & $-3004(5)$ & $4481(1)$ & $4559(1)$ & $40(1)$ \\
\hline $\mathrm{S}(2)$ & $38(5)$ & $4616(1)$ & $4588(1)$ & $45(1)$ \\
\hline $\mathrm{P}(2)$ & 1781(5) & $5830(1)$ & $2988(1)$ & $43(1)$ \\
\hline$S(3)$ & $-6690(5)$ & $3681(1)$ & $4520(1)$ & $49(1)$ \\
\hline$S(4)$ & $4816(4)$ & $5715(1)$ & $2897(1)$ & $47(1)$ \\
\hline $\mathrm{S}(1)$ & $-4975(5)$ & $4821(1)$ & $4441(1)$ & $49(1)$ \\
\hline $\mathrm{S}(9)$ & $3277(6)$ & $9050(1)$ & $3431(1)$ & $58(1)$ \\
\hline$S(5)$ & $-242(5)$ & $5481(1)$ & 2934(1) & $51(1)$ \\
\hline$S(6)$ & $-1870(5)$ & $6498(1)$ & $3367(1)$ & $55(1)$ \\
\hline $\mathrm{S}(8)$ & $10032(6)$ & $9877(1)$ & $3806(1)$ & $63(1)$ \\
\hline $\mathrm{P}(3)$ & 6971(5) & $9784(1)$ & $3725(1)$ & $54(1)$ \\
\hline$S(7)$ & $5107(6)$ & $10164(1)$ & $3729(1)$ & $74(1)$ \\
\hline $\mathrm{O}(23)$ & $5453(12)$ & $9288(2)$ & $2416(2)$ & $45(2)$ \\
\hline $\mathrm{O}(2)$ & $-2918(11)$ & $4149(2)$ & $4332(2)$ & $39(2)$ \\
\hline $\mathrm{O}(12)$ & $3457(12)$ & $5372(2)$ & $3684(2)$ & $48(2)$ \\
\hline $\mathrm{O}(10)$ & $1531(11)$ & $6147(2)$ & $2753(2)$ & $48(2)$ \\
\hline $\mathrm{O}(14)$ & $635(13)$ & $6152(2)$ & $4357(2)$ & $51(2)$ \\
\hline $\mathrm{O}(1)$ & $-3313(12)$ & $4333(2)$ & $4906(2)$ & $47(2)$ \\
\hline $\mathrm{O}(3)$ & $564(13)$ & $4072(2)$ & $3726(2)$ & $48(2)$ \\
\hline $\mathrm{O}(11)$ & $1888(12)$ & $5991(2)$ & $3334(2)$ & $52(2)$ \\
\hline $\mathrm{O}(24)$ & $5193(13)$ & $8737(2)$ & 2793(2) & $46(2)$ \\
\hline $\mathrm{O}(5)$ & $-3860(13)$ & $3456(2)$ & $3522(2)$ & $51(2)$ \\
\hline $\mathrm{O}(20)$ & $7050(13)$ & $9567(2)$ & $3408(2)$ & $54(2)$ \\
\hline $\mathrm{O}(4)$ & $-1475(11)$ & $4529(2)$ & $3773(2)$ & $44(2)$ \\
\hline $\mathrm{O}(6)$ & $-4482(12)$ & $3127(2)$ & $4068(2)$ & $44(2)$ \\
\hline $\mathrm{O}(9)$ & $-2544(15)$ & $3028(2)$ & $4646(2)$ & $54(2)$ \\
\hline $\mathrm{O}(8)$ & $-5429(14)$ & $2823(2)$ & $4868(2)$ & $58(2)$ \\
\hline $\mathrm{O}(21)$ & $8752(14)$ & $10120(2)$ & 2972(3) & $70(3)$ \\
\hline $\mathrm{O}(13)$ & $5324(15)$ & $5795(2)$ & $3900(2)$ & $59(2)$ \\
\hline $\mathrm{O}(15)$ & $222(15)$ & $6750(2)$ & $4017(2)$ & $55(2)$ \\
\hline $\mathrm{N}(3)$ & $398(15)$ & $5627(2)$ & $4109(3)$ & $47(3)$ \\
\hline
\end{tabular}




\begin{tabular}{|c|c|c|c|c|}
\hline $\mathrm{N}(2)$ & $-6562(19)$ & $2685(2)$ & $4237(3)$ & $54(3)$ \\
\hline $\mathrm{O}(19)$ & $6572(15)$ & $9517(2)$ & $3998(2)$ & $65(3)$ \\
\hline $\mathrm{O}(26)$ & $4370(14)$ & $8120(2)$ & $3415(2)$ & $62(3)$ \\
\hline $\mathrm{N}(1)$ & $-4330(14)$ & $4045(2)$ & $3486(3)$ & $43(3)$ \\
\hline $\mathrm{O}(7)$ & $-3757(19)$ & $2372(2)$ & $4313(3)$ & $74(3)$ \\
\hline $\mathrm{O}(17)$ & $-592(14)$ & $7415(2)$ & $3424(3)$ & $65(3)$ \\
\hline $\mathrm{O}(22)$ & $10415(15)$ & $9643(3)$ & 2842(3) & $73(3)$ \\
\hline $\mathrm{O}(25)$ & $7239(17)$ & $8386(2)$ & $3269(3)$ & $66(3)$ \\
\hline $\mathrm{C}(62)$ & $5418(17)$ & $9641(2)$ & 2303(3) & $37(3)$ \\
\hline $\mathrm{C}(61)$ & $6840(20)$ & $9276(2)$ & 2672(3) & $43(3)$ \\
\hline $\mathrm{O}(16)$ & $2264(19)$ & $7116(2)$ & $3549(3)$ & $76(3)$ \\
\hline $\mathrm{C}(41)$ & $3730(20)$ & $5665(3)$ & $3828(3)$ & $40(3)$ \\
\hline $\mathrm{N}(6)$ & $3083(18)$ & $8267(2)$ & $2785(3)$ & $55(3)$ \\
\hline $\mathrm{N}(4)$ & $-1750(30)$ & $7244(3)$ & $4055(3)$ & $82(4)$ \\
\hline $\mathrm{C}(13)$ & $-3069(18)$ & $3986(2)$ & $3777(3)$ & $37(3)$ \\
\hline $\mathrm{C}(4)$ & $-4310(20)$ & 2954(3) & $4634(4)$ & $46(3)$ \\
\hline $\mathrm{N}(5)$ & $5534(15)$ & $9842(2)$ & 2596(3) & $49(3)$ \\
\hline$C(10)$ & $-4126(18)$ & $3733(2)$ & $3289(3)$ & $39(3)$ \\
\hline $\mathrm{C}(14)$ & $-1103(19)$ & 4191(2) & $3763(3)$ & $35(3)$ \\
\hline $\mathrm{C}(9)$ & $-2737(18)$ & $3586(3)$ & $3778(3)$ & $43(3)$ \\
\hline $\mathrm{O}(27)$ & $5937(19)$ & $7955(2)$ & $2707(4)$ & $92(4)$ \\
\hline $\mathrm{C}(3)$ & $-5733(19)$ & $3009(3)$ & $4343(4)$ & $49(4)$ \\
\hline $\mathrm{C}(8)$ & $-3825(19)$ & $3478(3)$ & $4100(3)$ & $43(3)$ \\
\hline$C(60)$ & $5969(19)$ & $9041(2)$ & 2935(3) & $46(3)$ \\
\hline$C(64)$ & $3380(20)$ & $9703(3)$ & $2142(3)$ & $47(3)$ \\
\hline$C(39)$ & $1640(20)$ & $5839(3)$ & $3899(3)$ & $44(3)$ \\
\hline$C(56)$ & $5470(20)$ & $8328(3)$ & $3238(3)$ & $47(3)$ \\
\hline $\mathrm{C}(59)$ & $4417(19)$ & $9262(2)$ & 3094(3) & $46(3)$ \\
\hline$C(20)$ & 1924(19) & $4271(3)$ & $5481(3)$ & $48(3)$ \\
\hline$C(7)$ & $-5403(18)$ & $3735(2)$ & $4137(3)$ & $33(3)$ \\
\hline$C(69)$ & $8400(20)$ & $9343(3)$ & 4095(3) & $51(3)$ \\
\hline$C(65)$ & $6744(19)$ & $9643(3)$ & 2821(3) & $45(3)$ \\
\hline$C(54)$ & $4140(30)$ & $8012(3)$ & $2635(4)$ & $65(5)$ \\
\hline$C(36)$ & $450(20)$ & $5787(3)$ & $4423(3)$ & $48(3)$ \\
\hline$C(33)$ & $-663(18)$ & $6252(3)$ & $3678(4)$ & $46(3)$ \\
\hline $\mathrm{C}(45)$ & $3501(17)$ & $6298(3)$ & $2658(3)$ & $46(3)$ \\
\hline
\end{tabular}




\begin{tabular}{|c|c|c|c|c|}
\hline $\mathrm{C}(23)$ & $-1415(18)$ & 4231(3) & $5054(3)$ & $45(3)$ \\
\hline$C(15)$ & $223(18)$ & $4760(3)$ & $3751(3)$ & $48(3)$ \\
\hline$C(68)$ & $5550(20)$ & $9598(3)$ & $3148(3)$ & $49(3)$ \\
\hline $\mathrm{C}(19)$ & 2073(19) & 4397(3) & $5139(3)$ & $45(3)$ \\
\hline $\mathrm{C}(12)$ & $-6110(20)$ & $3663(3)$ & $3117(3)$ & $55(4)$ \\
\hline $\mathrm{C}(43)$ & $4040(20)$ & $5818(3)$ & 2259(4) & $54(4)$ \\
\hline $\mathrm{C}(66)$ & $8850(20)$ & 9801(3) & $2879(3)$ & $51(4)$ \\
\hline $\mathrm{C}(40)$ & $460(18)$ & $5925(3)$ & $3583(3)$ & $46(3)$ \\
\hline$C(34)$ & $952(17)$ & $6453(2)$ & $3849(3)$ & $40(3)$ \\
\hline $\mathrm{C}(35)$ & $1870(20)$ & 6193(3) & $4078(3)$ & $54(4)$ \\
\hline $\mathrm{C}(18)$ & $73(16)$ & $4523(3)$ & $5022(3)$ & $41(3)$ \\
\hline $\mathrm{C}(46)$ & $3150(20)$ & $6580(3)$ & $2414(3)$ & $54(4)$ \\
\hline $\mathrm{C}(22)$ & $-1700(20)$ & 4111(3) & $5395(3)$ & $58(4)$ \\
\hline $\mathrm{O}(18)$ & $1090(30)$ & $7549(3)$ & 4107(4) & $123(5)$ \\
\hline $\mathrm{C}(63)$ & $7181(19)$ & 9703(3) & 2087(4) & $56(4)$ \\
\hline $\mathrm{C}(47)$ & $5210(20)$ & $6745(3)$ & 2331(4) & $58(4)$ \\
\hline$C(6)$ & $-7474(18)$ & $3249(3)$ & $4413(3)$ & $46(3)$ \\
\hline $\mathrm{C}(29)$ & $-890(20)$ & $6987(3)$ & $3834(4)$ & $57(4)$ \\
\hline $\mathrm{C}(11)$ & $-2290(20)$ & $3750(3)$ & $3070(4)$ & $64(4)$ \\
\hline$C(16)$ & $-4337(16)$ & 4074(3) & $4078(3)$ & $37(3)$ \\
\hline $\mathrm{C}(55)$ & $4040(20)$ & $8516(3)$ & $3002(4)$ & $57(4)$ \\
\hline $\mathrm{C}(30)$ & $490(30)$ & $7180(3)$ & $3590(4)$ & $65(4)$ \\
\hline $\mathrm{C}(70)$ & $9940(20)$ & $9610(4)$ & 4182(4) & $64(4)$ \\
\hline $\mathrm{C}(21)$ & $336(17)$ & $3989(3)$ & $5532(4)$ & $55(4)$ \\
\hline $\mathrm{C}(48)$ & $6730(20)$ & $6462(3)$ & $2245(4)$ & $62(4)$ \\
\hline $\mathrm{C}(17)$ & $-648(17)$ & $4862(3)$ & $5198(3)$ & $42(3)$ \\
\hline $\mathrm{C}(32)$ & $-2700(19)$ & $6823(3)$ & $3653(4)$ & $60(4)$ \\
\hline $\mathrm{C}(42)$ & $5280(20)$ & $5184(3)$ & $3621(3)$ & $47(3)$ \\
\hline $\mathrm{C}(50)$ & $6000(20)$ & 6992(3) & $2584(5)$ & $69(5)$ \\
\hline $\mathrm{C}(2)$ & $-5520(30)$ & 2394(3) & $4214(4)$ & $64(4)$ \\
\hline $\mathrm{C}(73)$ & $11630(30)$ & $9163(4)$ & $4515(4)$ & $81(5)$ \\
\hline $\mathrm{C}(53)$ & $2880(30)$ & $7812(3)$ & $2402(4)$ & $79(5)$ \\
\hline $\mathrm{C}(44)$ & $4774(19)$ & $6014(3)$ & $2543(4)$ & $50(3)$ \\
\hline $\mathrm{C}(58)$ & $2380(20)$ & $8709(3)$ & 3182(4) & $60(4)$ \\
\hline $\mathrm{C}(49)$ & $6895(19)$ & $6175(3)$ & $2495(4)$ & $57(4)$ \\
\hline $\mathrm{C}(57)$ & $5460(30)$ & $7912(4)$ & $3644(4)$ & $77(5)$ \\
\hline
\end{tabular}




\begin{tabular}{lrrrr}
$\mathrm{C}(75)$ & $8000(30)$ & $9088(4)$ & $4370(4)$ & $77(5)$ \\
$\mathrm{C}(38)$ & $-1470(20)$ & $5725(3)$ & $4594(4)$ & $60(4)$ \\
$\mathrm{C}(24)$ & $950(20)$ & $3636(3)$ & $5410(4)$ & $55(4)$ \\
$\mathrm{C}(31)$ & $490(30)$ & $7627(3)$ & $3210(4)$ & $79(5)$ \\
$\mathrm{C}(37)$ & $2220(20)$ & $5670(3)$ & $4615(4)$ & $64(4)$ \\
$\mathrm{C}(28)$ & $-710(40)$ & $7486(4)$ & $4185(5)$ & $88(6)$ \\
$\mathrm{C}(71)$ & $9310(20)$ & $9842(4)$ & $4452(4)$ & $72(4)$ \\
$\mathrm{C}(5)$ & $-4340(20)$ & $2743(4)$ & $5155(4)$ & $69(4)$ \\
$\mathrm{C}(72)$ & $11940(20)$ & $9434(4)$ & $4246(4)$ & $67(4)$ \\
$\mathrm{C}(67)$ & $10660(30)$ & $10301(4)$ & $3027(5)$ & $87(6)$ \\
$\mathrm{C}(52)$ & $5000(30)$ & $7076(3)$ & $2844(5)$ & $79(5)$ \\
$\mathrm{C}(74)$ & $10010(30)$ & $8909(5)$ & $4446(4)$ & $88(5)$ \\
$\mathrm{C}(1)$ & $-6720(30)$ & $2090(3)$ & $4077(4)$ & $77(4)$ \\
$\mathrm{C}(76)$ & $10630(30)$ & $8626(4)$ & $4207(5)$ & $87(6)$ \\
$\mathrm{C}(25)$ & $3010(30)$ & $3505(4)$ & $5528(5)$ & $89(6)$ \\
$\mathrm{C}(26)$ & $-80(30)$ & $3441(3)$ & $5222(4)$ & $68(4)$ \\
$\mathrm{C}(77)$ & $12490(30)$ & $8440(6)$ & $4273(6)$ & $125(9)$ \\
$\mathrm{C}(51)$ & $7990(30)$ & $7150(5)$ & $2520(8)$ & $134(11)$ \\
$\mathrm{C}(78)$ & $9490(30)$ & $8514(5)$ & $3964(5)$ & $104(6)$ \\
$\mathrm{C}(27)$ & $-1820(40)$ & $7715(4)$ & $4412(5)$ & $113(7)$ \\
& & & & \\
\hline
\end{tabular}


Table crystal-4-3. Bond lengths $[\AA]$ and angles $\left[{ }^{\circ}\right]$ for baran757_0m_a_sq.

\begin{tabular}{|c|c|}
\hline $\mathrm{P}(1)-\mathrm{S}(2)$ & $2.073(4)$ \\
\hline $\mathrm{P}(1)-\mathrm{S}(1)$ & $1.917(4)$ \\
\hline $\mathrm{P}(1)-\mathrm{O}(2)$ & $1.603(9)$ \\
\hline $\mathrm{P}(1)-\mathrm{O}(1)$ & $1.570(9)$ \\
\hline$S(2)-C(18)$ & $1.849(13)$ \\
\hline $\mathrm{P}(2)-\mathrm{S}(4)$ & $2.081(4)$ \\
\hline $\mathrm{P}(2)-\mathrm{S}(5)$ & $1.911(4)$ \\
\hline $\mathrm{P}(2)-\mathrm{O}(10)$ & $1.584(9)$ \\
\hline $\mathrm{P}(2)-\mathrm{O}(11)$ & $1.577(10)$ \\
\hline$S(3)-C(7)$ & $1.824(12)$ \\
\hline$S(3)-C(6)$ & $1.809(12)$ \\
\hline$S(4)-C(44)$ & $1.881(14)$ \\
\hline$S(9)-C(59)$ & $1.795(14)$ \\
\hline $\mathrm{S}(9)-\mathrm{C}(58)$ & $1.784(15)$ \\
\hline$S(6)-C(33)$ & $1.798(13)$ \\
\hline$S(6)-C(32)$ & $1.819(16)$ \\
\hline $\mathrm{S}(8)-\mathrm{P}(3)$ & $2.074(5)$ \\
\hline$S(8)-C(70)$ & $1.884(16)$ \\
\hline $\mathrm{P}(3)-\mathrm{S}(7)$ & $1.917(5)$ \\
\hline $\mathrm{P}(3)-\mathrm{O}(20)$ & $1.571(10)$ \\
\hline $\mathrm{P}(3)-\mathrm{O}(19)$ & $1.566(11)$ \\
\hline $\mathrm{O}(23)-\mathrm{C}(62)$ & $1.451(13)$ \\
\hline $\mathrm{O}(23)-\mathrm{C}(61)$ & $1.411(15)$ \\
\hline $\mathrm{O}(2)-\mathrm{C}(16)$ & $1.442(15)$ \\
\hline $\mathrm{O}(12)-\mathrm{C}(41)$ & $1.301(14)$ \\
\hline $\mathrm{O}(12)-\mathrm{C}(42)$ & $1.431(14)$ \\
\hline $\mathrm{O}(10)-\mathrm{C}(45)$ & $1.477(14)$ \\
\hline $\mathrm{O}(14)-\mathrm{C}(36)$ & $1.451(14)$ \\
\hline $\mathrm{O}(14)-\mathrm{C}(35)$ & $1.430(16)$ \\
\hline $\mathrm{O}(1)-\mathrm{C}(23)$ & $1.450(15)$ \\
\hline $\mathrm{O}(3)-\mathrm{C}(14)$ & $1.200(13)$ \\
\hline $\mathrm{O}(11)-\mathrm{C}(40)$ & $1.427(16)$ \\
\hline $\mathrm{O}(24)-\mathrm{C}(60)$ & $1.415(15)$ \\
\hline $\mathrm{O}(24)-\mathrm{C}(55)$ & $1.437(17)$ \\
\hline
\end{tabular}




\begin{tabular}{|c|c|}
\hline $\mathrm{O}(5)-\mathrm{C}(10)$ & $1.461(15)$ \\
\hline $\mathrm{O}(5)-\mathrm{C}(9)$ & $1.394(15)$ \\
\hline $\mathrm{O}(20)-\mathrm{C}(68)$ & $1.471(16)$ \\
\hline $\mathrm{O}(4)-\mathrm{C}(14)$ & $1.336(13)$ \\
\hline $\mathrm{O}(4)-\mathrm{C}(15)$ & $1.435(13)$ \\
\hline $\mathrm{O}(6)-\mathrm{C}(3)$ & $1.486(16)$ \\
\hline $\mathrm{O}(6)-\mathrm{C}(8)$ & $1.437(14)$ \\
\hline $\mathrm{O}(9)-\mathrm{C}(4)$ & $1.198(15)$ \\
\hline $\mathrm{O}(8)-\mathrm{C}(4)$ & $1.325(17)$ \\
\hline $\mathrm{O}(8)-\mathrm{C}(5)$ & $1.433(18)$ \\
\hline $\mathrm{O}(21)-\mathrm{C}(66)$ & $1.300(16)$ \\
\hline $\mathrm{O}(21)-\mathrm{C}(67)$ & $1.457(18)$ \\
\hline $\mathrm{O}(13)-\mathrm{C}(41)$ & $1.204(15)$ \\
\hline $\mathrm{O}(15)-\mathrm{C}(34)$ & $1.433(14)$ \\
\hline $\mathrm{O}(15)-\mathrm{C}(29)$ & $1.399(17)$ \\
\hline $\mathrm{N}(3)-\mathrm{C}(39)$ & $1.453(16)$ \\
\hline $\mathrm{N}(3)-\mathrm{C}(36)$ & $1.452(17)$ \\
\hline $\mathrm{N}(2)-\mathrm{C}(3)$ & $1.443(16)$ \\
\hline $\mathrm{N}(2)-\mathrm{C}(2)$ & $1.326(19)$ \\
\hline $\mathrm{O}(19)-\mathrm{C}(69)$ & $1.437(16)$ \\
\hline $\mathrm{O}(26)-\mathrm{C}(56)$ & $1.314(17)$ \\
\hline $\mathrm{O}(26)-\mathrm{C}(57)$ & $1.441(18)$ \\
\hline $\mathrm{N}(1)-\mathrm{C}(13)$ & $1.492(16)$ \\
\hline $\mathrm{N}(1)-\mathrm{C}(10)$ & $1.472(16)$ \\
\hline $\mathrm{O}(7)-\mathrm{C}(2)$ & $1.233(19)$ \\
\hline $\mathrm{O}(17)-\mathrm{C}(30)$ & $1.349(18)$ \\
\hline $\mathrm{O}(17)-\mathrm{C}(31)$ & $1.407(17)$ \\
\hline $\mathrm{O}(22)-\mathrm{C}(66)$ & $1.208(16)$ \\
\hline $\mathrm{O}(25)-\mathrm{C}(56)$ & $1.191(16)$ \\
\hline $\mathrm{C}(62)-\mathrm{N}(5)$ & $1.455(16)$ \\
\hline $\mathrm{C}(62)-\mathrm{C}(64)$ & $1.520(18)$ \\
\hline$C(62)-C(63)$ & $1.489(18)$ \\
\hline $\mathrm{C}(61)-\mathrm{C}(60)$ & $1.536(18)$ \\
\hline$C(61)-C(65)$ & $1.557(16)$ \\
\hline $\mathrm{O}(16)-\mathrm{C}(30)$ & $1.204(18)$ \\
\hline $\mathrm{C}(41)-\mathrm{C}(39)$ & $1.557(18)$ \\
\hline
\end{tabular}




\begin{tabular}{|c|c|}
\hline $\mathrm{N}(6)-\mathrm{C}(54)$ & $1.36(2)$ \\
\hline $\mathrm{N}(6)-\mathrm{C}(55)$ & $1.469(18)$ \\
\hline $\mathrm{N}(4)-\mathrm{C}(29)$ & $1.474(19)$ \\
\hline $\mathrm{N}(4)-\mathrm{C}(28)$ & $1.28(2)$ \\
\hline$C(13)-C(14)$ & $1.520(16)$ \\
\hline$C(13)-C(9)$ & $1.568(15)$ \\
\hline$C(13)-C(16)$ & $1.548(18)$ \\
\hline $\mathrm{C}(4)-\mathrm{C}(3)$ & $1.55(2)$ \\
\hline $\mathrm{N}(5)-\mathrm{C}(65)$ & $1.458(16)$ \\
\hline $\mathrm{C}(10)-\mathrm{C}(12)$ & $1.516(18)$ \\
\hline$C(10)-C(11)$ & $1.513(19)$ \\
\hline $\mathrm{C}(9)-\mathrm{C}(8)$ & $1.579(19)$ \\
\hline $\mathrm{O}(27)-\mathrm{C}(54)$ & $1.240(17)$ \\
\hline $\mathrm{C}(3)-\mathrm{C}(6)$ & $1.506(18)$ \\
\hline $\mathrm{C}(8)-\mathrm{C}(7)$ & $1.447(16)$ \\
\hline $\mathrm{C}(60)-\mathrm{C}(59)$ & $1.490(18)$ \\
\hline$C(39)-C(40)$ & $1.566(19)$ \\
\hline$C(39)-C(35)$ & $1.574(18)$ \\
\hline$C(56)-C(55)$ & $1.55(2)$ \\
\hline $\mathrm{C}(59)-\mathrm{C}(68)$ & $1.522(17)$ \\
\hline$C(20)-C(19)$ & $1.513(19)$ \\
\hline$C(20)-C(21)$ & $1.528(18)$ \\
\hline$C(7)-C(16)$ & $1.512(15)$ \\
\hline $\mathrm{C}(69)-\mathrm{C}(70)$ & $1.49(2)$ \\
\hline $\mathrm{C}(69)-\mathrm{C}(75)$ & $1.54(2)$ \\
\hline $\mathrm{C}(65)-\mathrm{C}(68)$ & $1.58(2)$ \\
\hline $\mathrm{C}(65)-\mathrm{C}(66)$ & $1.532(19)$ \\
\hline $\mathrm{C}(54)-\mathrm{C}(53)$ & $1.50(2)$ \\
\hline$C(36)-C(38)$ & $1.474(19)$ \\
\hline$C(36)-C(37)$ & $1.49(2)$ \\
\hline$C(33)-C(40)$ & $1.524(17)$ \\
\hline$C(33)-C(34)$ & $1.500(17)$ \\
\hline$C(45)-C(46)$ & $1.515(18)$ \\
\hline$C(45)-C(44)$ & $1.464(17)$ \\
\hline$C(23)-C(18)$ & $1.506(16)$ \\
\hline$C(23)-C(22)$ & $1.51(2)$ \\
\hline
\end{tabular}




\begin{tabular}{|c|c|}
\hline$C(19)-C(18)$ & $1.488(18)$ \\
\hline C(43)-C(44) & $1.49(2)$ \\
\hline$C(34)-C(35)$ & $1.520(19)$ \\
\hline$C(18)-C(17)$ & $1.582(16)$ \\
\hline$C(46)-C(47)$ & $1.54(2)$ \\
\hline$C(22)-C(21)$ & $1.53(2)$ \\
\hline $\mathrm{O}(18)-\mathrm{C}(28)$ & $1.25(2)$ \\
\hline $\mathrm{C}(47)-\mathrm{C}(48)$ & $1.53(2)$ \\
\hline$C(47)-C(50)$ & $1.52(2)$ \\
\hline$C(29)-C(30)$ & $1.56(2)$ \\
\hline$C(29)-C(32)$ & $1.55(2)$ \\
\hline $\mathrm{C}(55)-\mathrm{C}(58)$ & $1.53(2)$ \\
\hline$C(70)-C(71)$ & $1.50(2)$ \\
\hline $\mathrm{C}(70)-\mathrm{C}(72)$ & $1.51(2)$ \\
\hline$C(21)-C(24)$ & $1.516(19)$ \\
\hline$C(48)-C(49)$ & $1.53(2)$ \\
\hline$C(50)-C(52)$ & $1.31(2)$ \\
\hline$C(50)-C(51)$ & $1.47(2)$ \\
\hline$C(2)-C(1)$ & $1.53(2)$ \\
\hline$C(73)-C(72)$ & $1.55(2)$ \\
\hline$C(73)-C(74)$ & $1.48(3)$ \\
\hline $\mathrm{C}(44)-\mathrm{C}(49)$ & $1.543(18)$ \\
\hline$C(75)-C(74)$ & $1.53(2)$ \\
\hline$C(24)-C(25)$ & $1.53(2)$ \\
\hline$C(24)-C(26)$ & $1.29(2)$ \\
\hline$C(28)-C(27)$ & $1.49(3)$ \\
\hline$C(74)-C(76)$ & $1.54(3)$ \\
\hline$C(76)-C(77)$ & $1.45(3)$ \\
\hline$C(76)-C(78)$ & $1.34(3)$ \\
\hline $\mathrm{S}(1)-\mathrm{P}(1)-\mathrm{S}(2)$ & $119.58(18)$ \\
\hline $\mathrm{O}(2)-\mathrm{P}(1)-\mathrm{S}(2)$ & $101.8(3)$ \\
\hline $\mathrm{O}(2)-\mathrm{P}(1)-\mathrm{S}(1)$ & $115.2(3)$ \\
\hline $\mathrm{O}(1)-\mathrm{P}(1)-\mathrm{S}(2)$ & $99.5(4)$ \\
\hline $\mathrm{O}(1)-\mathrm{P}(1)-\mathrm{S}(1)$ & $113.7(3)$ \\
\hline $\mathrm{O}(1)-\mathrm{P}(1)-\mathrm{O}(2)$ & $104.9(4)$ \\
\hline
\end{tabular}




\begin{tabular}{|c|c|}
\hline $\mathrm{C}(18)-\mathrm{S}(2)-\mathrm{P}(1)$ & $91.1(4)$ \\
\hline $\mathrm{S}(5)-\mathrm{P}(2)-\mathrm{S}(4)$ & $119.68(18)$ \\
\hline $\mathrm{O}(10)-\mathrm{P}(2)-\mathrm{S}(4)$ & $98.8(3)$ \\
\hline $\mathrm{O}(10)-\mathrm{P}(2)-\mathrm{S}(5)$ & $113.9(3)$ \\
\hline $\mathrm{O}(11)-\mathrm{P}(2)-\mathrm{S}(4)$ & $102.1(4)$ \\
\hline $\mathrm{O}(11)-\mathrm{P}(2)-\mathrm{S}(5)$ & $114.9(4)$ \\
\hline $\mathrm{O}(11)-\mathrm{P}(2)-\mathrm{O}(10)$ & $105.3(4)$ \\
\hline$C(6)-S(3)-C(7)$ & $91.4(5)$ \\
\hline C(44)-S(4)-P(2) & $89.8(4)$ \\
\hline $\mathrm{C}(58)-\mathrm{S}(9)-\mathrm{C}(59)$ & $91.3(6)$ \\
\hline $\mathrm{C}(33)-\mathrm{S}(6)-\mathrm{C}(32)$ & $91.5(6)$ \\
\hline $\mathrm{C}(70)-\mathrm{S}(8)-\mathrm{P}(3)$ & $90.5(4)$ \\
\hline $\mathrm{S}(7)-\mathrm{P}(3)-\mathrm{S}(8)$ & $119.1(2)$ \\
\hline $\mathrm{O}(20)-\mathrm{P}(3)-\mathrm{S}(8)$ & $101.5(4)$ \\
\hline $\mathrm{O}(20)-\mathrm{P}(3)-\mathrm{S}(7)$ & $116.3(4)$ \\
\hline $\mathrm{O}(19)-\mathrm{P}(3)-\mathrm{S}(8)$ & 99.1(4) \\
\hline $\mathrm{O}(19)-\mathrm{P}(3)-\mathrm{S}(7)$ & $113.3(4)$ \\
\hline $\mathrm{O}(19)-\mathrm{P}(3)-\mathrm{O}(20)$ & $105.2(5)$ \\
\hline $\mathrm{C}(61)-\mathrm{O}(23)-\mathrm{C}(62)$ & $106.8(8)$ \\
\hline $\mathrm{C}(16)-\mathrm{O}(2)-\mathrm{P}(1)$ & $125.1(6)$ \\
\hline $\mathrm{C}(41)-\mathrm{O}(12)-\mathrm{C}(42)$ & $114.7(10)$ \\
\hline $\mathrm{C}(45)-\mathrm{O}(10)-\mathrm{P}(2)$ & $112.6(7)$ \\
\hline $\mathrm{C}(35)-\mathrm{O}(14)-\mathrm{C}(36)$ & $108.0(9)$ \\
\hline $\mathrm{C}(23)-\mathrm{O}(1)-\mathrm{P}(1)$ & $112.5(7)$ \\
\hline $\mathrm{C}(40)-\mathrm{O}(11)-\mathrm{P}(2)$ & $124.6(7)$ \\
\hline $\mathrm{C}(60)-\mathrm{O}(24)-\mathrm{C}(55)$ & $115.8(10)$ \\
\hline $\mathrm{C}(9)-\mathrm{O}(5)-\mathrm{C}(10)$ & $108.1(8)$ \\
\hline $\mathrm{C}(68)-\mathrm{O}(20)-\mathrm{P}(3)$ & $123.7(7)$ \\
\hline $\mathrm{C}(14)-\mathrm{O}(4)-\mathrm{C}(15)$ & $118.0(9)$ \\
\hline $\mathrm{C}(8)-\mathrm{O}(6)-\mathrm{C}(3)$ & $112.9(9)$ \\
\hline $\mathrm{C}(4)-\mathrm{O}(8)-\mathrm{C}(5)$ & $115.0(11)$ \\
\hline $\mathrm{C}(66)-\mathrm{O}(21)-\mathrm{C}(67)$ & $117.8(12)$ \\
\hline $\mathrm{C}(29)-\mathrm{O}(15)-\mathrm{C}(34)$ & $115.8(10)$ \\
\hline $\mathrm{C}(36)-\mathrm{N}(3)-\mathrm{C}(39)$ & $107.0(9)$ \\
\hline $\mathrm{C}(2)-\mathrm{N}(2)-\mathrm{C}(3)$ & $124.7(13)$ \\
\hline C(69)-O(19)-P(3) & $112.2(8)$ \\
\hline
\end{tabular}




\begin{tabular}{|c|c|}
\hline $\mathrm{C}(56)-\mathrm{O}(26)-\mathrm{C}(57)$ & $116.3(12)$ \\
\hline $\mathrm{C}(10)-\mathrm{N}(1)-\mathrm{C}(13)$ & $106.3(8)$ \\
\hline $\mathrm{C}(30)-\mathrm{O}(17)-\mathrm{C}(31)$ & $117.0(12)$ \\
\hline $\mathrm{O}(23)-\mathrm{C}(62)-\mathrm{N}(5)$ & $103.4(9)$ \\
\hline $\mathrm{O}(23)-\mathrm{C}(62)-\mathrm{C}(64)$ & $107.9(8)$ \\
\hline $\mathrm{O}(23)-\mathrm{C}(62)-\mathrm{C}(63)$ & $109.8(8)$ \\
\hline $\mathrm{N}(5)-\mathrm{C}(62)-\mathrm{C}(64)$ & $109.5(9)$ \\
\hline $\mathrm{N}(5)-\mathrm{C}(62)-\mathrm{C}(63)$ & $112.5(10)$ \\
\hline$C(63)-C(62)-C(64)$ & $113.2(11)$ \\
\hline $\mathrm{O}(23)-\mathrm{C}(61)-\mathrm{C}(60)$ & $108.8(10)$ \\
\hline $\mathrm{O}(23)-\mathrm{C}(61)-\mathrm{C}(65)$ & $104.3(8)$ \\
\hline$C(60)-C(61)-C(65)$ & $104.0(10)$ \\
\hline $\mathrm{O}(12)-\mathrm{C}(41)-\mathrm{C}(39)$ & $110.3(10)$ \\
\hline $\mathrm{O}(13)-\mathrm{C}(41)-\mathrm{O}(12)$ & $126.9(11)$ \\
\hline $\mathrm{O}(13)-\mathrm{C}(41)-\mathrm{C}(39)$ & $122.8(10)$ \\
\hline $\mathrm{C}(54)-\mathrm{N}(6)-\mathrm{C}(55)$ & $122.9(12)$ \\
\hline $\mathrm{C}(28)-\mathrm{N}(4)-\mathrm{C}(29)$ & $123.8(17)$ \\
\hline $\mathrm{N}(1)-\mathrm{C}(13)-\mathrm{C}(14)$ & 111.1(9) \\
\hline $\mathrm{N}(1)-\mathrm{C}(13)-\mathrm{C}(9)$ & $103.3(9)$ \\
\hline $\mathrm{N}(1)-\mathrm{C}(13)-\mathrm{C}(16)$ & 109.3(9) \\
\hline $\mathrm{C}(14)-\mathrm{C}(13)-\mathrm{C}(9)$ & $113.6(10)$ \\
\hline$C(14)-C(13)-C(16)$ & 112.1(9) \\
\hline$C(16)-C(13)-C(9)$ & 107.0(9) \\
\hline $\mathrm{O}(9)-\mathrm{C}(4)-\mathrm{O}(8)$ & $126.9(14)$ \\
\hline $\mathrm{O}(9)-\mathrm{C}(4)-\mathrm{C}(3)$ & $125.8(13)$ \\
\hline $\mathrm{O}(8)-\mathrm{C}(4)-\mathrm{C}(3)$ & $107.2(11)$ \\
\hline $\mathrm{C}(62)-\mathrm{N}(5)-\mathrm{C}(65)$ & $106.8(8)$ \\
\hline $\mathrm{O}(5)-\mathrm{C}(10)-\mathrm{N}(1)$ & $104.0(9)$ \\
\hline $\mathrm{O}(5)-\mathrm{C}(10)-\mathrm{C}(12)$ & $106.8(9)$ \\
\hline $\mathrm{O}(5)-\mathrm{C}(10)-\mathrm{C}(11)$ & $110.0(9)$ \\
\hline $\mathrm{N}(1)-\mathrm{C}(10)-\mathrm{C}(12)$ & $109.4(9)$ \\
\hline $\mathrm{N}(1)-\mathrm{C}(10)-\mathrm{C}(11)$ & $111.9(9)$ \\
\hline$C(11)-C(10)-C(12)$ & 114.1(11) \\
\hline $\mathrm{O}(3)-\mathrm{C}(14)-\mathrm{O}(4)$ & $123.4(10)$ \\
\hline $\mathrm{O}(3)-\mathrm{C}(14)-\mathrm{C}(13)$ & $125.5(9)$ \\
\hline $\mathrm{O}(4)-\mathrm{C}(14)-\mathrm{C}(13)$ & $110.9(10)$ \\
\hline
\end{tabular}




\begin{tabular}{|c|c|}
\hline $\mathrm{O}(5)-\mathrm{C}(9)-\mathrm{C}(13)$ & $106.4(9)$ \\
\hline $\mathrm{O}(5)-\mathrm{C}(9)-\mathrm{C}(8)$ & $108.5(9)$ \\
\hline $\mathrm{C}(13)-\mathrm{C}(9)-\mathrm{C}(8)$ & $101.6(9)$ \\
\hline $\mathrm{O}(6)-\mathrm{C}(3)-\mathrm{C}(4)$ & $108.3(10)$ \\
\hline $\mathrm{O}(6)-\mathrm{C}(3)-\mathrm{C}(6)$ & $112.4(9)$ \\
\hline $\mathrm{N}(2)-\mathrm{C}(3)-\mathrm{O}(6)$ & $103.9(11)$ \\
\hline $\mathrm{N}(2)-\mathrm{C}(3)-\mathrm{C}(4)$ & $110.5(9)$ \\
\hline $\mathrm{N}(2)-\mathrm{C}(3)-\mathrm{C}(6)$ & $108.2(10)$ \\
\hline$C(6)-C(3)-C(4)$ & $113.1(12)$ \\
\hline $\mathrm{O}(6)-\mathrm{C}(8)-\mathrm{C}(9)$ & $108.1(10)$ \\
\hline $\mathrm{O}(6)-\mathrm{C}(8)-\mathrm{C}(7)$ & $116.5(10)$ \\
\hline$C(7)-C(8)-C(9)$ & 103.6(9) \\
\hline $\mathrm{O}(24)-\mathrm{C}(60)-\mathrm{C}(61)$ & $109.3(11)$ \\
\hline $\mathrm{O}(24)-\mathrm{C}(60)-\mathrm{C}(59)$ & $114.8(10)$ \\
\hline$C(59)-C(60)-C(61)$ & $103.6(8)$ \\
\hline $\mathrm{N}(3)-\mathrm{C}(39)-\mathrm{C}(41)$ & 111.4(9) \\
\hline $\mathrm{N}(3)-\mathrm{C}(39)-\mathrm{C}(40)$ & $110.5(10)$ \\
\hline $\mathrm{N}(3)-\mathrm{C}(39)-\mathrm{C}(35)$ & $105.0(10)$ \\
\hline $\mathrm{C}(41)-\mathrm{C}(39)-\mathrm{C}(40)$ & $111.7(10)$ \\
\hline $\mathrm{C}(41)-\mathrm{C}(39)-\mathrm{C}(35)$ & $112.4(11)$ \\
\hline $\mathrm{C}(40)-\mathrm{C}(39)-\mathrm{C}(35)$ & $105.4(9)$ \\
\hline $\mathrm{O}(26)-\mathrm{C}(56)-\mathrm{C}(55)$ & $108.4(12)$ \\
\hline $\mathrm{O}(25)-\mathrm{C}(56)-\mathrm{O}(26)$ & $126.4(13)$ \\
\hline $\mathrm{O}(25)-\mathrm{C}(56)-\mathrm{C}(55)$ & $124.9(12)$ \\
\hline $\mathrm{C}(60)-\mathrm{C}(59)-\mathrm{S}(9)$ & $111.9(8)$ \\
\hline $\mathrm{C}(60)-\mathrm{C}(59)-\mathrm{C}(68)$ & $102.9(10)$ \\
\hline $\mathrm{C}(68)-\mathrm{C}(59)-\mathrm{S}(9)$ & $118.9(10)$ \\
\hline$C(19)-C(20)-C(21)$ & 114.1(11) \\
\hline $\mathrm{C}(8)-\mathrm{C}(7)-\mathrm{S}(3)$ & $110.4(8)$ \\
\hline$C(8)-C(7)-C(16)$ & 104.4(9) \\
\hline$C(16)-C(7)-S(3)$ & $117.3(8)$ \\
\hline $\mathrm{O}(19)-\mathrm{C}(69)-\mathrm{C}(70)$ & $108.1(10)$ \\
\hline $\mathrm{O}(19)-\mathrm{C}(69)-\mathrm{C}(75)$ & $111.6(12)$ \\
\hline $\mathrm{C}(70)-\mathrm{C}(69)-\mathrm{C}(75)$ & $112.3(12)$ \\
\hline $\mathrm{C}(61)-\mathrm{C}(65)-\mathrm{C}(68)$ & 105.4(9) \\
\hline $\mathrm{N}(5)-\mathrm{C}(65)-\mathrm{C}(61)$ & $104.5(10)$ \\
\hline
\end{tabular}




$\begin{array}{ll}\mathrm{N}(5)-\mathrm{C}(65)-\mathrm{C}(68) & 110.3(10) \\ \mathrm{N}(5)-\mathrm{C}(65)-\mathrm{C}(66) & 112.5(9) \\ \mathrm{C}(66)-\mathrm{C}(65)-\mathrm{C}(61) & 113.0(11) \\ \mathrm{C}(66)-\mathrm{C}(65)-\mathrm{C}(68) & 110.8(11) \\ \mathrm{N}(6)-\mathrm{C}(54)-\mathrm{C}(53) & 113.1(14) \\ \mathrm{O}(27)-\mathrm{C}(54)-\mathrm{N}(6) & 120.3(14) \\ \mathrm{O}(27)-\mathrm{C}(54)-\mathrm{C}(53) & 126.6(15) \\ \mathrm{O}(14)-\mathrm{C}(36)-\mathrm{N}(3) & 104.3(10) \\ \mathrm{O}(14)-\mathrm{C}(36)-\mathrm{C}(38) & 108.8(10) \\ \mathrm{O}(14)-\mathrm{C}(36)-\mathrm{C}(37) & 109.6(10) \\ \mathrm{N}(3)-\mathrm{C}(36)-\mathrm{C}(38) & 110.6(11) \\ \mathrm{N}(3)-\mathrm{C}(36)-\mathrm{C}(37) & 112.1(11) \\ \mathrm{C}(38)-\mathrm{C}(36)-\mathrm{C}(37) & 111.1(11) \\ \mathrm{C}(40)-\mathrm{C}(33)-\mathrm{S}(6) & 118.0(10) \\ \mathrm{C}(34)-\mathrm{C}(33)-\mathrm{S}(6) & 112.4(7) \\ \mathrm{C}(34)-\mathrm{C}(33)-\mathrm{C}(40) & 102.4(9) \\ \mathrm{O}(10)-\mathrm{C}(45)-\mathrm{C}(46) & 109.5(10) \\ \mathrm{C}(44)-\mathrm{C}(45)-\mathrm{O}(10) & 107.0(9) \\ \mathrm{C}(44)-\mathrm{C}(45)-\mathrm{C}(46) & 114.2(12) \\ \mathrm{O}(1)-\mathrm{C}(23)-\mathrm{C}(18) & 108.3(9) \\ \mathrm{O}(1)-\mathrm{C}(23)-\mathrm{C}(22) & 112.3(10) \\ \mathrm{C}(18)-\mathrm{C}(23)-\mathrm{C}(22) & 113.4(11) \\ \mathrm{O}(20)-\mathrm{C}(68)-\mathrm{C}(59) & 111.6(10) \\ \mathrm{O}(20)-\mathrm{C}(68)-\mathrm{C}(65) & 108.4(10) \\ \mathrm{C}(59)-\mathrm{C}(68)-\mathrm{C}(65) & 102.0(10) \\ \mathrm{C}(18)-\mathrm{C}(19)-\mathrm{C}(20) & 111.2(10) \\ \mathrm{O}(21)-\mathrm{C}(66)-\mathrm{C}(65) & 112.6(12) \\ \mathrm{O}(22)-\mathrm{C}(66)-\mathrm{O}(21) & 124.1(12) \\ \mathrm{O}(22)-\mathrm{C}(66)-\mathrm{C}(65) & 123.3(11) \\ \mathrm{O}(11)-\mathrm{C}(40)-\mathrm{C}(39) & 109.1(9) \\ \mathrm{O}(11)-\mathrm{C}(40)-\mathrm{C}(33) & 111.19) \\ \mathrm{C}(33)-\mathrm{C}(40)-\mathrm{C}(39) & \mathrm{C}(15)-\mathrm{C}(34)-\mathrm{C}(33) \\ & \end{array}$




\begin{tabular}{|c|c|}
\hline $\mathrm{O}(14)-\mathrm{C}(35)-\mathrm{C}(34)$ & $111.0(11)$ \\
\hline$C(34)-C(35)-C(39)$ & $103.9(11)$ \\
\hline $\mathrm{C}(23)-\mathrm{C}(18)-\mathrm{S}(2)$ & $103.1(8)$ \\
\hline$C(23)-C(18)-C(17)$ & $113.0(9)$ \\
\hline $\mathrm{C}(19)-\mathrm{C}(18)-\mathrm{S}(2)$ & $113.6(9)$ \\
\hline$C(19)-C(18)-C(23)$ & $107.3(9)$ \\
\hline$C(19)-C(18)-C(17)$ & $112.7(10)$ \\
\hline $\mathrm{C}(17)-\mathrm{C}(18)-\mathrm{S}(2)$ & $106.9(8)$ \\
\hline$C(45)-C(46)-C(47)$ & $108.6(11)$ \\
\hline $\mathrm{C}(23)-\mathrm{C}(22)-\mathrm{C}(21)$ & $109.7(12)$ \\
\hline$C(48)-C(47)-C(46)$ & $109.3(9)$ \\
\hline$C(50)-C(47)-C(46)$ & $114.2(13)$ \\
\hline$C(50)-C(47)-C(48)$ & $113.0(13)$ \\
\hline $\mathrm{C}(3)-\mathrm{C}(6)-\mathrm{S}(3)$ & $113.8(8)$ \\
\hline $\mathrm{O}(15)-\mathrm{C}(29)-\mathrm{N}(4)$ & $107.6(12)$ \\
\hline $\mathrm{O}(15)-\mathrm{C}(29)-\mathrm{C}(30)$ & $111.8(12)$ \\
\hline $\mathrm{O}(15)-\mathrm{C}(29)-\mathrm{C}(32)$ & $113.6(9)$ \\
\hline N(4)-C(29)-C(30) & $108.0(10)$ \\
\hline $\mathrm{N}(4)-\mathrm{C}(29)-\mathrm{C}(32)$ & $106.7(12)$ \\
\hline $\mathrm{C}(32)-\mathrm{C}(29)-\mathrm{C}(30)$ & $108.9(13)$ \\
\hline $\mathrm{O}(2)-\mathrm{C}(16)-\mathrm{C}(13)$ & $107.1(8)$ \\
\hline $\mathrm{O}(2)-\mathrm{C}(16)-\mathrm{C}(7)$ & $110.9(10)$ \\
\hline $\mathrm{C}(7)-\mathrm{C}(16)-\mathrm{C}(13)$ & $100.9(8)$ \\
\hline $\mathrm{O}(24)-\mathrm{C}(55)-\mathrm{N}(6)$ & $104.3(11)$ \\
\hline $\mathrm{O}(24)-\mathrm{C}(55)-\mathrm{C}(56)$ & $110.5(11)$ \\
\hline $\mathrm{O}(24)-\mathrm{C}(55)-\mathrm{C}(58)$ & $112.6(9)$ \\
\hline $\mathrm{N}(6)-\mathrm{C}(55)-\mathrm{C}(56)$ & $110.1(9)$ \\
\hline $\mathrm{N}(6)-\mathrm{C}(55)-\mathrm{C}(58)$ & $108.6(11)$ \\
\hline $\mathrm{C}(58)-\mathrm{C}(55)-\mathrm{C}(56)$ & $110.6(13)$ \\
\hline $\mathrm{O}(17)-\mathrm{C}(30)-\mathrm{C}(29)$ & $110.7(14)$ \\
\hline $\mathrm{O}(16)-\mathrm{C}(30)-\mathrm{O}(17)$ & $125.4(15)$ \\
\hline $\mathrm{O}(16)-\mathrm{C}(30)-\mathrm{C}(29)$ & $123.8(14)$ \\
\hline C(69)-C(70)-S(8) & $101.6(10)$ \\
\hline $\mathrm{C}(69)-\mathrm{C}(70)-\mathrm{C}(71)$ & $114.5(12)$ \\
\hline$C(69)-C(70)-C(72)$ & $108.8(12)$ \\
\hline $\mathrm{C}(71)-\mathrm{C}(70)-\mathrm{S}(8)$ & $107.8(11)$ \\
\hline
\end{tabular}




\begin{tabular}{|c|c|}
\hline$C(71)-C(70)-C(72)$ & $112.2(12)$ \\
\hline $\mathrm{C}(72)-\mathrm{C}(70)-\mathrm{S}(8)$ & $111.5(11)$ \\
\hline$C(20)-C(21)-C(22)$ & $108.9(10)$ \\
\hline$C(24)-C(21)-C(20)$ & $114.8(11)$ \\
\hline$C(24)-C(21)-C(22)$ & $112.8(11)$ \\
\hline$C(49)-C(48)-C(47)$ & $114.0(12)$ \\
\hline $\mathrm{C}(29)-\mathrm{C}(32)-\mathrm{S}(6)$ & $111.9(9)$ \\
\hline $\mathrm{C}(52)-\mathrm{C}(50)-\mathrm{C}(47)$ & $124.0(14)$ \\
\hline$C(52)-C(50)-C(51)$ & $119.7(18)$ \\
\hline $\mathrm{C}(51)-\mathrm{C}(50)-\mathrm{C}(47)$ & $116.3(18)$ \\
\hline $\mathrm{N}(2)-\mathrm{C}(2)-\mathrm{C}(1)$ & $114.6(16)$ \\
\hline $\mathrm{O}(7)-\mathrm{C}(2)-\mathrm{N}(2)$ & $121.5(14)$ \\
\hline $\mathrm{O}(7)-\mathrm{C}(2)-\mathrm{C}(1)$ & $123.8(13)$ \\
\hline$C(74)-C(73)-C(72)$ & $113.8(14)$ \\
\hline C(45)-C(44)-S(4) & $102.4(10)$ \\
\hline$C(45)-C(44)-C(43)$ & $117.4(11)$ \\
\hline$C(45)-C(44)-C(49)$ & $104.9(9)$ \\
\hline C(43)-C(44)-S(4) & $108.4(8)$ \\
\hline$C(43)-C(44)-C(49)$ & $113.1(12)$ \\
\hline C(49)-C(44)-S(4) & $109.9(9)$ \\
\hline $\mathrm{C}(55)-\mathrm{C}(58)-\mathrm{S}(9)$ & $114.4(10)$ \\
\hline $\mathrm{C}(48)-\mathrm{C}(49)-\mathrm{C}(44)$ & $108.7(11)$ \\
\hline$C(74)-C(75)-C(69)$ & $107.4(13)$ \\
\hline$C(21)-C(24)-C(25)$ & $115.3(13)$ \\
\hline$C(26)-C(24)-C(21)$ & $126.7(13)$ \\
\hline$C(26)-C(24)-C(25)$ & $118.0(14)$ \\
\hline $\mathrm{N}(4)-\mathrm{C}(28)-\mathrm{C}(27)$ & $117(2)$ \\
\hline $\mathrm{O}(18)-\mathrm{C}(28)-\mathrm{N}(4)$ & $122.6(19)$ \\
\hline $\mathrm{O}(18)-\mathrm{C}(28)-\mathrm{C}(27)$ & $120.5(18)$ \\
\hline $\mathrm{C}(70)-\mathrm{C}(72)-\mathrm{C}(73)$ & $108.8(13)$ \\
\hline $\mathrm{C}(73)-\mathrm{C}(74)-\mathrm{C}(75)$ & $111.2(14)$ \\
\hline $\mathrm{C}(73)-\mathrm{C}(74)-\mathrm{C}(76)$ & $114.2(15)$ \\
\hline$C(75)-C(74)-C(76)$ & $114.6(16)$ \\
\hline $\mathrm{C}(77)-\mathrm{C}(76)-\mathrm{C}(74)$ & $117.0(18)$ \\
\hline $\mathrm{C}(78)-\mathrm{C}(76)-\mathrm{C}(74)$ & $125.4(18)$ \\
\hline $\mathrm{C}(78)-\mathrm{C}(76)-\mathrm{C}(77)$ & $117.2(18)$ \\
\hline
\end{tabular}


Symmetry transformations used to generate equivalent atoms: 
Table crystal-4-4. Anisotropic displacement parameters $\left(\AA^{2} \times 10^{3}\right)$ for baran757_0m_a_sq. The anisotropic displacement factor exponent takes the form: $-2 \pi^{2}\left[h^{2} a^{* 2} U^{11}+\ldots+2 h k a^{*} b^{*} U^{12}\right]$

\begin{tabular}{|c|c|c|c|c|c|c|}
\hline & $\mathrm{U}^{11}$ & $\mathrm{U}^{22}$ & $\mathrm{U}^{33}$ & $\mathrm{U}^{23}$ & $\mathrm{U}^{13}$ & $\mathrm{U}^{12}$ \\
\hline $\mathrm{P}(1)$ & $39(2)$ & $41(1)$ & $40(2)$ & $-1(1)$ & $1(2)$ & $-1(1)$ \\
\hline $\mathrm{S}(2)$ & $38(2)$ & $54(1)$ & $44(2)$ & $-3(1)$ & $4(2)$ & $-6(1)$ \\
\hline $\mathrm{P}(2)$ & $38(2)$ & $39(1)$ & $52(2)$ & $4(2)$ & $-4(2)$ & $0(1)$ \\
\hline $\mathrm{S}(3)$ & $59(2)$ & $43(1)$ & $45(2)$ & $3(1)$ & $3(2)$ & $-1(1)$ \\
\hline $\mathrm{S}(4)$ & $37(2)$ & $44(1)$ & $60(2)$ & $10(1)$ & $0(2)$ & $2(1)$ \\
\hline $\mathrm{S}(1)$ & $42(2)$ & $47(1)$ & $57(2)$ & $-3(1)$ & $6(2)$ & $3(1)$ \\
\hline $\mathrm{S}(9)$ & $68(2)$ & $59(2)$ & $46(2)$ & $1(2)$ & $5(2)$ & $4(2)$ \\
\hline $\mathrm{S}(5)$ & $42(2)$ & $48(1)$ & $62(2)$ & $3(2)$ & $1(2)$ & $-6(1)$ \\
\hline$S(6)$ & $49(2)$ & $48(2)$ & $68(3)$ & $7(2)$ & $-6(2)$ & $2(1)$ \\
\hline $\mathrm{S}(8)$ & $56(2)$ & $77(2)$ & $57(3)$ & $-15(2)$ & $-1(2)$ & $-7(2)$ \\
\hline $\mathrm{P}(3)$ & $49(2)$ & $67(2)$ & $47(2)$ & $-16(2)$ & $-3(2)$ & $-4(2)$ \\
\hline$S(7)$ & $61(2)$ & $83(2)$ & $77(3)$ & $-34(2)$ & $-7(2)$ & $6(2)$ \\
\hline $\mathrm{O}(23)$ & $59(5)$ & $38(4)$ & $38(6)$ & $5(4)$ & $-2(5)$ & $-2(3)$ \\
\hline $\mathrm{O}(2)$ & $29(4)$ & $45(4)$ & $42(5)$ & $11(4)$ & $-12(4)$ & $-7(3)$ \\
\hline $\mathrm{O}(12)$ & $43(5)$ & $41(4)$ & $61(6)$ & $-3(4)$ & $9(5)$ & $-3(4)$ \\
\hline $\mathrm{O}(10)$ & $34(4)$ & $49(4)$ & $61(6)$ & 11(4) & $0(5)$ & $-1(3)$ \\
\hline $\mathrm{O}(14)$ & $66(6)$ & $42(4)$ & $45(6)$ & $6(4)$ & $15(5)$ & $0(3)$ \\
\hline $\mathrm{O}(1)$ & $45(5)$ & $60(4)$ & $36(5)$ & $3(4)$ & $8(5)$ & $4(4)$ \\
\hline $\mathrm{O}(3)$ & $44(5)$ & $49(4)$ & $52(6)$ & $-2(4)$ & $0(5)$ & $2(4)$ \\
\hline $\mathrm{O}(11)$ & $39(5)$ & $51(4)$ & $66(7)$ & $7(4)$ & $9(5)$ & $-7(4)$ \\
\hline $\mathrm{O}(24)$ & $63(5)$ & $34(4)$ & $41(5)$ & $1(4)$ & $-1(5)$ & $1(4)$ \\
\hline $\mathrm{O}(5)$ & $73(6)$ & $38(4)$ & $42(6)$ & $-7(4)$ & $-10(5)$ & $0(4)$ \\
\hline $\mathrm{O}(20)$ & $64(6)$ & $52(4)$ & $46(6)$ & $0(4)$ & $-7(5)$ & $7(4)$ \\
\hline $\mathrm{O}(4)$ & $32(4)$ & $35(4)$ & $66(6)$ & $0(4)$ & $5(4)$ & $4(3)$ \\
\hline $\mathrm{O}(6)$ & $62(5)$ & $32(4)$ & $38(6)$ & $5(4)$ & $2(5)$ & $6(3)$ \\
\hline $\mathrm{O}(9)$ & $59(7)$ & $54(4)$ & $49(7)$ & $14(4)$ & $-6(5)$ & $3(4)$ \\
\hline $\mathrm{O}(8)$ & $66(6)$ & $55(4)$ & $52(7)$ & $19(5)$ & $-7(6)$ & $-1(4)$ \\
\hline $\mathrm{O}(21)$ & $75(6)$ & $43(5)$ & $91(8)$ & $1(5)$ & $-34(6)$ & $-2(4)$ \\
\hline $\mathrm{O}(13)$ & $62(6)$ & $57(4)$ & $56(7)$ & $6(4)$ & $0(5)$ & $-13(5)$ \\
\hline $\mathrm{O}(15)$ & $80(6)$ & $36(4)$ & $49(6)$ & $9(4)$ & $1(5)$ & $16(4)$ \\
\hline $\mathrm{N}(3)$ & $53(6)$ & $35(4)$ & $53(8)$ & $1(5)$ & $19(6)$ & $1(4)$ \\
\hline
\end{tabular}




\begin{tabular}{|c|c|c|c|c|c|c|}
\hline $\mathrm{N}(2)$ & $67(7)$ & $38(5)$ & $57(8)$ & $-6(5)$ & $-5(7)$ & $-7(5)$ \\
\hline $\mathrm{O}(19)$ & $61(6)$ & $93(6)$ & $42(6)$ & $-5(5)$ & $-10(5)$ & $6(5)$ \\
\hline $\mathrm{O}(26)$ & $83(7)$ & $51(4)$ & $54(7)$ & $15(5)$ & $3(6)$ & $-2(4)$ \\
\hline $\mathrm{N}(1)$ & $49(6)$ & $43(5)$ & $35(7)$ & $20(5)$ & $-8(5)$ & $-13(4)$ \\
\hline $\mathrm{O}(7)$ & $89(8)$ & $61(5)$ & $72(8)$ & $-2(5)$ & $-19(7)$ & $18(5)$ \\
\hline $\mathrm{O}(17)$ & $81(6)$ & $44(4)$ & $69(7)$ & $9(5)$ & $7(6)$ & $7(4)$ \\
\hline $\mathrm{O}(22)$ & $39(5)$ & $105(7)$ & $74(8)$ & $-8(6)$ & $0(5)$ & $1(5)$ \\
\hline $\mathrm{O}(25)$ & $68(7)$ & $54(5)$ & $77(8)$ & $20(5)$ & $-11(6)$ & $-3(4)$ \\
\hline$C(62)$ & $44(7)$ & $26(5)$ & $40(8)$ & $7(5)$ & $4(7)$ & $1(4)$ \\
\hline$C(61)$ & $58(8)$ & $35(6)$ & $37(8)$ & $7(6)$ & $1(8)$ & $9(5)$ \\
\hline $\mathrm{O}(16)$ & $77(8)$ & $57(5)$ & $93(9)$ & $21(5)$ & $-3(7)$ & $-17(5)$ \\
\hline$C(41)$ & $55(8)$ & $35(6)$ & $30(8)$ & $6(6)$ & $-3(7)$ & $-10(5)$ \\
\hline $\mathrm{N}(6)$ & $67(7)$ & $43(5)$ & $54(8)$ & $-2(5)$ & $9(7)$ & $-9(5)$ \\
\hline $\mathrm{N}(4)$ & 113(11) & $71(7)$ & $61(10)$ & $-4(7)$ & $14(9)$ & $11(8)$ \\
\hline$C(13)$ & $51(7)$ & $27(5)$ & $34(8)$ & $6(5)$ & $-5(7)$ & $-11(5)$ \\
\hline$C(4)$ & $55(10)$ & $38(6)$ & $45(10)$ & $9(6)$ & $2(8)$ & $-4(6)$ \\
\hline $\mathrm{N}(5)$ & $63(6)$ & $27(4)$ & $58(8)$ & $-16(5)$ & $-23(6)$ & $12(4)$ \\
\hline$C(10)$ & $57(8)$ & $29(5)$ & $32(8)$ & $4(6)$ & $-1(6)$ & $-6(5)$ \\
\hline$C(14)$ & $43(8)$ & $31(6)$ & $31(8)$ & $7(5)$ & $-11(6)$ & $3(5)$ \\
\hline$C(9)$ & $47(7)$ & $40(6)$ & $42(9)$ & $1(6)$ & $-17(7)$ & $6(5)$ \\
\hline $\mathrm{O}(27)$ & $86(9)$ & $61(5)$ & $128(12)$ & $-25(6)$ & $-16(8)$ & $14(5)$ \\
\hline$C(3)$ & $62(8)$ & $30(5)$ & $56(10)$ & $21(6)$ & $10(8)$ & $-4(5)$ \\
\hline$C(8)$ & $60(8)$ & $32(5)$ & $38(8)$ & $12(6)$ & $4(7)$ & $3(5)$ \\
\hline $\mathrm{C}(60)$ & $65(8)$ & $29(5)$ & $44(9)$ & $9(6)$ & $-4(7)$ & $11(5)$ \\
\hline$C(64)$ & $66(8)$ & $37(5)$ & $38(8)$ & $4(6)$ & $4(8)$ & $-11(5)$ \\
\hline C(39) & $55(8)$ & $41(6)$ & $38(8)$ & $14(6)$ & $16(7)$ & $3(6)$ \\
\hline$C(56)$ & $64(10)$ & $43(6)$ & $34(9)$ & $5(6)$ & $-4(8)$ & $-2(6)$ \\
\hline C(59) & $58(8)$ & $34(5)$ & $45(9)$ & $-6(6)$ & $-11(7)$ & $-5(5)$ \\
\hline$C(20)$ & $50(7)$ & $49(6)$ & $43(9)$ & $7(6)$ & $-12(7)$ & $11(6)$ \\
\hline$C(7)$ & $51(7)$ & $36(5)$ & $13(6)$ & $-3(5)$ & $10(6)$ & $-1(5)$ \\
\hline $\mathrm{C}(69)$ & $48(8)$ & $68(7)$ & $37(9)$ & $1(7)$ & $-8(7)$ & $7(6)$ \\
\hline$C(65)$ & $48(7)$ & $37(5)$ & $51(9)$ & $2(6)$ & $-14(7)$ & $2(5)$ \\
\hline$C(54)$ & $72(11)$ & $37(7)$ & $88(13)$ & $8(8)$ & $-23(10)$ & $-10(7)$ \\
\hline$C(36)$ & $61(9)$ & $53(7)$ & $31(9)$ & $0(6)$ & $13(7)$ & $-3(6)$ \\
\hline$C(33)$ & $42(7)$ & $37(5)$ & $59(10)$ & $16(6)$ & $12(7)$ & $-3(5)$ \\
\hline$C(45)$ & $27(6)$ & $52(6)$ & $58(10)$ & $5(6)$ & $3(7)$ & $-14(5)$ \\
\hline
\end{tabular}




\begin{tabular}{|c|c|c|c|c|c|c|}
\hline$C(23)$ & $42(7)$ & $46(6)$ & $46(9)$ & $-2(6)$ & $7(7)$ & $-4(5)$ \\
\hline$C(15)$ & $41(7)$ & $46(6)$ & $58(9)$ & $-3(6)$ & $-5(7)$ & $-10(5)$ \\
\hline$C(68)$ & $55(8)$ & $53(6)$ & $38(9)$ & $-1(6)$ & $-3(7)$ & $10(6)$ \\
\hline$C(19)$ & $47(7)$ & $55(6)$ & $34(8)$ & $-5(6)$ & $10(7)$ & $-1(5)$ \\
\hline$C(12)$ & $72(9)$ & $52(6)$ & $40(9)$ & $6(6)$ & $-18(7)$ & $-4(6)$ \\
\hline$C(43)$ & $53(8)$ & $62(7)$ & $47(10)$ & $-13(7)$ & $2(7)$ & $-1(6)$ \\
\hline$C(66)$ & $68(10)$ & $54(8)$ & $31(9)$ & $16(6)$ & $-11(7)$ & $-7(7)$ \\
\hline$C(40)$ & $42(7)$ & $35(5)$ & $60(10)$ & $7(6)$ & $-8(7)$ & $-5(5)$ \\
\hline$C(34)$ & $41(7)$ & $32(5)$ & $48(9)$ & $7(6)$ & $0(6)$ & $1(5)$ \\
\hline$C(35)$ & $61(9)$ & $52(7)$ & $50(10)$ & $2(7)$ & $10(8)$ & $-15(6)$ \\
\hline$C(18)$ & $22(6)$ & $52(6)$ & $49(9)$ & $-7(6)$ & $5(6)$ & $1(5)$ \\
\hline$C(46)$ & $69(9)$ & $50(6)$ & $44(9)$ & $18(6)$ & $13(8)$ & $10(6)$ \\
\hline$C(22)$ & $66(9)$ & $63(7)$ & $44(10)$ & $-2(7)$ & $8(8)$ & $-8(7)$ \\
\hline $\mathrm{O}(18)$ & $177(16)$ & $83(7)$ & $110(13)$ & $-30(8)$ & $11(12)$ & $-44(9)$ \\
\hline$C(63)$ & $48(8)$ & $47(6)$ & $72(11)$ & $27(7)$ & $12(8)$ & $11(5)$ \\
\hline $\mathrm{C}(47)$ & $68(9)$ & $47(6)$ & $59(10)$ & $24(7)$ & $9(9)$ & $-12(6)$ \\
\hline$C(6)$ & $41(7)$ & $55(7)$ & $43(9)$ & $9(6)$ & $5(6)$ & $-8(5)$ \\
\hline$C(29)$ & $88(11)$ & $42(6)$ & $41(9)$ & $-11(7)$ & $20(8)$ & $0(6)$ \\
\hline$C(11)$ & $88(11)$ & $56(7)$ & $46(10)$ & $4(7)$ & $9(9)$ & $4(6)$ \\
\hline$C(16)$ & $32(7)$ & $37(5)$ & 41(9) & $4(5)$ & $-1(6)$ & $3(5)$ \\
\hline$C(55)$ & $67(9)$ & $45(6)$ & $59(11)$ & $4(7)$ & $-1(8)$ & $8(6)$ \\
\hline$C(30)$ & $79(12)$ & $42(7)$ & $72(12)$ & $-7(7)$ & $-3(10)$ & $1(7)$ \\
\hline$C(70)$ & $31(7)$ & $98(9)$ & $64(11)$ & $-7(8)$ & $-11(8)$ & $-3(7)$ \\
\hline$C(21)$ & $32(7)$ & $68(7)$ & $65(11)$ & $-3(7)$ & $-5(7)$ & $-1(6)$ \\
\hline C(48) & $67(9)$ & $56(7)$ & $63(11)$ & $11(7)$ & $1(9)$ & $-6(7)$ \\
\hline$C(17)$ & $36(7)$ & $55(6)$ & $33(8)$ & $-2(6)$ & $-5(6)$ & $-2(5)$ \\
\hline$C(32)$ & $49(8)$ & $50(6)$ & $80(12)$ & $31(7)$ & $7(8)$ & $5(5)$ \\
\hline $\mathrm{C}(42)$ & $61(8)$ & $43(5)$ & $36(8)$ & $6(5)$ & $9(7)$ & $9(6)$ \\
\hline$C(50)$ & $78(11)$ & $45(7)$ & $85(14)$ & $20(9)$ & $2(10)$ & $12(7)$ \\
\hline$C(2)$ & $92(13)$ & $54(8)$ & $45(10)$ & $9(7)$ & $9(9)$ & $9(8)$ \\
\hline$C(73)$ & $60(9)$ & $117(12)$ & $67(12)$ & $-27(11)$ & $-13(10)$ & $14(9)$ \\
\hline$C(53)$ & $117(13)$ & $55(7)$ & $66(12)$ & $-30(8)$ & $18(11)$ & $-12(8)$ \\
\hline $\mathrm{C}(44)$ & $39(7)$ & $48(6)$ & $63(10)$ & $-1(6)$ & $-6(7)$ & $12(6)$ \\
\hline$C(58)$ & $58(9)$ & $62(7)$ & $60(10)$ & $8(7)$ & $9(8)$ & $3(6)$ \\
\hline C(49) & $42(7)$ & $49(6)$ & $81(12)$ & $-2(7)$ & $14(8)$ & $-2(6)$ \\
\hline$C(57)$ & $95(12)$ & $64(8)$ & $71(13)$ & $24(8)$ & $-9(10)$ & $0(8)$ \\
\hline
\end{tabular}




\begin{tabular}{lcccccc}
$\mathrm{C}(75)$ & $71(11)$ & $101(10)$ & $59(12)$ & $12(9)$ & $1(10)$ & $-16(9)$ \\
$\mathrm{C}(38)$ & $57(8)$ & $59(7)$ & $63(11)$ & $12(7)$ & $2(9)$ & $0(6)$ \\
$\mathrm{C}(24)$ & $55(8)$ & $56(7)$ & $53(10)$ & $17(8)$ & $-9(8)$ & $1(6)$ \\
$\mathrm{C}(31)$ & $93(11)$ & $50(7)$ & $93(14)$ & $41(8)$ & $-4(10)$ & $-19(7)$ \\
$\mathrm{C}(37)$ & $80(10)$ & $44(6)$ & $69(12)$ & $5(7)$ & $18(9)$ & $6(6)$ \\
$\mathrm{C}(28)$ & $126(17)$ & $72(10)$ & $65(14)$ & $-27(10)$ & $-10(12)$ & $-20(11)$ \\
$\mathrm{C}(71)$ & $63(9)$ & $113(11)$ & $39(10)$ & $-31(9)$ & $2(8)$ & $3(8)$ \\
$\mathrm{C}(5)$ & $72(10)$ & $85(9)$ & $49(11)$ & $22(8)$ & $10(9)$ & $-1(7)$ \\
$\mathrm{C}(72)$ & $64(10)$ & $95(10)$ & $41(10)$ & $-25(9)$ & $-1(8)$ & $19(8)$ \\
$\mathrm{C}(67)$ & $98(13)$ & $85(9)$ & $79(14)$ & $26(9)$ & $-39(11)$ & $-32(9)$ \\
$\mathrm{C}(52)$ & $88(11)$ & $50(7)$ & $98(15)$ & $0(8)$ & $-19(13)$ & $-18(8)$ \\
$\mathrm{C}(74)$ & $87(12)$ & $114(12)$ & $62(12)$ & $18(10)$ & $-1(11)$ & $25(11)$ \\
$\mathrm{C}(1)$ & $95(12)$ & $66(8)$ & $69(12)$ & $-5(8)$ & $-2(11)$ & $-9(8)$ \\
$\mathrm{C}(76)$ & $87(13)$ & $68(9)$ & $106(17)$ & $-3(10)$ & $-3(12)$ & $-15(9)$ \\
$\mathrm{C}(25)$ & $74(11)$ & $81(9)$ & $112(16)$ & $14(10)$ & $-15(12)$ & $-6(8)$ \\
$\mathrm{C}(26)$ & $89(11)$ & $53(7)$ & $61(11)$ & $3(7)$ & $-11(10)$ & $1(8)$ \\
$\mathrm{C}(77)$ & $109(16)$ & $144(17)$ & $120(20)$ & $-55(16)$ & $-21(15)$ & $48(13)$ \\
$\mathrm{C}(51)$ & $61(11)$ & $83(11)$ & $260(30)$ & $-37(15)$ & $16(16)$ & $-20(9)$ \\
$\mathrm{C}(78)$ & $109(15)$ & $103(12)$ & $98(17)$ & $-23(12)$ & $-6(14)$ & $-27(11)$ \\
$\mathrm{C}(27)$ & $180(20)$ & $84(10)$ & $77(15)$ & $-19(10)$ & $-3(16)$ & $40(13)$ \\
& & & & & & \\
\hline
\end{tabular}


Table crystal-4-5. Hydrogen coordinates $\left(\mathrm{x} 10^{4}\right)$ and isotropic displacement parameters $\left(\AA^{2} \mathrm{x} 10^{3}\right)$ for baran757_0m_a_sq.

\begin{tabular}{|c|c|c|c|c|}
\hline & $\mathrm{x}$ & $\mathrm{y}$ & $\mathrm{z}$ & $\mathrm{U}(\mathrm{eq})$ \\
\hline $\mathrm{H}(2 \mathrm{~A})$ & 689 & 5406 & 4105 & 57 \\
\hline $\mathrm{H}(2)$ & -7855 & 2680 & 4183 & 65 \\
\hline $\mathrm{H}(1 \mathrm{D})$ & -4073 & 4237 & 3382 & 51 \\
\hline $\mathrm{H}(9)$ & 8243 & 9208 & 2605 & 52 \\
\hline $\mathrm{H}(3)$ & 2008 & 8177 & 2878 & 66 \\
\hline $\mathrm{H}(1)$ & -3053 & 7230 & 4101 & 98 \\
\hline $\mathrm{H}(5)$ & 4980 & 10045 & 2630 & 59 \\
\hline $\mathrm{H}(9 \mathrm{~A})$ & -1271 & 3519 & 3771 & 52 \\
\hline $\mathrm{H}(8)$ & -2840 & 3497 & 4281 & 52 \\
\hline $\mathrm{H}(7)$ & 7063 & 8981 & 3091 & 55 \\
\hline $\mathrm{H}(11 \mathrm{~A})$ & 3304 & 9568 & 1944 & 71 \\
\hline $\mathrm{H}(11 \mathrm{~B})$ & 3235 & 9948 & 2091 & 71 \\
\hline $\mathrm{H}(11 \mathrm{C})$ & 2282 & 9632 & 2286 & 71 \\
\hline $\mathrm{H}(10)$ & 3316 & 9308 & 2935 & 55 \\
\hline $\mathrm{H}(20 \mathrm{~A})$ & 1590 & 4468 & 5621 & 57 \\
\hline $\mathrm{H}(20 \mathrm{~B})$ & 3269 & 4181 & 5547 & 57 \\
\hline $\mathrm{H}(7 \mathrm{~A})$ & -6424 & 3700 & 3963 & 40 \\
\hline $\mathrm{H}(22)$ & 8929 & 9210 & 3908 & 61 \\
\hline $\mathrm{H}(4)$ & -1728 & 6188 & 3838 & 55 \\
\hline $\mathrm{H}(14 \mathrm{~A})$ & 4156 & 6401 & 2851 & 55 \\
\hline $\mathrm{H}(23 \mathrm{~A})$ & -865 & 4032 & 4929 & 54 \\
\hline $\mathrm{H}(15 \mathrm{~A})$ & 504 & 4811 & 3526 & 72 \\
\hline $\mathrm{H}(15 \mathrm{~B})$ & 1420 & 4652 & 3848 & 72 \\
\hline $\mathrm{H}(15 \mathrm{C})$ & -96 & 4974 & 3864 & 72 \\
\hline $\mathrm{H}(15)$ & 4597 & 9794 & 3187 & 58 \\
\hline $\mathrm{H}(19 \mathrm{~A})$ & 3081 & 4586 & 5127 & 55 \\
\hline $\mathrm{H}(19 \mathrm{~B})$ & 2551 & 4207 & 5001 & 55 \\
\hline $\mathrm{H}(12 \mathrm{D})$ & -5998 & 3450 & 2993 & 82 \\
\hline $\mathrm{H}(12 \mathrm{E})$ & -6416 & 3855 & 2972 & 82 \\
\hline $\mathrm{H}(12 \mathrm{~F})$ & -7210 & 3640 & 3274 & 82 \\
\hline
\end{tabular}




\begin{tabular}{|c|c|c|c|c|}
\hline H(1RA) & 5092 & 5654 & 2192 & 81 \\
\hline $\mathrm{H}(1 \mathrm{SA})$ & 3762 & 5979 & 2084 & 81 \\
\hline $\mathrm{H}(1 \mathrm{TA})$ & 2799 & 5693 & 2315 & 81 \\
\hline $\mathrm{H}(1 \mathrm{NA})$ & -504 & 5736 & 3524 & 55 \\
\hline $\mathrm{H}(8 \mathrm{~A})$ & 2010 & 6526 & 3692 & 48 \\
\hline $\mathrm{H}(6 \mathrm{~A})$ & 3321 & 6246 & 4132 & 65 \\
\hline $\mathrm{H}(1 \mathrm{DA})$ & 2216 & 6756 & 2503 & 65 \\
\hline $\mathrm{H}(1 \mathrm{EA})$ & 2520 & 6482 & 2219 & 65 \\
\hline $\mathrm{H}(22 \mathrm{~A})$ & -2700 & 3920 & 5401 & 69 \\
\hline $\mathrm{H}(22 \mathrm{~B})$ & -2239 & 4303 & 5526 & 69 \\
\hline $\mathrm{H}(12 \mathrm{~A})$ & 8444 & 9647 & 2199 & 84 \\
\hline $\mathrm{H}(12 \mathrm{~B})$ & 7205 & 9946 & 2023 & 84 \\
\hline $\mathrm{H}(12 \mathrm{C})$ & 7054 & 9558 & 1896 & 84 \\
\hline $\mathrm{H}(1 \mathrm{OA})$ & 4989 & 6884 & 2133 & 69 \\
\hline $\mathrm{H}(6 \mathrm{~B})$ & -8288 & 3152 & 4591 & 56 \\
\hline $\mathrm{H}(6 \mathrm{C})$ & -8363 & 3261 & 4223 & 56 \\
\hline $\mathrm{H}(11 \mathrm{D})$ & -1111 & 3830 & 3192 & 95 \\
\hline $\mathrm{H}(11 \mathrm{E})$ & -2568 & 3912 & 2895 & 95 \\
\hline $\mathrm{H}(11 \mathrm{~F})$ & -2017 & 3521 & 2982 & 95 \\
\hline $\mathrm{H}(16)$ & -5312 & 4267 & 4040 & 44 \\
\hline $\mathrm{H}(21 \mathrm{~A})$ & 146 & 3966 & 5768 & 66 \\
\hline $\mathrm{H}(13)$ & 6328 & 6359 & 2038 & 74 \\
\hline $\mathrm{H}(19)$ & 8088 & 6568 & 2216 & 74 \\
\hline $\mathrm{H}(17 \mathrm{~B})$ & 187 & 5057 & 5127 & 62 \\
\hline $\mathrm{H}(17 \mathrm{C})$ & -502 & 4833 & 5430 & 62 \\
\hline H(17D) & -2076 & 4907 & 5146 & 62 \\
\hline $\mathrm{H}(6 \mathrm{AA})$ & -3454 & 7006 & 3538 & 72 \\
\hline $\mathrm{H}(6 \mathrm{BA})$ & -3639 & 6716 & 3809 & 72 \\
\hline $\mathrm{H}(1 \mathrm{~A})$ & 4948 & 4968 & 3511 & 70 \\
\hline $\mathrm{H}(1 \mathrm{~B})$ & 5968 & 5132 & 3824 & 70 \\
\hline $\mathrm{H}(1 \mathrm{C})$ & 6185 & 5322 & 3486 & 70 \\
\hline $\mathrm{H}(1 \mathrm{Z})$ & 12927 & 9039 & 4548 & 98 \\
\hline $\mathrm{H}(20)$ & 11298 & 9285 & 4716 & 98 \\
\hline $\mathrm{H}(21)$ & 1458 & 7888 & 2417 & 119 \\
\hline $\mathrm{H}(23)$ & 2963 & 7566 & 2452 & 119 \\
\hline $\mathrm{H}(24)$ & 3382 & 7852 & 2185 & 119 \\
\hline
\end{tabular}




\begin{tabular}{|c|c|c|c|c|}
\hline $\mathrm{H}(25)$ & 1626 & 8542 & 3316 & 72 \\
\hline $\mathrm{H}(26)$ & 1409 & 8805 & 3025 & 72 \\
\hline $\mathrm{H}(1 \mathrm{G})$ & 7400 & 6271 & 2699 & 69 \\
\hline $\mathrm{H}(1 \mathrm{H})$ & 7865 & 5997 & 2421 & 69 \\
\hline $\mathrm{H}(5 \mathrm{~A})$ & 6368 & 7754 & 3531 & 115 \\
\hline $\mathrm{H}(5 \mathrm{~B})$ & 4491 & 7780 & 3773 & 115 \\
\hline $\mathrm{H}(5 \mathrm{C})$ & 6264 & 8062 & 3784 & 115 \\
\hline $\mathrm{H}(29)$ & 7486 & 9213 & 4560 & 92 \\
\hline $\mathrm{H}(1 \mathrm{~K})$ & 6964 & 8916 & 4305 & 92 \\
\hline $\mathrm{H}(1 \mathrm{~L})$ & -1524 & 5871 & 4786 & 89 \\
\hline $\mathrm{H}(17 \mathrm{~A})$ & -1552 & 5483 & 4658 & 89 \\
\hline $\mathrm{H}(1 \mathrm{M})$ & -2619 & 5781 & 4454 & 89 \\
\hline $\mathrm{H}(2 \mathrm{OA})$ & -476 & 7753 & 3075 & 118 \\
\hline $\mathrm{H}(2 \mathrm{PA})$ & 1366 & 7484 & 3076 & 118 \\
\hline $\mathrm{H}(2 \mathrm{NA})$ & 1319 & 7791 & 3331 & 118 \\
\hline $\mathrm{H}(1 \mathrm{FA})$ & 3457 & 5682 & 4484 & 96 \\
\hline $\mathrm{H}(1 \mathrm{IA})$ & 2004 & 5432 & 4685 & 96 \\
\hline $\mathrm{H}(1 \mathrm{JA})$ & 2377 & 5819 & 4803 & 96 \\
\hline $\mathrm{H}(2 \mathrm{~K})$ & 10285 & 10032 & 4470 & 108 \\
\hline $\mathrm{H}(2 \mathrm{~L})$ & 9289 & 9710 & 4652 & 108 \\
\hline $\mathrm{H}(2 \mathrm{M})$ & 7954 & 9934 & 4409 & 108 \\
\hline $\mathrm{H}(5 \mathrm{D})$ & -3291 & 2570 & 5108 & 103 \\
\hline $\mathrm{H}(5 \mathrm{E})$ & -5279 & 2651 & 5315 & 103 \\
\hline $\mathrm{H}(5 \mathrm{~F})$ & -3690 & 2952 & 5238 & 103 \\
\hline $\mathrm{H}(2 \mathrm{Q})$ & 12963 & 9606 & 4312 & 80 \\
\hline $\mathrm{H}(2 \mathrm{R})$ & 12426 & 9320 & 4048 & 80 \\
\hline $\mathrm{H}(2 \mathrm{~S})$ & 11414 & 10318 & 2826 & 131 \\
\hline $\mathrm{H}(2 \mathrm{~T})$ & 11471 & 10174 & 3184 & 131 \\
\hline $\mathrm{H}(2 \mathrm{U})$ & 10371 & 10533 & 3108 & 131 \\
\hline $\mathrm{H}(32 \mathrm{~A})$ & 5558 & 7239 & 2990 & 94 \\
\hline $\mathrm{H}(31 \mathrm{~A})$ & 3713 & 6975 & 2887 & 94 \\
\hline $\mathrm{H}(2 \mathrm{X})$ & 9777 & 8786 & 4653 & 106 \\
\hline $\mathrm{H}(1 \mathrm{E})$ & -8129 & 2160 & 4041 & 115 \\
\hline $\mathrm{H}(1 \mathrm{~F})$ & -6687 & 1898 & 4230 & 115 \\
\hline $\mathrm{H}(1 \mathrm{I})$ & -6108 & 2017 & 3875 & 115 \\
\hline $\mathrm{H}(25 \mathrm{~A})$ & 4069 & 3566 & 5372 & 134 \\
\hline
\end{tabular}




\begin{tabular}{lrrrr}
$\mathrm{H}(25 \mathrm{~B})$ & 2964 & 3254 & 5552 & 134 \\
$\mathrm{H}(25 \mathrm{C})$ & 3332 & 3611 & 5735 & 134 \\
$\mathrm{H}(26 \mathrm{~A})$ & -1371 & 3514 & 5145 & 81 \\
$\mathrm{H}(26 \mathrm{~B})$ & 443 & 3223 & 5160 & 81 \\
$\mathrm{H}(33)$ & 13573 & 8604 & 4327 & 188 \\
$\mathrm{H}(34)$ & 12889 & 8307 & 4084 & 188 \\
$\mathrm{H}(35)$ & 12274 & 8283 & 4454 & 188 \\
$\mathrm{H}(1 \mathrm{P})$ & 8960 & 6971 & 2455 & 200 \\
$\mathrm{H}(1 \mathrm{Q})$ & 8490 & 7263 & 2714 & 200 \\
$\mathrm{H}(1 \mathrm{U})$ & 7857 & 7320 & 2349 & 200 \\
$\mathrm{H}(39)$ & 9918 & 8320 & 3843 & 124 \\
$\mathrm{H}(3 \mathrm{~A})$ & 8261 & 8628 & 3912 & 124 \\
$\mathrm{H}(2 \mathrm{ZA})$ & -1836 & 7950 & 4328 & 169 \\
$\mathrm{H}(30 \mathrm{~A})$ & -1127 & 7712 & 4620 & 169 \\
$\mathrm{H}(2 \mathrm{YA})$ & -3216 & 7632 & 4438 & 169 \\
& & & & \\
\hline
\end{tabular}




\section{IX) Abbreviations}

MAC reagent $=2-($ tert-butyldimethylsilanyloxy $)$ malononitrile;

$\mathrm{rt}=$ room temperature;

TFE = 2,2,2-trifluoroethanol;

TBAF = tetra- $n$-butylammonium fluoride;

DMS = dimethyl sulfide;

PCNBCl = $p$-cyanobenzoyl chloride;

DMAP = 4-dimethylaminopyridine;

TCDI = 1,1'-thiocarbonyldiimidazole;

$\mathrm{BHT}=$ dibutylhydroxytoluene;

PTSA $=p$-toluenesulfonic acid;

$\mathrm{NMO}=N$-methylmorpholine $\mathrm{N}$-oxide;

$\mathrm{DBDMH}=$ 1,3-dibromo-5,5-dimethylhydantoin;

$\mathrm{AcOH}=$ acetic acid;

$\mathrm{TfOH}=$ trifluoromethanesulfonic acid;

AIBN = 2,2'-azobis(2-methylpropionitrile);

PPTS = pyridinium $p$-toluenesulfonate;

DBN = 1,5-diazabicyclo(4.3.0)non-5-ene;

TMSOK $=$ potassium trimethylsilanolate. 


\section{X) References}

[1] Donohoe, T. J.; Garg, R.; Moore, P. R. Tetrahedron Lett. 1996, 37, 3407.

[2] Olah, G. A.; Westrman, P. W.; Melby, E. G.; Mo, Y. K. J. Am. Chem. Soc. 1974, 96, 3565.

[3] Aliev, A. E.; Karu, K.; Mitchell, R. E.; Porter, M. J. Org. Biomol. Chem., 2016, 14, 238.

[4] Morales, S.; Guijarro, F. G.; Ruano, J. L. G.; Cid, M. B. J. Am. Chem. Soc. 2014, 136, 1082.

[5] Yang, K. S.; Nibbs, A. E.; Turkmen, Y. E.; Rawal, V. H. J. Am. Chem. Soc. 2013, 135, 16050.

[6] Richards, K. D.; Kolar, A. J.; Srinivasan, A.; Stephenson, R. W.; Olsen, R. K. J. Org.

Chem. 1976, 41, 3674.

[7] Siricilla, S.; Mitachi, K.; Wan, B.; Franzblau, S. G.; Kurosu, M. The Journal of

Antibiotics, 2015, 68, 271. 